\title{
The Rydberg Constant and Proton Size from Atomic Hydrogen
}

\author{
Axel Beyer
}
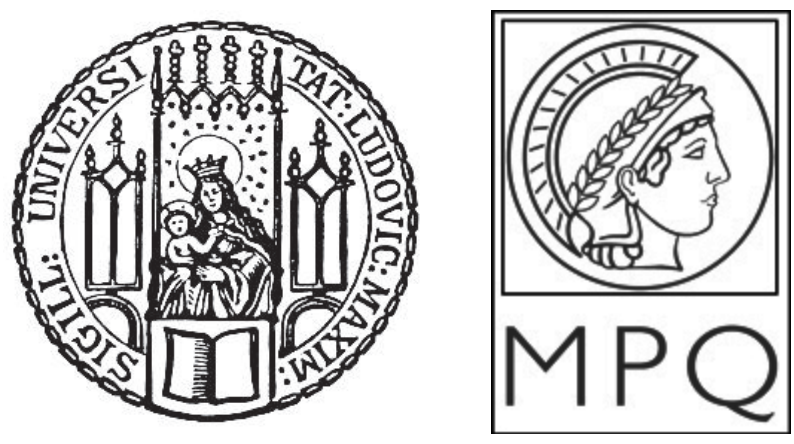

Munich 2016 



\title{
The Rydberg Constant and Proton Size from Atomic Hydrogen
}

\author{
Axel Beyer
}

\author{
Dissertation \\ performed in the Laser Spectroscopy Division \\ of the Max Planck Institute of Quantum Optics \\ Garching \\ presented to the Faculty of Physics \\ of the Ludwig Maximilian University \\ Munich \\ by \\ Axel Beyer \\ from Sonneberg
}

Munich, March 23rd, 2016 
Erstgutachter: Prof. Dr. Theodor W. Hänsch Zweitgutachter: Prof. Dr. Joachim Rädler Tag der mündlichen Prüfung: 18. Mai 2016 


\section{Zusammenfassung}

Das Wasserstoffatom (H) stellt ein einzigartiges System für Tests der Quanten-Elektrodynamik dar. Aufgrund seiner einfachen Struktur und genauen theoretischen Beschreibung liefert es außerdem wichtige Daten für die Bestimmung der RydbergKonstante $R_{\infty}$ und des Proton-Ladungsradius $r_{\mathrm{p}}$ im Rahmen der globalen Anpassung fundamentaler Konstanten durch das Committee on Data for Science and Technology (CODATA). Im Jahre 2010 kam das sogenannte "proton size puzzle" auf, eine Diskrepanz von sieben Standardabweichungen zwischen CODATA und dem zehn mal genauer gemessenen Wert von $r_{\mathrm{p}}$ in myonischem Wasserstoff $(\mu-\mathrm{p},[1,2])$. Vorschläge zur Lösung dieses Rätsels reichen bis hin zu Physik, die über das Standard-Modell hinaus geht [3].

Die vorliegende Arbeit stellt eine neue Technik zur hochauflösenden Vermessung von $2 \mathrm{~S}-n P$ Dipol-Übergängen in $\mathrm{H}$ vor, die einen kryogenen Strahl von H-Atomen verwendet und durch selektive optische Anregung in den $2 \mathrm{~S}_{1 / 2}^{F=0}$ Zustand bringt. Solche Messungen können für eine neue Bestimmung von $R_{\infty}$ und $r_{\mathrm{p}}$ verwendet werden und bringen neue Erkenntnisse im Rätsel um $r_{\mathrm{p}}$. Die Technik wurde zunächst zur Bestimmung der 2S-4P Übergangsfrequenz $\nu_{2 S-4 P}$ und der Feinstruktur-Aufspaltung (FS) zwischen dem $4 \mathrm{P}_{1 / 2}^{F=1}$ und $4 \mathrm{P}_{3 / 2}^{F=1}$ Zustand verwendet:

$$
\begin{aligned}
\nu_{2 S-4 P} & =616520931625.8(2.2) \mathrm{kHz} \\
\Delta \nu_{F S}^{\exp }(4 \mathrm{P}) & =1367432.7(2.8) \mathrm{kHz} .
\end{aligned}
$$

Letztere stellt die genaueste Messung einer FS-Aufspaltung in $\mathrm{H}$ dar und stimmt mit dem nochmals genaueren theoretischen Wert $\Delta \nu_{F S}^{\text {theo }}(4 \mathrm{P})=1367433.3(3) \mathrm{kHz}$ überein [4].

Mit einer Unsicherheit von $2.2 \mathrm{kHz}$ ist $\nu_{2 S-4 P}$ um $6.8 \mathrm{bzw} .4 .5$ mal genauer bestimmt als in den genauesten vorherigen Messungen der $2 \mathrm{~S}_{-} 4 \mathrm{P}_{1 / 2}$ und $2 \mathrm{~S}-4 \mathrm{P}_{3 / 2}$ Übergangsfrequenzen [5]. Unter Benutzung der QED Ausdrücke für die Energieniveaus in $\mathrm{H}$ werden aus $\nu_{2 S-4 P}$ und der 1 S-2S Übergangsfrequenz $[6,7] R_{\infty}$ und $r_{\mathrm{p}}$ bestimmt:

$$
\begin{aligned}
R_{\infty} & =10973731.568037(91) \mathrm{m}^{-1} \\
r_{\mathrm{p}} & =0.8293(91) \mathrm{fm} .
\end{aligned}
$$

Diese Werte sind ebenso genau, wie diejenigen, die aus dem gesamten Datensatz (15 Messungen) aller vorheriger Präzisionsmessungen an $\mathrm{H}$ in CODATA bestimmt werden. Während eine Diskrepanz von 3.8 Standardabweichungen zu letzteren besteht, stimmen die Ergebnisse dieser Arbeit und mit denen aus $\mu-p$ überein.

Das 2S-4P Experiment ist in erster Linie unbeeinflusst von den systematischen Effekten, die die Unsicherheiten der genauesten vorherigen Bestimmungen von $R_{\infty}$ aus der Spektroskopie von Zwei-Photonen-Übergängen in H dominieren. Stattdessen wird die Unsicherheit hauptsächlich durch die Dopplerverschiebung, die durch Verwendung eines aktiven, faser-basierten Retroreflektors klein gehalten wird, und durch Linien-Asymmetrien aufgrund von Quanteninterferenz (QI) von benachbarten Zuständen bestimmt. Der QI-Effekt erlangte erst vor Kurzem Aufmerksamkeit in der Präzisionsspektroskopie [8, 9]. Scheinbare QI-Linienverschiebungen wurden experimentell studiert und stellen die erste direkte Beobachtung dieses Effektes in der Präzisionsspektroskopie weit von einander entfernter Resonanzen dar. Die beobachteten Linienverschiebungen von $\pm 51 \mathrm{kHz}$ sind sechs mal größer als das proton size puzzle gemessen am 2S-4P Übergang. Diese werden durch ein geeignetes LinienformModell unter Kontrolle gebracht, das im Rahmen der theoretischen und experimentellen Studien dieser Arbeit hergeleitet und validiert wurde. 



\section{Abstract}

The hydrogen atom $(\mathrm{H})$ is a unique system for tests of quantum electrodynamics (QED). Due to its simplicity and accurate theoretical description, it also provides key input data for the determination of the Rydberg constant $R_{\infty}$ and the proton root mean square (r.m.s.) charge radius $r_{\mathrm{p}}$ in the global adjustment of fundamental constants [4] by the Committee on Data for Science and Technology (CODATA). In the year 2010, the "proton size puzzle" emerged, which refers to a discrepancy of seven standard deviations between CODATA and a ten times more accurate measurement of $r_{\mathrm{p}}$ in muonic hydrogen $(\mu-\mathrm{p},[1,2])$. Proposed solutions for this puzzle cover a wide range of scenarios, up to physics beyond the standard model [3].

This thesis reports on a novel scheme for high resolution spectroscopy of dipole allowed $2 \mathrm{~S}-n \mathrm{P}$ transitions in $\mathrm{H}$, using a cryogenic beam of $\mathrm{H}$ atoms that are prepared in the meta-stable $2 \mathrm{~S}_{1 / 2}^{F=0}$ state by state selective optical excitation. Such measurements can be used for a new determination of $R_{\infty}$ and $r_{\mathrm{p}}$ from $\mathrm{H}$ spectroscopy, shedding new light on the "proton size puzzle". The scheme has been applied to spectroscopy of the $2 \mathrm{~S}-4 \mathrm{P}$ transition first, yielding:

$$
\begin{aligned}
\nu_{2 S-4 P} & =616520931625.8(2.2) \mathrm{kHz} \\
\Delta \nu_{F S}^{\exp }(4 \mathrm{P}) & =1367432.7(2.8) \mathrm{kHz}
\end{aligned}
$$

for the $2 \mathrm{~S}-4 \mathrm{P}$ absolute transition frequency $\nu_{2 S-4 P}$ and the fine structure splitting $\Delta \nu_{F S}^{\exp }(4 \mathrm{P})$ of the $4 \mathrm{P}_{1 / 2}^{F=1}$ and $4 \mathrm{P}_{3 / 2}^{F=1}$ states. The latter represents the most accurately measured fine structure splitting in $\mathrm{H}$ and is found to be in agreement with the even more accurate theoretical value of $\Delta \nu_{F S}^{\text {theo }}(4 \mathrm{P})=1367433.3(3) \mathrm{kHz}[4]$.

The $2.2 \mathrm{kHz}$ uncertainty of $\nu_{2 S-4 P}$ corresponds to improvements in accuracy by factors of 6.8 and 4.5 compared to previous best measurements of the $2 \mathrm{~S}_{-}-4 \mathrm{P}_{1 / 2}$ and $2 \mathrm{~S}-4 \mathrm{P}_{3 / 2}$ transitions [5]. Utilizing QED expressions for the energy levels in $\mathrm{H}$ [4], $\nu_{2 S-4 P}$ is used in combination with the $1 \mathrm{~S}-2 \mathrm{~S}$ transition frequency $[6,7]$, to extract values of $R_{\infty}$ and $r_{\mathrm{p}}$ :

$$
\begin{aligned}
R_{\infty} & =10973731.568037(91) \mathrm{m}^{-1} \\
r_{\mathrm{p}} & =0.8293(91) \mathrm{fm} .
\end{aligned}
$$

These values are as accurate as the ones determined from the aggregate world data of precision H spectroscopy (15 measurements) that enter the CODATA adjustment. While a discrepancy of 3.8 combined standard deviations is found to the latter, the presented results agree with the measurements in $\mu-\mathrm{p}$.

The 2S-4P experiment is essentially unaffected by the systematic effects dominating the uncertainties in the previous most precise determinations of $R_{\infty}$ using dipole forbidden two photon transitions in $H$. Instead, the main systematic effects are the first order Doppler effect, canceled by the use of an active fiber-based retroreflector (AFR) developed in this thesis, and line shape distortions due to quantum interference (QI) of neighboring atomic resonances. The latter effect has come to the attention of the precision spectroscopy community only recently [8, 9]. Apparent QI line shifts have been studied experimentally, yielding the first direct observation in precision spectroscopy of largely separated atomic resonances. The observed shifts of up to $\pm 51 \mathrm{kHz}$ are six times larger than the proton size discrepancy for the $2 \mathrm{~S}-4 \mathrm{P}$ transition. They are brought under control by a suitable line shape model function, derived and validated within the theoretical and experimental studies of this work. 



\section{Contents}

List of Figures $\quad$ v

$\begin{array}{ll}\text { 1. Introduction } & \mathbf{1}\end{array}$

1.1. The dawn of modern physics . . . . . . . . . . . . . . . . 1

1.2. QED description of the hydrogen atom . . . . . . . . . . . . . 2

1.3. Determination of external input parameters . . . . . . . . . . . 3

1.4. The proton size puzzle . . . . . . . . . . . . . . 4

1.5. Improving the hydrogen input data . . . . . . . . . . . . . . 6

1.6. Advantages of the Garching 2S-4P apparatus . . . . . . . . . . . . . 7

2. Theory of quantum interference from neighboring atomic resonances $\mathbf{1 1}$

2.1. Classical analog: Two driven antennas . . . . . . . . . . . . . . . . 12

2.2. Atomic line shape models . . . . . . . . . . . . . . . . . . . . . 14

2.2.1. Expanded line shape . . . . . . . . . . . . . . . . . . . . 15

2.2.2. Perturbative approach . . . . . . . . . . . . . 16

2.2.3. Master-equation approach . . . . . . . . . . . . . 18

2.3. Application to hydrogen $2 \mathrm{~S}-4 \mathrm{P}$. . . . . . . . . . . . . . . . . . . . . . 19

2.3.1. Relevant energy levels . . . . . . . . . . . . . . . . . 19

2.3.2. Expanded line shape . . . . . . . . . . . . . . . . 21

2.3.3. Comparison of line shape models in the limit of small excita-

tion rates . . . . . . . . . . . . . . . . . . 22

2.4. Properties of QI line shifts . . . . . . . . . . . . . . . . . 23

2.4.1. Spherical components . . . . . . . . . . . . . 25

2.4.2. Finite detection angle, symmetry and periodicity . . . . . 25

2.4.3. Cancellations in special arrangements . . . . . . . . . . . . 27

3. Experimental setup $\mathbf{3 5}$

3.1. Cryogenic beam of hydrogen $2 \mathrm{~S}$ atoms . . . . . . . . . . . . 35

3.2. XUV fluorescence detection . . . . . . . . . . . . . . 37

3.3. Laser spectroscopy setup . . . . . . . . . . . . . . . . . . . . . . . 39

3.4. Laser frequency calibration . . . . . . . . . . . . . . . . . . . . . . . . . . . . . . . . 40

3.5. Suppression of the first order Doppler effect . . . . . . . . . . . . . . 40

3.5.1. Principle of two beam excitation . . . . . . . . . . . . . 41

3.5.2. Corner cubes and optical cavities . . . . . . . . . . . . . . . 42

3.5.3. Active fiber-based retroreflector concept . . . . . . . . . . . 44 
4. Measurement of the residual uncompensated Doppler shift

5. Line distortions due to the coherent interaction with two laser beams 51

6. Measurement of quantum interference line shifts $\mathbf{5 5}$

6.1. Experimental data . . . . . . . . . . . . . . . . . . 55

6.1.1. Data acquisition . . . . . . . . . . . . . 55

6.1.2. Raw data analysis . . . . . . . . . . . . . . . 55

6.1.3. Observed QI line shifts . . . . . . . . . . . . . . 57

6.2. Simulation of experimental line shapes . . . . . . . . . . . . . 58

7. Measurement of the 2S-4P absolute transition frequency 63

7.1. Data evaluation . . . . . . . . . . . . . . . . . 63

7.2. 2S-4P absolute transition frequency . . . . . . . . . . 63

7.3. 4P fine structure interval . . . . . . . . . . . . . . . . 65

7.4. Systematic effects . . . . . . . . . . . . . . . . 65

7.4.1. First order Doppler shift . . . . . . . . . . . . . 65

7.4.2. Radiation force induced line distortions . . . . . . . . . . . 65

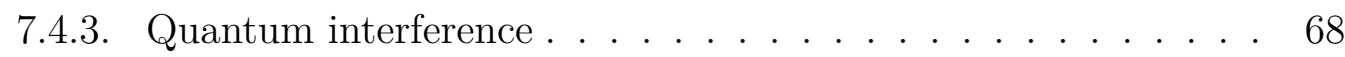

7.4.4. Second order Doppler effect . . . . . . . . . . . . . 69

7.4.5. DC Stark effect . . . . . . . . . . . . . . . . 70

7.4.6. Laser intensity dependence . . . . . . . . . . . . . . . . . . . 71

7.4.7. Laser spectrum and frequency . . . . . . . . . . . . . . 72

7.4.8. Recoil shift . . . . . . . . . . . . . . . . 72

7.4.9. Zeeman Effect . . . . . . . . . . . . . 72

7.4.10. Hyperfine corrections . . . . . . . . . . . . . . 73

8. Rydberg constant and proton charge radius $\quad \mathbf{7 5}$

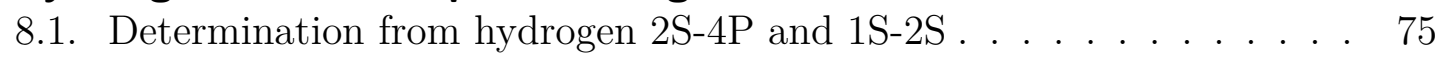

8.2. Conclusions and Outlook . . . . . . . . . . . . . . 75

8.2.1. Hydrogen 2S-4P . . . . . . . . . . . . . . . . 75

8.2.2. Future prospects . . . . . . . . . . . . . . 78

$\begin{array}{ll}\text { Appendix } & 83\end{array}$

A. Data analysis: Tests of internal consistency $\mathbf{8 3}$

A.1. Re-evaluation of 2013 measurement run . . . . . . . . . . . . . . . . . 83

A.2. Center of weight method for 2014 data . . . . . . . . . . . . . 85

A.3. Correcting Voigt fits using master-equation approach (2014 data) . . 86

B. Systematic uncertainties in master-equation approach $\quad 87$

B.1. Experimental restrictions . . . . . . . . . . . . . . . . . . . 87

B.1.1. Observed line centers . . . . . . . . . . . . . . . . . 87

B.1.2. Observed line strengths . . . . . . . . . . . . . 87 
B.1.3. Linear laser polarization angle . . . . . . . . . . . . . . . . . . 89

B.2. Detector acceptance and efficiency . . . . . . . . . . . . . . . . 90

B.2.1. Imperfections in detector geometry . . . . . . . . . . . . . . 90

B.2.2. Simulation of electric field configuration . . . . . . . . . . . 91

B.2.3. Emission direction dependent quantum efficiency . . . . . . . 92

B.2.4. Emission polarization dependent quantum efficiency . . . . . . 93

B.3. Atomic beam parameters and saturation . . . . . . . . . . . . . 94

C. Active fiber-based retroreflector 95

C.1. Minimization of imperfections . . . . . . . . . . . . . . . . . . . . 95

C.2. Active Stabilization . . . . . . . . . . . . . . . . . . . 101

D. Theory of quantum interference from neighboring atomic transitions $\mathbf{1 0 3}$

D.1. Dipole matrix elements for hydrogen . . . . . . . . . . . . . . . . 103

D.2. Arbitrary laser polarization . . . . . . . . . . . . . . . . . . 104

D.3. Emitted Intensity Pattern . . . . . . . . . . . . . . . . . . 105

D.3.1. Interference of spherical components . . . . . . . . . . . 106

D.3.2. Line shape distortions . . . . . . . . . . . . . . . . . 107

D.3.3. Finite detection angle . . . . . . . . . . . . . . . . . . . 108

D.4. Expanded Line Shape . . . . . . . . . . . . . . . . . . . . . 108

D.4.1. General treatment . . . . . . . . . . . . . . . 108

D.4.2. Application to hydrogen 2S-4P . . . . . . . . . . . . . . 109

D.4.3. Finite detection angle . . . . . . . . . . . . . . . . 110

D.4.4. General Detection Geometry . . . . . . . . . . . . 111

E. Hydrogen apparatus: CAD drawings $\mathbf{1 1 3}$

E.1. 2014 measurement campaign . . . . . . . . . . . . . . . . . . . . . . . 113

E.2. 2013 measurement campaign . . . . . . . . . . . . . . . . . . . . . 113

$\begin{array}{ll}\text { Bibliography } & 117\end{array}$

$\begin{array}{ll}\text { Acknowledgments } & 125\end{array}$

$\begin{array}{ll}\text { List of publications and presentations } & 127\end{array}$ 



\section{List of Figures}

1.1. Visible spectrum of hydrogen . . . . . . . . . . . . . . . 2

1.2. Energy levels in atomic hydrogen . . . . . . . . . . . . . . 3

1.3. Determinations of the Rydberg constant and proton charge radius . . 5

2.1. Classical analog to apparent line shifts due to quantum interference from neighboring resonances . . . . . . . . . . . . . . . . 13

2.2. Level scheme for quantum interference from neighboring resonances . 14

2.3. Coordinate system used throughout this text . . . . . . . . . . . 17

2.4. Relevant energy levels for hydrogen 2S-4P spectroscopy . . . . . . . . 21

2.5. Line shapes of the $2 \mathrm{~S}_{1 / 2}^{F=0}$ to $4 \mathrm{P}_{1 / 2}^{F=1}$ and $4 \mathrm{P}_{3 / 2}^{F=1}$ transitions . . . . . 24

2.6. Perturbative model: QI line shifts $\Delta \nu_{\mathrm{QI}}$ for the $2 \mathrm{~S}-4 \mathrm{P}_{1 / 2}$ transition. . 26

2.7. QI residuals for a cascade of subsequent decays . . . . . . . . . . 29

2.8. Cancellation of QI line shifts by weighted sum. . . . . . . . . . . . . 31

2.9. QI residuals for remaining initial state population . . . . . . . . . . . 33

3.1. Schematic overview of the hydrogen 2S-4P experiment . . . . . . . 36

3.2. Cryogenic hydrogen beam apparatus and XUV fluorescence detectors 37

3.3. Time-of-flight-resolved XUV detection . . . . . . . . . . . . . 38

3.4. Laser spectroscopy setup . . . . . . . . . . . . . . . . . . . . . . 39

3.5. Cancellation of the first order Doppler effect utilizing counter-propagating laser beams . . . . . . . . . . . . . . . . . . 41

3.6. Schematic view of the active fiber-based retroreflector (AFR) . . . . . 44

3.7. Estimation of AFR alignment sensitivity . . . . . . . . . . . . . 45

4.1. Observed transition frequency in the lab frame with and without Doppler suppression . . . . . . . . . . . . . . . . . . 48

4.2. Characterization of residual uncompensated first order Doppler shift . 49

5.1. Simulation of transverse velocity distributions . . . . . . . . . . 52

6.1. QI line shifts: experimental data (individual delays) . . . . . . . . 56

6.2. Measurement of apparent line shifts due to quantum interference . . . 57

6.3. Comparison of calculated QI line shifts for $2 \mathrm{~S}^{-} 4 \mathrm{P}_{1 / 2} \ldots \ldots \ldots$. . . . . 59

6.4. Power dependence of QI residuals . . . . . . . . . . . . . . . . 61

7.1. Experimental line centers for absolute frequency determination . . . . 64

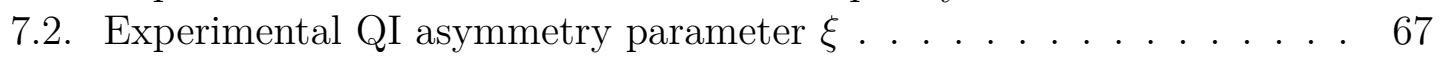

7.3. Test of expanded line shape model function using pseudo data . . . . 69 
7.4. Voigt vs. expanded line shape model: apparent QI line shifts . . . . . 70

7.5. Power dependence of line centers extracted from simulated line shapes 71

7.6. Relevant fine and hyperfine components for 2S-4P spectroscopy . . . 73

8.1. Determinations of $R_{\infty}$ and $r_{\mathrm{p}}$ (including tests of internal consistency) 77

8.2. Line scan sample data for the $2 \mathrm{~S}-6 \mathrm{P}$ transition . . . . . . . . . . 79

A.1. Re-evaluation of 2013 data $\left(2 \mathrm{~S}_{1 / 2}^{F=0}-4 \mathrm{P}_{1 / 2}^{F=1}\right.$ transition $) \ldots . . . . .84$

B.1. Voigt fits of the individual detectors' data . . . . . . . . . . 88

B.2. Observed line strengths of the $2 \mathrm{~S}-4 \mathrm{P}$ transitions . . . . . . . . . . . 89

B.3. Map of local detection efficiency (XUV detectors) . . . . . . . . . . . 91

C.1. Beam profiles: achromatic collimator vs. aspheric lens . . . . . . . . 96

C.2. Re-coupling loss AFR and residual Doppler shift . . . . . . . . . . . 97

C.3. AFR beam profiles: spheric aberration . . . . . . . . . . . . . 99

C.4. Doppler shift due to different AFR imperfections . . . . . . . . . 100

C.5. Characterization of AFR feedback . . . . . . . . . . . . . . . 102

E.1. 3D view of $2 \mathrm{~S}$ beam apparatus and detector configuration (2014) . . . 113

E.2. Technical drawing of 2014 beam apparatus (cut in x-y-plane) . . . . . 114

E.3. Technical drawing of 2014 beam apparatus (cut in y-z-plane) . . . . . 115 


\section{Introduction}

\subsection{The dawn of modern physics}

${ }^{1}$ Precision spectroscopy of atomic hydrogen, the simplest atomic system, has been one of the key tools for testing fundamental theories of light, matter and their interaction ever since the dawn of modern physics. Starting from the simple empirical formula by J.J. Balmer in 1885, that described the numerous distinct absorption and emission lines in the visible spectrum of atomic hydrogen, the theoretical description of the energy levels has been evolving over the last century, revolutionizing our basic understanding of nature not only once. In 1913, N. Bohr was the first to use the idea of matter waves to explain the discrete spectrum of atomic hydrogen [10]. In his well known planetary model of the atom, he proposed stationary states to be the ones where the orbit circumference matches an integer multiple of the electron de Broglie wave length.

Shortly thereafter, the emerging field of quantum mechanics was put on solid grounds by the first wave equation for matter waves formulated by E. Schrödinger. Solving the stationary Schrödinger equation of an electron bound to an infinitely heavy, point-like proton gives the same simple dependence of the energy levels $E_{n}$ on the principal quantum number $n$ as predicted by the Bohr model:

$$
E_{n}=-\frac{R_{\infty}}{n^{2}}
$$

where $R_{\infty}$ denotes the Rydberg constant. Increasing experimental accuracy and the observation of the fine structure in the hydrogen spectrum [11], i.e. the fact that the spectral lines in the Balmer series for example consist of doublets rather than single lines (see Fig. 1.1), which had not been predicted by the Bohr and Schrödinger theory, triggered the next major step in theory: P.A.M. Dirac reformulated the Schrödinger equation in 1928, including relativistic effects and introducing the electron spin $[12,13]$.

The Dirac theory withstood two decades of experimental tests, before W. E. Lamb and R. C. Retherford discovered a discrepancy with the theory in 1947 [14]: "The results indicate clearly that, contrary to theory [...], the $2^{2} \mathrm{~S}_{1 / 2}$ state is higher than the $2^{2} \mathrm{P}_{1 / 2}$ by about $1000 \mathrm{Mc} / \mathrm{sec}$ [1GHz]." According to the Dirac theory, these two states with equal principal quantum number $n=2$ and total angular momentum quantum number $j=1 / 2$ had been expected to be degenerate in energy although their orbital angular momentum quantum numbers are different ( $\mathrm{S}$ and $\mathrm{P}$ ). However,

${ }^{1} \mathrm{~A}$ major part of the content of this chapter has been published in [52] and was only slightly modified to fit the general structure of this thesis. 

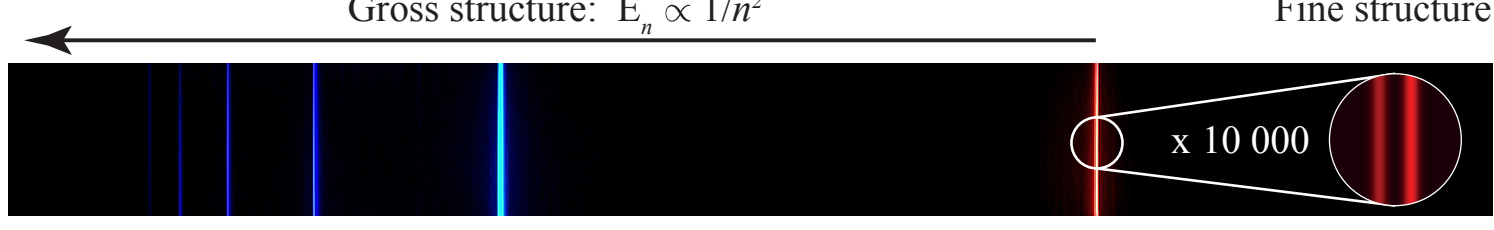

Figure 1.1.: Visible spectrum of atomic hydrogen [15]: The Balmer series, named after J. Balmer, who found an empirical formula to describe the spacing of the observed lines [16]. Later, the formula was generalized by J. Rydberg. Zooming in by a factor of about 10000 , the fine structure of the hydrogen spectrum becomes visible and was first explained by P. M. A. Dirac.

this discrepancy had not been caused by shortcomings of quantum theory itself, but rather by the fact that quantum mechanics had not been applied in its most rigorous way, as has been shown later by the pioneers of Quantum Electrodynamics (QED), E.A. Ühling, H.A. Bethe, R.P. Feynman and others. In particular, the way the quantum vacuum - both electronic and photonic contributions - affects the energy levels in atomic hydrogen had not yet been accounted for. Since then, QED passed every experimental test undertaken in the last 65 years and survived six orders of magnitude in improvement of experimental accuracy. Of course, several discrepancies between QED and experiments have shown up during this time, but all of them could be traced back to either neglected higher order terms in the calculations or underestimated experimental error bars.

\subsection{QED description of the hydrogen atom}

The theoretical description in the framework of QED expresses the energy levels in atomic hydrogen as a product of the Rydberg constant $R_{\infty}$ and a dimensionless function that represents the energies in Rydberg units. The latter is a function of the fine structure constant $\alpha$, the electron-to-proton mass ratio $m_{\mathrm{e}} / m_{\mathrm{p}}$ and the proton r.m.s. charge radius $r_{\mathrm{p}}$, while the Rydberg constant $\left(R_{\infty}=10973731.568539(55) \mathrm{m}^{-1}[4]\right)$ serves as a unit converter from Rydberg units to S.I. units. To be precise, even more parameters enter, e.g. the ratio of the electron mass to Planck's constant $m_{e} / h$. But at the current level of accuracy, those parameters play a minor role as will be discussed in the next paragraph. The energy levels can be written as:

$$
E_{n}=R_{\infty}\left(-\frac{1}{n^{2}}+f\left(\alpha, \frac{m_{e}}{m_{p}}, \ldots\right)+\frac{16 \pi^{2} m_{e}^{2} c^{2} \alpha^{2}}{3 h^{2} n^{3}} \delta_{\ell 0}\left\langle r_{\mathrm{p}}^{2}\right\rangle\right)
$$

Comparing Eqs. (1.1) and (1.2) illustrates a conceptual drawback of the developments sketched in the previous section: Not only became our description of nature more complicated over the decades, it also requires an entire set of parameters which can not be calculated from first principles. While for the non-relativistic Bohr and 


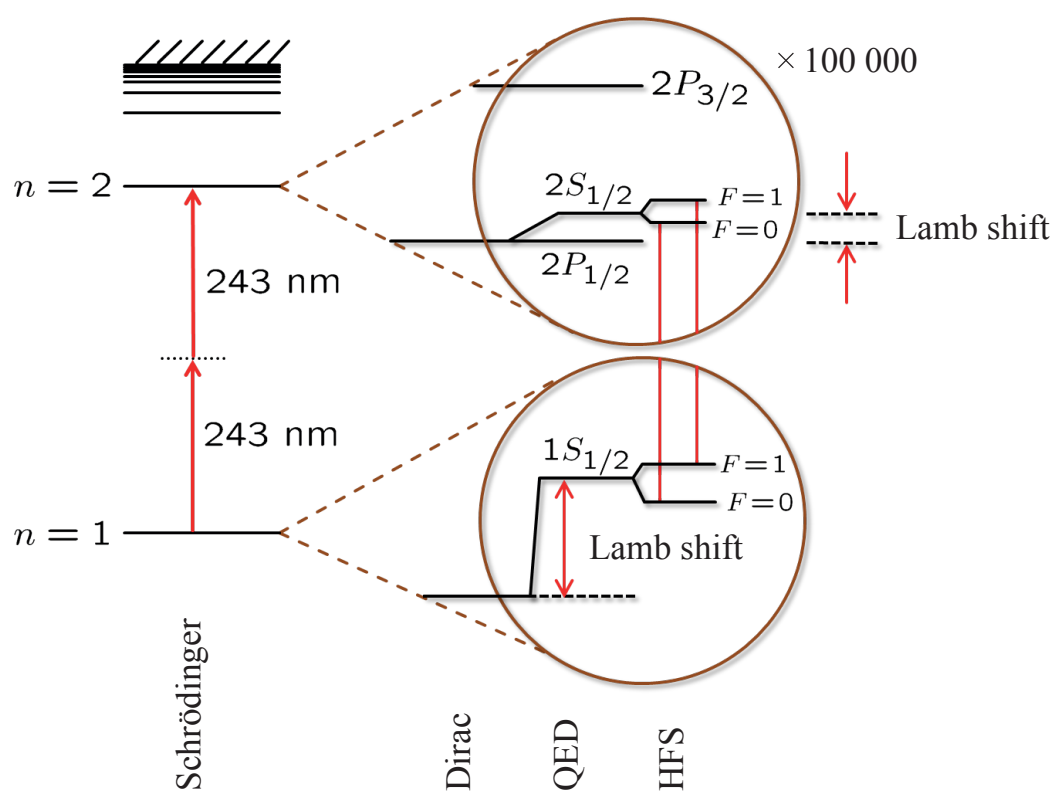

Figure 1.2.: Energy levels in atomic hydrogen. Going from left to right, the reader bridges 100 years of history in the theoretical description of the hydrogen atom. At the same time, the experimental accuracy increases by 10 orders of magnitude, comparing the observation of the fine structure in the Balmer series with state-of-the-art laser spectroscopy experiments, such as precision laser spectroscopy of the two photon $1 \mathrm{~S}-2 \mathrm{~S}$ transition $[6,7]$.

Schrödinger theory - reproduced by the leading order term in Eq.(1.2) - only the Rydberg constant was needed as an external input parameter, the full QED description needs additional parameters: Higher order terms add corrections to the simple nonrelativistic result, such as terms for the finite mass of the proton (relativistic recoil), the electron self energy and vacuum polarization summarized in $f\left(\alpha, m_{e} / m_{p}, \ldots\right)$ in Eq. (1.2). A compilation of all known contributions can be found in [4].

The last term in Eq.(1.2) is the leading order finite size correction. This correction arises due to the fact that the proton is not a point-like particle and accounts for the corresponding modifications of the proton's Coulomb potential seen by the electron depending on its particular orbit. To leading order, these corrections only affect states with a large overlap between electron wave function and the nucleus, i.e. for S-states where the electron is more likely to be found inside the proton. Since the finite size correction only adds a small contribution to the total energy and is entirely limited by the uncertainty of $r_{\mathrm{p}}$ (see e.g. Ref.[17]), the other parameters involved in this term can be taken from other experiments with sufficient accuracy.

\subsection{Determination of external input parameters}

In order to determine the remaining four parameters $\left[R_{\infty}, \alpha, m_{e} / m_{p}, r_{\mathrm{p}}\right]$, a combination of four measured transition frequencies in atomic hydrogen could in principle 
be used. Due to their higher sensitivity to individual parameters, however, precision measurements in other branches of physics offer more precise values for some of them: The fine structure constant can be extracted with an uncertainty of 3.7 parts in $10^{10}$ from the measurement of the free electron $g$-factor [18] and the corresponding QED expression [19]. This value is in agreement and three orders of magnitude more accurate than the value from hydrogen spectroscopy (see Ref.[4], Eq.(88)). Furthermore, mass ratios for particles which can be held in a Penning trap can be determined with relative uncertainties on the order of $10^{-12}$ by measuring their cyclotron frequencies [20]. This way, the electron-to-proton mass ratio has been determined with an uncertainty lower than from Eq.(1.2) given the accuracy of the data currently available from hydrogen spectroscopy [21].

\subsection{The proton size puzzle}

Following the arguments of the previous section, there are two parameters left to be determined to enable QED calculations competitive with current experimental accuracy: the Rydberg constant and the proton r.m.s. charge radius. These parameters can be extracted from a combination of two measured transition frequencies in hydrogen, while the comparison of results obtained from different pairs probes the internal consistency of the corresponding QED calculations.

The 1S-2S transition frequency with its relative frequency uncertainty of only 4.2 parts in $10^{15}$ is the most accurately measured transition frequency in atomic hydrogen $[6,7]$. Combining this result with each of the other measured transition frequencies listed in Table XI of the CODATA 2010 report [4], one obtains 15 pairs of values for $R_{\infty}$ and $r_{\mathrm{p}}$. Depicted in Fig.1.3 (a) and (b) are only the values for $r_{\mathrm{p}}$, since the corresponding plot for $R_{\infty}$ does not add much information due to the $96 \%$ correlation of the two parameters. A measure for the internal consistency of the values extracted is the Birge ratio $R_{B}$, i.e. the square root of the weighted mean quadratic deviation from the data set's weighted mean. For Gaussian distributed data, $R_{B}=1$ with a variance equal to $2 /(N-1)$ where $N$ is the number of data points. For the proton charge radii shown in Fig.1.3a), $R_{\mathrm{B}}=0.70(38)$ is compatible with Gaussian scatter of the results. Although this simple approach uses the 1S-2S transition frequency multiple times in the extraction process, its influence on the overall uncertainty of the results is negligible because its relative uncertainty is three orders of magnitude smaller than any of the other transition frequencies involved. The proper way to extract best estimates for the value pair $\left[R_{\infty}, r_{\mathrm{p}}\right]$ is to perform a least squares adjustment for the entire data set at once. The corresponding result of $r_{\mathrm{p}}=0.8764(89) \mathrm{fm}$ can be found in "adjustment \#8" in Table XXXVIII in the CODATA report [4].

Historically, $r_{\mathrm{p}}$ has been determined by elastic electron-proton scattering experiments. In the mid 1990s, though, hydrogen spectroscopy experiments and the corresponding theory became accurate enough to extract $r_{\mathrm{p}}$ with an uncertainty of about 


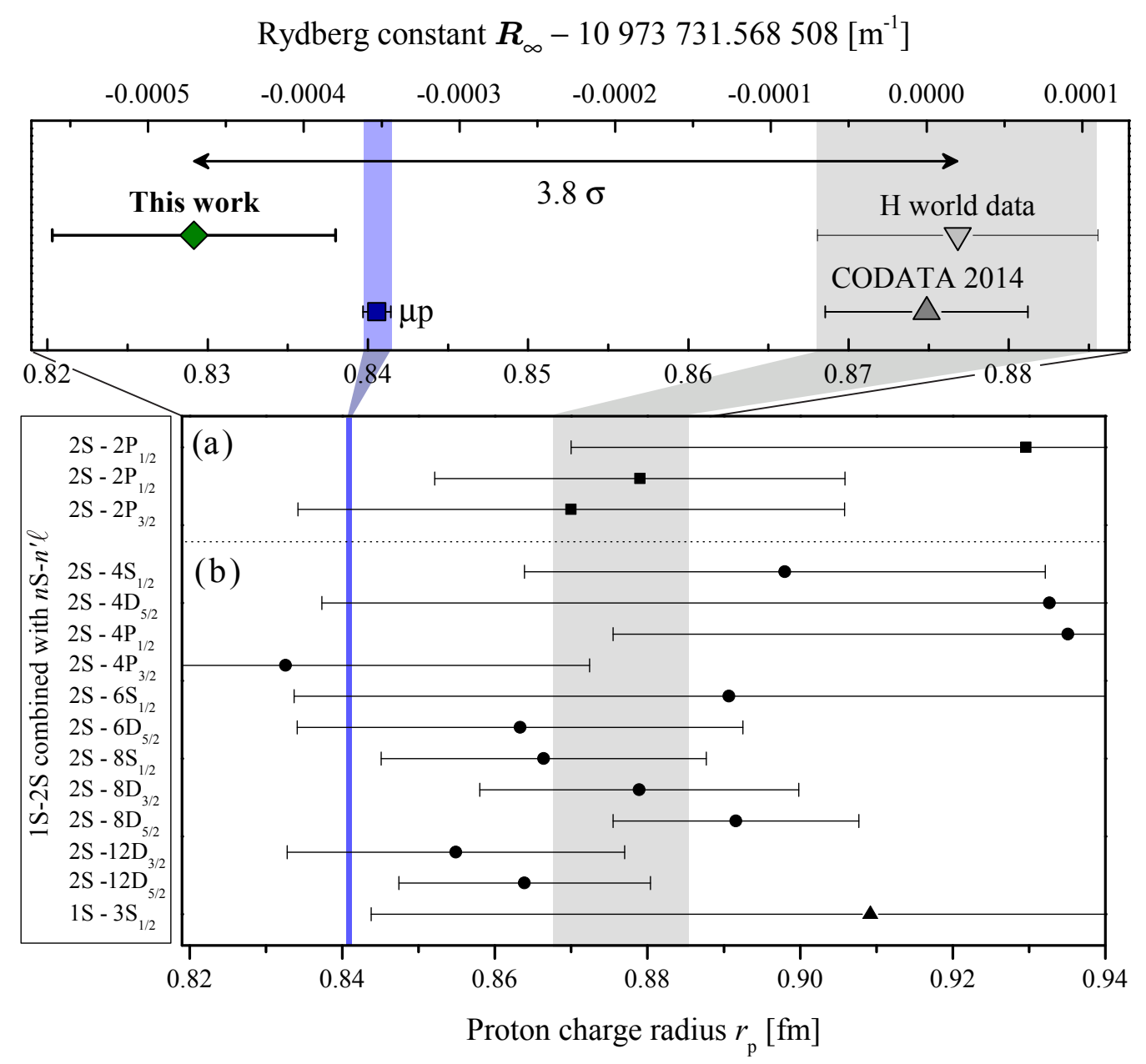

Figure 1.3.: Determinations of the Rydberg constant $R_{\infty}$ and proton charge radius $r_{p}$. Values $R_{\infty}=10973731.568037(91) \mathrm{m}^{-1}$ and $r_{p}=0.8293(91) \mathrm{fm}$ derived from this work (green diamond) and spectroscopy of muonic hydrogen (blue square, blue line in bottom plot) agree. A discrepancy of 3.8 combined standard deviations is found between this work and the world data of hydrogen $(\mathrm{H})$ spectroscopy (light gray triangle, gray shaded area in bottom plot) as well as the global adjustment of fundamental constants (gray triangle, CODATA2014). The $\mathrm{H}$ world data consists of 15 the individual measurements depicted in the bottom part. Experiments either probe transitions in the radio frequency (black squares in (a), historically called Lamb shift measurements) or optical domain (black points from the $2 \mathrm{~S}$ state (b), black triangle from the $1 \mathrm{~S}$ sate). In order to obtain a value pair $\left[R_{\infty}, r_{\mathrm{p}}\right]$, each transition frequency needs to be combined with another independent measurement, in the case shown, this is the $1 \mathrm{~S}-2 \mathrm{~S}$ transition frequency. In addition to $\mathrm{H}$ data, deuterium data (9 measurements) and a selection of electron-proton scattering data is included in the CODATA adjustment of fundamental constants [4]. Due to the strong correlation of $R_{\infty}$ and $r_{p}(96 \%)$, plots for the two parameters look essentially the same as indicated by the two different axes of abscissae on the top and bottom of the graph. 
1\%. The value extracted from hydrogen spectroscopy had been the most precise until in 2010, when laser spectroscopy of muonic hydrogen provided a new value ten times more accurate than any previous determination [1] (Fig. 1.3 top, blue square, $\mu-p)$. Muonic and electronic hydrogen are similar systems regarding their treatment in the framework of QED, but the muon is about 200 times heavier than the electron. Accordingly, the weight of the finite size correction is scaled up by $200^{2}$ relative to the other contributions in Eq.(1.2) when replacing $m_{e}$ by $m_{\mu}$ and by $200^{3}$, taking into account that another factor of $m_{e}$ is hidden within the Rydberg constant $R_{\infty}=\left(m_{e} \alpha^{2} c\right) / 2 h$. With this enhanced sensitivity, moderate accuracy in the determination of the $2 \mathrm{~S}_{1 / 2}-2 \mathrm{P}_{3 / 2}$ transition frequency and 5 out of the 12 known digits of the Rydberg constant are sufficient to obtain this impressive accuracy in $r_{\mathrm{p}}$. Meanwhile, the muonic hydrogen value has been confirmed and improved [2], yielding $r_{\mathrm{p}}=0.84087(39) \mathrm{fm}$, a value about $4 \%$ smaller than the values extracted from electronic hydrogen and elastic electron-proton scattering.

A $4 \%$ difference may seem small at first glance, but since electronic and muonic hydrogen are so similar in their theoretical description, Fig.1.3 reveals a highly unsatisfying situation: the proton charge radius (and the Rydberg constant) extracted from these two systems ( $\mathrm{H}$ world data and $\mu-\mathrm{p}$ ) are discrepant by four combined standard deviations. An even larger discrepancy of $7 \sigma$ is found when including deuterium spectroscopy data and a selection of electron-proton scattering data into the analysis as has been done in the "adjustment \#3" in the CODATA2010 report. This discrepancy is referred to as "the proton size puzzle". For a detailed discussion of possible ways to resolve the puzzle, we refer to the review [3], while the rest of this text will focus on the electronic hydrogen part.

\subsection{Improving the hydrogen input data}

Due to the tight link of the Rydberg constant and the proton charge radius, the proton size puzzle can be investigated by a new determination of the Rydberg constant with significantly increased accuracy. For the existing hydrogen data set, the longest lever arm on the puzzle can be found in improving measurements of transition frequencies starting from the metastable $2 \mathrm{~S}$ state. Squares in Fig. 1.3a) represent radio frequency measurements of the $2 \mathrm{~S}$ Lamb shift, i.e. the energy splitting between $2 \mathrm{~S}$ and $2 \mathrm{P}$ states, whereas points represent optical measurements in the visible domain. Each one of these individual measurements is compatible with the muonic hydrogen value within a few standard deviations, while the discrepancy of $4 \sigma$ only arises when the data is averaged.

On the other hand, to account for the discrepancy, the experimental value for the $1 \mathrm{~S}-2 \mathrm{~S}$ transition frequency which is used as a corner stone in the extraction would need to be shifted by 4000 experimental error bars and the value extracted from muonic hydrogen by $100 \sigma$. Expressed in line widths of the measured transitions, this corresponds to shifts of 40 and 4, respectively. Both experiments have been 
confirmed and improved recently, making this seem rather improbable. For the measured $2 \mathrm{~S}-n L$ transitions, the discrepancy corresponds to only small fractions of the respective line widths of about 1 part in $10^{3}$ or less.

Additional data input for these transitions is highly desirable to gain deeper insight into the discrepancy and is provided by this work. In addition, other hydrogen experiments are underway that use complementary techniques and transitions to contribute to the solution of the puzzle: e.g. an improved version of the classical Lamb shift experiment (2S-2P transition) is ongoing in Toronto [22], while experiments in Paris and Garching aim for an improved determination of the 1S-3S transition frequency $[23,24]$. The former can provide a value of $r_{\mathrm{p}}$ that does not rely on the 12 digits of accuracy of $R_{\infty}$ and the latter redundant data input complementary to the $1 \mathrm{~S}-2 \mathrm{~S}$ frequency.

There is no easy-to-follow recipe on how to choose a candidate transition to obtain additional input for the electronic hydrogen part of the proton radius puzzle. Each choice has its justification and goes along with a set of compromises a particular experiment has to deal with: Starting spectroscopy experiments from the $1 \mathrm{~S}$ ground state offers the advantage of having a large atomic population to start with. The drawback is that short wavelengths are required to drive these transitions. In practice, only two photon transitions with $n=2,3,4$ are addressable with simple means of frequency doubling or sum frequency conversion in commercially available non-linear optical materials $[23,24]$. Probing two photon transitions in general also provides the benefit of small natural line widths, while this advantage comes at the price of high excitation light powers being required to obtain sufficient event rates. Not only is this a technical challenge for transitions with a large energy gap to bridge, it also requires a detailed study of excitation light intensity dependent effects. Especially the ac Stark effect is a leading order systematic effect in past $2 \mathrm{~S}-n S / D$ experiments.

In contrast to that, the large dipole matrix elements of one photon transitions have to be traded in for the sensitivity to the first order Doppler effect which can be efficiently suppressed by two photon absorption. In both cases, a larger principal quantum number $n$ of the excited state leads to narrower transitions. But the sensitivity to external fields increases and the dc Stark effect is of particular concern, because it is proportional to $n^{7}$ in first approximation. In the end, only the accuracy and reliability of the various systematic studies are the most critical ingredient for any choice. The the apparatus developed in the course of this thesis for the $2 \mathrm{~S}-4 \mathrm{P}$ transition is well adapted for this task. Its main advantages are summarized in the following section.

\subsection{Advantages of the Garching $2 \mathrm{~S}-4 \mathrm{P}$ apparatus}

The difference in the corresponding parameter pairs $\left[R_{\infty}, r_{\mathrm{p}}\right]$ determined from electronic and muonic hydrogen leads to a difference in the predicted $2 \mathrm{~S}-4 \mathrm{P}$ transition 
frequency of only about $8.9 \mathrm{kHz}$. The uncertainties of the most precise experimental determination though amount to $15 \mathrm{kHz}$ for the $2 \mathrm{~S}-4 \mathrm{P}_{1 / 2}$ transition and $10 \mathrm{kHz}$ for the $2 \mathrm{~S}-4 \mathrm{P}_{3 / 2}$ fine structure component [5]. Hence a reduction of the experimental uncertainties by a factor of five is required to reach the desired level of accuracy. Due to the comparably large natural line width of $12.9 \mathrm{MHz}$ of the $2 \mathrm{~S}-4 \mathrm{P}$ lines, this corresponds to determining the line center of the transition to much better than 1 part in $10^{3}$ of the line width.

During this thesis, the Garching 1S-2S experiment was extended to accommodate a high resolution spectroscopy setup for the $2 \mathrm{~S}-4 \mathrm{P}$ one photon transition (Sec. 3). The proven 1S-2S apparatus is now used as a well-controlled source of $5.8 \mathrm{~K}$ cold atoms in the metastable $2 \mathrm{~S}_{1 / 2}^{F=0}$ state. This setup has several advantages and very different systematic limitations, compared to the previous most precise hydrogen and deuterium measurements.

The state-selective optical excitation of the $2 \mathrm{~S}_{1 / 2}^{F=0}$ state preserves the low thermal velocity of the sample atoms originating from a cryogenic source. At the same time it drastically simplifies the subsequent dynamics of the $2 \mathrm{~S}-4 \mathrm{P}$ excitation compared to electron-impact-excitation, which populates all 2S sublevels and has been used in past experiments. A beam of meta-stable atoms is obtained with a mean thermal velocity around $\langle v\rangle=300 \mathrm{~m} / \mathrm{s}$, ten times smaller than using electron-impact-excitation.

In addition, an active fiber-based retroreflector has been developed within this thesis [25] and provides a high level of compensation of the first order Doppler effect (AFR, Sec. 3.5). The suppression is verified by probing subsamples of atoms with different mean velocity provided by a time-of-flight resolved detection scheme. The transition is driven by two phase-retracing anti-parallel laser beams, leading to Doppler shifts of opposite sign and equal amplitude for atoms being excited by either of the beams. The residual uncertainty due to the first order Doppler effect has been evaluated to be $1.6 \mathrm{kHz}$ in a separate study (see Sec. 4).

Avoiding electron-impact excitation and high excitation light powers, our measurement is essentially unaffected by the systematic effects dominating in the previous two photon experiments. In particular, these are the AC Stark shift $(<0.1 \mathrm{kHz})$, the second order Doppler shift $(<0.2 \mathrm{kHz})$ and line shifts due to optical pumping and unresolved hyperfine components $(<0.1 \mathrm{kHz})$ which typically amount to tens of kilohertz in these measurements (see for instance [26] and references therein).

With the clean 2S-4P excitation dynamics provided by the well-controlled optical $2 \mathrm{~S}$ excitation (see Fig. 2.4), we are also able to study apparent line shifts due to quantum interference of neighboring atomic transitions detailed in Sec. 6. This systematic effect has been brought to the attention of the atomic spectroscopy community only recently [8] and was hence ignored in all previous hydrogen and deuterium measurements used in the CODATA adjustment [4].

The absolute transition frequency $\nu_{2 S-4 P}$ determined in this work is used in combination with measurement of the 1S-2S transition frequency to extract the Rydberg 
constant and the proton charge radius via Eq. (1.2):

$$
\begin{aligned}
R_{\infty} & =10973731.568037(91) \mathrm{m}^{-1} \\
r_{p} & =0.8293(91) \mathrm{fm} .
\end{aligned}
$$

These values are as accurate as the ones that can be extracted from the aggregate world data of all precision electronic hydrogen data shown in Fig. 1.3 [4]. While there is a discrepancy of $3.8 \sigma$ to the latter, our results are in agreement with the ones derived from laser spectroscopy of muonic hydrogen $[1,2]$. Implications of these findings for the proton size puzzle [3] are discussed in Sec. 8. 



\section{Theory of quantum interference from neighboring atomic resonances}

Line shape distortions due to quantum interference of neighboring atomic resonances lead to a break-down of the simple approximation of natural atomic line shapes by Lorentz functions. They result in apparent geometry-dependent shifts of the observed line centers if not properly taken into account when fitting the experimental data. These distortions have been calculated to cause significant shifts in precision spectroscopy experiments in helium even at a large separation of two neighboring resonances up to 10,000 natural line widths [8, 9, 27-29].

Experimental evidence for the accompanying shifts in precision spectroscopy, however, only existed for the partially unresolved hyperfine components in $\mathrm{Li}^{6,7}$ up to now [30, 31]. For the first time, the crucial importance of quantum interference effects is demonstrated in this thesis (Sec.6) for resonances separated by as much as 100 natural line widths. We anticipate here that the maximally observed differences in the line centers determined by usual Voigt fits of our $2 \mathrm{~S}-4 \mathrm{P}$ data can be as large as $\pm 51.5 \mathrm{kHz}$. This is large compared to the proton size puzzle which corresponds to $8.9 \mathrm{kHz}$ for the $2 \mathrm{~S}-4 \mathrm{P}$ frequency. A detailed understanding of this systematic is therefore mandatory.

Up to now, the common procedure of dealing with these effects has been to calculate corrections to the experimental data using the numerical solution of the master-equation describing the atomic excitation dynamics (Sec. 2.2.3). This approach requires the use of powerful computation centers as well as detailed knowledge about the experimental geometry and detection efficiency. To guide the physical intuition which may partially be lost when dealing with the complex master-equation approach, we first introduce a simple example from classical electrodynamics. Subsequently, a simple line shape model is derived that accounts for the line distortions, extracts the unperturbed line center independent of the experimental geometry and renders the correction of experimental data by simulation results unnecessary (Sec. 2.2.1). It is used for the determination of the $2 \mathrm{~S}-4 \mathrm{P}$ absolute transition frequency in Sec. 7. The theoretical descriptions using a perturbative approach and the more powerful aforementioned master-equation approach are discussed in Sec 2.2.2 and Sec. 2.2.3, respectively. 


\subsection{Classical analog: Two driven antennas}

Consider two classical antennas with eigenfrequencies separated by $\Delta$ and identical damping constants $\Gamma$ which are driven by an electric field $\vec{E}_{i n}(t)=\vec{E}_{0} e^{i w t}$. The antennas may point in different directions denoted by the unit vectors $\hat{d}_{1}$ and $\hat{d}_{2}$. Using the usual susceptibilities the resulting dipole moment is given by:

$$
\vec{D}(\omega)=\frac{\left(\hat{d}_{1} \cdot \vec{E}_{0}\right) \hat{d}_{1}}{\omega+i \Gamma}+\frac{\left(\hat{d}_{2} \cdot \vec{E}_{0}\right) \hat{d}_{2}}{\omega-\Delta+i \Gamma}
$$

Placed at the origin, this dipole moment generates an electric field $\vec{E}_{\text {out }}$ at the location $\vec{r}$. The observed power spectrum $P(\omega, \vec{r})$ is proportional to its square modulus [32]:

$$
P(\omega, \vec{r}) \propto\left|E_{\text {out }}\right|^{2} \propto\left|\frac{\vec{r} \times \vec{D}(\omega) \times \vec{r}}{r^{3}}\right|^{2} \propto D^{2}(\omega) \frac{1-\cos \left(\theta_{d}\right)^{2}}{r^{2}},
$$

with $D^{2}(\omega)=|\vec{D}(\omega)|^{2}, r=|\vec{r}|$ and the detection angle $\theta_{d}$ defined in spherical coordinates (similar to Fig. 2.3). The observed spectrum consists of two real valued Lorentzians and a non-Lorentzian cross term:

$$
\begin{aligned}
D^{2}(\omega) & =\frac{D_{1}^{2}}{\omega^{2}+(\Gamma / 2)^{2}} \\
& +\frac{D_{2}^{2}}{(\omega-\Delta)^{2}+(\Gamma / 2)^{2}} \\
& +2 \vec{D}_{1} \cdot \vec{D}_{2} \frac{\omega(\omega-\Delta)-(\Gamma / 2)^{2}}{\left(\omega^{2}+(\Gamma / 2)^{2}\right)\left((\omega-\Delta)^{2}+(\Gamma / 2)^{2}\right)}
\end{aligned}
$$

where $\vec{D}_{j}=\left(\hat{d}_{j} \cdot \vec{E}_{0}\right) \hat{d}_{j}$ denote the respective dipole amplitudes. The latter depends on the relative orientation of the dipoles and vanishes for orthogonal alignment, i.e. for $\hat{d}_{1} \cdot \hat{d}_{2}=0$. Approximating a multiplet of neighboring atomic resonances by real-valued Lorentzian functions neglects this cross-term. The problem is illustrated in Fig. 2.1: If we ask the question to which extent the line center of the first resonance at $\omega_{1}$ extracted by a Lorentz fit is influenced by the presence of a second resonance at $\omega_{2}$, we get vastly different answers. We define the apparent shift due to the cross-term by the difference of the line center $\omega_{0}^{\mathrm{LOR}}$ extracted from a Lorentz fit and the position of the true line center $\omega_{0}$ :

$$
\Delta \omega_{\mathrm{CT}}=\omega_{0}^{\mathrm{LOR}}-\omega_{0} .
$$

The shifts introduced by the line shape modifications due to the cross-term (red line) are orders of magnitude larger than the ones expected from the simple Lorentz approximation (black dashed line) and of special importance at separations of the two resonances that are large compared to the corresponding line widths. 

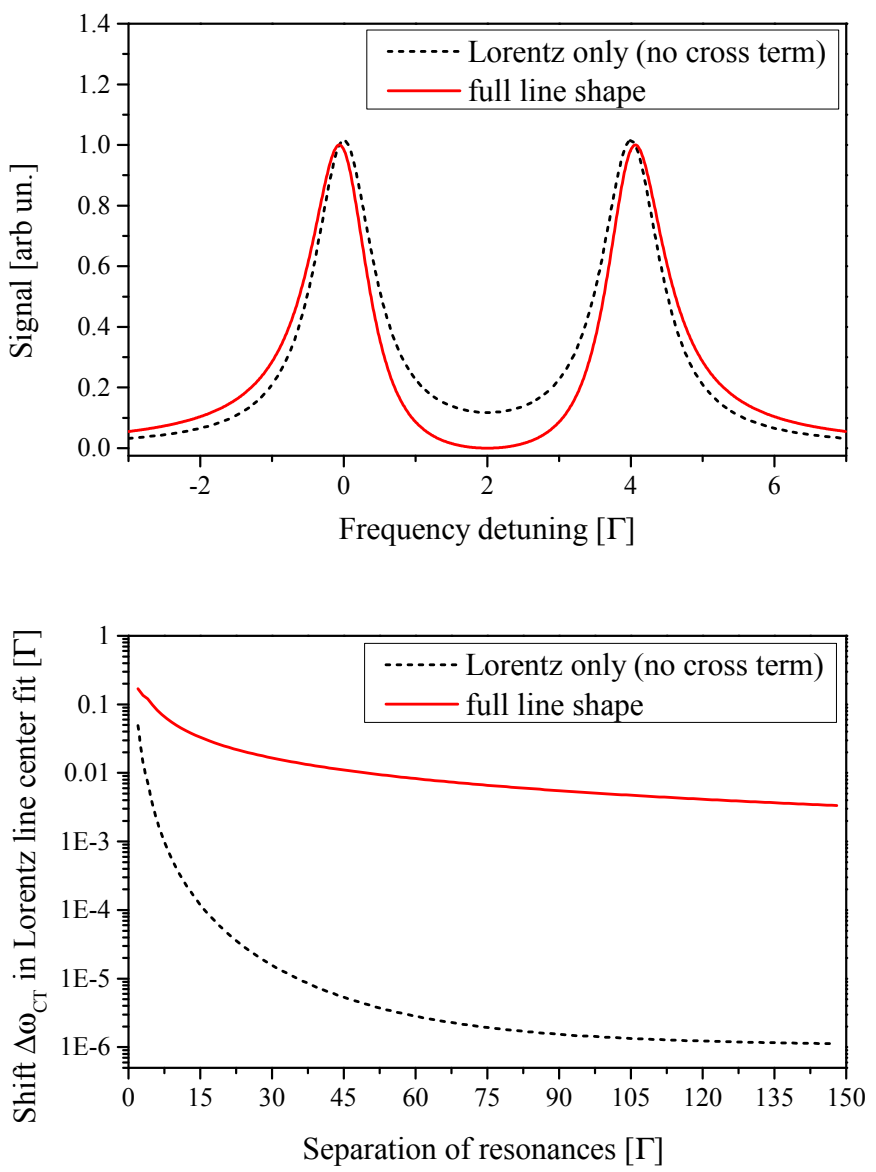

Figure 2.1.: Classical ana$\log$ to apparent line shifts due to quantum interference from neighboring resonances. Top: line shapes obtained form Eq. (2.2) with (red line) and without (black dashed line) the coherent cross term (third term in Eq. (2.3)). The frequency detuning is measured in units of the natural line width $\Gamma$ and in the case shown, the separation of the two resonances is $4 \Gamma$. Bottom: Shifts $\Delta \omega_{\mathrm{CT}}$ in the line centers obtained from a Lorentz fit to one of the spectral components in the power spectrum above. The influence of the second resonance on the extracted Lorentz fit center is orders of magnitude larger in case of the full model (red), especially at large separations of the two resonances.

Treating the second resonance at $\omega_{2}$ as a perturbation to the resonance at $\omega_{1}$, the full line shape in Eq. (2.2) can be approximated around the resonance position [33]:

$$
D^{2}(\omega) \approx \frac{a_{1}}{\omega^{2}+(\Gamma / 2)^{2}}+a_{2} \omega+\frac{a_{4} \omega}{\omega^{2}+(\Gamma / 2)^{2}} .
$$

The constant offset $a_{3}$ in [33] has been dropped since it does not influence the position of the observed line center and is usually absorbed in a fit of a flat background. The first term denotes the line shape LS1 of the isolated, unperturbed resonance at $\omega_{1}$, while the other two terms represent perturbations due to the second resonance (LS2). The second term accounts for LS1 sitting on the far-reaching wing of LS2 (incoherent line pulling). Compared to the third term that accounts for interference between LS1 and LS2 and leads to dispersive shaped distortions of the line (coherent line pulling), the second term is small. The geometry dependence in Eq. (2.2) is absorbed in the parameters $a_{1}, a_{2}$ and $a_{4}$. Neglecting the third term, i.e. modeling the natural line shape as a sum of real valued Lorentzians, leads to geometry dependent shifts of the half width points of [33]:

$$
\Delta \omega=\frac{a_{2} \Gamma^{4}}{8 a_{1}}+\frac{a_{4} \Gamma^{2}}{4 a_{1}} \approx-\frac{\vec{D}_{1} \cdot \vec{D}_{2}}{2 D_{1}^{2}} \frac{\Gamma^{2}}{\Delta}+\mathcal{O}\left(\frac{\Gamma^{4}}{\Delta^{3}}\right)
$$




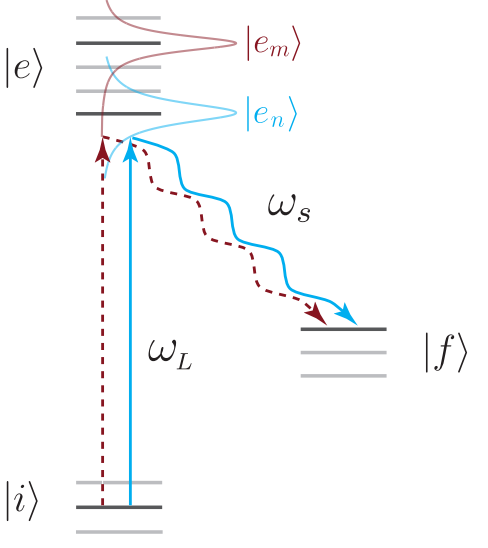

Figure 2.2.: Level scheme for quantum interference from distant neighboring resonances. Transitions from the initial states $|i\rangle$ to the final states $|f\rangle$ via excited states $|e\rangle$ are introduced by electromagnetic radiation at frequency $\omega_{L}$. Quantum mechanical interference takes place between coherently excited path ways leading from a common initial state to a common final state, as indicated by the blue solid (via near-resonant state $\left|e_{n}\right\rangle$ ) and red dashed line (via distant neighboring state $\left.\left|e_{m}\right\rangle\right)$. Natural line widths of the involved states are indicated by Lorentz functions.

We note that Eq. (2.6) changes sign when replacing $\vec{D}_{1}$ and $\vec{D}_{2}$ and $\Delta$ by $-\Delta$, i.e. the two resonances are shifted in opposite directions and the ratio of the shifts is given by the ration of their dipole amplitudes. Using this fact, it can be shown that by calculating the center of gravity of the two resonances, the residual shift can be reduced to:

$$
\Delta \omega_{Q I}^{(\mathrm{cg})}=0+\mathcal{O}\left(\frac{\Gamma^{4}}{\Delta^{3}}\right) .
$$

This important finding can also be utilized for two interfering atomic resonances and will be discussed as one possible way of canceling the apparent shifts in Sec. 2.4.3.

\subsection{Atomic line shape models}

For real atomic systems, proper modeling of the natural line shapes is more cumbersome than for the simple toy model in the previous section. The same arguments apply, however. We consider a multi level atom as shown in Fig. 2.2. Transitions from the initial states $|i\rangle$ to the final states $|f\rangle$ via excited states $|e\rangle$ are induced by electromagnetic radiation at frequency $\omega_{L}$. The frequency of the scattered light is $\omega_{s}$. Note that energy conservation has to be obeyed, i.e. the energy gain of the atom equals energy loss of the photon and vice versa: $\omega_{f i}=\omega_{s}-\omega_{L}$. Although the frequency $\omega_{L}$ is closely tuned to resonance with $\left|e_{n}\right\rangle$, the excitation amplitude to one or more off-resonant levels $\left|e_{m}\right\rangle$ is non-zero as indicated by Lorentz functions symbolizing the natural line shape of the isolated resonances. Quantum mechanical interference takes place between these different path ways leading from a common initial state to a common final state and is indicated by the blue solid and red dashed line. States $|i\rangle$ and $|f\rangle$ may be identical, which is important to consider if the excitation light power is high enough so that optical pumping and saturation effects become important (see Sec. 6.2).

An expanded line shape model analogous to the one in the previous section is introduced in Sec 2.2.1 and Sec.2.3.2. In addition, two approaches used to model the effects of QI line shifts are a perturbative approach similar to the description in 
[31] and the numeric integration of the optical Bloch equations (OBEs) describing the dynamics of our system following [34]. Both approaches follow a semiclassical ansatz, i.e. the applied electromagnetic field is treated classically. The perturbative approach is an analytic approximation of the involved dynamics. It is easy to implement and computationally inexpensive. However, this approach is incapable of describing multiple excitations of the same atom, such as optical pumping and saturation effects. The relative simplicity of the perturbative approach makes it generally less prone to algebraic and numeric errors, can help to guide the physical intuition and make it a valuable counterpart to the OBE description. The treatment of a real atomic system via OBEs is generally more powerful because optical pumping and saturation are included automatically and any additional external influence on the experimental line shape, e.g. Doppler broadening, can be included in the same framework. Yet the number of equations to solve is $N^{2}$, if $N$ is the number of levels involved. Computationally intensive calculations and a certain loss of intuition are the accompanying price. We will discuss the limits of the perturbative description of our experimental data in Sec. 6.2.

\subsubsection{Expanded line shape}

Analogous to the discussion in Sec.2.1, an efficient way of dealing with quantum interference line shifts in experimental data is the use of the expanded line shape in Eq. (2.5). We show in Sec. 2.3.2 that the corresponding shifts can be expressed in simple analytic form in the perturbative regime, i.e. when the effects of saturation and optical pumping may be neglected. The expanded line shape is used to study basic properties of the apparent quantum interference line shifts in Sec. 2.4 and has been validated by comparison to the line shape models discussed in the following sections.

To also take into account Gaussian broadening mechanisms, such as the atomic beam divergence in our experiment (Sec. 3), the expanded line shape (Eq. (2.5)) is convoluted with a Gaussian of width $\Gamma_{G}$ (1/e width). Omitting the small linear term proportional to $a_{2}(\Gamma / \Delta \ll 1)$ and similar to the description in [35], this yields:

$$
\mathcal{F}(\nu)=A\{\Re[w(z)]+\xi \Im[w(z)]\},
$$

where $w(z)$ denotes the complex error function of the argument $z=\left(\omega-\omega_{0}\right) / \Gamma_{G}+$ $i \Gamma / 2 \Gamma_{G}$. Analogous to Eq. (2.5), the line shape in Eq. (2.8) consists of a Voigt profile and a dispersive shaped perturbation. The parameter $\xi=a_{4} \Gamma / 2 a_{1}$ measures the amplitude of this perturbation relative to the observed line strength.

It will become clear in the following sections, that accurate simulations of the apparent line shifts due to quantum interference can be extremely challenging. This is mostly caused by the fact, that accurate simulations require a precise description of the excitation dynamics as well as experimental geometry factors which may both be difficult to quantify and validate. 
The line shape model presented in this section does not require this kind of external input. Instead, line shape distortions due to quantum interference (and other possible dispersive shaped distortions, see Sec. 7.4.2) are accounted for by the fit parameter $\xi$. This results in a robust extraction of the unperturbed line center as shown later in the text (Sec. 7.4.3), without the need of sophisticated (and potentially error-prone) numerical simulations.

The expanded line shape model in Eq. (2.8) is therefore one of the key components in the data analysis of the $2 \mathrm{~S}-4 \mathrm{P}$ absolute frequency measurement in Sec. 7. Simulation data, on the other hand, are used to gain intuition about the general properties of QI line shifts (Sec. 2.4) and the effects involved in the specific experimental implementation (Sec. 6).

\subsubsection{Perturbative approach}

The theoretical starting point for the perturbative approach is the Kramers-Heisenberg relation [36]. Similar to the power spectrum in our simplified example in Eq. (2.2), the scattering rate $R_{i \rightarrow f}^{\nu, \eta}$ is calculated by a coherent sum over all possible path ways leading from an initial state $|i\rangle$ to a final state $|f\rangle$ via all possible excited states $|e\rangle$. The scattering matrix elements $\mathcal{S}_{e, i \rightarrow f}^{\nu, \eta}$ can be written as:

$$
\mathcal{S}_{e, i \rightarrow f}^{\nu, \eta}=\frac{D_{e, f}^{\nu} D_{i, e}^{\eta}}{\omega_{e f}-\omega_{L}-i \Gamma_{e} / 2} .
$$

We chose a representation of the scattering rate $R$ in spherical components $(\eta, \nu)=$ $-1,0,+1$ which is favorable for the description of the Zeeman substructure of the atomic levels. $\Gamma_{e}$ denotes the inverse life time of states $|e\rangle$. The dipole moments are given by $D_{f e}^{\eta}=q\left\langle f\left|\vec{\epsilon}_{\eta} \cdot \vec{r}\right| e\right\rangle$ where $q$ is the elementary charge and $\vec{\epsilon}_{\eta}$ are the spherical unit vectors, expressed in Cartesian coordinates as $\vec{\epsilon}_{0}=(0,0,1)$ and $\vec{\epsilon}_{ \pm 1}=-( \pm 1, i, 0) / \sqrt{2}[37$, eq. (4.111)], and $\vec{r}$ the position operator. The physical meaning of $R_{i \rightarrow f}^{\nu, \eta}$ is the amount of radiation scattered by the atom from one spherical component $\eta$ of the exciting dipole operator $D_{i, e}^{\eta}$ into the spherical component $\nu$ of the emitting dipole operator $D_{i, e}^{\eta}$ via all excited states $|e\rangle . R_{i \rightarrow f}^{\nu, \eta}$ is calculated via:

$$
R_{i \rightarrow f}^{\nu, \eta}=\frac{\pi E_{L}^{2} \omega_{s}^{3}}{h^{3} c^{3} \epsilon_{0}}\left|\sum_{e} \mathcal{S}_{e, i \rightarrow f}^{\nu, \eta}\right|^{2} .
$$

$E_{L}$ denotes the electric field strength of the incident electromagnetic wave, $\omega_{s}$ the frequency of the scattered light, $h$ Planck's constant, $c$ the vacuum speed of light and $\epsilon_{0}$ the the permittivity of free space. Here, we use the convention that $\omega_{a b}=2 \pi\left(E_{a}-E_{b}\right) / h$ with $E_{a, b}$ being the energies of states $|a\rangle$ and $|b\rangle$ respectively.

The spherical components $\nu=-1,0,+1$ correspond respectively to $\sigma_{-}, \pi$ and $\sigma_{+}$dipole radiation modes. The far-field electric field $\vec{E}_{F F}$ in a certain direction of detection $\vec{r}_{d}$ can be expressed as:

$$
\vec{E}_{F F} \propto\left(\vec{r} \times \vec{\epsilon}_{\nu}\right) \times \vec{r}
$$




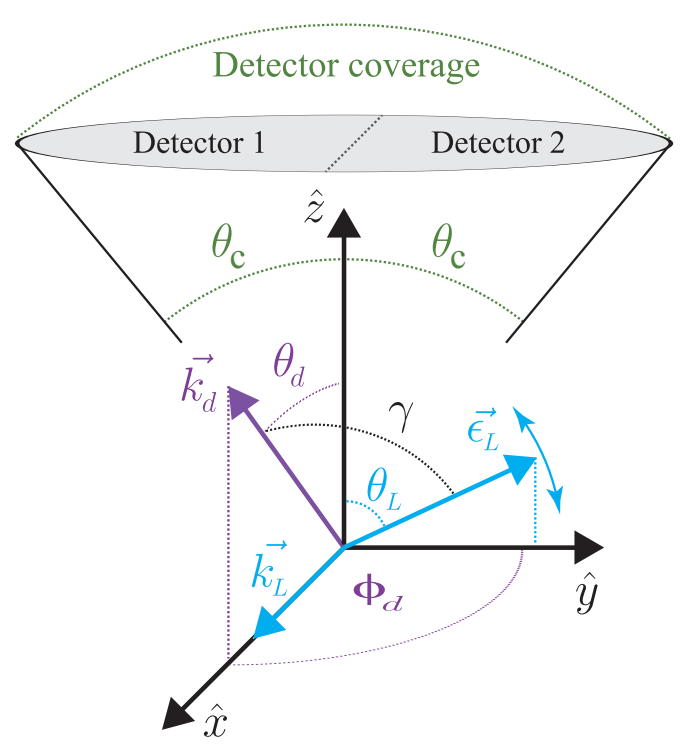

Figure 2.3.: Coordinate system used throughout this text. The excitation and emission point is located at the origin. Spherical coordinates $(\theta, \phi)$ are used to describe the detected signal in direction of $\overrightarrow{k_{d}}$. In the experiment (Sec. 3), laser light propagates along the x-axis with wave vector $\vec{k}_{L}$ and linear laser polarization $\vec{\epsilon}_{L}$. A rotationally symmetric detector along the z-axis and opening angle $\theta_{c}$ is split along the $\mathrm{x}$-z-plane to enhance sensitivity to quantum interference line shape modifications upon rotation of the linear laser polarization $\left(\theta_{L}\right)$ in the y-z-plane. The atomic beam axis coincides with the y-axis of the coordinate system.

We use this to calculate the intensities emitted into the three spherical components as a function of incident light polarization $\eta$ and emission direction in spherical coordinates $(\theta, \phi)$ :

$$
\begin{aligned}
I_{-1, \eta}(\theta) & =\frac{1}{2}\left(\cos ^{2}(\theta)+1\right) \sum_{f}\left|\sum_{e} \mathcal{S}_{e, i \rightarrow f}^{-1, \eta}\right|^{2}, \\
I_{0, \eta}(\theta) & =\sin ^{2}(\theta) \sum_{f}\left|\sum_{e} \mathcal{S}_{e, i \rightarrow f}^{0, \eta}\right|^{2}, \\
I_{+1, \eta}(\theta) & =\frac{1}{2}\left(\cos ^{2}(\theta)+1\right) \sum_{f}\left|\sum_{e} \mathcal{S}_{e, i \rightarrow f}^{+1, \eta}\right|^{2} .
\end{aligned}
$$

The coordinate system used throughout this text is shown in Fig. 2.3. In the chosen representation, all the radiation modes are rotationally symmetric about the z-axis and no $\phi$ - dependence shows up in the formulas above. We include the possibility of having multiple final states by summing over $f$. Note, that interference only occurs, between pathways that lead from a common initial state to a common final state via different excited states. This can be shown analytically by use of the dipole selection rules (see App. D.1). Accordingly, the contributions from decays to different final states are summed up incoherently in Eqs. (2.12) - (2.14). We will come to this point once again in the discussion of the damping terms for the master-equation approach. Furthermore, path ways do not interfere for $\nu \neq \nu^{\prime}$ and the corresponding intensities $I_{\nu, \eta}(\theta)$ are added up incoherently, too. For a formal proof, see App. D.3.1.

For linear laser polarization along the $z$-axis or circular polarization about the $z$-axis, the intensity distribution of the scattered light can be calculated with $\eta=0$ 
and $\eta= \pm 1$, respectively, using (2.12) or (2.13). Any other polarization vector $\overrightarrow{\epsilon_{L}}$ has to be expressed in terms of its spherical components and the dipole moment $D_{i, e}^{\eta}$ in (2.9) is replaced by the scalar product:

$$
\vec{D}_{i, e} \cdot \overrightarrow{\epsilon_{L}}=\sum_{\eta}\left[D_{i, e}^{\eta}\right]^{*} \epsilon_{\eta}
$$

\subsubsection{Master-equation approach}

The starting point for the numerical integration of the optical Bloch equations (OBEs) is the master-equation for the interaction of a multi-level atom with an electromagnetic field using the density matrix formalism [34, 38]. The time evolution of the density matrix $\rho$ is given by:

$$
\frac{\partial \rho}{\partial t}=\frac{1}{i \hbar}[H, \rho]-\mathcal{L} \rho
$$

Applying the rotating wave approximation, the Hamilton operator $H=H_{0}+H^{\prime}$ can be written as a sum of the atomic Hamilton operator

$$
H_{0}=\sum_{k=1}^{N_{1}} \hbar \omega_{k}|k\rangle\langle k|
$$

and the Hamiltonian for the interaction with the electromagnetic wave:

$$
H^{\prime}=\frac{\hbar}{2} \sum_{l=1}^{N_{2}}\left[\Omega_{l} S_{l}^{+} e^{-i \omega_{L} t}+\Omega_{l}^{*} S_{l}^{-} e^{i \omega_{L} t}\right]
$$

$N_{1}$ is the number of levels $|k\rangle$ involved in the dynamics and $\hbar \omega_{k}$ their respective energies. The index $l$ corresponds to a unique enumeration of all dipole allowed transitions with a total number of $N_{2}$. Following the notation in [34], we introduced the operator $S_{l}^{+}=|i\rangle\langle e|$, where states $|i\rangle$ and $|e\rangle$ correspond to transitions labeled by $l$ with Rabi frequencies $\Omega_{l}^{\prime}=E_{L} D_{i, e} / \hbar$. Likewise, $S_{l}^{-}=|e\rangle\langle i|=\left[S_{l}^{+}\right]^{*}$. Spatially varying phase terms $e^{ \pm i \Phi}$ in the electric field, e.g. due to wave front curvature of the excitation light, have been absorbed in the complex Rabi frequencies $\Omega_{l}=\Omega_{l}^{\prime} e^{ \pm i \Phi}$.

We have grouped the decay terms in Eq.(2.16) into the Lindblad operator $\mathcal{L}$, given by [34, 38]:

$$
\mathcal{L} \rho=\frac{1}{2} \sum_{m, n=1}^{N_{3}} \Gamma_{m n}\left\{S_{m}^{+} S_{n}^{-} \rho-2 S_{n}^{-} \rho S_{m}^{+}+\rho S_{m}^{+} S_{n}^{-}\right\} .
$$

The operators $S_{p}^{ \pm}$used in Eq. (2.19) are defined analogous to the ones above, but for the allowed decays from the excited states $|e\rangle$ to the final states $|f\rangle$ labeled by another unique enumeration $p=1,2, \ldots N_{3}$. The coefficients $\Gamma_{i j}$ are given by $\Gamma_{i j}=\sqrt{\Gamma_{i} \Gamma_{j}} \overrightarrow{\epsilon_{i}} \cdot \overrightarrow{\epsilon_{j}}$ where $\Gamma_{i}$ are the decay constants for transition $i$ and $\overrightarrow{\epsilon_{i}}$ are 
the spherical unit vectors along the corresponding dipole moments. Note, that due to the summation over two running indices $m$ and $n$ in Eq. (2.19), the decay of a certain excited state $\left|e^{\prime}\right\rangle$ does not only depend on the population in $\left|e^{\prime}\right\rangle$ itself (normal damping), but also on the coherence relative to other excited states $|e\rangle$. The latter is referred to as both, quantum interference and cross-damping. We re-write Eq. (2.19) using the Wigner-Weisskopf approximation [39, 40] and pick the decay terms from a particular excited state $\left|e^{\prime}\right\rangle$ given by $(\mathcal{L} \rho)_{e^{\prime} e^{\prime}}$ to illustrate this point once more:

$$
(\mathcal{L} \rho)_{e^{\prime} e^{\prime}}=\frac{1}{2} \sum_{e f \eta} \frac{D_{f e^{\prime}}^{\eta} D_{f e}^{\eta} \omega_{e f}^{3}}{3 \pi \epsilon_{0} \hbar c^{3}}\left(\rho_{e e^{\prime}}+\rho_{e^{\prime} e}\right) .
$$

It is important to note that the quantum interference discussed here and elsewhere $[8,9,27-31,41]$ that leads to modifications of the natural line shape is not due to the interference of radiation emitted from excited states $|e\rangle$ and $\left|e^{\prime}\right\rangle$. The interference rather takes place in the different quantum paths leading from a common initial state $|i\rangle$ to a common finial state $|f\rangle$, similar to the mechanisms involved in the phenomena of quantum beats [42]. This interference leads to an enhancement of certain decay paths and a suppression of others while apart from rare exceptional cases, the total decay rate remains constant [41]. The excited states that can contribute interfering paths to the scattering event leading from $|i\rangle$ to $|f\rangle$ are determined by the dipole elements of the excitation and decay as can be seen from Eq. (2.20). Terms with $e \neq e^{\prime}$ in Eq. (2.20) lead to line shape modifications for non-zero $\rho_{e e^{\prime}}$ and $\rho_{e^{\prime} e}$, i.e. coherent excitation of $|e\rangle$ and $\left|e^{\prime}\right\rangle$.

\subsection{Application to hydrogen $2 \mathrm{~S}-4 \mathrm{P}$}

We use the line shape models introduced in the previous section for the description of quantum interference line shifts in precision spectroscopy of the $2 \mathrm{~S}-4 \mathrm{P}$ transition in atomic hydrogen. In this section, we sketch the steps necessary for setting up the two simulations. Using these simulations, a number of properties of quantum interference line shifts are discussed in Sec. 2.4. Since the perturbative approach can not describe a number of effects relevant for our spectroscopy experiment discussed in Sec. 3 (e.g. saturation and optical pumping), the OBE model is used for a comparison with the observed quantum interference line shifts in Sec.6. Additional effects that need to be included in the full description of the experimental line shapes for the particular experimental implementation are listed in Sec. 6.2 after the measurement apparatus has been introduced.

\subsubsection{Relevant energy levels}

Energy levels relevant for the spectroscopy of the $2 \mathrm{~S}-4 \mathrm{P}$ transition in atomic hydrogen are shown in Fig. 2.4. Hydrogen atoms are exclusively prepared in the 
$F=0$ hyperfine sub-level of the meta-stable $2 \mathrm{~S}$ state by optical excitation in the first step of the experiment (for details see Sec.3.1). If the narrowband continuous wave laser at frequency $\nu_{L}{ }^{1}$ driving the $2 \mathrm{~S}-4 \mathrm{P}$ transition is tuned close to resonance with the $2 \mathrm{~S}_{1 / 2}^{F=0} \rightarrow 4 \mathrm{P}_{1 / 2}^{F=1}$ transition $\left(\nu_{1 / 2}\right)$, the $2 \mathrm{~S}_{1 / 2}^{F=0} \rightarrow 4 \mathrm{P}_{3 / 2}^{F=1}$ transition $\left(\nu_{3 / 2}\right)$ plays the role of the perturbing neighboring resonance and vice versa. The natural line width of these two levels is $\Gamma=12.9 \mathrm{MHz}$ and their separation is $\Delta \nu_{F S}^{\text {theo }}(4 \mathrm{P})=1367433.3(3) \mathrm{kHz}[43]$ (see Sec. 7.4.10), i.e. more than one hundred times the natural line width. Transitions from the $2 \mathrm{~S}_{1 / 2}^{F=0}$ to either the $4 \mathrm{P}_{1 / 2}^{F=0}$ and $4 \mathrm{P}_{3 / 2}^{F=2}$ are forbidden by dipole selection rules. The $4 \mathrm{P}$ excitation is followed by a rapid decay via three channels: either to the $1 \mathrm{~S}$ ground state (Ly- $\alpha)$, to the $2 \mathrm{~S}$ state $(\mathrm{Ba}-\beta)$ or a cascade via the $3 \mathrm{~S} / \mathrm{D}$ and $2 \mathrm{P}$ levels to the ground state (Ly- $\alpha)$. The full description of the dynamics involves 52 states, 10 dipole-allowed excitation paths and 306 decay paths. Implementing these for the OBE model leads to a total number of 2707 coupled, complex-valued partial differential equations, which have been solved by numerical integration by Arthur Matveev using high power computation resources provided by the MPG/IPP Rechenzentrum Garching (Sec. 6.2).

Decay constants $\gamma_{i}$ for the individual levels as well as level splittings according to the most recent adjustment of fundamental constants [4] can be obtained from [43]. Hyperfine structure (HFS) splittings are taken from [44, 45].

Out of the emission at different wave lengths, the XUV detectors discussed in Sec. 3.2 are only sensitive to Lyman- $\gamma$ photons at $97 \mathrm{~nm}$ and Lyman- $\alpha$ photons at $121 \mathrm{~nm}$. The three channels above can be treated independently: while the $4 \mathrm{P} \rightarrow 2 \mathrm{~S}$ decay at $486 \mathrm{~nm}$ is not detected, the re-distribution of population in the levels $|i\rangle$ (2S) can have an influence on the observed signals in the other two channels at high laser power. The issue of remaining initial state population detection is addressed in Sec. 2.4.3.

The cascade of subsequent decays leading to the emission of a Lyman- $\alpha$ photon in the last step of the process washes out the contrast of the QI line shifts. We show in Sec. 2.4.3 that in our particular case line shape modifications due to this channel are present, however, they do not influence the apparent line center position.

Both, the spectroscopy signal amplitude as well as the QI line shifts observed in the experiment are dominated by the processes in the Lyman- $\gamma$ channel. If not stated differently, this channel is used in Sec. 2.4 to illustrate basic properties of the QI line shifts. Signals in the Lyman- $\gamma$ channel are calculated using Eqs. (2.12) - (2.14) in the perturbative model. For the full description in the OBE model, Eq. (2.16) is used and signals are separated into the three channels, Lyman- $\alpha$, Lyman- $\gamma$ and Balmer- $\beta$.

\footnotetext{
${ }^{1}$ Measured transition frequencies $\nu_{j}$ are commonly presented in the S.I. unit hertz rather than in radian per second. However, the latter, denoting angular frequencies $\omega_{j}=2 \pi \nu_{j}$, is more common for the theoretical description in the previous sections. For consistency with the following experimental chapters, all frequencies $\nu_{j}$ and line widths $\Gamma_{j}$ are given in hertz from this point on.
} 


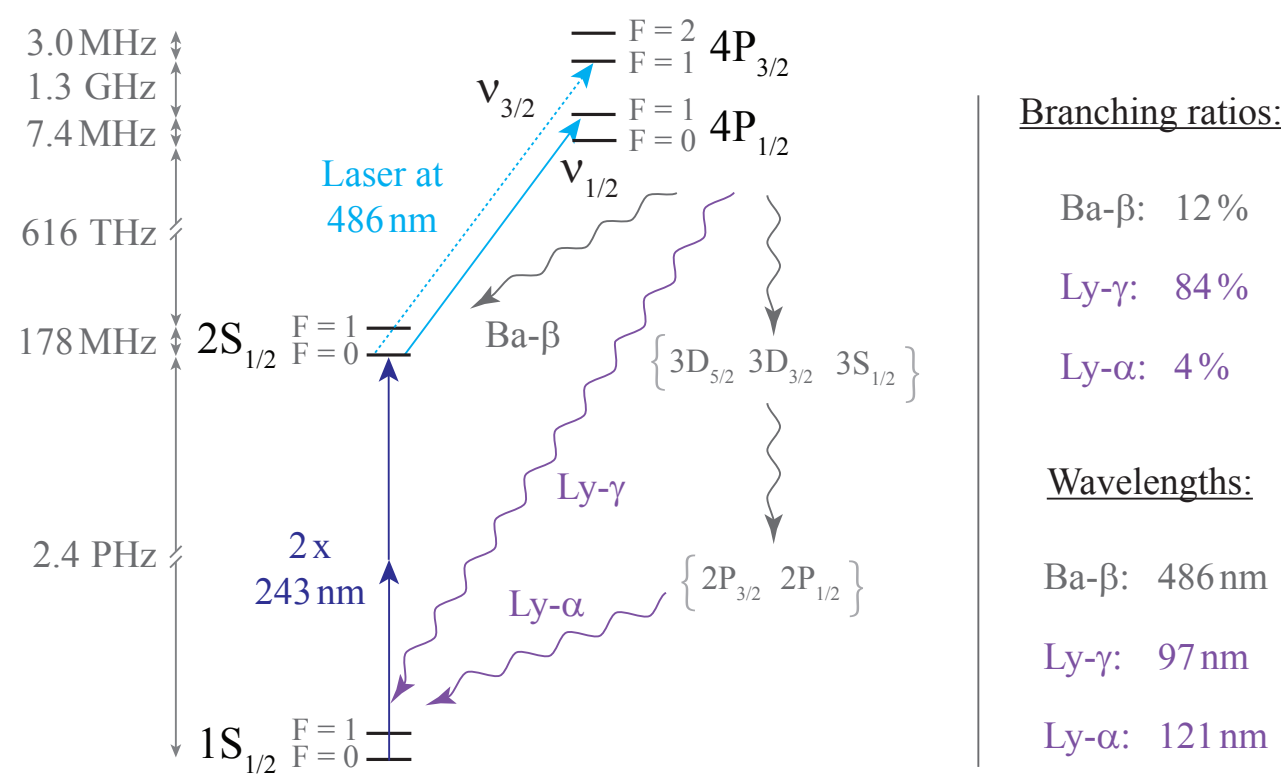

Figure 2.4.: Relevant energy levels for hydrogen $2 \mathrm{~S}-4 \mathrm{P}$ spectroscopy (not to scale). Hydrogen is exclusively prepared in the $2 \mathrm{~S}_{1 / 2}^{F=0}$ meta-stable state (lifetime $\tau_{2 \mathrm{~S}}=1.3 \mathrm{~s}$ ) by Doppler-free two-photon absorption at around $243 \mathrm{~nm}$. Excitation of the $4 \mathrm{P}_{1 / 2}^{F=1}\left(\nu_{1 / 2}\right)$ and $4 \mathrm{P}_{3 / 2}^{F=1}$ state $\left(\nu_{3 / 2}\right)$ states with natural line widths of $\Gamma=12.9 \mathrm{MHz}$ using a second laser at around $486 \mathrm{~nm}$ is followed by a rapid radiative decay: Lyman- $\gamma$ at $97 \mathrm{~nm}(\mathrm{Ly}-\gamma)$, cascades of decays via the 3S, 3D and $2 \mathrm{P}$ levels yielding one Lyman- $\alpha$ photon at $121 \mathrm{~nm}$ $(\mathrm{Ly}-\alpha)$ and Balmer- $\beta$ decay $(\mathrm{Ba}-\beta)$. Excitations from the $2 \mathrm{~S}_{1 / 2}^{F=0}$ to the $4 \mathrm{P}_{1 / 2}^{F=0}$ and $4 \mathrm{P}_{3 / 2}^{F=2}$ levels are forbidden dipole selection rules, i.e. by angular momentum conservation.

\subsubsection{Expanded line shape}

In the case of only two interfering neighboring atomic resonances, the full atomic line shape given by the sum over Eq. (2.12) - (2.14) can be expanded in the same way as illustrated in the discussion of the classical example in Sec. 2.1 [33]. This case applies to our spectroscopy of the $2 \mathrm{~S}-4 \mathrm{P}$ transition in atomic hydrogen and leads to a simple analytic form of the parameters $a_{1}, a_{2}$ and $a_{4}$ in the expanded line shape for the intensities given in Eq. (2.12) - (2.14):

$$
I_{\eta} \propto \frac{a_{1}}{\nu^{2}+(\Gamma / 2)^{2}}+a_{2} \nu+\frac{a_{4} \nu}{\nu^{2}+(\Gamma / 2)^{2}}
$$

Inserting the scattering matrix elements in Eq. (2.9) into Eq. (2.12) - (2.14) and using the dipole matrix elements as described in App. D.1, the parameters $a_{1}, a_{2}$ and $a_{4}$ can be calculated for the $2 \mathrm{~S}-4 \mathrm{P}$ transitions: 


$$
\begin{array}{rlrl}
a_{1}^{\left(4 P_{1 / 2}\right)}=2 & \text { and } & a_{1}^{\left(4 P_{3 / 2}\right)}=\frac{7}{2}-\frac{3}{2} \cos (2 \theta), \\
a_{2}^{\left(4 P_{1 / 2}\right)}=\frac{7-3 \cos (2 \theta)}{\Delta^{3}} & \text { and } & a_{2}^{\left(4 P_{3 / 2}\right)}=-\frac{4}{\Delta^{3}}, \\
a_{4}^{\left(4 P_{1 / 2}\right)}=\frac{1+3 \cos (2 \theta)}{\Delta} & \text { and } & a_{4}^{\left(4 P_{3 / 2}\right)} & =-a_{4}^{\left(4 P_{1 / 2}\right)} .
\end{array}
$$

As has been seen from the classical model, the parameters $a_{2}$ describe the incoherent line pulling, i.e. the fact that the resonance of interest is affected by the far-reaching wing of the perturbing resonance. The parameters $a_{4}$ describe the coherent line pulling due to quantum interference and lead to a dispersive line distortion (see Fig. 2.5). The apparent line shifts due to the latter are given by Eq. (2.6) and are much larger than the incoherent line pulling (Fig. 2.1). Inserting the parameters above into Eq. (2.6), we obtain the apparent shifts (definition analogous to Eq. (2.4)) of the $4 \mathrm{P}_{1 / 2}^{F=1}$ and $4 \mathrm{P}_{3 / 2}^{F=1}$ resonances:

$$
\begin{aligned}
\Delta \nu\left(4 \mathrm{P}_{1 / 2}\right) & =\frac{1+3 \cos (2 \theta)}{4} \frac{\Gamma^{2}}{\Delta} \\
\Delta \nu\left(4 \mathrm{P}_{3 / 2}\right) & =-\frac{1+3 \cos (2 \theta)}{14-6 \cos (2 \theta)} \frac{\Gamma^{2}}{\Delta} .
\end{aligned}
$$

The expanded line shape in Eq. (2.21) is a powerful and analytic tool to study the basic properties of quantum interference line shifts (see Sec. 2.4). It can further by extended to a finite range of detection solid angle as detailed in App. D.

\subsubsection{Comparison of line shape models in the limit of small excitation rates}

In addition to a number of limiting cases discussed in the next section, we test the OBE model used for the description of the experimental data in Sec. 6 by comparison to the perturbative model in the limit of small excitation rates. Fig. 2.5a) shows the calculated signals in the Lyman- $\gamma$ channel using Eqs. (2.12) - (2.14) for the perturbative approach and Eq. (2.16) for the master-equation approach, respectively. For the latter, a single atom at a velocity of $v=100 \mathrm{~m} / \mathrm{s}$ and a excitation light power of $1 \mu \mathrm{W}$ is used, corresponding to an excitation probability of about $1 \%$. At the scale of the full line amplitude, the line shapes are essentially indistinguishable from Lorentz functions due to their large separation. In the perturbative approach, line shapes neglecting the effects of quantum interference are calculated using

$$
I_{-1, \eta}^{\mathrm{NQI}}(\theta) \equiv \frac{1}{2}\left(\cos ^{2}(\theta)+1\right) \sum_{f} \sum_{e}\left|\mathcal{S}_{e, i \rightarrow f}^{-1, \eta}\right|^{2}
$$


instead of Eq. (2.12) - (2.14), i.e. the coherent sum over excited states $\left|\sum_{e} \mathcal{S}_{e, i \rightarrow f}^{-1, \eta}\right|^{2}$ is replaced by an incoherent sum $\sum_{e}\left|\mathcal{S}_{e, i \rightarrow f}^{-1, \eta}\right|^{2}$. The resulting line shapes are simple sums of real valued Lorentz functions.

In the OBE approach, neglecting cross terms is achieved by setting $\Gamma_{i j}=0$ for all $i \neq j$ in Eq. (2.19), i.e. only keeping the terms of normal damping in the Lindblad operator.

The difference in the line shapes with and without the modifications due to quantum interference are shown in Fig. 2.5b) for both models and the emission into the three spherical components $\nu=-1,+1$ and $\nu=0$, corresponding to $\sigma_{-}, \sigma_{+}$and $\pi$ dipole radiation modes, respectively. Note that the units in Fig. 2.5b) are arbitrary, but the same as the units in Fig. 2.5a), i.e. the modifications are on the order of a few parts in $10^{3}$ of the line amplitudes. In the limit of low excitation light power, the residuals calculated using the OBE model coincide with the ones obtained from the perturbative approach. So do the apparent shifts of the resonances discussed in the following sections.

\subsection{Properties of QI line shifts}

The proper description of quantum interference line shifts for an actual experiment can be quite involved. The number of equations to solve for the OBE is $N_{1}^{2}$, where $N_{1}$ is the number of states involved in the dynamics as defined in Eq. (2.17). Furthermore, the experimental geometry has to be considered in detail and properly modeled in the simulations in order to obtain reliable results. This can be extremely difficult, as discussed in App. B. In this section, a number of limiting cases and special arrangements are considered, which can be used for general design considerations of an experiment. Furthermore, the behavior of an existing simulation in these limiting cases may be used for consistency checks.

The polarization state that can be controlled most accurately in an experiment is linear. In addition, rotation of this linear polarization is easy and provides an experimental handle for tests and characterization. Although the equations given in the section above can be applied to arbitrary laser polarizations, we limit the further discussion mostly to the case of linear laser polarization. Furthermore, the major part of our considerations concerns fluorescence light detection, which is the detection scheme used in our experiment. However, it is important to note, that the detection of radiation emitted upon decays populating a certain final state $|f\rangle$ is equivalent to the detection of the population in $|f\rangle$ itself and corresponding arguments apply for either case. Wherever applicable, the arguments are transfered to the case of population detection.

Analogous to our classical example in Sec. 2.1, we define the apparent shift of the resonance $\Delta \nu_{\mathrm{QI}}$ due to the effects of quantum interference as the difference between the true line center at $\nu_{0}$ and the one extracted from fitting a Lorentz function to the spectral feature $\nu_{0}^{\mathrm{LOR}}$. 

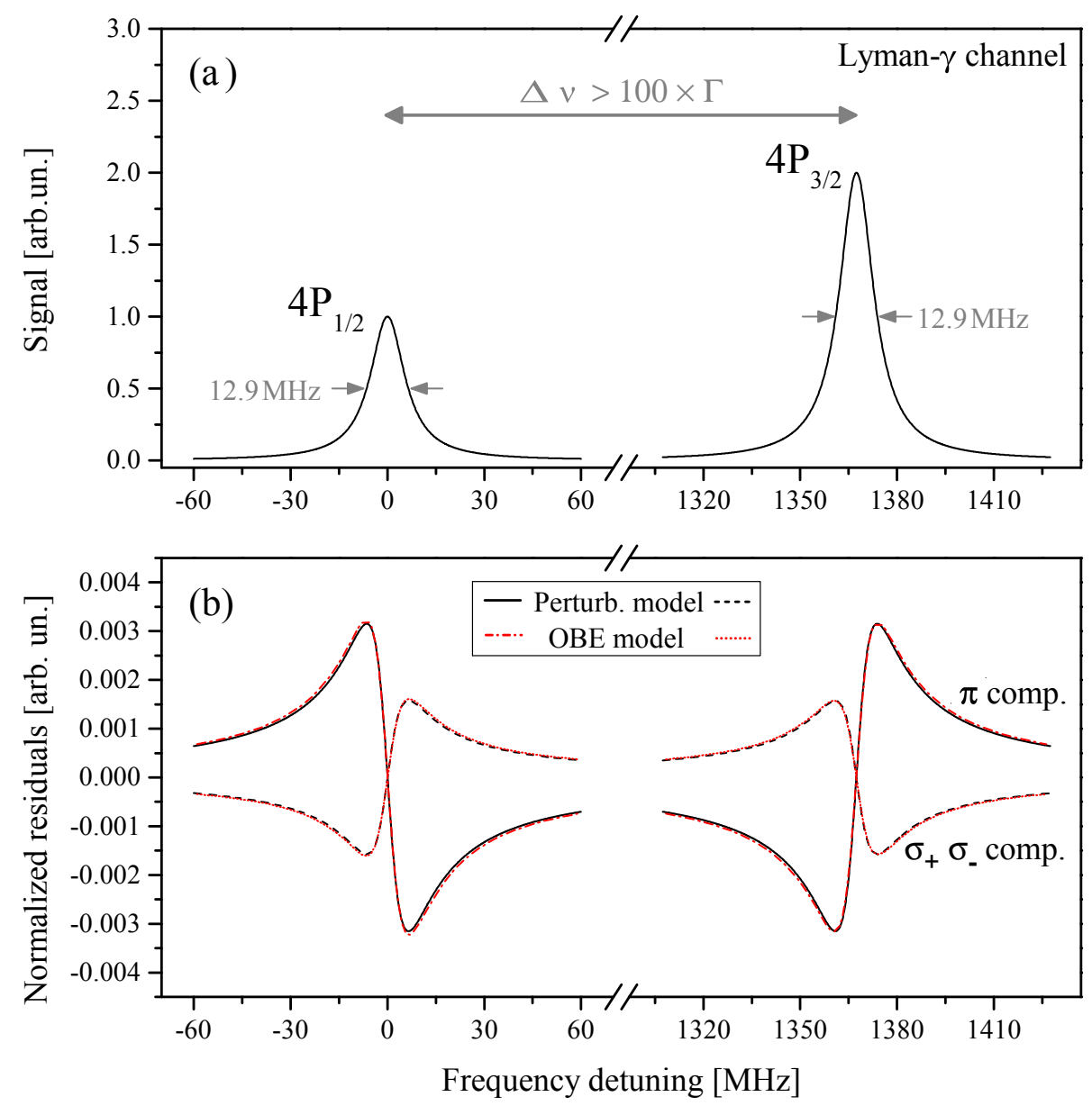

Figure 2.5.: Line shapes of the $2 \mathrm{~S}_{1 / 2}^{F=0}$ to $4 \mathrm{P}_{1 / 2}^{F=1}$ and $4 \mathrm{P}_{3 / 2}^{F=1}$ transitions. (a) Calculated total Lyman- $\gamma(4 \mathrm{P} \rightarrow 1 \mathrm{~S})$ fluorescence (c.f. also Eq. (2.29) for $\left.\theta_{c}=\pi\right)$. The two components are separated by more than 100 times their natural line width of $\Gamma=12.9 \mathrm{MHz}$. (b) Difference (normalized residuals) in the line shapes between the full model including quantum interference terms (Perturb. model: Eqs. (2.12) - (2.14), OBE model: Eq. (2.16)) and modeling by simple sums of real valued Lorentz functions (Perturb. model: Eq. (2.27), OBE model: $\Gamma_{i j}=0$ for all $i \neq j$ in Eq. (2.19)). The line shape distortions due to the quantum interference terms are frequency dependent and asymmetric about the line centers of both components. Note that the units in (b) are arbitrary, but the same as the units in (a), i.e. the modifications are on the order of a few parts in $10^{3}$ of the line amplitudes. Modifications in the line shapes are different for spherical components $\nu=-1,+1$ and $\nu=0$, corresponding to $\sigma_{-}, \sigma_{+}$and $\pi$ dipole radiation modes, respectively. The residuals average to zero if all radiation modes are detected with equal sensitivity. Contributions from $\sigma_{+}$ and $\sigma_{-}$components are identical. In the limit of low excitation light power, the residuals calculated using the OBE model coincide with the ones obtained from the perturbative approach. 


$$
\Delta \nu_{\mathrm{QI}}=\nu_{0}^{\mathrm{LOR}}-\nu_{0}
$$

Note that this apparent shift is a feature of improper modeling rather than a real shift of the line center.

\subsubsection{Spherical components}

The difference in the line shapes with and without the modifications due to quantum interference are shown in Fig. 2.5b) as discussed in Sec. 2.3.3. Modifications in the line shapes are frequency dependent, asymmetric about the line centers of the respective resonances and different for spherical components $\nu=-1,+1$ and $\nu=0$. Contributions from $\sigma_{+}$and $\sigma_{-}(\nu=-1,+1)$ components are identical. The residuals are equal in amplitude but of opposite sign for both transitions. Accordingly, the apparent shifts are of opposite sign and due to the larger line strength of the $2 \mathrm{~S}-4 \mathrm{P}_{3 / 2}$ transition, shifts are smaller than for the $2 \mathrm{~S}-4 \mathrm{P}_{1 / 2}$ transition as can also be seen comparing Eq. (2.25) and Eq. (2.26).

The individual contributions average to zero if all radiation modes are detected with equal sensitivity. The reason for this is the fact that except from rare special cases the quantum interference does not change the overall decay rates of the excited states $|e\rangle$, but it changes the relative strength of different paths emitting into different dipole radiation modes [41] (see also Sec.2.2). Therefore, the effects studied in the following sections and their dependence on specific parameters depend solely on the question whether different decay channels corresponding to different dipole radiation modes are detected with equal probability.

\subsubsection{Finite detection angle, symmetry and periodicity}

The condition of equal detection probability for the different spherical components is not fulfilled when the emitted radiation is only detected in a certain range of emission angles.

The simplest case is the observation of the emitted radiation with an infinitesimally small detector. The intensity incident on the detector can be calculated using Eqs. (2.12) - (2.14). The resulting line shapes are fitted with a Lorentz function to obtain the quantum interference line shifts $\Delta \nu_{\mathrm{QI}}$ or directly by using Eq. (2.25) and Eq. (2.26), which gives the same numbers. Simulations for the $2 \mathrm{~S}-4 \mathrm{P}_{1 / 2}$ transition are used to illustrate a number of features of the QI line shifts.

The line shifts extracted for an infinitesimally small detector along the z-axis as a function of the linear laser polarization angle $\theta_{L}$ are depicted in Fig. 2.6. The line shifts follow a periodic pattern with a period of $180^{\circ}$. As expected, the period is determined by the indistinguishability of linear polarizations rotated by $180^{\circ}$ and is a general feature. The sinusoidal behavior is a feature of the isotropic emission from the $2 \mathrm{~S}-4 \mathrm{P}_{1 / 2}$ state and not expected in general. An example of non-sinusoidal 

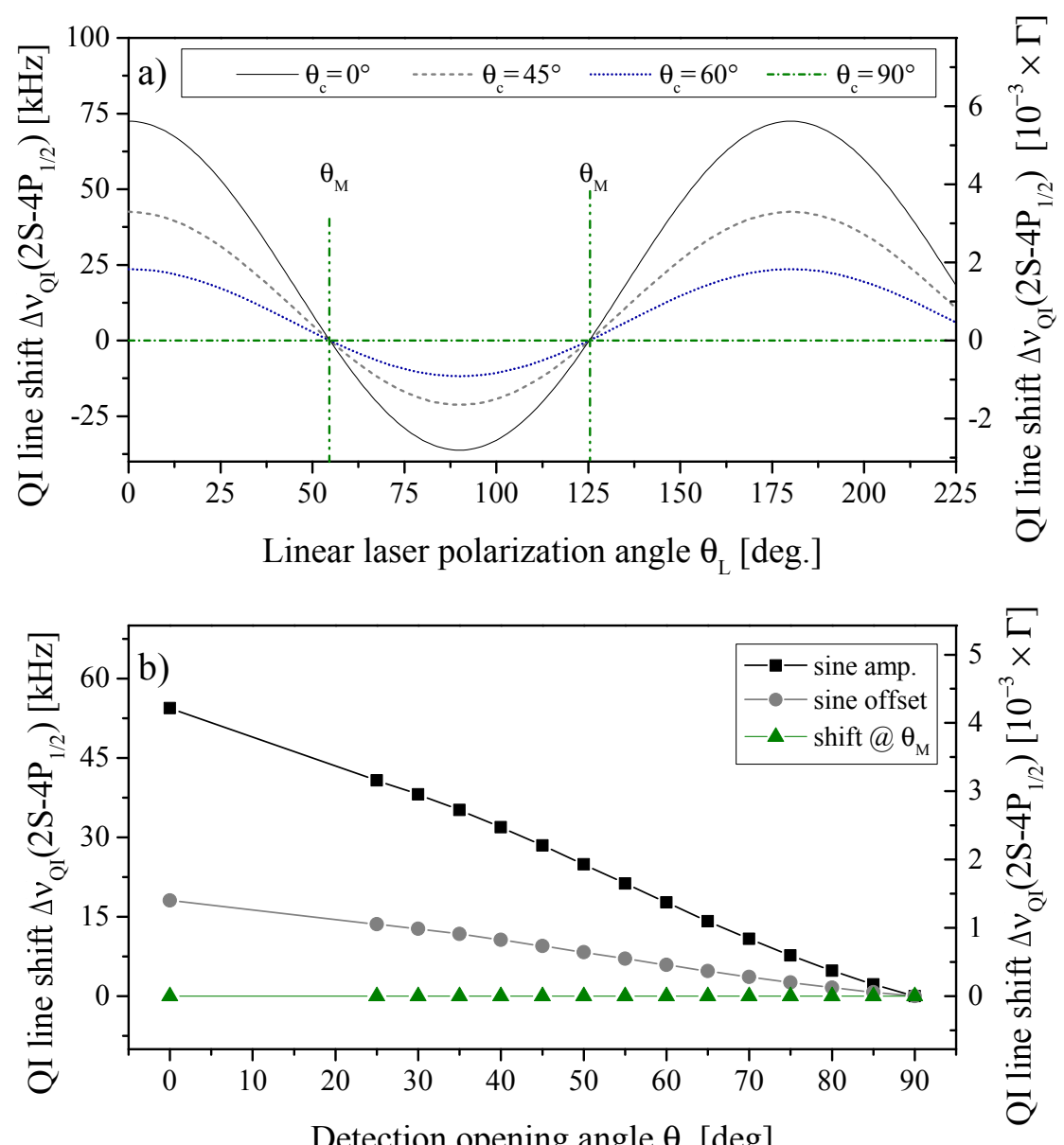

Detection opening angle $\theta_{\mathrm{c}}[\mathrm{deg}]$

Figure 2.6.: Perturbative model: QI line shifts $\Delta \nu_{\mathrm{QI}}$ for the $2 \mathrm{~S}-4 \mathrm{P}_{1 / 2}$ transition. The apparent shift of the resonance $\Delta \nu_{\mathrm{QI}}$ due to the effects of quantum interference is defined as the difference between the true line center at $\nu_{0}$ and $\nu_{0}^{\mathrm{LOR}}$, the line center extracted from fitting a Lorentz function to the asymmetrically distorted resonance profile: $\Delta \nu_{\mathrm{QI}}=\nu_{0}^{\mathrm{LOR}}-\nu_{0}$. (a) QI shifts for different opening angles of a rotationally symmetric detector with opening angle $\theta_{c}$ along the z-axis as a function of linear polarization angle $\theta_{L}$ (see Fig. 2.3). Calculations are performed using the perturbative model (Sec. 2.2.2) for the $2 \mathrm{~S}-4 \mathrm{P}_{1 / 2}$ transition and can be very well approximated by sine functions. Apparent line shifts on the order of tens of $\mathrm{kHz}$, i.e. a few parts in $10^{3}$ of the line width, are predicted. A magic angle $\theta_{M}$ for the polarization direction exists, where residuals from different spherical components cancel and the QI line shift vanishes. (b) Sine amplitude and offsets for QI shifts shown in a). Sine amplitudes decrease with increasing detection opening angle $\theta_{c}$ and the QI line shift vanish in the limit of $\theta_{c} \rightarrow 90^{\circ}$. QI line shifts do not add to zero when averaging over all laser polarizations as can be seen from the since offsets. The sine offsets, however, decrease with increasing $\theta_{c}$ and vanish at $\theta_{c}=90^{\circ}$. QI shifts are zero at $\theta_{M}$, independent of $\theta_{c}$. 
behavior can be found in Fig. 2.8 for the case of the $2 \mathrm{~S}-4 \mathrm{P}_{3 / 2}$ transition. Note that in neither case, the observed QI line shifts average to zero when averaging over all linear polarization anlges $\theta_{L}$ (sine offset, Fig. $2.6 \mathrm{~b}$ ). A similar periodic behavior is observed when the excitation light is circularly polarized and the detector is moved around the emission point.

For most practical cases, the detection system in an experiment will cover a certain range of emission solid angles. We assume a rotationally symmetric detector centered about the z-axis (Fig. 2.3), with opening angle $\theta_{c} \in\left[0^{\circ}, 90^{\circ}\right]$ and equal detection probability for emission from the three dipole radiation modes. Using Eqs. (2.12) - (2.14), the intensity incident on the detector acceptance solid angle can be calculated:

$$
I_{\theta_{c}}=\sum_{\nu, \eta} \int_{0}^{2 \pi} \int_{0}^{\theta_{c}} I_{\nu, \eta}(\theta) \sin (\theta) d \theta d \varphi
$$

QI line shifts as a function of the linear laser polarization angle $\theta_{L}$ for different detection opening angles $\theta_{c}$ are shown in Fig. 2.6 a. Corresponding sine amplitudes, offsets and shifts at the magic angle $\theta_{M}$ are shown in Fig. 2.6 b). The amplitude of the observed QI line shifts decreases with increasing detection opening angle $\theta_{c}$. In the limiting case of $\theta_{c}$ approaching $90^{\circ}$ and $180^{\circ}$, the shifts vanish (see also Sec. 2.4.3).

If the detector features different sensitivity to different spherical components, the individual signal contributions need to be weighted correspondingly. The general case may be rather complex because it depends on the details of the specific detection scheme, which may feature dependences of the detection efficiency on the detection angles $\theta_{d}$ and $\phi_{d}$ as well as potential dependences on the polarization of the emitted light. This point is discussed for the specific detector implementation in the $2 \mathrm{~S}-4 \mathrm{P}$ experiment in Sec. 6.2 and App. B.

\subsubsection{Cancellations in special arrangements}

With the tools and arguments gathered in the sections above, it is possible to identify a number of special arrangements favorable for precision spectroscopy experiments. Certain suggested detections schemes are strictly free from geometry dependent effects in their idealized versions. Others, like the cascade of subsequent decays and the detection of the remaining initial state population for example, may feature large suppression of the line shifts but are not entirely free from the latter.

\section{$4 \pi$ and $2 \pi$ detection solid angle}

Residuals due to quantum interference have been shown in Fig. 2.5. They add to zero if all decay paths are detected with equal probability. One way of achieving this is using a polarization insensitive fluorescence detector with detection opening angle $\theta_{c}=180^{\circ}$, i.e. a detector that covers the full emission solid angle of $\Omega=4 \pi$. Due to the indistinguishably of linear polarizations rotated by $180^{\circ}$, the same argument 
holds for a half sphere, i.e. $\theta_{c}=90^{\circ}$ and $\Omega=2 \pi$ (Fig. 2.6, green dashed-dotted line). Another realization of the same argument is the detection of the total population transfer from all initial states $|i\rangle$ to all final states $|f\rangle$.

\section{Magic angle and tripod detection geometry}

As has been pointed out in Ref. [31], "magic angles" may exist in many cases. At these angles, the line shape modifications add to zero and the corresponding shifts vanish. Examining Eq. (2.24) this magic angle resides at $\theta_{M}=\arccos \left(\frac{1}{\sqrt{3}}\right) \approx 54.73^{\circ}$ (Fig. 2.6). Generally speaking, a magic angle can be found in any orientation of the linear laser polarization with respect to the detection direction that leads to contributions to the line shape from the spherical components that add to zero.

The value of $\theta_{M}$ changes when the detection efficiency is not independent of $\phi$. It is, however, unaffected by a $\theta$ dependence of the detection efficiency as can be argued from the data shown in Fig. 2.6 a) (see also App. D). The quantum interference line shifts vanish for $\theta_{c}=90^{\circ}$ as argued in the previous section and approaches its maximum in the limit $\theta_{c} \rightarrow 0$. The magic angle, however, is the same for all the simulated geometries, i.e. independent of the detection opening angle $\theta_{c}$. At the magic angle, the individual contributions of the different spherical components cancel for all opening angles $\theta_{c, j}$ and the resulting line shape is properly described by a Lorentzian function. Since this is true for all angles $\theta_{c, j}$, the statement also holds for differences in opening angles $\theta_{c, j}-\theta_{c, j-1}$. An arbitrary $\theta$-dependence of the detection efficiency can therefore be constructed by a (in)finite sum of (infinitesimally small) detector segments. It alters the amplitude of the expected QI effect but the magic angle remains unchanged.

Another realization of the argument above is an arrangement of three detectors in a tripod configuration. In addition to the magic angle configuration, this geometry is interesting to consider if multiple access to the interaction point needs to be provided, e.g. for the entrance and exit of an atomic beam and laser beams. An example for such a configuration is the arrangement of detectors along three orthogonal axes for the detection of an isotropically emitting atomic sample. Without loss of generality, one of the axes is placed along the z-axis $(\theta=0)$. Assuming identical detection efficiencies for the three detectors, using Eq. (2.25) and $\theta=90^{\circ}$ for the remaining two detectors, one finds that the shifts exactly cancel, independent of $\theta_{L}$. Any other orientation of the first axis can be accounted for by a re-definition of $\theta_{L}$, so that the cancellation holds for arbitrary orientations of the tripod. Furthermore, the analysis can be extended to detectors with finite solid angle (see App. D.4.3 and D.4.4).

However, the same arrangement does not cancel the apparent shifts in case of the non-isotropic emission from the $4 \mathrm{P}_{3 / 2}$ as can be seen by examining Eq. (2.26) and alternative ways to minimize their influence have to be chosen. 


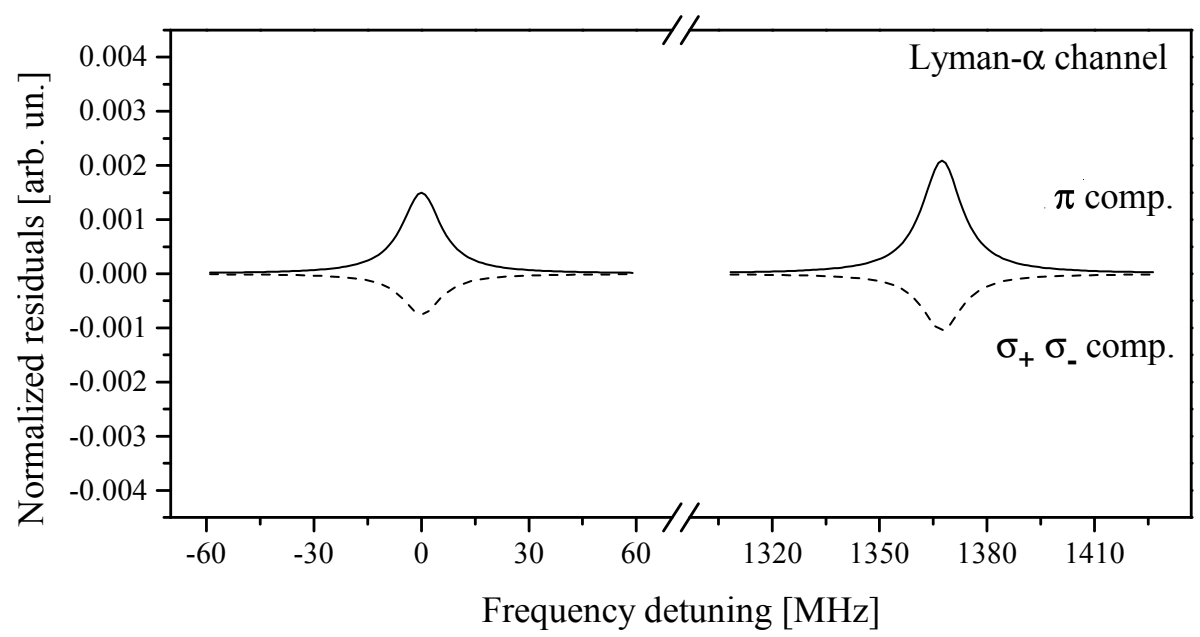

Figure 2.7.: Residuals due to quantum interference for a cascade of subsequent decays (similar to Fig. 2.5). As an example for a cascaded decay, the residuals observed in the Lyman- $\alpha$ channel of the $4 \mathrm{P}$ decay (see Fig. 2.4) are shown. These are calculated using the OBE model, Eq. (2.16), with and without including the terms of quantum interference. This cascade involves three subsequent decays via the $3 \mathrm{~S}$ or $3 \mathrm{D}$ states and the $2 \mathrm{P}$ state and only emission from the last step $(2 \mathrm{P} \rightarrow 1 \mathrm{~S})$ is detected. In contrast to the detection of the first emitted photon, which features the same behavior as seen in the Lyman- $\gamma$ channel (Fig. 2.5), QI residuals are smaller in amplitude and symmetric about the center of the resonances in case of the Lyman- $\alpha$ emission. Note that the units are arbitrary but the same as in Fig. 2.5. QI line shifts due to these symmetric residuals are negligibly small.

\section{Cascades of subsequent decays}

Emission of the fluorescence detected in an experiment may be preceded by a cascade of decays. In this case, the resulting line shape distortions due to quantum interference may be quite different form the ones observed in direct decays. We illustrate this issue by comparing the QI line shape modifications for the Lyman- $\gamma$ channel (direct decay, Fig. 2.5) and the Lyman- $\alpha$ channel (cascaded decay, Fig. 2.7) in hydrogen $2 \mathrm{~S}-4 \mathrm{P}$ spectroscopy (see level scheme in Fig. 2.4). Using the OBE model implemented by Arthur Matveev, the QI residuals in the Lyman- $\alpha$ channel are calculated analogous to the discussion in Sec. 2.3.3. The signal shown in Fig. 2.7 is the last photon emitted in the cascade of subsequent decays. In contrast to the detection of a direct decay (see Lyman- $\gamma$ channel in Fig. 2.5), quantum interference residuals are smaller in amplitude and symmetric about the center of the resonances in case of the Lyman- $\alpha$ channel. Note that the units are arbitrary but the same as in Fig. 2.5. Contributions from $\sigma_{+}$and $\sigma_{-}$components are identical and average to zero with the ones from the $\pi$ components if all radiation modes are detected with equal sensitivity. QI line shifts due to these symmetric residuals are negligibly small.

An intuitive explanation for the different behavior is the following: Quantum interference of different path ways leading from initial states $|i\rangle$ to a final states $|f\rangle$ 
via excited states $|e\rangle$ takes place for coherent excitation of multiple excited states $\left(\left|e_{n}\right\rangle\right.$ and $\left|e_{m}\right\rangle$ (see Fig. 2.2). In the process of spontaneous emission coherence is lost. Therefore the remaining part of the decay cascade from $|f\rangle$ to $\left|f_{\text {cascade }}\right\rangle$ only features normal damping. However, since particular decay channels are enhanced by the effects of quantum interference while others are partially suppressed, the population distribution in $|f\rangle$ is different from the one expected by normal damping. Consequently, the population distributions of states $|g\rangle \ldots\left|f_{\text {cascade }}\right\rangle$ in the subsequent cascade are affected accordingly. This means although the subsequent decay processes only feature normal damping, they are not truly free from the effects of line shape distortions. To which extend an experiment detecting photons or population at a later stage of the cascade is still subject to QI line shifts depends on the complexity and length of the preceding cascade. In the case of hydrogen $2 \mathrm{~S}-4 \mathrm{P}$ spectroscopy, only two preceding steps in the decay cascade are sufficient to suppress the apparent QI line shifts in the Lyman- $\alpha$ channel by about six orders of magnitude compared to the Lyman- $\gamma$ channel due to the resulting symmetric residuals.

\section{Weighted sum of measured transition frequencies}

If the quantum interference is dominated by interference of only two neighboring resonances similar to the case of hydrogen $2 \mathrm{~S}-4 \mathrm{P}$ spectroscopy, the determination of their center of weight position may be used to cancel the accompanying line shifts. To illustrate this point, Eq. (2.25) and Eq. (2.26) are examined and the line shifts depicted in Fig. 2.8. Simple averaging of the extracted line centers reduces the residual amplitude of the QI line shifts, albeit a significant polarization dependence persists (black curve in Fig. 2.8). In contrast to that, weighting the extracted line centers with their $\left(\theta_{L}\right.$ - dependent) line strengths in Eq. (2.22) results in a high degree of cancellation for all anlges $\theta_{L}$ (green curve):

$$
\Delta \nu_{\mathrm{QI}}(\text { centroid })=\frac{3 \sin (\theta)^{2}}{22-6 \cos (2 \theta)} \frac{\Gamma^{4}}{\Delta^{3}} \approx 0
$$

For hydrogen $2 \mathrm{~S}-4 \mathrm{P}$ spectroscopy the ratio $\Gamma / \Delta$ is about $1 / 100$. Therefore the residual shift in the weighted average, i.e. the fine structure centroid, amounts to $\mathcal{O}\left(10^{-6} \Gamma\right)$ and well below $100 \mathrm{~Hz}$. This analysis can also be extended to apply for detectors with finite solid angle coverage (see App. D.4.3 and D.4.4). As a consistency check and demonstration of this scheme for cancellation, it is applied to the observed quantum interference line shifts in $2 \mathrm{~S}-4 \mathrm{P}$ spectroscopy using Voigt fits (Sec.6) in App. A.2.

\section{Remaining initial state population}

It has been argued before that quantum interference takes place between pathways leading from a common initial state to a common final state if multiple excited states are populated coherently. The modification of the decay rates leads to line 


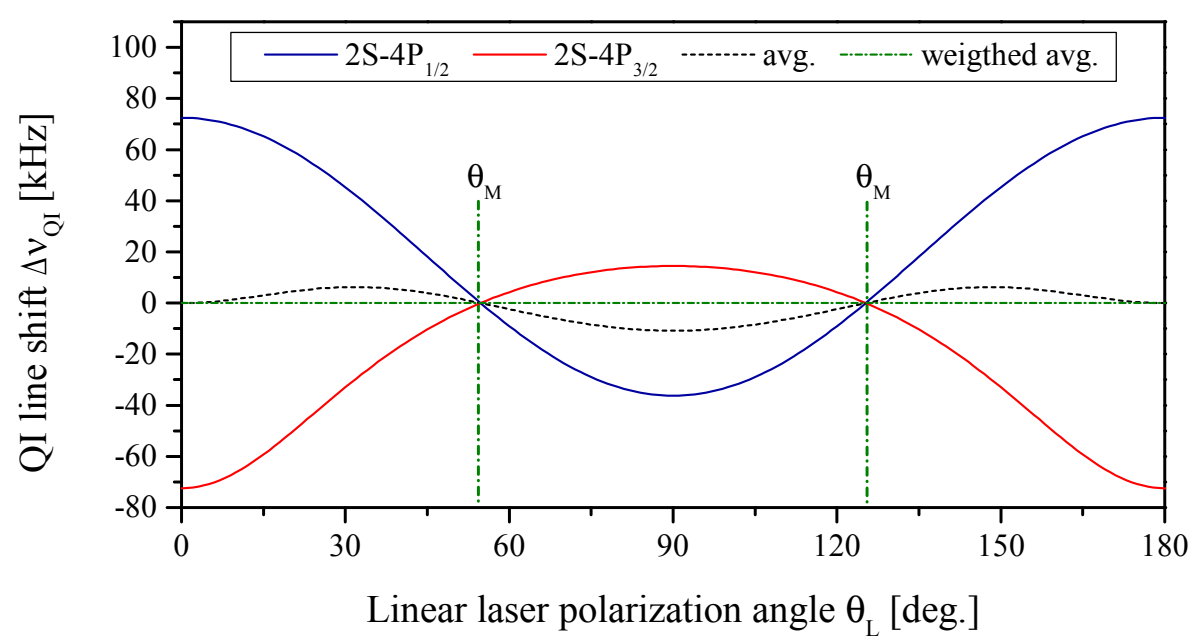

Figure 2.8.: Quantum interference line shifts $\Delta \nu_{\mathrm{QI}}$ for the $2 \mathrm{~S}_{1 / 2}^{F=0} \rightarrow 4 \mathrm{P}_{1 / 2}^{F=1}$ and $2 \mathrm{~S}_{1 / 2}^{F=0} \rightarrow 4 \mathrm{P}_{3 / 2}^{F=1}$ transitions according to Eq. (2.25) and Eq. (2.26). $\theta_{L}$ dependent line shifts vanish at the magic angle of $\theta_{M}=54.73^{\circ}$ for both transitions. The shifts neither add to zero when averaging over all polarization directions in either case nor in a simple average of the transition frequencies (black dashed line). In case of the $2 \mathrm{~S}_{1 / 2}^{F=0} \rightarrow 4 \mathrm{P}_{3 / 2}^{F=1}$ transition the emission is anisotropic and the QI line shifts can not be approximated by a sine function. However, using Eq. (2.22) in a weighted average, the shifts add to zero at any given $\theta_{L}$ (green curve).

distortions and the shifts studied here. On the other hand, this also means that the population remaining in the initial state is unaffected by the quantum interference if there is no decay back to the initial state. An example for such a situation can be constructed in spectroscopy of the dipole-forbidden 2S-3S two photon transition in atomic hydrogen. The direct decay back to the $2 \mathrm{~S}$ state violates angular momentum conservation and is forbidden. Neither does a cascaded decay exist that populates the $2 \mathrm{~S}$ state. For higher lying $n S / D$ states with $n>3$, however, this is no longer the case and quantum interference can affect the observed remaining initial state population as discussed below.

We illustrate the influence of QI line distortions on the observed remaining initial state population using simulations for our $2 \mathrm{~S}-4 \mathrm{P}$ transition as shown in Fig. 2.9. In this case, the decay back to the initial state is non-negligible (branching ratio 12\%, see Fig. 2.4). Calculated signals are shown in Fig. 2.9 a), similar to Fig. 2.5 a). The corresponding residuals brought by quantum interference are shown in part b). Asymmetric residuals are observed in the $2 \mathrm{~S}_{1 / 2}^{F=0}$ state population (initial state) as well as in the $2 \mathrm{~S}\left(F=1, m_{F}= \pm 1\right)$ states which are populated by decays back from the $4 \mathrm{P}$ states. Due to the linear polarization of the excitation light, the $2 \mathrm{~S}(F=1$, $\left.m_{0}= \pm 1\right)$ state can only be populated if an atom scatters at least two photons in sequence. At low excitation light power, the population in this state remains essentially zero. 
The residuals found for the remaining initial state population signal have the same shape as the ones shown in Fig. 2.5 for the detection of Lyman- $\gamma$ fluorescence. However, their amplitudes normalized to the signal amplitude are reduced by the ratio of the corresponding decay rates, i.e. a factor of seven. Correspondingly, detection of the remaining initial state population, i.e. the $2 \mathrm{~S}_{1 / 2}^{F=0}$ state in this case, is much less sensitive to, but not entirely free from, the apparent geometry dependent line shifts.

The conceptional disadvantage for an experimental implementation of this detection scheme is the increased background compared to the fluorescence detection and, as a consequence, a decrease in signal to noise ratio. Detection of the $2 \mathrm{~S}_{1 / 2}^{F=1}$ $\left(m_{F}= \pm 1\right)$ states can in provide better signal to noise ratio than the detection of the $2 \mathrm{~S}_{1 / 2}^{F=1}\left(m_{F}=0\right)$ state because there is no initial population before the interaction. However, there is no advantage regarding QI line shifts, since the shifts are exactly the same as the ones for the Lyman- $\gamma$ fluorescence detection.

The previous hydrogen measurements discussed in Sec. 1.4 almost exclusively used the depletion of the $2 \mathrm{~S}$ population to detect $2 \mathrm{~S}-n \mathrm{~L}$ transitions in a number of different detection geometries. These schemes are generally much less prone to the effects of quantum interference. In the previous experiments, the $2 \mathrm{~S}$ state was populated by electron-impact-excitation, transferring population to all $2 \mathrm{~S}$ Zeeman sub-levels. It has been shown that the population is not necessarily distributed equally among the sub-levels [5]. Both these effects complicate a quantitative study of QI effects in these experiments. Nevertheless, QI line shifts in the most precise two photon experiments may be expected to be smaller than in the case of $2 \mathrm{~S}-4 \mathrm{P}$ spectroscopy (see also [41]). Averaging results from various sources (i.e. geometries) may still be expected to cancel the potential residual shifts due to quantum interference to some extent so that it seems rather unlikely that this effect can explain the observed discrepancy to the $\mathrm{H}$ world data. 

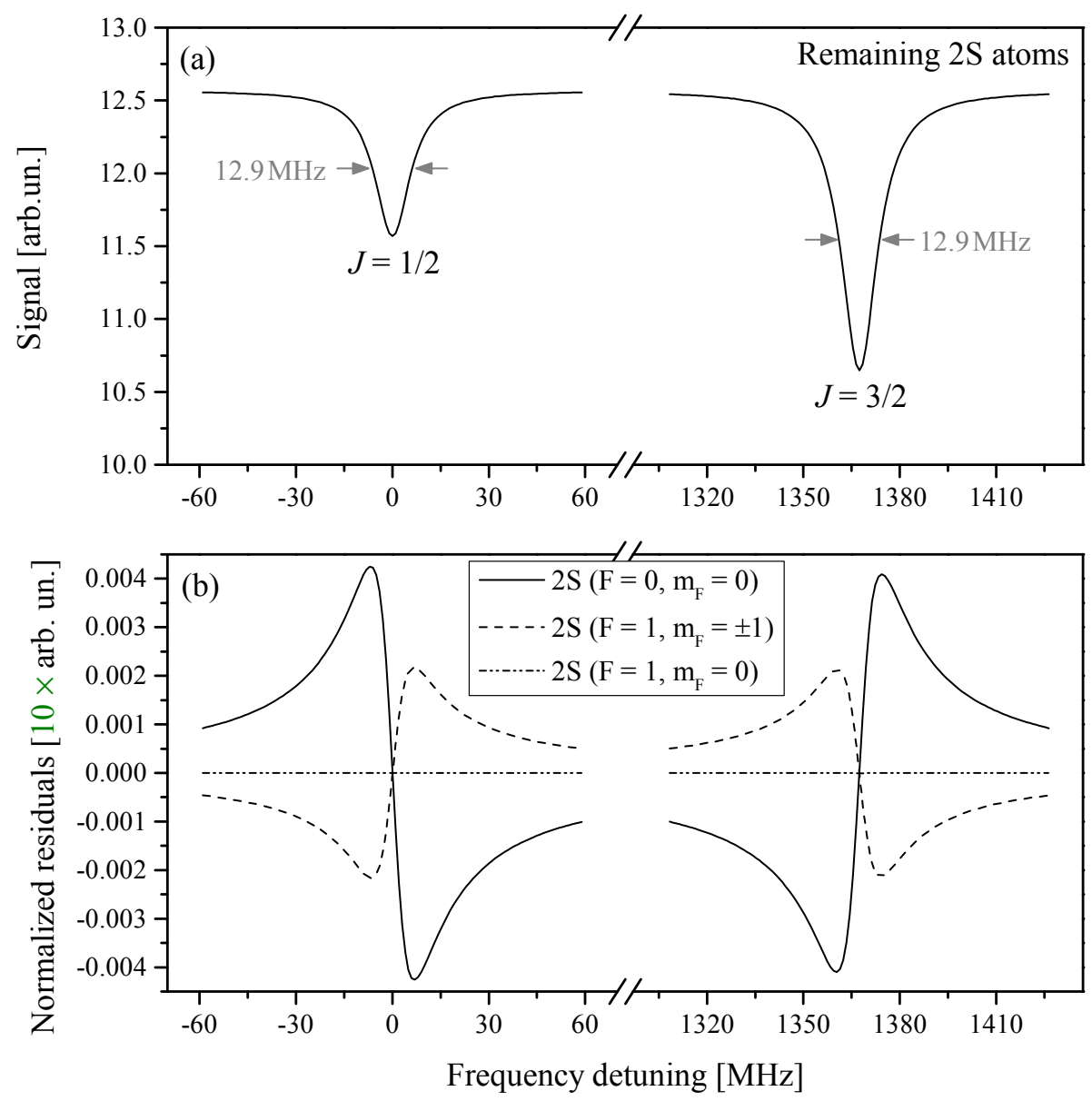

Figure 2.9.: Simulations of QI line distortions for the remaining initial state population (similar to Fig. 2.5) in hydrogen 2S-4P spectroscopy. Line shapes and corresponding residuals are calculated using the OBE approach in the low excitation power limit. Initially, all population is prepared in the $2 \mathrm{~S}_{1 / 2}^{F=0}$ state. The excitation light polarization is linear in this simulation and the $2 \mathrm{~S}_{1 / 2}^{F=1}\left(m_{F}=0\right)$ state can only be populated if an atom scatters at least two photons in sequence. At low excitation light power, the population in this state remains essentially zero. Detection of the remaining initial state population is less prone to the effects of quantum interference line shape distortions. Because only $12 \%$ of the $4 \mathrm{P}$ population decays back to the $2 \mathrm{~S}$ state, the residuals in remaining initial state detection normalized to the line amplitude are much smaller than the ones for the Lyman- $\gamma$ fluorescence in Fig. 2.5. The units in the figures above are arbitrary but identical in both graphs as well as in Fig. 2.5. The suppression is proportional to the ratio of respective decay rates, i.e. 1:7. QI line shifts $\Delta \nu_{\mathrm{QI}}$ are suppressed by the same factor for detection of the remaining $2 \mathrm{~S}_{1 / 2}^{F=0}\left(m_{F}=0\right)$ population. For a state selective detection of the $2 \mathrm{~S}_{1 / 2}^{F=1}$ $\left(m_{F}= \pm 1\right)$ states, which are only populated by decays from the $4 \mathrm{P}$ states, on the other hand, QI line shifts would be identical to those observed in the $\sigma_{+}$and $\sigma_{-}$components of Lyman- $\gamma$ detection. 



\section{Experimental setup}

This section introduces the apparatus that has been developed for the measurement of the $2 \mathrm{~S}-4 \mathrm{P}$ transition during this thesis. A schematic overview is shown in Fig. 3.1, depicting the key components of the experiment detailed in the following sections: Excitation light is provided by two external cavity diode lasers (ECDL) running at $972 \mathrm{~nm}$ [46], stabilized to high finesse reference cavities made from ultra-low expansion glass (ULE, Sec.3.3). Parts of the setup that was used for the measurement of the $1 \mathrm{~S}-2 \mathrm{~S}$ transition frequency $[6,7,47]$ are now used as a cryogenic source of hydrogen $2 \mathrm{~S}$ atoms (Sec. 3.1). The $2 \mathrm{~S}-4 \mathrm{P}$ transition is driven by radiation at around $486 \mathrm{~nm}$ from ECDL2. This light is coupled to an active fiber-based retroreflector (Sec. 3.5) providing large suppression of the first order Doppler effect [25] oriented perpendicular to the atomic beam (2S-4P interaction region) in a separated region. Lyman- $\gamma$ fluorescence photons emitted upon the rapid $4 \mathrm{P} \rightarrow 1 \mathrm{~S}$ decay are detected via photo electrons by channel electron multipliers (CEM 1\&2, Sec. 3.2). Both laser systems are phase-coherently linked to an active hydrogen maser via a fiber frequency comb. The hydrogen maser serves as a frequency reference for the experiment and is calibrated via GPS (Sec. 3.4).

\subsection{Cryogenic beam of hydrogen $2 \mathrm{~S}$ atoms}

A schematic view of the beam apparatus and detector arrangement is shown in Fig. 3.2. Molecular hydrogen is dissociated in a radio frequency discharge running at $30 \mathrm{~W}$ of $2.4 \mathrm{GHz}$ radiation. Hydrogen gas is guided to a T-shaped nozzle via Teflon tubings. The nozzle is attached to a liquid helium flow cryostat and temperature stabilized to $5.8 \mathrm{~K}$. Most of the molecular hydrogen freezes out on the nozzle surface and assists the thermalization of hydrogen atoms. An atomic beam is collimated by diaphragms of $2.1 \mathrm{~mm}$ diameter (front) and $2 \mathrm{~mm}$ edge length (rear). The outermost diaphragms also separate the low vacuum region $\left(\mathrm{P}_{\mathrm{LVR}} \approx 5 \times 10^{-6} \mathrm{mbar}\right)$ containing the nozzle from the high vacuum region $\left(\mathrm{P}_{\mathrm{HVR}} \approx 10^{-8} \mathrm{mbar}\right)$. The latter is pumped from the bottom by a cryopump and accommodates the spectroscopy interaction regions.

A linear enhancement cavity of about $35 \mathrm{~cm}$ length and cavity axis along the atomic beam is formed by two mirrors inside the vacuum chamber ( $\left.w_{0} \approx 290 \mu \mathrm{m}\right)$. During bright phases of the chopper wheel (running at $160 \mathrm{~Hz}$ ), 1S-2S excitation takes place via two photon absorption in the standing wave of the enhancement cavity. The $2 \mathrm{~S}$ hyperfine structure is resolved and atoms are exclusively prepared in the $2 \mathrm{~S}_{1 / 2}^{F=0}$ state. The optical excitation preserves the $1 \mathrm{~S}$ atoms' thermal velocity, 


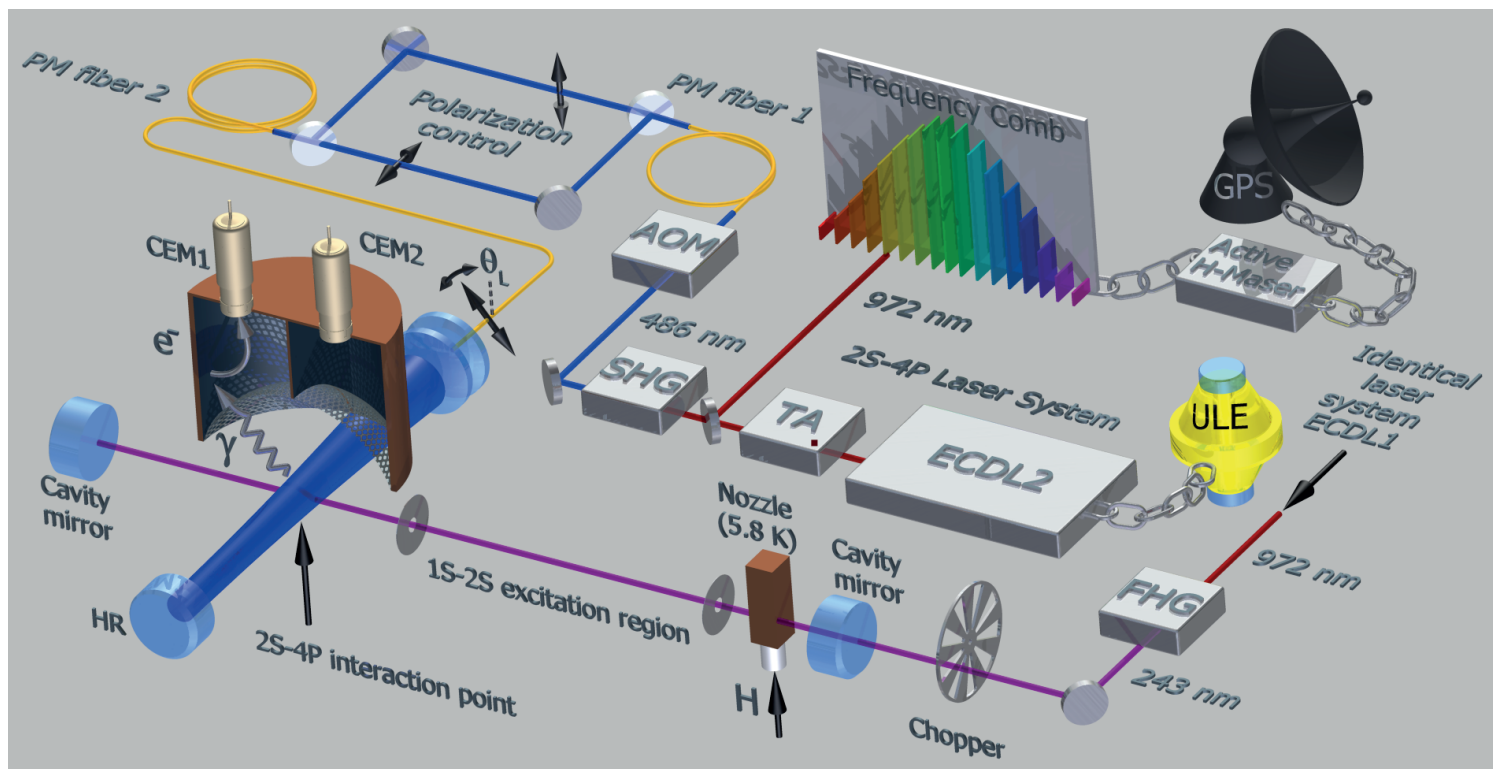

Figure 3.1.: Schematic view of hydrogen $2 \mathrm{~S}-4 \mathrm{P}$ experimental apparatus (not to scale). CEM: channel electron multiplier, AOM: acousto-optic modulator, SHG: second harmonic generation, TA: tapered amplifier, ECDL: external cavity diode laser, ULE: reference cavity made from ultra-low expansion glass, FHG: fourth harmonic generation, GPS: global positioning system. See text for details.

so that the velocity distribution of $2 \mathrm{~S}$ atoms is given by the product of the effusive Maxwellian velocity distribution of the $1 \mathrm{~S}$ atoms at $5.8 \mathrm{~K}$ and the $1 \mathrm{~S}-2 \mathrm{~S}$ excitation probability $[6,47]$. The resulting mean velocity of the $2 \mathrm{~S}$ atoms is about $300 \mathrm{~m} / \mathrm{s}$, ten times less than typically achieved in electron-impact-excitation [5]. The optical excitation also provides a well defined and fast way to discontinue the flux of $2 \mathrm{~S}$ atoms for time-of-flight resolved data acquisition (Sec. 3.2).

Spectroscopy of the 2S-4P transition only takes place in the dark phases of the chopper wheel, i.e. after the $243 \mathrm{~nm}$ light has been blocked and the production of $2 \mathrm{~S}$ atoms has been discontinued. $2 \mathrm{~S}$ atoms travel from the $1 \mathrm{~S}-2 \mathrm{~S}$ excitation region to the field free region around the $2 \mathrm{~S}-4 \mathrm{P}$ interaction point. At this point, radiation at $486 \mathrm{~nm}$ from a second laser propagating perpendicular to the plain of projection drives the transition. The $4 \mathrm{P}$ state rapidly decays down to the $1 \mathrm{~S}$ ground state with a branching ratio of $88 \%$. The corresponding fluorescence is recorded in a time-offlight resolved detection scheme (Sec.3.2). After about $90 \mathrm{~min}$, the frozen gas on the nozzle surface causes significant losses for the enhancement cavity mode and the nozzle needs to be warmed up to $30 \mathrm{~K}$ for about 15 min until the measurement can be continued. 


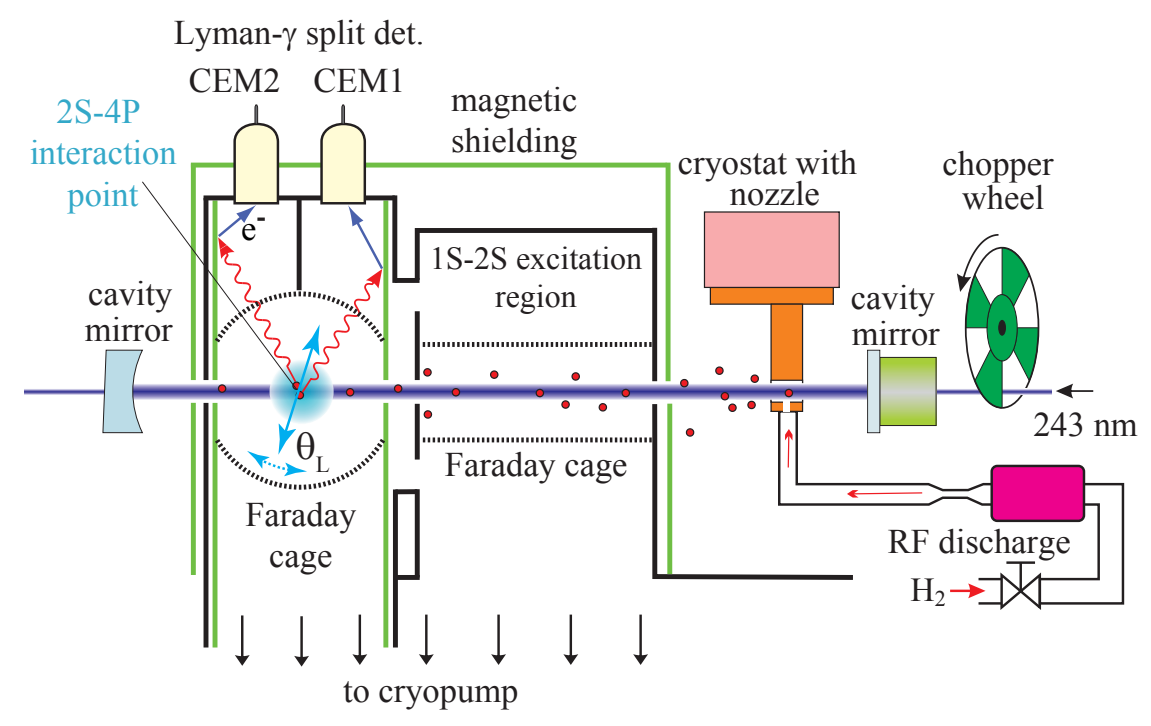

Figure 3.2.: Schematic view of cryogenic hydrogen beam apparatus and XUV fluorescence detectors (not to scale). Atomic hydrogen thermalizes at the T-shaped nozzle that is temperature stabilized to $5.8 \mathrm{~K}$. An atomic beam is formed by the atoms leaving the nozzle to the left and collimated by two diaphragms. Excitation to the meta-stable $2 \mathrm{~S}$ state takes place in the standing wave pattern of a linear enhancement cavity collinear with the atomic beam axis. Only in the dark periods of the chopper wheel, $2 \mathrm{~S}-4 \mathrm{P}$ spectroscopy takes place in the field free region around the interaction point where the transition is driven by $486 \mathrm{~nm}$ laser radiation, propagating perpendicular to the plain of projection (blue shaded area). This allows for a time-of-flight resolved detection of the $4 \mathrm{P}$ fluorescence via channel electron multipliers (CEM 1\&2). The linear polarization of the excitation light at angle $\theta_{L}$ (blue double arrow) can be rotated by $90^{\circ}$ from outside the vacuum chamber (see Fig. 3.2).

\subsection{XUV fluorescence detection}

A large solid angle XUV split detector has been developed for the measurement of quantum interference line distortions in Sec. 6 (Fig. 3.1 and Fig. 3.2) within this work. The detector is split along the direction of excitation light propagation (x-zplane in Fig. 2.3), i.e. perpendicular to the plane of rotation of the linear excitation light polarization $\theta_{L}$ indicated by a black double arrow in Fig. 3.1 (blue double arrow in Fig. 3.2). With this arrangement, the the channel electron multipliers CEM $1 \& 2$ observe the same instantaneous emission from the $2 \mathrm{~S}-4 \mathrm{P}$ interaction point, but cover different ranges in emission solid angle. This arrangement leads to a high common noise suppression, an increased sensitivity to the QI line shifts and the possibility of characterizing the detectors' similarity. The laser polarization angle $\theta_{L}$ can be rapidly altered by $90^{\circ}$ from outside the vacuum chamber as discussed in Sec.3.3. The rotation results in a rotation of the atomic dipole emission patterns by $90^{\circ}$ and is equivalent to exchanging the solid angles covered by the two detectors. This 


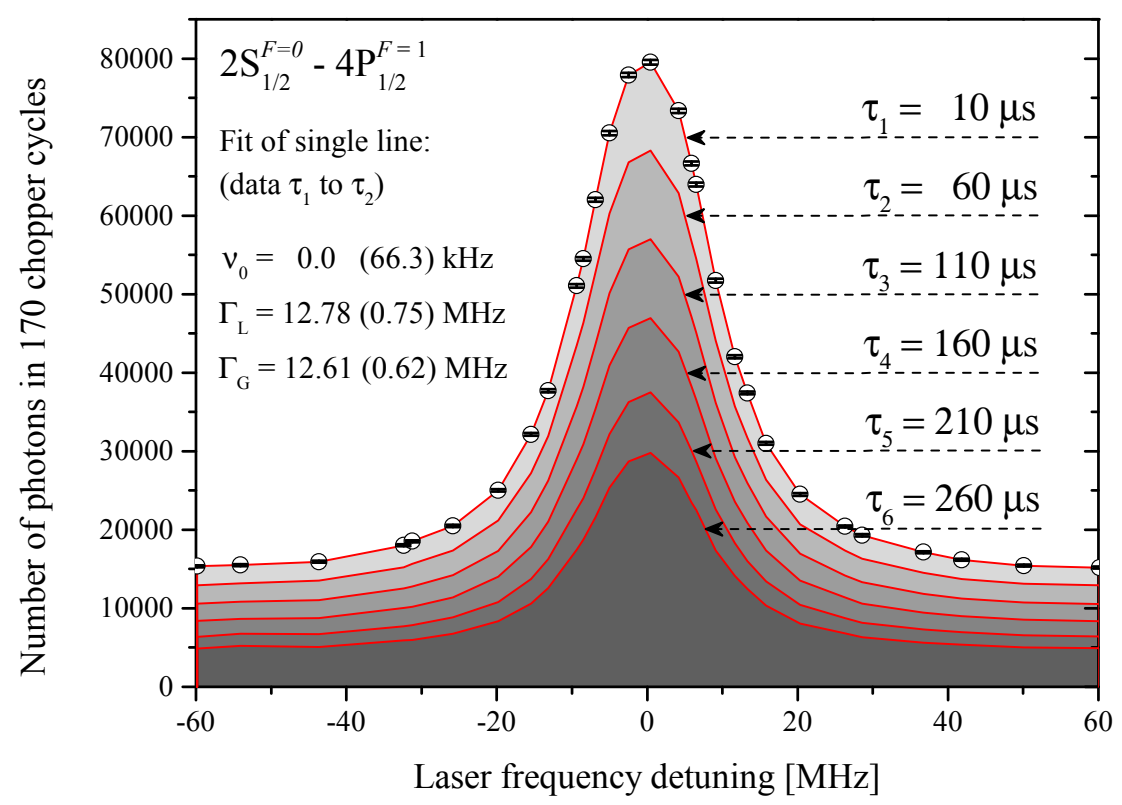

Figure 3.3.: Single line scan sample data for time-of-flight-resolved detection of the $2 \mathrm{~S}_{1 / 2}^{F=0}-4 \mathrm{P}_{1 / 2}^{F=1}$ transition. Independent data sets with different mean velocities are obtained by grouping the fluorescence signal into intervals $\left[\tau_{i}, \tau_{i+1}\right]$, where $\tau$ is the arrival time after the $2 \mathrm{~S}$ excitation has been discontinued. The line scan covers $120 \mathrm{MHz}$ around the line center, i.e. 6 experimental line widths. Statistical uncertainties for the integrated photon number are shown within the open circles.

represents another experimental handle for systematic checks.

A large detection solid angle is achieved by photo electron detection. Lyman- $\alpha$ and Lyman- $\gamma$ photons at $121 \mathrm{~nm}$ and $97 \mathrm{~nm}$ impinge on the graphite coated inner walls of the detector and release photo electrons (Fig. 3.2). The input of CEM 1\&2 is biased at $+270 \mathrm{~V}$, and the electrons can be collected from a emission solid angle that is large compared to one of a typical UV-sensitive photo multiplier tube. A grounded Faraday cage shields the electric fields from the interaction region and its spherical shape provides emission angle independent transmission for the fluorescence photons. Background counts originating from scattered $486 \mathrm{~nm}$ excitation light are entirely suppressed due to the work function of graphite around $5 \mathrm{eV}$. The efficiency of this detector has been modeled (appendix B.2) for the simulation of the observed experimental line shifts due to quantum interference in Sec. 6 .

Resonance curves are obtained by measuring the number of XUV photons as a function of laser frequency, illustrated by the sample data in Fig. 3.3. Photon signals are recorded together with their arrival time $\tau$ using a multichannel scaler after the $243 \mathrm{~nm}$ laser has been switched off $(\tau=0)$. For data evaluation, the recorded signals are grouped into 10 individual delay time intervals $\left[\tau_{i}, \tau_{i+1}\right]$ for each line scan, ranging from delay times of $10 \mu \mathrm{s}$ to $2410 \mu \mathrm{s}$. The width of the intervals was chosen in a way that provides comparable statistics to all 10 data sets and ranges from 50us for early 


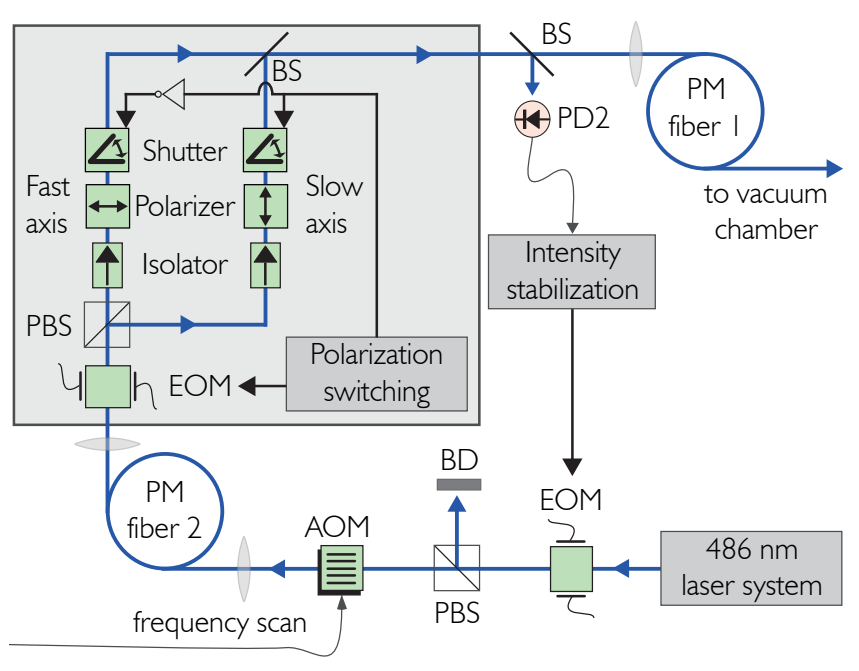

Figure 3.4.: Laser spectroscopy setup. The intensity of the spectroscopy laser at around $486 \mathrm{~nm}$ is stabilized using an electro-optic modulator (EOM) and a polarizing beam splitter (PBS). The frequency of the spectroscopy light is scanned by an acousto-optic modulator (AOM). After the polarization switching unit, that alternately orients the light polarization to coincide with either of the preferred fiber axes, the polarization maintaining (PM) fiber 1 guides the light inside the vacuum chamber. BD: beam dump. PD: photo detector.

delay times depicted in Fig. 3.3 to 1600us (not shown).

The distance from the hydrogen nozzle to the interaction region is $d_{n i}=165 \mathrm{~mm}$ and the maximum velocity $v_{\max }$ for atoms that arrive at a certain delay time $\tau$ is given by the geometry $v_{\max }=\tau / d_{n i}$. The velocity distributions in the individual time windows can be derived from Monte Carlo simulations as well as experimental data $[6,47]$. The corresponding mean velocities range from about $270 \mathrm{~m} / \mathrm{s}$ down to $70 \mathrm{~m} / \mathrm{s}$ and slightly depend on experimental conditions.

\subsection{Laser spectroscopy setup}

Radiation at $486 \mathrm{~nm}$ is generated by a narrow band continuous wave diode laser. It consist of a $24 \mathrm{~cm}$ long external cavity diode laser (ECDL) running at $972 \mathrm{~nm}$, a tapered amplifier (TA) chip and a second harmonic generation stage (SHG). The ECDL is an exact replica of the master oscillator used for $1 \mathrm{~S}-2 \mathrm{~S}$ spectroscopy that has been described before [46]. Stabilizing both lasers to high finesse cavities made from ultra-low expansion glass (ULE) results in narrow laser linewidths smaller than $1 \mathrm{~Hz}$ and fractional frequency drifts of about $2 \times 10^{-16} \mathrm{~s}^{-1}$ at $972 \mathrm{~nm}$ [48]. The spectral purity of the $2 \mathrm{~S}-4 \mathrm{P}$ laser is tested by a beat note with the $1 \mathrm{~S}-2 \mathrm{~S}$ laser at $486 \mathrm{~nm}$.

The intensity of the $2 \mathrm{~S}-4 \mathrm{P}$ laser is stabilized by a combination of an electro-optic modulator (EOM) and a polarizing beam splitter (PBS) as shown in Fig. 3.4. The laser frequency can be scanned via an acousto-optic modulator (AOM). A polarization maintaining fiber (PM fiber 2) guides the light to the polarization switching part of the setup. The light is split up into two arms. Each arm features an optical isolator, a high quality Glan-Thompson polarizer (extinction 1 part in $10^{5}$ ) and a mechanical shutter. After recombining the two path ways on a polarization insensitive beam splitter, the light is coupled into another PM fiber (PM fiber 1) guiding 
the light into the vacuum chamber. The transmission direction of the polarizers have carefully be aligned to coincide with the preferred fiber axes (slow and fast axis) of the PM fiber. Alternately opening one of the mechanical shutters results in a rotation of the laser polarization by $90^{\circ}$ inside the vacuum chamber. The intensity extinction ratio has been measured to be better than 200 at the $2 \mathrm{~S}-4 \mathrm{P}$ interaction point for both fiber axes. The PM fiber output that defines $\theta_{L}$ inside the vacuum chamber is fixed to a rotatable mount that is used to alter $\theta_{L}$ in the measurement of quantum interference line shifts in Sec. 6.

\subsection{Laser frequency calibration}

Both laser systems ECDL1 and ECDL2 are phase-coherently linked to an erbiumdoped fiber frequency comb which is referenced to an active hydrogen maser. The maser serves as the frequency reference for the experiment and is calibrated via the global positioning system GPS, resulting in a fractional frequency uncertainty of well below 1 part in $10^{13}$ at any given time. The absolute frequencies of the laser systems are determined via a beat note with the frequency comb at $972 \mathrm{~nm}$. The absolute frequency of ECDL2 at $486 \mathrm{~nm}$ at a given time $t$ can thereby be determined via:

$$
\nu_{\mathrm{ECDL} 2}^{486}=2 \times\left(N \nu_{\mathrm{rep}}+2 \nu_{\mathrm{CEO}}-\nu_{\mathrm{LO}}\right)+2 \times\left(\nu_{\text {beat }}(t)-\nu_{\mathrm{AOM}}(t)\right),
$$

where $\nu_{\text {rep }}=250 \mathrm{MHz}$ denotes the repetition rate of the the frequency comb, $\nu_{\mathrm{CEO}}=30 \mathrm{MHz}$ the carrier-envelope-offset frequency, $\nu_{\mathrm{LO}}=150 \mathrm{MHz}$ (only for the measurement of $\nu_{1 / 2}$ ) the frequency of an additional local oscillator used to mix down the frequency of the beat note to $\nu_{\text {beat }}(t) \approx 10 \mathrm{MHz}$ and $\nu_{\mathrm{AOM}}(t) \approx 350 \pm 30 \mathrm{MHz}$ the frequency of the AOM used for scanning over the atomic resonance. The comb mode numbers are $N\left(\nu_{1 / 2}\right)=1233042$ and $N\left(\nu_{3 / 2}\right)=1233044$, respectively.

\subsection{Suppression of the first order Doppler effect}

${ }^{1}$ The first order Doppler shift is the leading order systematic effect for the measurement of the 2S-4P absolute transition frequency detailed in Sec. 7. This section summarizes the cancellation of the shift using an active fiber-based retroreflector (AFR) developed within this thesis. After introducing the principle of two beam excitation for one photon transitions, common techniques involving corner cube retroreflectors and optical cavities are discussed and the most important advantages of the AFR are summarized.

\footnotetext{
${ }^{1}$ The content of this section has been published in [25] and was only slightly modified to fit the general structure of this thesis.
} 


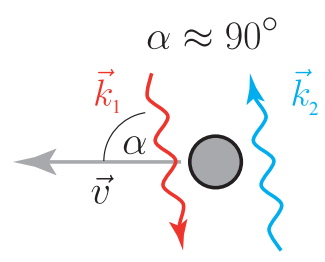

$\Delta \nu=2 v \cos (\alpha) / \lambda$

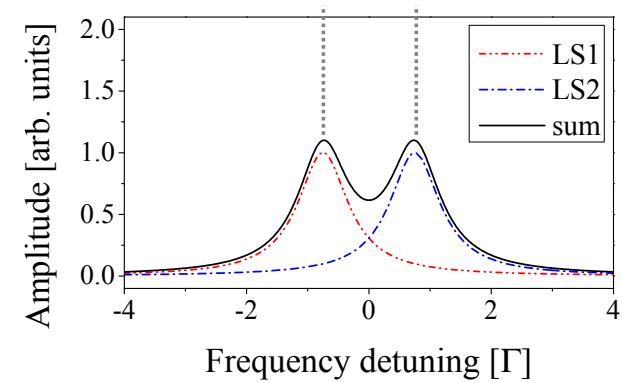

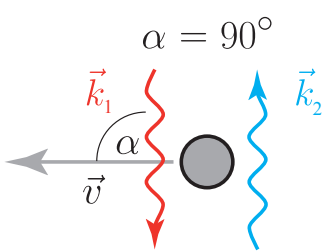

$\Delta \nu=0$

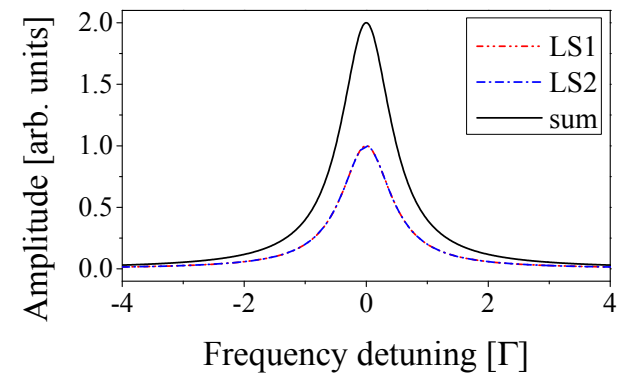

Figure 3.5.: Cancellation of the first order Doppler effect by utilizing counter-propagating laser beams. Left: counter-propagating laser beams at an angle different from $\alpha=90^{\circ}$ with respect to the atomic trajectory. The observed spectrum is a doublet with a separation $\Delta \nu=2 v \cos (\alpha) / \lambda$. The individual line shapes (LS1 and LS2) resulting from the interaction with one of the laser beams are indicated by dashed lines, the observed spectrum by a black solid line. The frequency detuning is measured in units of the natural line width $\Gamma$. Right: For an atom crossing the lasers at $\alpha=90^{\circ}$ the two components overlap. In either case the line center is unaffected provided that both laser beams have the same intensity. In practice one has to deal with curved wavefronts. Also in this case the average local first order Doppler effect vanishes provided that the wave fronts retrace each other, i.e. wave vectors $\overrightarrow{k_{1}}$ and $\overrightarrow{k_{2}}$ are anti-parallel at any given point.

\subsubsection{Principle of two beam excitation}

The first order Doppler shift of an individual atom moving at a velocity $\vec{v}$ in a plane wave described by a wave vector $\vec{k}=2 \pi / \lambda \hat{e}_{k}$ is given by:

$$
\Delta \nu_{D}=\frac{1}{2 \pi} \vec{k} \cdot \vec{v}=\frac{v}{\lambda} \cos (\alpha)
$$

where $\lambda$ denotes the wavelength, $v$ the magnitude of the atom's velocity vector and $\alpha$ its angle with respect to the wave vector. Eq. (3.2) expresses the first oder Doppler shift in terms of wavefronts that the atom traverses per unit of time. It also suggests that instead of reducing the atomic velocity the first order Doppler effect may be canceled by arranging an atomic beam perpendicular to the laser beam. However, the requirements on alignment for a modern precision experiment are difficult to achieve in this way [49], in particular when the divergence of the atomic beam and the laser beam wave front curvature have to be taken into account.

A standard procedure to reduce this difficulty has been to employ two counterpropagating laser beams with $\overrightarrow{k_{1}}+\overrightarrow{k_{2}}=0$, where $\overrightarrow{k_{1}}$ and $\overrightarrow{k_{2}}$ denote the respective 
wave vectors. This has been effective for several established Doppler-free methods like saturation spectroscopy [50] and two-photon spectroscopy [6]. In the latter case the Doppler shifts add to zero when an atom absorbs one photon from each of the two waves. For perfectly counter-propagating plane waves this compensation holds everywhere, independent of velocity. Neither a first order Doppler shift nor Doppler broadening takes place in this ideal situation so that the dominating effect becomes the much smaller second order Doppler effect [6] which will not be discussed here. In addition to the Doppler-free component, the two-photon transition amplitude comes with another component that is Doppler broadened [50]. This one is due to the absorption of two photons from either of the two waves.

Driving a one-photon dipole-allowed transition in a perfect standing wave of two exactly counter-propagating waves of equal intensity shows very similar features. In that case one obtains a doublet of symmetrically Doppler shifted spectral components, one for $\overrightarrow{k_{1}} \cdot \vec{v}$ (line shape LS1 in Fig. 3.5), the other for $\overrightarrow{k_{2}} \cdot \vec{v}=-\overrightarrow{k_{1}} \cdot \vec{v}$ (line shape LS2 in Fig. 3.5). The center of weight of these components is free of the Doppler shift while the Doppler broadening manifests itself in terms of the separation of the two lines as sketched in Fig. 3.5.

\subsubsection{Corner cubes and optical cavities}

The common technique to generate anti-parallel waves is to reflect a laser beam using a corner cube retroreflector (see for instance [5, 31]). Two types are commercially available: prism type corner cubes and hollow retroreflectors. The latter can be manufactured with a specified maximum angular deviation of $\varepsilon=3 \mu \mathrm{rad}$ [51], where $\varepsilon$ is the angle between the incoming and the outcoming beams. Using Eq. (3.2), the residual uncompensated Doppler shift at angles $\alpha$ close to 90 degree $(\alpha=\pi / 2+\delta \alpha)$ measured with respect to $\overrightarrow{k_{1}}$, can be written as:

$$
\Delta \nu_{\text {resid }}=\frac{1}{2} \frac{v}{\lambda}[\sin (\delta \alpha)-\sin (\delta \alpha-\varepsilon)] \approx \frac{v}{2 \lambda} \varepsilon .
$$

In the last step, we have expanded the expression for small $\delta \alpha$ and $\epsilon(\delta \alpha, \epsilon \ll 1 \mathrm{rad})$. It is important to note, that the right hand side of Eq. (3.3) is independent of $\delta \alpha$ in first order, i.e. if no other imperfections are present, the residual Doppler effect is independent of the angle between the laser beams and the atomic beam. The small angular deviation $\epsilon$ rather leads to a constant offset, as is also depicted in Fig. C.4 in the appendix.

Reflecting the light off the three orthogonal surfaces of a corner cube retroreflector leads to wavefronts that are anti-parallel with high precision $(\epsilon=3 \mu \mathrm{rad})$ and according to Eq. (3.3) would correspond to a residual uncompensated Doppler shift of 2 parts in $10^{6}$ of the full collinear shift. However, the retroreflected beam is displaced laterally and its polarization is rotated. As a result atoms that cross the two beams in sequence might be optically pumped into a particular Zeeman sublevel in the first interaction zone and are then probed with a different polarization in the second 
zone. In addition the polarization rotation significantly complicates the analysis of polarization dependent line distortions due to quantum interference (see Sec. 6 and $[31,52])$. Illuminating the edges of the corner cube leads to wave front distortions [5], complicated polarization behavior [53] and attenuations that add to possible bulk and Fresnel losses. The latter reduces the amplitude of the spectral component that corresponds to the reflected beam. All of these effects give rise to asymmetric line distortions and hence to systematic shifts of the observed line center.

In order to circumvent these problems the two counter-propagating laser beams must have anti-parallel wave fronts such that $\overrightarrow{k_{1}}+\overrightarrow{k_{2}}$ vanishes everywhere. For a reflected Gaussian beam this means that its waist must be localized at the retroreflector and the latter must be at $90^{\circ}$ relative to the laser beam axis. In addition the local intensities of the two waves must be identical. For this the retroreflector should be highly reflective and wavefront distortions should be minimized. Corner cube retroreflectors may be used to achieve this goal when the laser beam is split into two arms. One contains the corner cube reflector to detect misalignments and the other one is used for precision spectroscopy [54].

An alternative to the use of a corner cube is the so-called "cat's eye" configuration (see for instance Fig. 1 in [55]). Such a device may have good performance in terms of parallelism of the counter-propagating waves. Beam deviations of a few microradian may be possible using a 2f-configuration and lenses with focal lengths of tens of centimeters. However, the overlap of the two waves is not ensured and the additional lens introduces Fresnel and bulk losses. Even if the polarization is maintained, a partial overlapping of the beams can efficiently unbalance the fluorescence (sequentially) produced by each wave, similar to the case discussed for corner cubes above.

For deep-UV applications, where our AFR system may not readily be applied due to the lack of high quality optical single mode fibers, utilizing a Sagnac interferometer may represent an alternative in some cases [56]. However, it is important to note that small imbalances in the counter-propagating waves (i.e. imperfections of the beam splitter in the Sagnac interferometer) give rise to significant residual Doppler shifts as we discuss further below (see Eq. (C.5) in App. C.1).

Another possibility that works in principle for all spectral regions from the IR to the deep UV would be to use a high finesse linear cavity which has the advantage of enhancing the resonant, i.e. phase retracing mode, while at the same time attenuating possible mismatched waves. However, limiting the intra-cavity power $P_{i c}$ to minimize excitation light power dependent systematic effects, such as the ac-Stark effect, optical pumping and saturation, means that an even lower power has to be coupled into the cavity and/or the finesse has to be restricted. Using some reasonable numbers for our 2S-4P experiment detailed above $\left(P_{i c}<15 \mu \mathrm{W}\right.$ and beam waist $w_{0} \sim 2 \mathrm{~mm}$ ), one finds that it will be difficult to obtain a high quality error signal to stabilize a cavity with such a low power level. A second laser that is far detuned from resonance with the spectroscopy transition can be used to stabilize the cavity. Such a setup, however, would significantly increase the complexity of the system. 


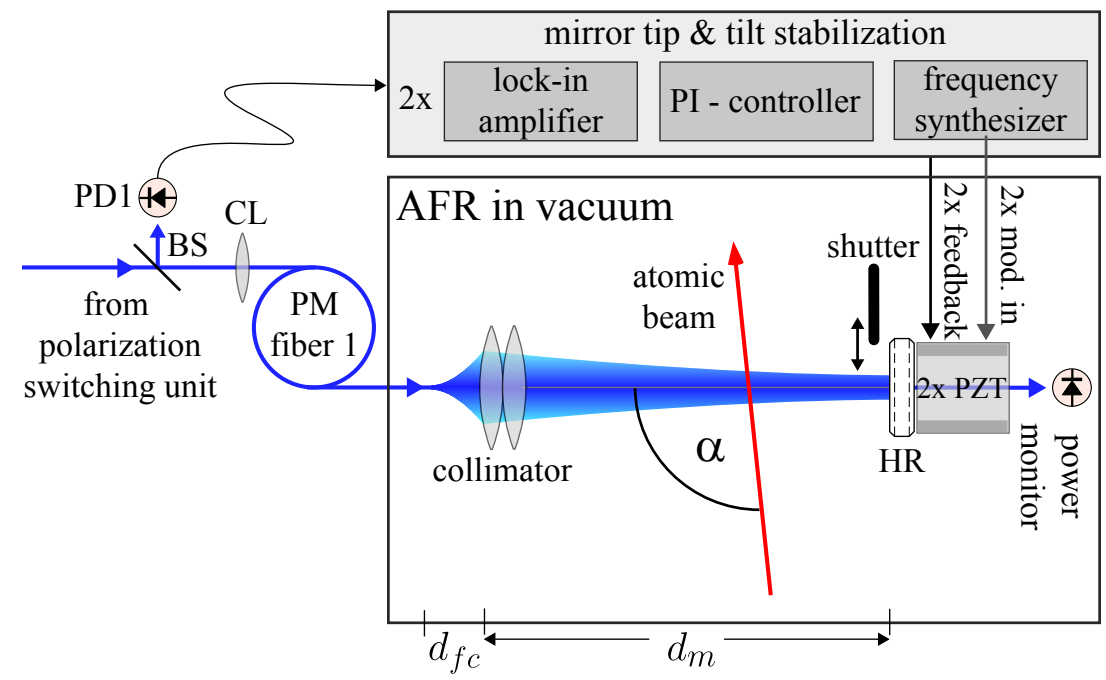

Figure 3.6.: Schematic view of the active fiber-based retroreflector (AFR). The main components are a polarization maintaining single mode optical fiber (PM fiber 1), a collimator with low imaging aberrations $(f=27 \mathrm{~mm})$ and an actively stabilized high reflectivity mirror (HR, $R=99.995 \%$ ). The light emerging from fiber 1 is collimated such that the waist $\left(w_{0}=2.1 \mathrm{~mm}\right)$ is located on the flat HR mirror at a distance of $d_{m}=260 \mathrm{~mm}$. For an ideal Gaussian beam, this configuration leads to exactly retracing wave fronts. Deviations from this ideal situation may be due to spherical aberrations of the collimating lens. Experimentally this is verified by using an atomic beam with a variable mean velocity (see Sec. 4). Another polarization maintaining single mode fiber (PM fiber 2, Fig. 3.4) is used as mode cleaner to improve reproducibility of the coupling efficiency to PM fiber 1 . The latter may be affected by the settings of the acousto-optic modulator (AOM), the electrooptic modulator (EOM) and the laser operating conditions (Fig. 3.4). PD: photo-detector, PZT: piezo-electric transducer, BS: beam splitter, CL: coupling lens.

\subsubsection{Active fiber-based retroreflector concept}

In order to enable a measurement of the absolute frequency of the $2 \mathrm{~S}-4 \mathrm{P}$ transition in atomic hydrogen with an improved accuracy [49, 52, 57], an active fiberbased retroreflector (AFR) as shown in Fig. 3.6 has been developed. Light from a narrow-band continuous wave laser is coupled into a polarization maintaining single mode optical fiber (PM fiber 1 [58]). At the output of the fiber, inside the vacuum chamber, the light is collimated with an objective of low imaging aberrations and a focal length of $f=27 \mathrm{~mm}$ [59]. The output of a step index fiber is very close to a Gaussian mode [60]. The objective position is chosen such that the waist of the collimated beam is located on the flat mirror (HR) with near unity reflectivity ( $R=99.995 \%$ [61]) that reflects the light back into the fiber. The fiber then serves as a small aperture converting angular misalignment of the returning beam into a 
a)

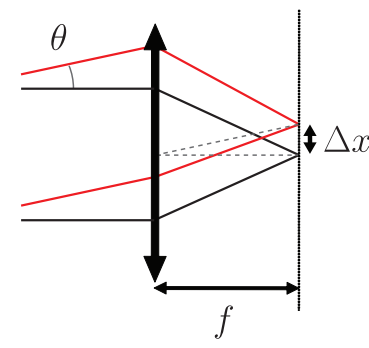

b)

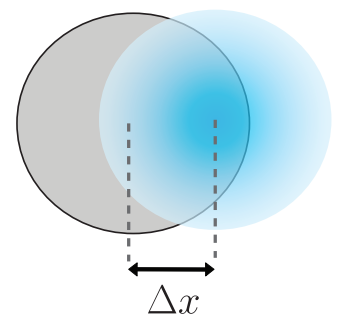

c)

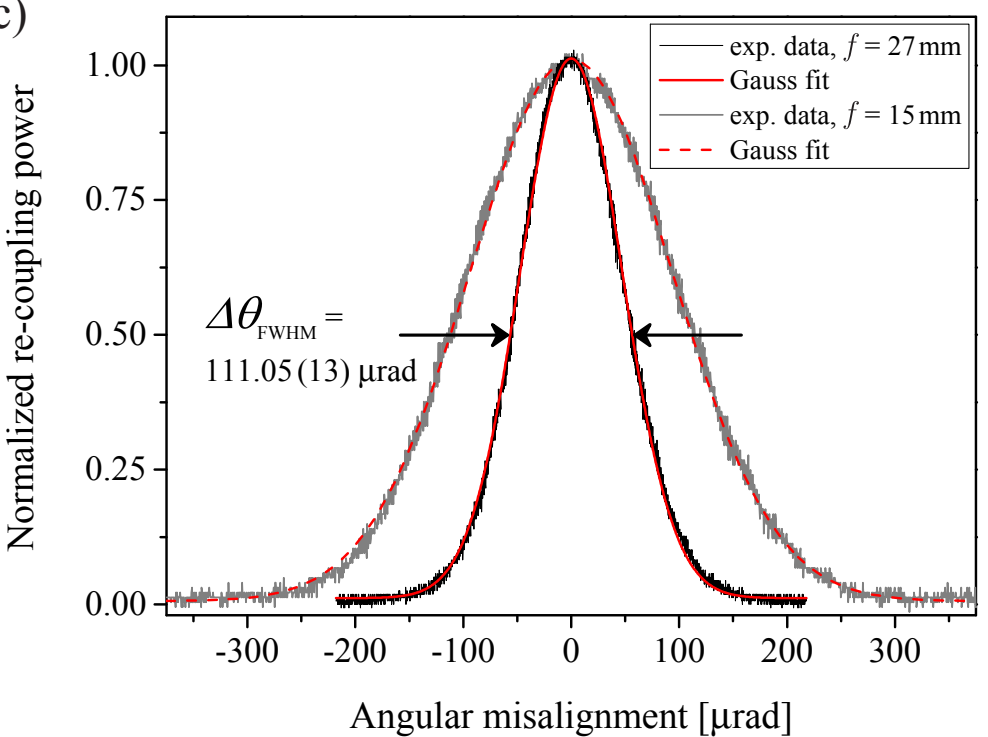

Figure 3.7.: Estimation of the alignment sensitivity of the active fiber-based retroreflector (AFR) shown in Fig. 3.6. a) Misalignment of the returning beam by an angle $\theta$ leads to misplacement of the focus by $\Delta x \approx \theta f$ and hence to a reduced re-coupling efficiency back into the single mode fiber. The convolution of the returning mode with the fiber mode as shown in b) gives $\exp \left(-\Delta x^{2} / w_{0}^{2}\right)$ with a full width at half maximum of $\Delta \theta_{\text {FwнM }}=2 w_{0} \sqrt{\ln (2)} / f$. For $f=27 \mathrm{~mm}$ and $w_{0}=1.90(25) \mu \mathrm{m}$ as specified [58] we obtain $\Delta \theta_{\text {FwHM }}=117(15) \mu \mathrm{rad}$. c) Intensity measured at PD1 (Fig. 3.6) as a function of the returning beam angular misalignment (tilt). The misalignment is inferred from the applied PZT voltage and has been calibrated by measuring the beam deflection angle as a function of PZT voltage at a distance of $5 \mathrm{~m}$. The response curve follows a Gaussian as expected and has a full width at half maximum of 111.05 (13) prad, in good agreement with the estimation using a) and b). For comparison the response curve of another collimator with $f=15 \mathrm{~mm}$ is shown (light gray). The reduced sensitivity to misalignment and the larger half width at half maximum of 222.1 (3) prad corresponds well to the reduction in focal distance.

measurable amplitude signal at photo detector PD1. Modulation of the HR mirror tip and tilt angles allows to detect and correct misalignments using lock-in amplifiers (appendix C.2).

A simple estimate for the sensitivity to angular misalignment can be obtained using paraxial ray optics as illustrated in Fig. 3.7a. Light incident on a thin lens under different angles is focused to different locations in the focal plane. Small angular misalignments result in a lateral displacement of the focused Gaussian beam with respect to the fiber mode. For the experimental implementation the measured full width at half maximum angle of the fiber re-coupling efficiency is found to be $\Delta \theta_{\mathrm{FWHM}}=111.05$ (13) prad. The sensitivity can be increased by using collimation optics with larger focal lengths (Fig 3.7). 
A detailed study of possible imperfections of the AFR system in given in appendix C.1. These include residual imaging aberrations of the collimating objective and misalignments in the collimator position $d_{f c}$ that both lead to phase fronts that do not re-trace each other. Furthermore, the influence of imbalances in the intensities of the phase-retracing beams is studied. The active stabilization scheme used in the experiment is detailed in appendix C.2. The theoretical considerations in Sec. C.1 give useful estimates for the performance of the AFR system. To throughly quantify the potential residual uncompensated Doppler shift, however, a direct measurement is required. The performance of the AFR is characterized in Sec. 4 and the residual uncompensated Doppler shift measured utilizing the time-of-flight resolved detection introduced in Sec. 3.2. 


\section{Measurement of the residual uncompensated Doppler shift}

1 To compare the situation with and without the Doppler suppression of the AFR, the returning laser beam can be periodically blocked by a shutter depicted in Fig 3.6. The atomic resonance is scanned in random (frequency) order, and at each frequency point, two sets of data are acquired. For one set the shutter is open, i.e. the AFR is active, and for the second one the shutter is closed and only one beam interacts with the atomic sample. This type of differential measurement offers a high degree of common noise suppression for other systematic effects that vary on a time scale longer than the data acquisition time of about 3 seconds per laser frequency setting. The fiber, collimation optics and HR mirror of the AFR are fixed to a rotatable mount inside the vacuum chamber (not shown in Fig. 3.6, see App. E). The angle $\alpha$ between the laser beams and the atomic beam can be adjusted remotely by rotating the AFR axis with respect to the atomic beam. The angle $\alpha$ can be read from a vernier scale inside the vacuum chamber.

We rewrite the Doppler shift in Eq. (3.2) using the parameter $\eta(\delta \alpha)$ that measures the slope of the Doppler shift at an angle $\alpha=90^{\circ} \pm \delta \alpha$ as a function of atom velocity $v$ in $\mathrm{Hz} /(\mathrm{m} / \mathrm{s})$ or simply $\mathrm{m}^{-1}$ :

$$
\Delta \nu_{D}=\eta(\delta \alpha) \times v
$$

where

$$
\eta(\delta \alpha)=\frac{1}{\lambda} \times \sin (\delta \alpha)
$$

The position of $\alpha=90^{\circ}$ is found by measuring resonances with one laser beam only (shutter closed, similar to red data in Fig. 4.1) at different angle settings. The position of $\alpha=90^{\circ}$ on the vernier scale is found by interpolation to the point of zero slope $\left(\eta=0 \mathrm{~m}^{-1}\right)$ with an uncertainty smaller than $0.01^{\circ}$ after about 30 minutes of data acquisition. Alternatively, albeit with reduced sensitivity, the alignment may be determined by measuring the observed line width as a function of the laser-toatomic-beam angle [5]. The angle $\alpha=90^{\circ}$ can be set with an uncertainty of $0.08^{\circ}$ and is limited by the read-off accuracy of a vernier scale.

500 pairs of $2 \mathrm{~S}-4 \mathrm{P}$ resonances (shutter open and closed) have been recorded for this demonstration in about 4 hours of data acquisition time. For small deviations from $\alpha=90^{\circ}$, the atomic spectra can be fit with Voigt functions to measure the

\footnotetext{
${ }^{1}$ The content of this chapter has been published in [25] and was only slightly modified to fit the general structure of this thesis.
} 


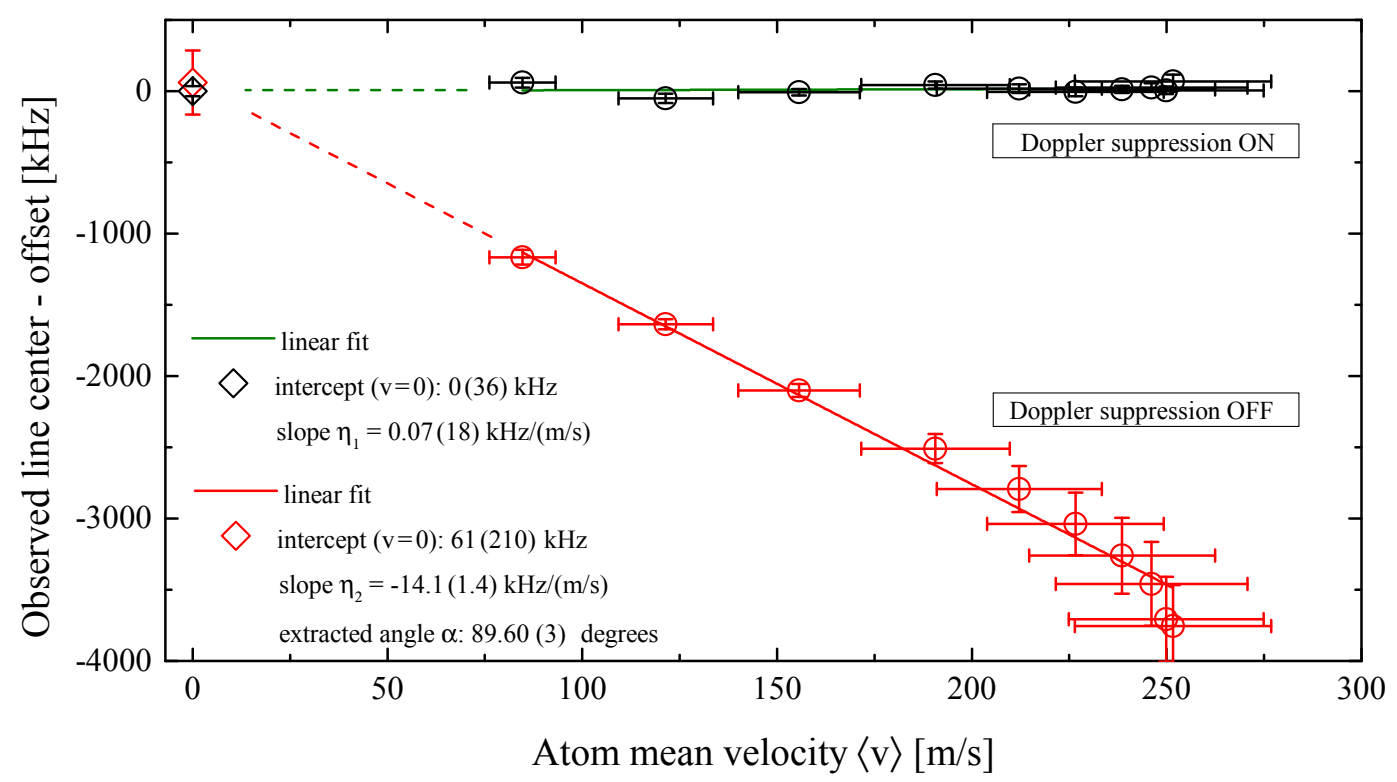

Figure 4.1.: Observed transition frequency in the lab frame with and without Doppler suppression. Imperfections of our AFR are characterized by a differential measurement of the 2S-4P transition at an angle of $\alpha=89.60^{\circ} \pm 0.03^{\circ}$ ( $\alpha$ as defined in Fig. 3.6, 4 hours of data acquisition). Red circles: Doppler suppression off, i.e. the shutter in Fig. 3.6 is closed. Black circles: Doppler suppression active, i.e. shutter open. Experimental uncertainties for the latter case are small on this scale and shown inside the open circles. For better readability, the frequency axis is offset by $\approx 616 \mathrm{THz}$ such that the linear fit of the black data extrapolates to zero at $v=0$. The observed slope $\eta_{1}$ (Doppler suppression on) of the extracted transition frequency as a function of atom mean velocity $\langle v\rangle$ is limited by statistics and compatible with zero. Due to its large sensitivity to the Doppler shift $\left(\eta_{2}=\right.$ $14.1(1.4) \mathrm{kHz} /(\mathrm{m} / \mathrm{s}))$, the data with the Doppler cancellation switched off is a suitable tool for the determination of $\alpha$, however not for precision spectroscopy at an accuracy level of a few tens of kilohertz, i.e. a few parts in $10^{5}$ of the full collinear Doppler shift. Still, intercepts at $v=0$ of the two data sets coincide within the given uncertainties.

uncompensated Doppler shift. Mean values of the extracted line centers are shown in Fig. 4.1 as a function of the mean velocity $\langle v\rangle$ of atoms contributing to the corresponding delayed signals. The observed slopes $\eta$ are limited by statistics and compatible with zero if the AFR is active. The angle $\alpha_{\exp }=89.60^{\circ} \pm 0.03^{\circ}$ extracted from the experimental data using only one laser beam (red circles) is compatible with the less accurate mechanical reading of the vernier scale and shows that the adjustment is reproducible with sufficient accuracy. Due to its large sensitivity to the Doppler shift, the data with the Doppler cancellation switched off is a suitable tool for the determination of $\alpha$, however not for precision spectroscopy at an accuracy level of a few tens of $\mathrm{kHz}$, i.e. a few parts in $10^{5}$ of the full collinear Doppler shift. Still, the intercepts at $v=0$ of the two data sets agree, indicating internal consistency 


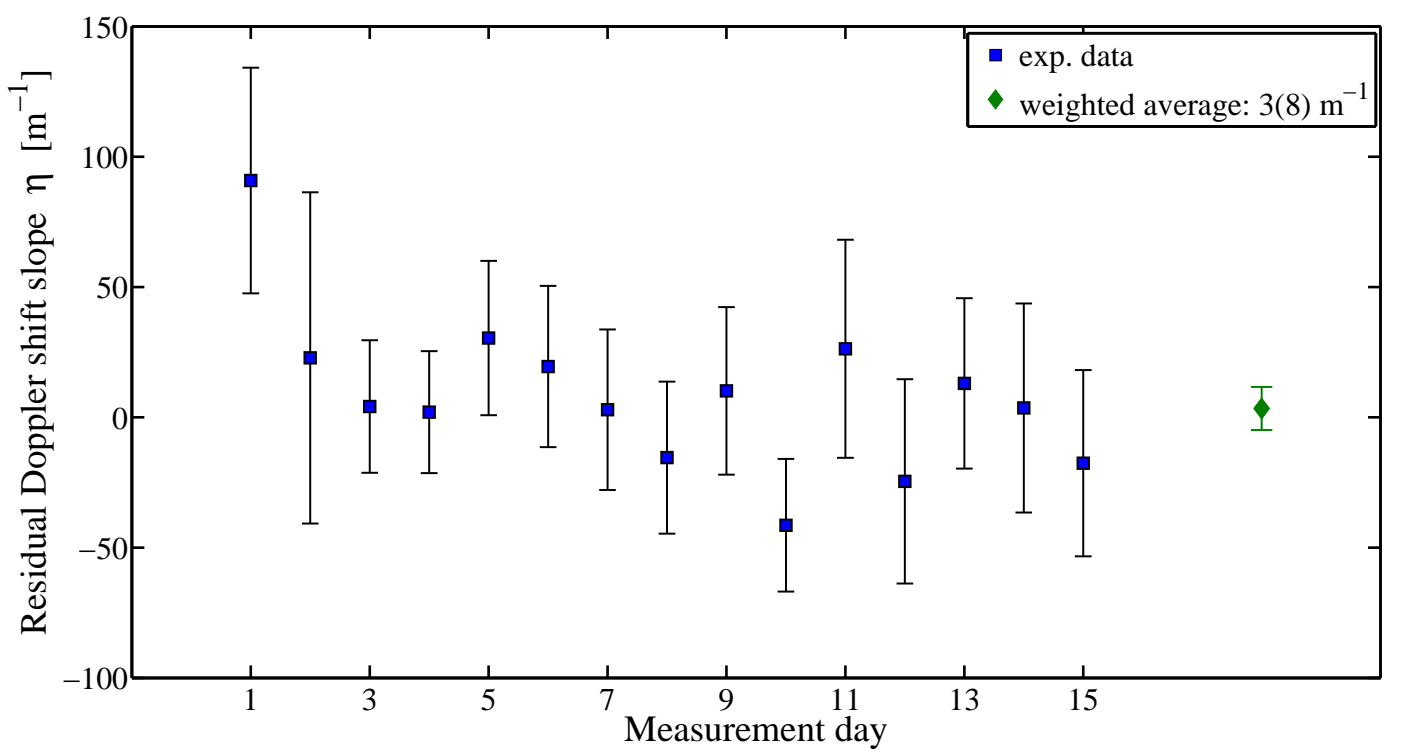

Figure 4.2.: Characterization of residual uncompensated first order Doppler shift. The angle between the atomic beam and the counter-propagating laser beams provided by the active fiber-based retroreflector (AFR) is adjusted to $\alpha=90^{\circ} \pm 0.08^{\circ}$. Line centers of the $2 \mathrm{~S}-4 \mathrm{P}$ transition in atomic hydrogen have been extracted from a total number of 48,000 resonance profiles as a function of the mean velocity of the atoms contributing to the respective signal. Blue data points indicate average values of the residual uncompensated Doppler shift slope $\eta_{j}$ per measurement day and active AFR (similar to black data in Fig. 4.1). Average uncertainties per day amount to about $35 \mathrm{~m}^{-1}$. No excessive scatter of the experimental data is observed and we determine the overall average to be $\eta_{\exp }=3(8) \mathrm{m}^{-1}$. This limit corresponds to a reduction of the full collinear Doppler shift $\eta\left(90^{\circ}\right)=2.05 \times 10^{6} \mathrm{~m}^{-1}$ to less than 4 parts in $10^{6}$.

of the analysis.

According to Eq.( 3.2) and Eq. (3.3) the Doppler shift for blocking and unblocking the back reflected laser beam is given by $\delta \alpha \times v / \lambda$ and $\epsilon \times v / 2 \lambda$, respectively. Typically the deviation $\delta \alpha$ from perfect perpendicular alignment between the atomic and the laser beams is much larger than the angle between the forward and backward traveling laser beam $\epsilon$. A comparison between the two situations does not give a reliable estimate for $\epsilon$. Unfortunately this is the angle that is required to estimate the residual first order Doppler shift. Therefore we directly measure the residual uncompensated Doppler shift in a long term measurement.

In 15 days of measurement, distributed over one month of run time, a total number of 48,000 individual resonances of the $2 \mathrm{~S}-4 \mathrm{P}$ transition have been acquired. The data set has been acquired with the AFR suppressing the Doppler shift, similar to the black data shown in Fig. 4.1. The angle between the atomic beam and the laser beams has been adjusted to $\alpha=90^{\circ} \pm 0.08^{\circ}$ using the same procedure described 
above. As before, we use our time-of-flight resolved detection scheme to obtain $2 \mathrm{~S}-4 \mathrm{P}$ line centers as a function of the mean velocity $\langle v\rangle$ of atoms contributing to the signal. Average values of the residual uncompensated Doppler shift slopes $\eta_{j}$ are shown in Fig. 4.2 for each measurement day. Uncertainties for the mean values per measurement day are approximately $35 \mathrm{~Hz} /(\mathrm{m} / \mathrm{s})$, or simply $35 \mathrm{~m}^{-1}$. For comparison, the expected slope using only one laser beam at an angle of $89.92^{\circ}$ is $2871 \mathrm{~m}^{-1}$. No excessive scatter of the experimental data is observed $\left(\chi^{2} / d o f=0.72\right.$, $d o f=14$ ) and we determine the overall average to be $3 \pm 8 \mathrm{~m}^{-1}$. This measurement of the uncompensated Doppler shift is compatible with zero and includes all possible imperfections of the Doppler compensation using our AFR as discussed above and in App. C. Compared to the full Doppler shift of $\eta\left(90^{\circ}\right)=2.05 \times 10^{6} \mathrm{~m}^{-1}$, this limit corresponds to a reduction to less than 4 parts in $10^{6}$.

Assuming a residual uncompensated Doppler shift of the observed size was solely caused by an angular mismatch of the returning beam of an otherwise ideal AFR, the corresponding misalignment amounts to $\epsilon_{\mathrm{AFR}}=2.9(7.7)$ prad. Note that this value corresponds to the overall average over more than four weeks of data taking. While it does not necessarily mean that possible angular deviations are smaller than this value at any given time, opening the vacuum chamber every second day of measurement for maintenance, readjustments in the apparatus and changing of experimental conditions (in particular laser powers and polarization) neither lead to excessive scatter nor to a significant residual uncompensated Doppler shift over this long period of time. In addition, this value of $\epsilon_{\mathrm{AFR}}$ also incorporates all other possible imperfections of the system (App.C.1) and therefore represents an upper limit for the average misalignment of the returning beam.

The corresponding Doppler shifts amount to $\Delta \nu_{D}=0.8(2.2) \mathrm{kHz}$ for the fastest of our velocity classes $(\langle v\rangle \approx 270 \mathrm{~m} / \mathrm{s}), \Delta \nu_{D}=0.20(56) \mathrm{kHz}$ for the slowest $(\langle v\rangle \approx$ $70 \mathrm{~m} / \mathrm{s})$ and $\Delta \nu_{D}=0.6(1.6) \mathrm{kHz}$ for the average of all velocity classes. The latter result represents the uncertainty due to a potential uncompensated first order Doppler shift for the measurement of the $2 \mathrm{~S}-4 \mathrm{P}$ absolute transition frequency in Sec. 7. 


\section{Line distortions due to the coherent interaction with two laser beams}

${ }^{1}$ While the atoms cross the interaction region, they interact coherently with the two laser beams used for the compensation of the first order Doppler effect as discussed in the previous chapters. The absorption and stimulated emission from and into these beams and the associated change in momentum can lead to a multitude of effects, possibly distorting the fluorescence line shape. In some situations, however not in our particular system as detailed below, this can be described as the well-known dipole and recoil force acting on localized atoms [62]. In this approximation, atoms are attracted by the nodes or antinodes for a red or blue detuned laser, leading to asymmetric light-force induced line distortions and systematic frequency shifts. This mechanism has been described before and was identified as the leading systematic effect of optical spectroscopy of the helium $2^{3} \mathrm{~S}_{1} \rightarrow 2^{3} \mathrm{P}_{0,1,2}$ transitions [55]. Such effects, if present, could potentially mask the verification of the Doppler cancellation shown in Fig. 4.1 and Fig. 4.2.

Treating the external motion classically and the internal degrees of freedom quantum mechanically works only under several conditions [62]. One of them is that the atoms stay well localized on a length scale much smaller than the periodicity of the standing wave, i.e. $\lambda / 2$. This condition is indeed fulfilled for the helium $2^{3} \mathrm{~S}_{1} \rightarrow 2{ }^{3} \mathrm{P}_{0,1,2}$ transition. However, for our $2 \mathrm{~S}-4 \mathrm{P}$ setup this is not the case because of the lower mass, the larger photon momentum, the smaller scale size of the standing wave and the longer interaction time in the laser field. The recoil of a single photon due to the internal superposition of the $2 \mathrm{~S}$ and the $4 \mathrm{P}$ state would generate two distinct classical trajectories that separate by several $\lambda / 2$ upon propagation trough the laser beam.

Simulated transverse velocity distributions for selected delay time windows $\tau$ are shown in Fig. 5.1. The atomic beam is well collimated by diaphragms of $1.9 \mathrm{~mm}$ diameter and $2.1 \mathrm{~mm}$ edge length placed along the beam (see also technical drawings in App. E). The full width at half maximum of the resulting distribution of transverse velocities of the $1 \mathrm{~S}$ atoms is about $5.9 \mathrm{~m} / \mathrm{s}$ in the delay window [10 $\mu \mathrm{s} ; 60 \mu \mathrm{s}$ ], i.e. the first experimental data subset that features large velocity spread.

The fact that excitation rates for the $1 \mathrm{~S}-2 \mathrm{~S}$ transition are larger for atoms that travel under smaller angles with respect to the $243 \mathrm{~nm}$ enhancement cavity axis leads to a further collimation of the $2 \mathrm{~S}$ atoms beam. This can be seen by comparing the velocity spread of the $2 \mathrm{~S}$ atoms and the $1 \mathrm{~S}$ atoms in the same delay time window. For

\footnotetext{
${ }^{1}$ The content of this chapter has been published in [25] and was only slightly modified to fit the general structure of this thesis.
} 


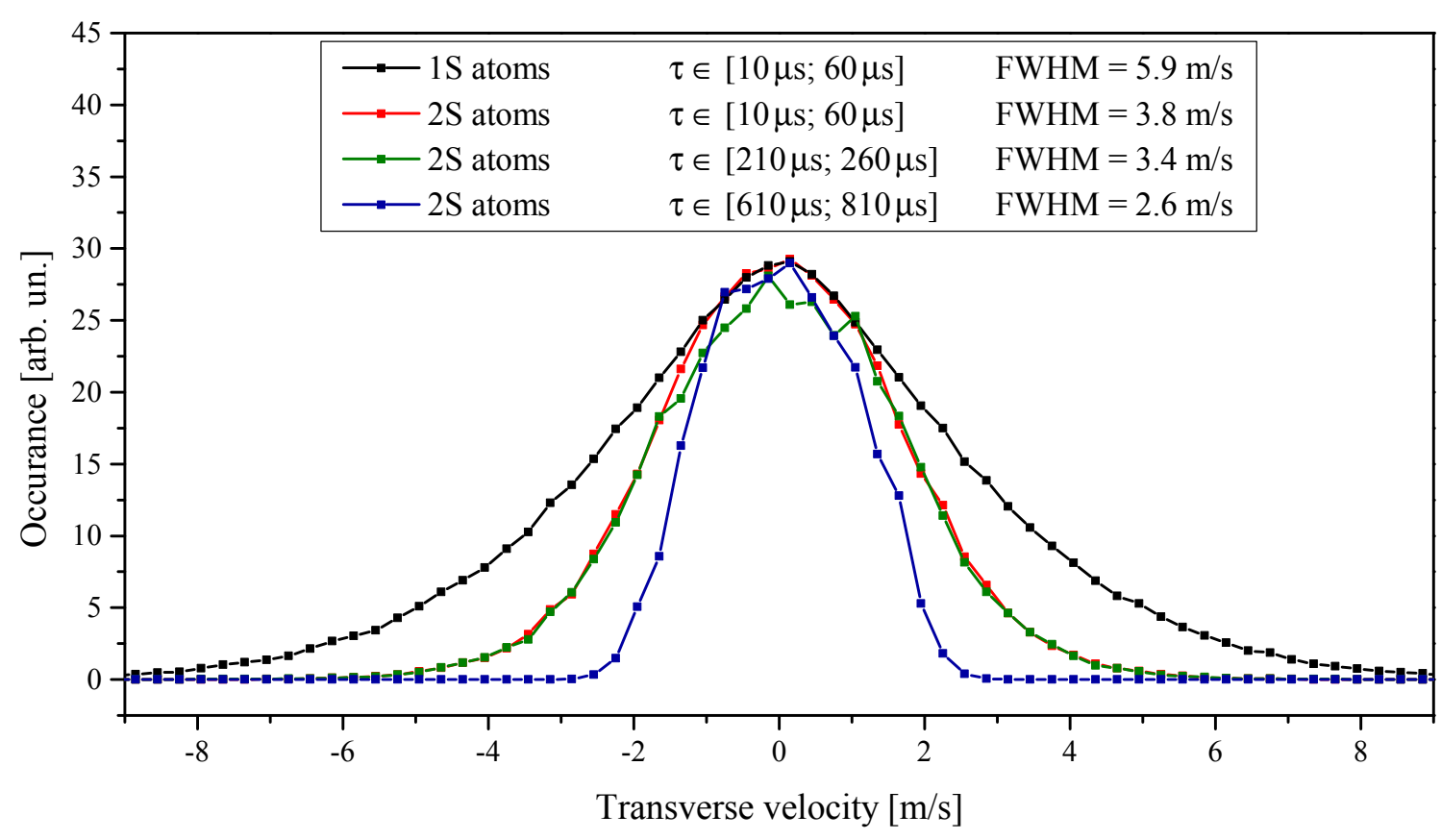

Figure 5.1.: Simulation of transverse velocity distributions ( $10^{5}$ trajectories). Atomic trajectories and $1 \mathrm{~S}-2 \mathrm{~S}$ excitation rates are calculated using the Monte Carlo simulation discussed in Sec.6.2. For the visual comparison of their widths, the different distributions have been normalized to the same amplitude.

the time window $[10 \mu \mathrm{s} ; 60 \mu \mathrm{s}]$ a value of $3.8 \mathrm{~m} / \mathrm{s}(\mathrm{FWHM})$ is found for the $2 \mathrm{~S}$ atoms, whereas the spread of the $1 \mathrm{~S}$ atoms amounts to $5.9 \mathrm{~m} / \mathrm{s}$ (FWHM). The $243 \mathrm{~nm}$ "soft aperture" leads to a decrease of roughly $30 \%$ in this case. At larger delay times, the transverse velocity spread is even smaller due to the smaller longitudinal velocities (Fig. 5.1).

To model the interaction with the two laser beams in this regime the external degrees of freedom of the atoms have to be described by wave mechanics. In this picture coherent superpositions of the momentum eigenstates are generated by the back decay to the $2 \mathrm{~S}$ state. The corresponding two photon recoil gives rise to a small spatial modulation of the atomic density with a period of $\lambda / 2$, that either lines up with the nodes or antinodes of the laser field. Again, this process depends on the laser detuning, giving rise to line shape distortions. In our system, the $4 \mathrm{P}$ atoms predominantly decay to the $1 \mathrm{~S}$ ground state due to the favorable branching ratio and only $4 \%$ of the atoms return to the initial hyperfine $2 \mathrm{~S}$ sub-level $\left(2 \mathrm{~S}_{1 / 2}^{F=0}\right)$. This largely suppresses the spatial modulation of the atomic density.

We have set up a model that describes our system in the quantum regime and found that the corresponding line shifts are negligible for the extraction of the upper limit for the residual uncompensated Doppler shift in Sec.4. In the determination of the $2 \mathrm{~S}-4 \mathrm{P}$ transition frequency in Sec. 7 , the conservative estimate of $1.0 \mathrm{kHz}$ is included in the table of uncertainties (Tab. 7.1). The details on this issue are subject 
to an upcoming publication. Potential asymmetries of our experimental line shape which might be caused by the effects discussed in this section are examined using the expanded line shape (Sec. 2.2) in Sec. 7 and are found to be sufficiently small. 



\section{Measurement of quantum interference line shifts}

\subsection{Experimental data}

During October and November 2014, 7 measurement days for the $2 \mathrm{~S}_{1 / 2}^{F=0} \rightarrow 4 \mathrm{P}_{1 / 2}^{F=1}$ $\left(\nu_{1 / 2}\right)$ transition and 8 days for the $2 \mathrm{~S}_{1 / 2}^{F=0} \rightarrow 4 \mathrm{P}_{3 / 2}^{F=1}\left(\nu_{3 / 2}\right)$ transition have been performed at different orientations $\theta_{L}$ of the linear $2 \mathrm{~S}-4 \mathrm{P}$ excitation light polarization (see Sec.3.3, Fig 2.3 and Fig 3.1). The entire data sets presented in this section consist of 19,720 individual resonances for the $\nu_{1 / 2}$ transition and 26,320 for $\nu_{3 / 2}$ respectively.

\subsubsection{Data acquisition}

Detection of the 2S-4P fluorescence only takes place after the 1S-2S excitation light has been blocked by the chopper wheel depicted in Fig. 3.2. $2 \mathrm{~S}$ atoms travel from the $1 \mathrm{~S}-2 \mathrm{~S}$ excitation region to the $2 \mathrm{~S}-4 \mathrm{P}$ interaction point. At a excitation light power of about $15 \mu \mathrm{W}(6 \mu \mathrm{W})$, a fraction of about $30 \%$ of the atoms in the $2 \mathrm{~S}_{1 / 2}^{F=0}$ state is on average excited to the $4 \mathrm{P}_{1 / 2}^{F=1}\left(4 \mathrm{P}_{3 / 2}^{F=1}\right)$ state and the fluorescence is detected via CEM 1\&2 (Sec. 3). The single photon signals from the detectors are recorded using the time-of-flight resolved detection scheme detailed in Sec. 3.2.

For each measurement setting, i.e. orientation of the linear laser polarization, the angle $\alpha$ between the laser beams and the atomic beam is adjusted to be close to $\alpha=$ $90^{\circ}$ before the actual data acquisition. This is achieved analogous to the description in Sec. 4. Absolute frequency data for the $2 \mathrm{~S}-4 \mathrm{P}$ transition is acquired with active AFR, i.e. open shutter and active stabilization of the HR mirror, for a specific setting of the laser polarization angle $\theta_{L}$. In each line scan taking about $90 \mathrm{sec}$, the resonance is scanned at 30 randomized frequency points [49]. At each frequency point, the signal is integrated over 170 chopper cycles, then the polarization is rotated by $90^{\circ}$ (Sec 3.3) and the signal is integrated over another 170 cycles. In total, a typical measurement day consists of about 100 line scans per polarization direction $\theta_{L}$ and $\theta_{L}+90^{\circ}$, leading to a total number of about 4000 individual resonances per day $(2$ detectors, 2 polarization directions, 10 delays).

\subsubsection{Raw data analysis}

Following the convention in Sec. 2, Voigt functions are fit to the experimental data in order to demonstrate apparent line shifts due to quantum interference. As an 

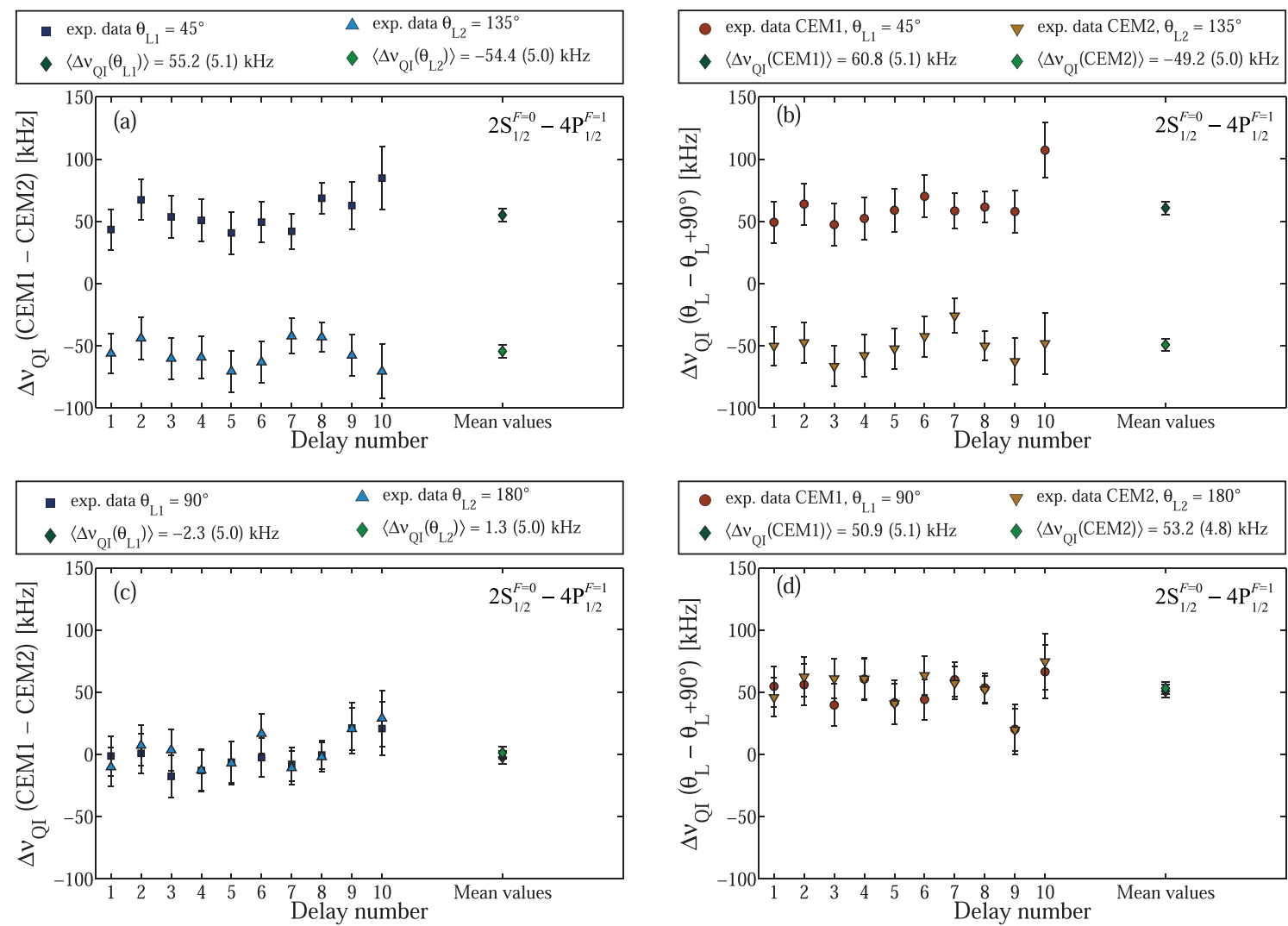

Figure 6.1.: Exemplary data for two measurement days at polarization angles of $\pm 45^{\circ}$ (a, max. difference between detectors) and $90^{\circ} / 180^{\circ}$ (c, zero difference between detectors). b) and d) display the line center difference for each individual split detector at two polarization angles $\theta_{L}=90^{\circ}$ and $\theta_{L}+90^{\circ}$ and serve for consistency checks, e.g. the similarity in the behavior of the two individual detectors. Due to symmetry, the magnitude of the differences at $\pm 45^{\circ}$ in a) and d) as well as the difference at $90^{\circ}$ in d) are expected to be the same. In either case, corresponding results agree.

example, two data sets are shown in Fig. 6.1, corresponding to measurements at $\theta_{L}= \pm 45^{\circ}$, where the difference between the two detectors is maximal (Fig. 6.1a) and $\theta_{L}=90^{\circ}$ and $180^{\circ}$, respectively, where the difference is zero (Fig. 6.1c). For the data shown the following differences in the extracted line centers have been calculated:

Line center difference between detectors CEM 1\&2 $\left(\Delta \nu_{\mathrm{QI}}(\mathrm{CEM} 1\right.$ - CEM2)): The signal possesses a high common noise suppression and high sensitivity to the QI effect due to the complementary signals seen by the two detectors (Sec. 3.2). Possible imperfections of the apparatus that may drift and vary with time, such as a residual Doppler effect or stray electric fields, are common mode and cancel out in the difference. The same is true for faster fluctuations, e.g. the intensities of the $1 \mathrm{~S}-2 \mathrm{~S}$ and $2 \mathrm{~S}-4 \mathrm{P}$ lasers and the flux of hydrogen atoms. 


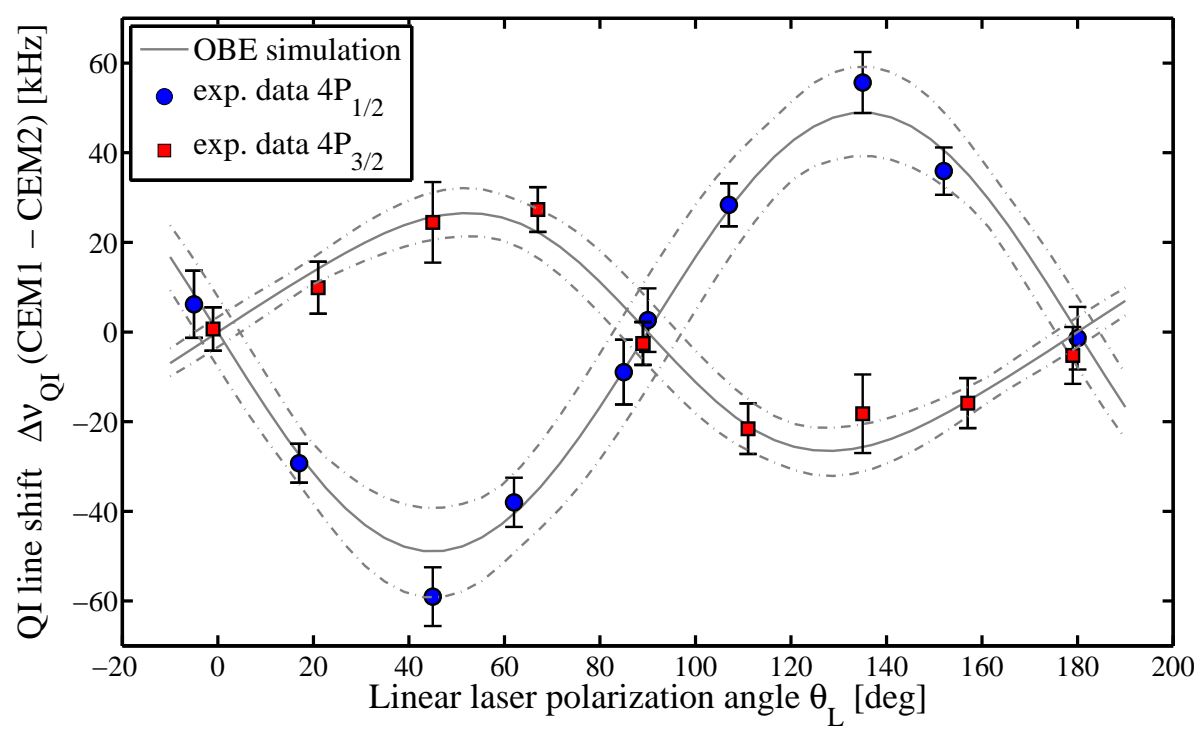

Figure 6.2.: Measurement of apparent line shifts due to quantum interference. Shown are the differences in the Voigt-fit line positions extracted using data obtained from the two detectors CEM1 and CEM2 in Fig. 3.2 as a function of laser polarization for the $2 \mathrm{~S}_{1 / 2}^{F=0} \rightarrow 4 \mathrm{P}_{1 / 2}^{F=1}$ transition (blue circles) and the $2 \mathrm{~S}_{1 / 2}^{F=0} \rightarrow 4 \mathrm{P}_{3 / 2}^{F=1}$ transition (red squares). Differences of up to $\pm 51 \mathrm{kHz}$ in the extracted line centers are observed. These apparent shifts are large on the scale of the proton size puzzle of about $8.9 \mathrm{kHz}$ for the $2 \mathrm{~S}-4 \mathrm{P}$ transition. Simulation results (OBE, Sec 6.2) are shown in gray. Dashed lines indicate confidence bounds for the simulation.

Line center difference at angles $\boldsymbol{\theta}_{\mathbf{L}}$ and $\left(\boldsymbol{\theta}_{\mathbf{L}}+\mathbf{9 0}^{\circ}\right)\left(\Delta \nu_{\mathrm{QI}}\left(\theta_{L}-\theta_{L}+90^{\circ}\right)\right)$ : Fig. $6.1 \mathrm{~b}$ ) and Fig. $6.1 \mathrm{~d}$ ) compare the line centers of the same detector at the two polarization angles $\theta_{L}$ and $\theta_{L}+90^{\circ}$. This signal provides a strong consistency check and a powerful tool to look for certain imperfections in the system. Due to the symmetry of the problem, the differences in Figs. 6.1 a) \& b) are expected to be the same and the data sets agree.

For either case, no significant dependence of the calculated differences on the delay number, and hence the atom's velocity, can be found and the delay average is used for further analysis.

\subsubsection{Observed QI line shifts}

The difference in extracted line centers from the two detectors CEM $1 \& 2$ is depicted in Fig. 6.2 as a function of linear laser polarization angle $\theta_{L}$ for the $2 \mathrm{~S}_{1 / 2}^{F=0} \rightarrow 4 \mathrm{P}_{1 / 2}^{F=1}$ transition (blue points) and for the $2 \mathrm{~S}_{1 / 2}^{F=0} \rightarrow 4 \mathrm{P}_{3 / 2}^{F=1}$ (red squares). Despite the fact that the interfering resonances are separated by about 100 line widths, line shifts due to quantum interference can unambiguously be identified our data. This measurement represents the first direct observation of these line shifts for resonances with a large separation in precision spectroscopy data. 
The features of QI line shifts as expected from the discussion in Sec. 2.4 are found: The extracted line center frequency changes with laser polarization angle $\theta_{L}$ and has a period of $180^{\circ}$. For $2 \mathrm{~S}^{-} 4 \mathrm{P}_{1 / 2}$, it can be well modeled by a sine function. For the $2 \mathrm{~S}-4 \mathrm{P}_{3 / 2}$ transition this is not the case due to the anisotropic emission. For comparable excitation rates, the effect is smaller for $2 \mathrm{~S}-4 \mathrm{P}_{3 / 2}$ than for $2 \mathrm{~S}-4 \mathrm{P}_{1 / 2}$ and of opposite sign as discussed in Sec. 2.4.3.

The observed differences of up to $\pm 51 \mathrm{kHz}$ are large compared to the proton size puzzle that amounts to about $8.9 \mathrm{kHz}$ for the $2 \mathrm{~S}-4 \mathrm{P}$ transition. The line distrotions due to quantum interference and accompanying shifts were modeled using the masterequation approach introduced in Sec. 2.2.3 (OBE, gray line in Fig. 6.2). Experimental details which need to be included in the OBE simulation are discussed in the next section. For the extraction of the $2 \mathrm{~S}-4 \mathrm{P}$ absolute transition frequency in Sec. 7 , the line shape distortions are accounted for by the proper fit function introduced in Sec. 2.2.1.

The line centers extracted from the Voigt fit in this section are used in App. A.2 to demonstrate the cancellation of QI line shifts in the calculation of the 4P fine structure centroid as suggested in Sec. 2.4.3. The shifts may also be corrected by simulation results (see App.A.3), which has up to now been the common way of dealing with the effect.

\subsection{Simulation of experimental line shapes}

Experimental line shapes have been simulated by A. Matveev using high power computation resources provided by the MPG/IPP Rechenzentrum Garching, similar to the discussions in $[8,9,27-29,41]$. In order to recover experimental line shapes, a number of additional features specific to the experimental geometry have to be implemented in the theory described in Sec. 2.2. The final description of the experimental data using the OBE model with all experimental parameters included is quite involved. The differences between individual layers of complexity are illustrated in Fig. 6.3.

The most simplified case has been modeled using the perturbative model (light gray dashed line): The QI line shifts are described for atoms at rest, in the limit of small excitation light power and a finite detection opening angle $\theta_{c}=65^{\circ}$, which corresponds to the geometrical opening angle of the detector. The major part of the QI line shifts observed in the experiment is already described by this rough approximation. Additional corrections are added by the effects described below, which have been implemented for the OBE simulation only because the perturbative approach can not describe all of them, especially the effects of optical pumping and saturation. The additional corrections, however, are considerably smaller and hence do not have to be known extremely accurate.

More complexity is added when using the OBE model. Effects of optical pumping and saturation are automatically included. In the low power limit, the results 


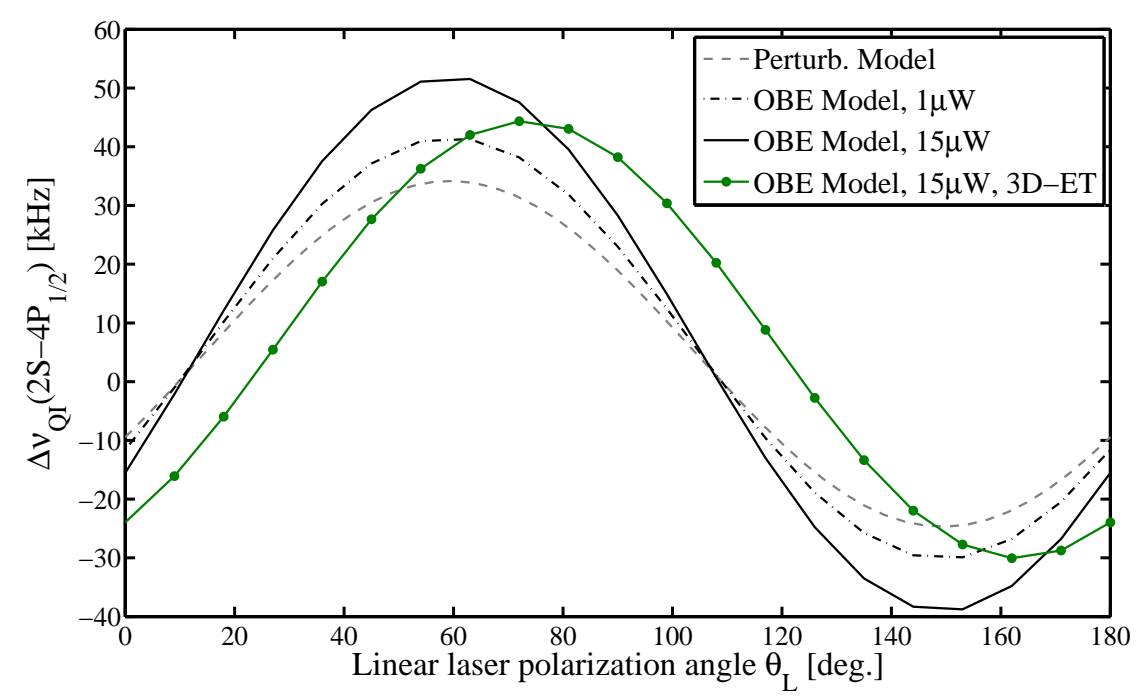

Figure 6.3.: Comparison of calculated QI line shifts for the $2 \mathrm{~S}_{1 / 2}^{F=0} \rightarrow 4 \mathrm{P}_{1 / 2}^{F=1}$ transition and detection opening angle $\theta_{c}=65^{\circ}$. The simplest approximation is given by the perturbative model (grey dashed line) and accounts for about $78 \%$ of the total shift already (compare to green line, full model). Effects due to atomic beam parameters (beam divergence and velocity distribution of atoms) contribute $17 \%$, see black dashed-dotted line. Saturation and power broadening at the experimental power of $15 \mu \mathrm{W}$ increase the shift by $26 \%$ (black solid line). Including the maps of local detection efficiency discussed in App. B.2 (Fig. B.3) into the simulation decreases the predicted shifts by about $21 \%$ (green, 3D-ET). The full model shown in green here is also used in Fig. 6.2. The modification of the effective detection opening angle by inclusion of the emission angle dependent detection efficiency leads to shift of the curve on axis of abscissas and is expected for the used split detectors.

coincide with the ones obtained by the perturbative approach (Sec. 2.3.3).

In addition, the parameters of the atomic beam are modeled. Hydrogen atoms emerge from a cold $(T=5.8 \mathrm{~K})$ copper nozzle in the experiment. Previous studies show that the distribution of velocities is well described by a Maxwell-Boltzmann distribution $[6,47]$. Accordingly, the flux of ground state atoms $\Phi(1 S, v)$ in direction of the beam axis can be written as the product of velocity and the respective probability distribution:

$$
\Phi(1 S, v) \propto v \times v^{2} \exp \left[-\frac{m v^{2}}{2 k T}\right],
$$

where $k$ denotes the Boltzmann constant. The emission angles of the atoms from the nozzle is modeled by a cosine distribution. A Monte Carlo simulation, which has also been used in $[6,7]$, calculates the excitation probability $p(2 \mathrm{~S})$ to the metastabe $2 \mathrm{~S}$ state. Subsequently, the trajectories are fed into a second simulation which calculates the excitation rates of the $2 \mathrm{~S}-4 \mathrm{P}$ transition for the experimental geometry introduced in Sec. 3. A grid $G(\alpha, P, v)$ of $2 \mathrm{~S}-4 \mathrm{P}$ resonances is calculated 
for a set of parameters $\alpha$ (laser-to-atomic-beam angle), $P$ (excitation light power) and $v$ (atom velocity) and each fine structure component. For the computational intensive calculations we utilize facilities provided by the Max Planck Institute of Plasma Physics. Using about 40 cores, the calculation of a grid with 6000 resonances evaluated at 80 frequency points takes about 2 weeks.

Experimental line shapes are reconstructed by interpolation of the grid $G(\alpha, P, v))$ for the trajectories used in the $1 \mathrm{~S}-2 \mathrm{~S}$ simulation and weighting by $p(2 \mathrm{~S})$ accordingly. The emission of Lyman- $\alpha$ and Lyman- $\gamma$ photons is calculated for the three dipole radiation modes. Calculating QI line shifts for a low excitation light power of $1 \mu \mathrm{W}$ and including the atomic beam parameters results in the simulation data depicted by the black dash-dotted line in Fig. 6.3. The expected QI line shifts increase in amplitude by $6.2 \mathrm{kHz}$, i.e. about $17 \%$ of the amplitude of the full model (green), due to additional Doppler broadening of the resonance brought by the atomic beam divergence and the atoms' velocity distribution. The same calculation at the experimental power of $15 \mathrm{\mu W}$ results in a further increase of the QI line shift amplitude by $9.5 \mathrm{kHz}$, i.e. about $26 \%$, due to power broadening and saturation. The effect of power broadening and saturation on the QI residuals is illustrated in Fig. 6.4.

To fully recover the QI line shifts observed in the experiment, the OBE simulation has also to include the efficiency of the XUV detectors discussed in Sec.3.2. The maps of local detection efficiency as shown in Fig. B.3 are used in the full description used in Sec. 6.1.3. The difference to the previous step is $-7.9 \mathrm{kHz}$, i.e. $21 \%$ of the amplitude in the full treatment.

The observed QI line shifts may be affected by additional effects, such as potential reflection of XUV photons and photo electrons from the graphite coated inner walls or a polarization dependence of the photo electron emission efficiency. Many of these additional effects evade a stringent theoretical description but may at least in part be limited by experimental observations (see App. B). Uncertainties are therefore difficult to quantify precisely and an uncertainty of $15 \%$ is assigned to the predicted OBE shifts in Sec. B. The uncertainty, however, is still small enough, so that correcting the line centers obtained by using usual Voigt fits in the previous section can be used for a consistency check of the absolute frequency determination in Sec. 7 in App. A.3.

It is worth to note again, that the extraction of the unperturbed $2 \mathrm{~S}-4 \mathrm{P}$ transition frequency in Sec. 7 as well as the derived values for the Rydberg constant and proton size in Sec. 8 do not rely on the accurate modeling of the QI line shifts. The fit of Eq. (2.8) used for the determination of the line centers accounts for the effects of QI, independent of the exact experimental geometry (see Sec. 2.2.1 and Sec. 7.4.3).

For the OBE simulation, on the other hand, it is important to note, that excellent agreement between the simulation and the experimental data is achieved implementing only a small number of basic parameters, without additional tuning of the parameters. 

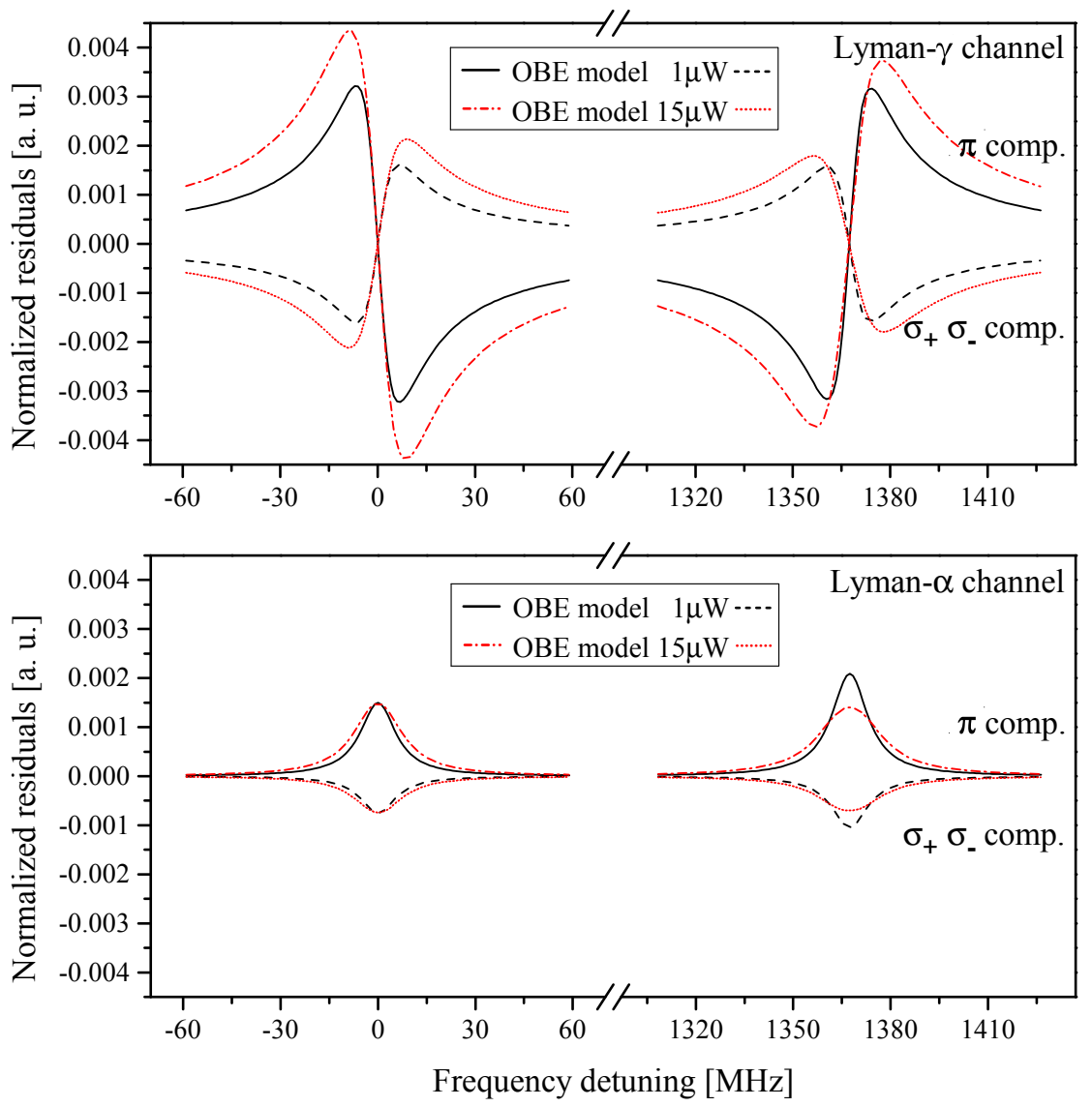

Figure 6.4.: Normalized residuals calculated from the OBE simulation at two different power levels (similar to Fig. 2.5 and Fig. 2.7). Residuals in the Lyman- $\gamma$ channel increase both, in width (power broadening) and in amplitude (saturation) when the excitation light power is increased from $1 \mu \mathrm{W}$ (coincides with perturbation model, see Fig. 2.5) to $15 \mu \mathrm{W}$, the excitation light power used in the experiment. Note that outside the low power limit the QI residuals are no longer equal in amplitude for both transitions due to saturation effects. The additional increase is twice as large for the $2 \mathrm{~S}-4 \mathrm{P}_{1 / 2}$ transition than for the $2 \mathrm{~S}-4 \mathrm{P}_{3 / 2}$ transition. The increase in the amplitudes of the quantum interference residuals relative to the line amplitude directly translates to an increase in the QI line shifts of the apparent line center position as can be seen from Fig. 6.3 (compare OBE Model simulation at $1 \mu \mathrm{W}$ and $15 \mu \mathrm{W}$ of excitation light power). For different power levels (up to $15 \mu \mathrm{W}$ ) the residuals in the Lyman- $\alpha$ channel are always found to be symmetric about the line center. 



\section{Measurement of the 2S-4P absolute transition frequency}

\subsection{Data evaluation}

While the usual fit of a Voigt function discussed in Sec. 6 ( $\xi=0$ in Eq. (2.8)) suggests accurate determination of the line centers by small fit uncertainties, large geometrydependent systematic shifts of the extracted line centers are observed (Fig. 7.4). The full line shape model in Eq. (2.8), where $\xi$ is a free fit parameter on the order of 1 part in $10^{3}$ of the line amplitudes in our data, reduces the residual uncertainty to $0.5 \mathrm{kHz}$. This has been validated by our simulation data (App. 7.4.3). Simulations covering a wide parameter space around the experimental values have been performed and the robustness of the line center extraction by a fit of Eq. (2.8) has been tested. The line shape model is used in this section to determine the $2 \mathrm{~S}-4 \mathrm{P}$ absolute transition frequency.

\section{2. $2 \mathrm{~S}-4 \mathrm{P}$ absolute transition frequency}

The absolute transition frequencies from the $2 \mathrm{~S}_{1 / 2}^{F=0}$ state to the $4 \mathrm{P}_{1 / 2}^{F=1}$ state $\left(\nu_{1 / 2}\right)$ and the $4 \mathrm{P}_{3 / 2}^{F=1}$ state $\left(\nu_{3 / 2}\right)$ depicted in Figure 2.4 have been measured. The data presented in this chapter is identical to the one that has been used for the demonstration of QI line shifts in Sec. 6.

Line centers are determined by a fit of Eq. (2.8) to the experimental data and the mean values of the extracted centers for individual measurement blocks and both hyperfine transitions are depicted in Fig. 7.1. A blind analysis of the data has been performed and the absolute values of the transition frequencies were only revealed after fixing the uncertainties of the leading order systematic effects. The resonance positions, corrected for the recoil shift of $\Delta \nu_{\text {recoil }}=837.23 \mathrm{kHz}$ (Sec. 7.4.8), extracted from the average of both detectors CEM1 and CEM2 in are:

$$
\begin{aligned}
& \nu_{1 / 2}=616520152556.1(1.4)^{\text {stat }}(1.9)^{\mathrm{sys}} \mathrm{kHz} \\
& \nu_{3 / 2}=616521519988.8(1.4)^{\text {stat }}(1.9)^{\mathrm{sys}} \mathrm{kHz}
\end{aligned}
$$

To make full use of the fact that the quantum interference, as well as the effects of saturation and optical pumping, apparently shift the two resonances in opposite directions, it is advantageous to determine the transition frequency from the $2 \mathrm{~S}$ 


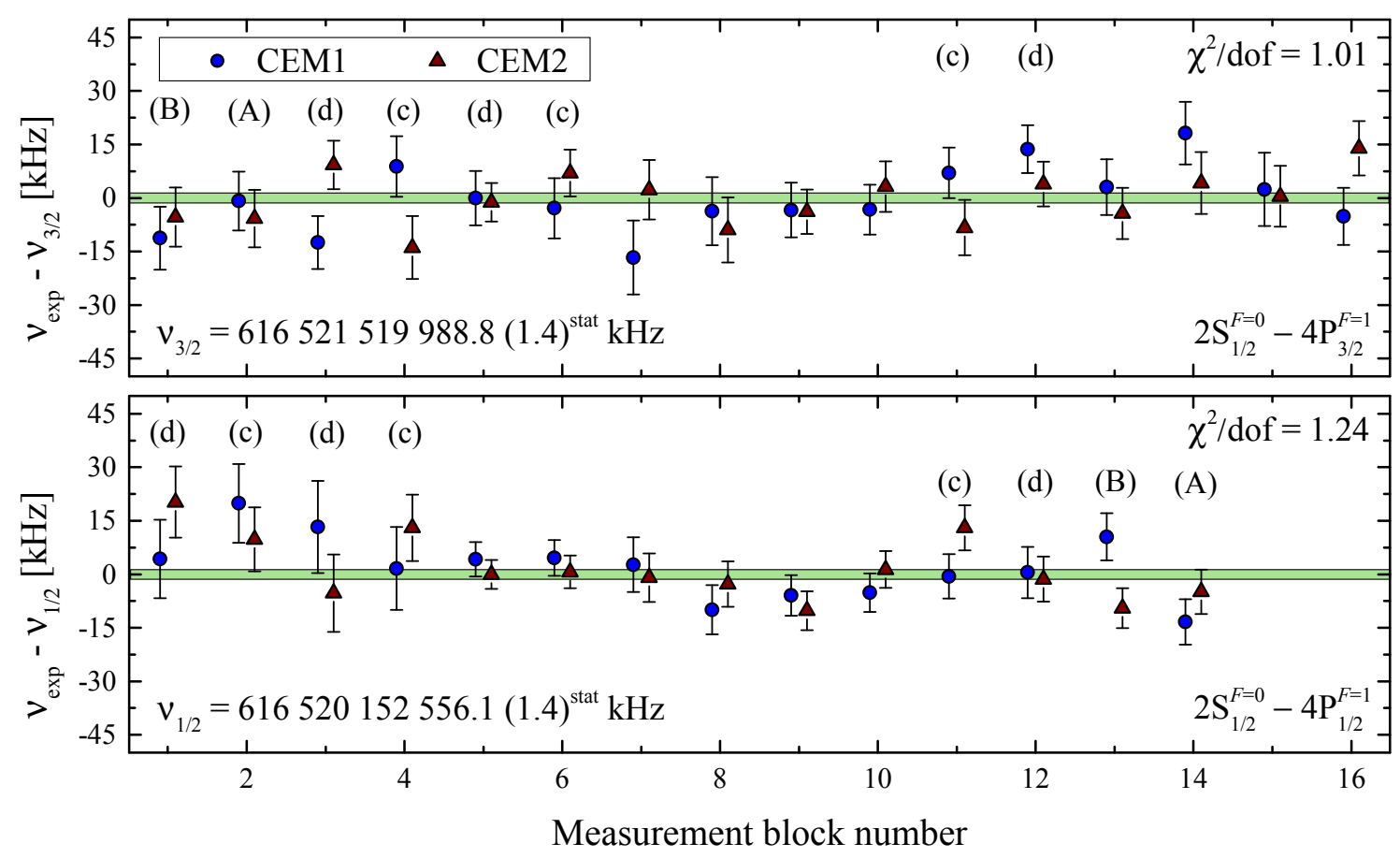

Figure 7.1.: Experimental line centers determined by a fits of Eq. (2.8). Each measurement block consists of an average number of 80 resonances recorded simultaneously for 10 individual velocity classes (Sec.3.2). Error bars indicate the statistical uncertainty (standard error) for each measurement block $\nu_{\exp }(j)$ and the green bands for the extracted absolute frequencies of the $\nu_{1 / 2}\left(2 \mathrm{~S}_{1 / 2}^{F=0}-4 \mathrm{P}_{1 / 2}^{F=1}\right.$, bottom $)$ and $\nu_{3 / 2}$ transition $\left(2 \mathrm{~S}_{1 / 2}^{F=0}-4 \mathrm{P}_{3 / 2}^{F=1}\right.$, top $)$, respectively.

hyperfine centroid to the $4 \mathrm{P}$ fine structure centroid (i.e. the centroid of the hyperfine centroids, see Sec. 7.4.10) using Eq. (7.1) and Eq. (7.2):

$$
\nu_{2 S-4 P}=616520931625.8(1.0)^{\mathrm{stat}}(1.9)^{\mathrm{sys}} \mathrm{kHz}
$$

where "stat" indicates the statistical and "sys" indicates the systematic uncertainties. The uncertainties are added in quadrature to yield the final uncertainty of $2.2 \mathrm{kHz}$, improving the previous best measurements of the $2 \mathrm{~S}_{-}-4 \mathrm{P}_{1 / 2}$ and $2 \mathrm{~S}-4 \mathrm{P}_{3 / 2}$ transitions [5] by factors of 6.8 and 4.5 , respectively.

The uncertainty of the first order Doppler effect deduced in [25] is the same and correlated for $\nu_{1 / 2}$ and $\nu_{3 / 2}$. The Doppler uncertainty dominates the overall systematic uncertainty, so that Eq. (7.1), Eq. (7.2) and Eq. (7.3) feature identical systematic uncertainties. The systematic effects are discussed in Sec. 7.4 and Eq. (7.3) is used in Sec. 8 to determine the Rydberg constant and proton charge radius. 


\subsection{P fine structure interval}

The measured absolute transition frequencies $\nu_{1 / 2}$ (Eq. (7.1)) and $\nu_{3 / 2}$ (Eq. (7.2)) are used to determine the fine structure splitting $\Delta \nu_{F S}^{\exp }(4 \mathrm{P})$ between the $4 \mathrm{P}_{1 / 2}^{F=1}$ and $4 \mathrm{P}_{3 / 2}^{F=1}$ state:

$$
\Delta \nu_{F S}^{\exp }(4 \mathrm{P})=1367432.7(2.0)^{\mathrm{stat}}(1.9)^{\mathrm{sys}} \mathrm{kHz} .
$$

This can be compared to the theoretical value of $\Delta \nu_{F S}^{\text {theo }}(4 \mathrm{P})=1367433.3(3) \mathrm{kHz}$ (for details on $\Delta \nu_{F S}^{\text {theo }}(4 \mathrm{P})$, see Sec. 7.4.10). Fine structure intervals are almost entirely free from finite size corrections and can be calculated very precisely [4, 43-45]. With a difference of $-0.6(2.8) \mathrm{kHz}$, our experimental result is in excellent agreement with the theoretical value and represents the most accurate optical determination of a fine structure splitting in atomic hydrogen.

Moreover, since the shifts due to quantum interference for $\nu_{1 / 2}$ and $\nu_{3 / 2}$ feature opposite sign (see discussion of Eq. (2.6)), this value represents a sensitive probe for residual quantum interference shifts and an independent test of the internal consistency of our data analysis.

\subsection{Systematic effects}

${ }^{1} \mathrm{~A}$ full list of studied systematic effects for $2 \mathrm{~S}-4 \mathrm{P}$ spectroscopy is given in Tab. 7.1, while the following sections summarize the methods applied to limit the individual effects.

\subsubsection{First order Doppler shift}

The residual uncompensated Doppler shift using the active fiber-based retroreflector (Sec. 3.5) in combination with the cryogenic source of hydrogen $2 \mathrm{~S}$ atoms (Sec. 3.1) has been characterized in a separate study (see Sec.4). Averaging of the ten data subsets with different mean velocities of the contributing atoms, ranging between about $270 \mathrm{~m} / \mathrm{s}$ and $70 \mathrm{~m} / \mathrm{s}$, results in an uncertainty of $1.6 \mathrm{kHz}$ for the residual uncompensated shift.

\subsubsection{Radiation force induced line distortions}

As discussed in Sec. 5, potential asymmetries due to the coherent interaction of our sample atoms with the two laser beams used for the cancellation of the first order Doppler effect are expected to be sufficiently small according to preliminary simulations of this effect. This section examines asymmetric line distortions other than the ones caused by quantum interference in the experimental data using Eq. (2.8).

\footnotetext{
${ }^{1}$ Fractions of this section have been included in the supplementary information to [A. Beyer et al., "The Rydberg constant proton size from atomic hydrogen", in preparation (2016)].
} 


\begin{tabular}{|c|c|c|c|}
\hline & $\Delta v[\mathrm{kHz}]$ & $\sigma[\mathrm{kHz}]$ & $\sigma / v_{2 S-4 P}\left[10^{-12}\right]$ \\
\hline Statistics & 0.0 & 1.0 & 1.6 \\
\hline First order Doppler shift & 0.0 & 1.6 & 2.6 \\
\hline Radiation force induced line distortions & 0.0 & 1.0 & 1.6 \\
\hline Quantum interference & 0.0 & 0.5 & 0.8 \\
\hline Second order Doppler shift & 0.0 & 0.25 & 0.4 \\
\hline DC Stark effect & 0.0 & 0.2 & 0.3 \\
\hline Optical pumping and saturation & 0.0 & 0.1 & 0.2 \\
\hline AC Stark effect & 0.0 & 0.1 & 0.2 \\
\hline Laser spectrum & 0.0 & 0.1 & 0.2 \\
\hline Laser frequency determination & 0.0 & 0.1 & 0.2 \\
\hline Frequency standard: $\mathrm{H}$ maser & 0.0 & 0.06 & 0.1 \\
\hline Hyperfine structure (HFS) corrections & -132552.092 & 0.075 & 0.12 \\
\hline Incoherent line pulling $(P=0 \mu \mathrm{W})$ & 0.0 & 0.08 & 0.1 \\
\hline Recoil shift & -837.23 & 0.000 & 0.0 \\
\hline Zeeman effect & 0.0 & 0.004 & 0.006 \\
\hline Off-diagonal HFS corrections & 0.0 & 0.003 & 0.005 \\
\hline Total & -133389.32 & 2.2 & 3.6 \\
\hline
\end{tabular}

Table 7.1.: Budget of corrections $\Delta \nu$ and uncertainties $\sigma$ for the determination of the $2 \mathrm{~S}-4 \mathrm{P}$ absolute transition frequency $\nu_{2 S-4 P}$ in Eq. (7.3).

First, the expanded line shape in Sec. 2.3.2 is used to illustrate the applied procedure, which is subsequently generalized to apply to the experimental case.

The parameters $a_{4}$ in Eq. (2.24) measure the amplitude of the dispersive shaped line distortions due to quantum interference. They depend on the linear laser polarization angle $\theta_{L}$ and feature opposite sign for the $2 \mathrm{~S}-4 \mathrm{P}_{1 / 2}$ and $2 \mathrm{~S}-4 \mathrm{P}_{3 / 2}$ transitions:

$$
a_{4}^{\left(4 P_{3 / 2}\right)}\left(\theta_{L}\right)=-a_{4}^{\left(4 P_{1 / 2}\right)}\left(\theta_{L}\right) .
$$

The radiation force induced line shape distortions, on the other hand, do not feature this $\theta_{L}$ dependence, because of the proportionality of the force either to the local gradient of intensity or the intensity of the excitation light. If present, these additional (dispersive shaped [63]) distortions would manifest in a constant offset $\zeta$ of the $a_{4}$ parameters, such that averaging over $\theta_{L}$ yields:

$$
\left\langle a_{4}^{\left(4 P_{1 / 2}\right)}\left(\theta_{L}\right)+\zeta+a_{4}^{\left(4 P_{3 / 2}\right)}\left(\theta_{L}\right)+\zeta\right\rangle_{\theta_{L}}=\zeta
$$

Due to the finite detection opening angle, atomic beam divergence and excitation light power in the experiment, the experimentally observed parameters $\tilde{a}_{j}$ do not follow the same simple analytic expressions given in Sec. 2.3.2. These only apply to the case of fluorescence from a single atomic dipole detected by an infinitesimally small detector in the low power limit (see Sec.6.2 and Fig. 6.4). However, the same arguments apply. The line shape model in Eq. (2.8) measures the parameters $\tilde{a}_{4}$ and 

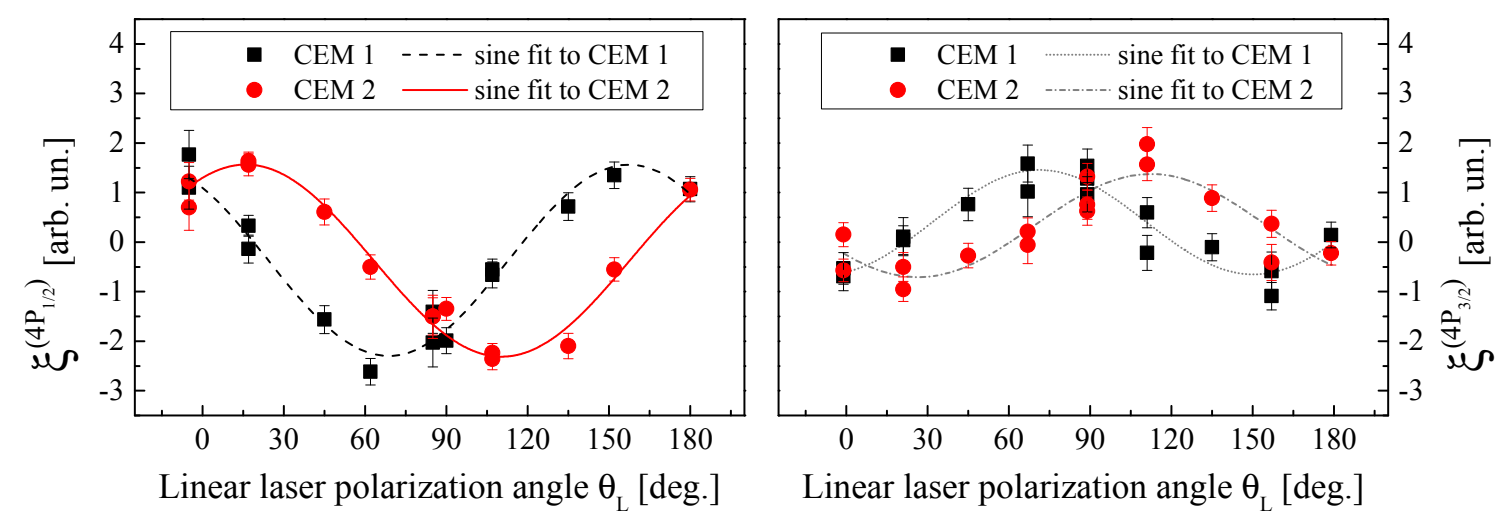

Figure 7.2.: Parameters $\xi$ extracted from experimental data using the expanded line shape model function in Eq. (2.8). Due to the isotropic emission from the $4 \mathrm{P}_{1 / 2}^{F=1}$ state, $\xi^{\left(4 P_{1 / 2}\right)}$ is described by a sine function (black dashed and red lines on left side). This is not the case for $\xi^{\left(4 P_{3 / 2}\right)}$ due to the anisotropic emission from the $4 \mathrm{P}_{3 / 2}^{F=1}$ state. The gray lines are therefore only auxiliary approximations to visualize the opposite sign and different amplitudes of $\xi^{\left(4 P_{1 / 2}\right)}$ and $\xi^{\left(4 P_{3 / 2}\right)}$.

$\zeta$ in units of the respective line amplitudes $\tilde{a}_{1}$, denoted by the parameter $\xi$ :

$$
\xi\left(\theta_{L}\right)=\frac{\tilde{a}_{4}\left(\theta_{L}\right)+\zeta}{\tilde{a}_{1}\left(\theta_{L}\right)},
$$

and an analogous expression to Eq. (7.6) can be derived to determine $\zeta$ :

$$
\left\langle\frac{\tilde{a}_{1}^{\left(4 P_{1 / 2}\right)}\left(\theta_{L}, P_{1}\right) \xi^{\left(4 P_{1 / 2}\right)}\left(\theta_{L}, P_{1}\right)+\tilde{a}_{1}^{\left(4 P_{3 / 2}\right)}\left(\theta_{L}, P_{2}\right) \xi^{\left(4 P_{3 / 2}\right)}\left(\theta_{L}, P_{2}\right)}{\tilde{a}_{1}^{\left(4 P_{1 / 2}\right)}\left(\theta_{L}, P_{2}\right)+\tilde{a}_{1}^{\left(4 P_{3 / 2}\right)}\left(\theta_{L}, P_{2}\right)}\right\rangle_{\theta_{L}}=\zeta
$$

where $P_{1}$ and $P_{2}=P_{1} / 2$ denote the excitation light powers used for the spectroscopy of the $2 \mathrm{~S}-4 \mathrm{P}_{1 / 2}$ and $2 \mathrm{~S}-4 \mathrm{P}_{3 / 2}$ transitions, respectively. In essence, this approach represents a re-formulation of the center of weight method discussed for the cancellation of QI line shifts in Sec. 2.4.3.

The procedure has been tested using pseudo data simulated in the master-equation approach (Sec. 6.2). Residual errors in recovering the known input parameters utilizing Eq. (7.8) for the fit parameters obtained using the line shape model in Eq. (2.8) have been found to be largely independent of the specific simulation used. These include simulations at power levels between $1 \mu \mathrm{W}$ and $15 \mu \mathrm{W}$ and for the ten delay time windows (i.e. velocity distributions) used in the experiment. The residual frequency errors are less than $0.02 \mathrm{kHz}$ in all cases studied. If the experimental power levels $P_{1}=15 \mu \mathrm{W}$ and $P_{2}=6 \mu \mathrm{W}$ are used (which do not obey the assumption $P_{2}=P_{1} / 2$ above), the residual errors are slightly larger, but well within $0.1 \mathrm{kHz}$.

Parameters $\xi$ extracted from the experimental data at different laser polarization angles are shown in Fig. 7.2. The experimental line strengths $\tilde{a}_{4}\left(\theta_{L}\right)$ are described by the OBE simulation in Sec. 6.2 with sufficient accuracy for the purposes of this section 
(deviations $<3 \%$, Fig. B.2). They are used for the weighting of the experimental data in Eq. (7.8). The sine amplitude $\xi_{A}^{\left(4 P_{1 / 2}\right)}$ is determined from fits of the experimental data shown on the left side of Fig. 7.2. It corresponds to a $34 \mathrm{kHz}$ shift of the observed line center due to the quantum interference line distortions (Fig. B.1). The additional line distortion $\zeta$ determined via Eq. (7.8) is compared to $\xi_{A}^{\left(4 P_{1 / 2}\right)}$ to obtain an estimate for the frequency shift caused by $\zeta$ :

$$
\Delta \nu_{\zeta}=\frac{\zeta}{\xi_{A}^{\left(4 P_{1 / 2}\right)}} 34 \mathrm{kHz}=0.3(7) \mathrm{kHz}
$$

The additional shift is small and compatible with zero. It is important to note, that this shift has been accounted for by the line shape model. It is therefore implicitly included in the absolute frequency given in Eq. (7.3). The residual uncompensated shift due to higher order corrections of the dispersive radiation force induced line shape distortions may be expected to be smaller than the observed shift.

Due to the $100 \%$ correlation of the extracted parameters $\xi$ and the line centers determined from a fit of Eq. (2.8), the statistical uncertainty of $0.7 \mathrm{kHz}$ in Eq. (7.9) is implicitly included in the statistical uncertainty given in Eq. (7.3).

The above arguments are partly strengthened by the fact, that no significant velocity dependence of the extracted center frequency is observed (Sec.4). Comparing the first data subset in the delay time window $\tau \in[10 \mu \mathrm{s} ; 60 \mu \mathrm{s}]$ with later delay times, the mean longitudinal velocity is decreased by a significant factor of four and the transverse velocity spread by about two third. The longitudinal velocity determines the interaction time and therefore the longitudinal displacement of the atomic wave packed in the standing wave. If present, the effect might naturally be expected to vary as a function of longitudinal and transverse velocity. However, without knowing the functional behavior of the line shifts, a quantitative estimate can not be given by the velocity analysis.

Until the theoretical study is finalized an additional conservative uncertainty of $1.0 \mathrm{kHz}$ is added to the budget of uncertainties (Tab. 7.1) to account for possible higher order corrections to the dispersive shape of the radiation force induced line shape distortions. Summing up this explicit and the implicit contribution in the statistical uncertainty in quadrature, the total uncertainty due to asymmetric line shape modifications included in Eq. (7.3) is $1.2 \mathrm{kHz}$.

\subsubsection{Quantum interference}

The line shape model in Eq. (2.8) has been tested using simulated line shapes from the full OBE description (Sec.6.2), similar to the discussions in the section above. Residual errors in the determination of the line center position fitting pseudo data with Eq. (2.8) are depicted in Fig. 7.4 for two representative simulations (low power regime and experimental power levels). For a wide parameter space (power levels and delay times, see section above), these residual errors are found to be less then 

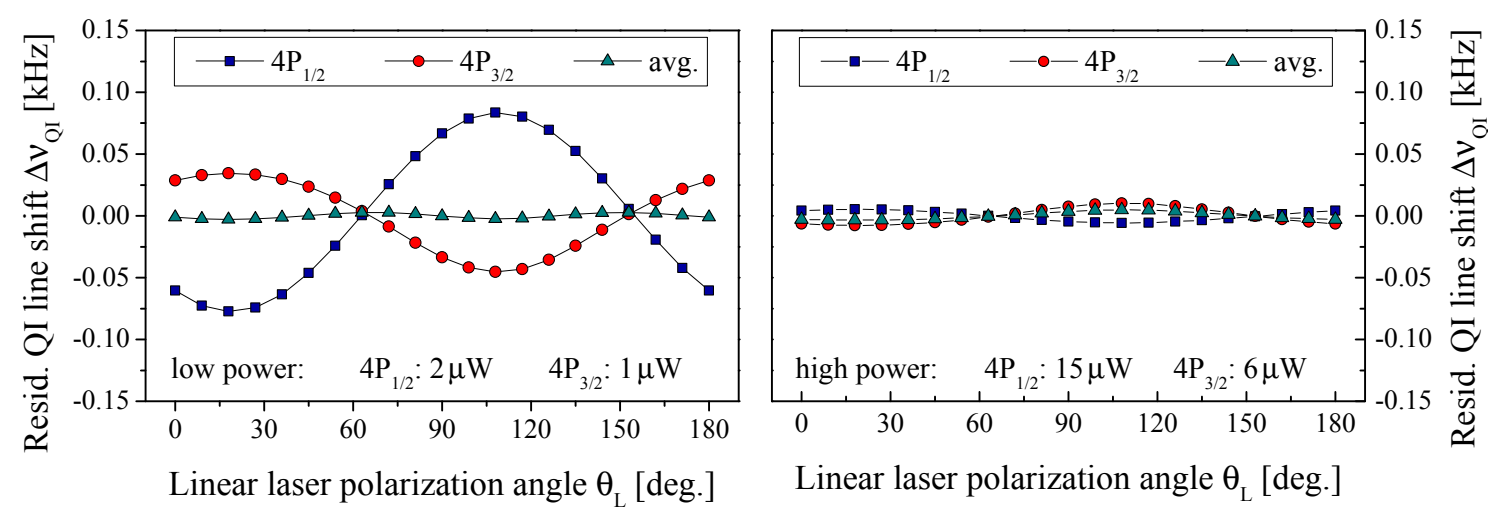

Figure 7.3.: Test of expanded line shape model function Eq. (2.8). Simulated line shapes for the $2 \mathrm{~S}_{1 / 2}^{F=0} \rightarrow 4 \mathrm{P}_{1 / 2}^{F=1}$ transition and $2 \mathrm{~S}_{1 / 2}^{F=0} \rightarrow 4 \mathrm{P}_{3 / 2}^{F=1}$ transition are obtained from the master-equation approach (Sec.6.2) and fit by Eq. (2.8). Left: low excitation light power regime. Right: excitation light powers identical to experiment. Depicted are the residual errors in the extracted line centers. In a wide range of tested simulation parameters, these are less than $0.5 \mathrm{kHz}$. Additional suppression of these small residual errors is observed in the weighted average of both transitions or when averaging over $\theta_{L}$.

$0.5 \mathrm{kHz}$. Moreover, the small residual errors cancel to a large extent when calculating the $4 \mathrm{P}$ fine structure centroid or when averaging over all polarization angles $\theta_{L}$ as performed in the evaluation of the experimental data (green triangles in Fig. 7.4). We have, however, not tested this cancellation in the presence of other imperfections and experimental noise and therefore keep $0.5 \mathrm{kHz}$ as a conservative estimate in the table of uncertainties.

Another way of testing the effectiveness of the line shape model in Eq. (2.8) is to examine the residual difference in the line centers determined from the detectors CEM 1\&2 similar to Fig. 6.2 for the Voigt fits. A comparison of this difference for the Voigt fits and fits of Eq. (2.8) to the $\nu_{1 / 2}$ data is shown in Fig. 7.4. The large $\theta_{L}$ dependence vanishes in the latter case and - in contrast to the Voigt case - the observed scatter of the determined line centers is compatible with one expected from Gaussian distributed data $\left(\chi^{2} /\right.$ dof $\left.=0.82\right)$.

If the data is modeled using (improper) Voigt fit, the large apparent QI line shifts can be still be canceled using the center of weight method discussed in Sec. 2.4.3. This method is applied in App. A.2 and yields compatible results as shown in Fig. 8.1.

\subsubsection{Second order Doppler effect}

The second order Doppler effect is not canceled by the excitation of the $2 \mathrm{~S}-4 \mathrm{P}$ transition utilizing phase-retracing beams. The mean velocities provided by the cryogenic source of $2 \mathrm{~S}$ atoms, however, are small (between about $270 \mathrm{~m} / \mathrm{s}$ to $70 \mathrm{~m} / \mathrm{s}$ ). The average second order Doppler shift amounts to less than $0.25 \mathrm{kHz}$ using the analytic expression: 


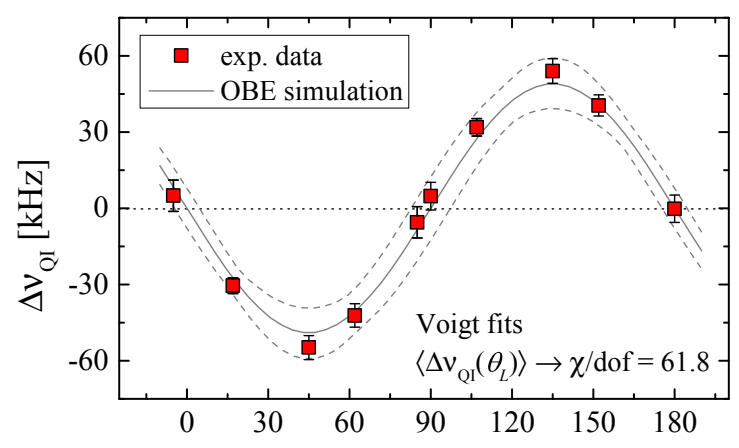

Linear laser polarization anlge $\theta_{L}[\mathrm{deg}$.]

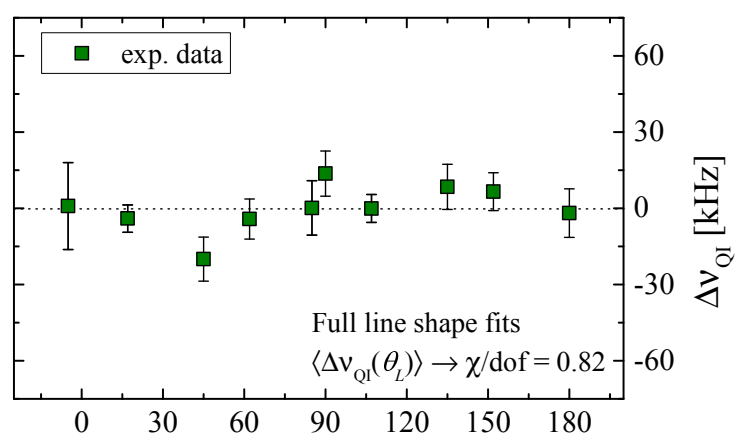

Linear laser polarization anlge $\theta_{L}$ [deg.]

Figure 7.4.: Apparent line shifts due to quantum interference for the $2 \mathrm{~S}_{1 / 2}^{F=0}$ to $4 \mathrm{P}_{1 / 2}^{F=1}$ transition. Depicted is the difference $\Delta \nu_{\mathrm{QI}}$ in the centers determined from the two detectors CEM1 and CEM2 as a function of the linear laser polarization angle $\theta_{L}$ (Fig. 3.1). Improper modeling of the respective data by usual Voigt fits (left) leads to apparent geometry dependent shifts of determined resonances. The behavior is sinusoidal with an amplitude of $51 \mathrm{kHz}$, much larger than the proton size puzzle of $8.9 \mathrm{kHz}$. Experimental findings are well described by our numerical simulation of the excitation dynamics, shown in gray (OBE simulation, Sec. 6.2). Right: Proper modeling of the resonances using Eq. (2.8) absorbs these apparent shifts and provides line centers which are independent of $\theta_{L} \cdot \chi^{2} /$ dof for the average over $\theta_{L},\left\langle\Delta \nu_{Q I}\right\rangle$, is thereby improved from 61.8 (left) to 0.82 (right). dof: degrees of freedom $(\operatorname{dof}=9)$.

$$
\left|\Delta \nu_{\mathrm{SOD}}\right|=\left|-\frac{1}{2}\left(\frac{v}{c}\right)^{2} \nu_{2 S-4 P}\right|<0.25 \mathrm{kHz}
$$

\subsubsection{Stark effect}

Stray electric fields are suppressed by graphite coatings on all surfaces within the spectroscopy regions. An upper limit of $6 \mathrm{mV} / \mathrm{cm}$ has been obtained for the field strength of possible stray electric fields using 1S-2S spectroscopy in the same apparatus $[6,7,47]$. The DC Stark effect of the $4 \mathrm{P}_{3 / 2}^{F=1}$ state is about ten times larger than those for the $4 \mathrm{P}_{1 / 2}^{F=1}$ state (see Tab. 6-3 in [64]). Our re-calculation of the DC Stark coefficients coincides with the numbers given in [64].

An upper limit of $0.5 \mathrm{kHz}$ for the $4 \mathrm{P}_{3 / 2}^{F=1}\left(m_{F}=0\right)$ sub-state is obtained assuming a static electric field of $6 \mathrm{mV} / \mathrm{cm}$ coinciding with the direction of the $486 \mathrm{~nm}$ excitation light electric field vector. This limit is reduced by the fact that the electric field vector is oriented at different angles $\theta_{L} \in\left[0 ; 180^{\circ}\right]$ for different data blocks contributing to the determination of $\nu_{3 / 2}$ in Eq. (7.2). Finally, when calculating $\nu_{2 S-4 P}$, the uncertainty is further reduced by averaging with the much less unaffected $\nu_{1 / 2}$ transition (Eq. (6) in the main text), yielding $0.2 \mathrm{kHz}$ as listed in Tab. 7.1.

A grounded Faraday cage made from a graphite coated stainless steel mesh with two wires (diameter $30 \mu \mathrm{m}$ ) per millimeter surrounds the $2 \mathrm{~S}-4 \mathrm{P}$ interaction volume. 


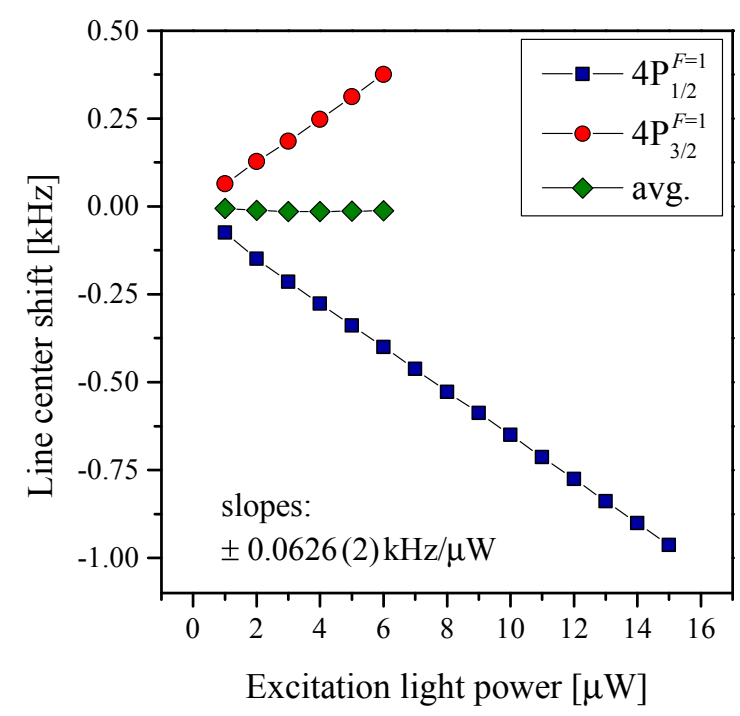

Figure 7.5.: Power dependence of line centers extracted from simulated line shapes. Line shapes are simulated using the master equation approach (Sec.6.2) and fit using Eq. (2.8). A small linear power dependence of $\pm 0.0626(2) \mathrm{kHz} / \mathrm{\mu W}$ is observed and used for the extraction of the $4 \mathrm{P}$ fine structure splitting $\Delta \nu_{F S}^{\exp }(4 \mathrm{P})$ in Eq. (7.4). The shifts for the two $4 \mathrm{P}$ fine structure components $4 \mathrm{P}_{1 / 2}^{F=1}$ and $4 \mathrm{P}_{3 / 2}^{F=1}$ average to zero.

It efficiently shields the electric fields created by the channel electron multiplier input surfaces $(+270 \mathrm{~V}, \mathrm{DC})$ so that the residual fields have negligible influence on the energy levels.

\subsubsection{Laser intensity dependence}

Intensity dependent line shifts have been studied using the OBE Monte Carlo simulation. The observed AC stark effect is less than $0.1 \mathrm{kHz}$. Additional intensity dependences are brought by saturation and optical pumping. The latter is caused by the fact that the excited $4 \mathrm{P}$ states decay back to the $2 \mathrm{~S}$ state with a branching ratio of about $12 \%$ (Balmer- $\beta$, Fig. 2 in main text). One third of these back decays end in the initial $2 S_{1 / 2}^{F=0}$ state, while the remaining two third end in the previously unpopulated $2 S_{1 / 2}^{F=1}$ state. This undesired population re-distribution may, in principle, give rise to excitations of additional $4 \mathrm{P}$ hyperfine components. However, due to the small excitation light power those excitation amplitudes are sufficiently small.

Only for the determination of the $4 \mathrm{P}$ fine structure splitting in Eq. (7.4), small corrections due to optical pumping and saturation are taken from the OBE Monte Carlo simulation (Sec.6.2), estimating an uncertainty of less than 15\%: the measured transition frequencies $\nu_{1 / 2}$ and $\nu_{3 / 2}$ are corrected by $-0.87 \mathrm{kHz}$ and $0.38 \mathrm{kHz}$. Corrections due to the incoherent line pulling are even smaller, yielding $-0.045 \mathrm{kHz}$ and $0.023 \mathrm{kHz}$, respectively.

These corrections are not required for $2 \mathrm{~S}-4 \mathrm{P}$ absolute frequency, since the shifts cancel to a large extent in the determination of the $4 \mathrm{P}$ fine structure centroid. 


\subsubsection{Laser spectrum and frequency}

The spectral purity of the $2 \mathrm{~S}-4 \mathrm{P}$ laser is routinely monitored by a beat note with light from the $1 \mathrm{~S}-2 \mathrm{~S}$ laser at $486 \mathrm{~nm}$. Asymmetries of the laser spectrum that might fold into the observed $2 \mathrm{~S}-4 \mathrm{P}$ line shapes are small due to the use of long external cavity diode lasers (see $[46,48]$ ). We obtain an upper limit of $0.1 \mathrm{kHz}$ by artificially removing the noise pedestal on one side of the measured laser spectrum, numerically folding it into the $2 \mathrm{~S}-4 \mathrm{P}$ line shape and fitting the result.

Laser frequencies are determined via beat notes with an erbium-doped fiber frequency comb which is referenced to an active hydrogen maser. The active hydrogen maser is calibrated via GPS, and its relative frequency uncertainty is better than 1 part in $10^{13}$ at any given time. The maser calibration uncertainty transfers to an uncertainty of less than $0.06 \mathrm{kHz}$ in the $2 \mathrm{~S}-4 \mathrm{P}$ transition frequency and is therefore insignificant at the current level of accuracy.

The absolute laser frequencies are determined by a quadratic fit of the comb beat note data $\nu_{\text {beat }}(t)$ and using Eq. (3.1) and leads to a laser frequency uncertainty of less than $0.1 \mathrm{kHz}$.

\subsubsection{Recoil shift}

Energy and momentum conservation require the absorbed photon energy to be larger than the atomic resonance frequency by the recoil shift of the atom upon absorption. The corresponding recoil shift can be written as:

$$
\Delta \nu_{\text {recoil }}=\frac{h}{2 M_{H}}\left(\frac{\nu}{c}\right)^{2} \approx 837.23 \mathrm{kHz}
$$

and is known with much smaller uncertainty than required here. It can be calculated using the experimental values of the ratio $h / m_{e}$ of the Planck constant $h$ and the electron mass $m_{e}$, the binding energy of the $\mathrm{H}$ atom and the mass of the proton and the electron in atomic mass units [4]. The value of $\nu_{2 S-4 P}$ given in Eq. (7.3) has been corrected for the recoil shift.

\subsubsection{Zeeman Effect}

The earth magnetic field is pre-compensated by three orthogonal pairs of Helmholtz coils outside the vacuum chamber. In addition, the $2 \mathrm{~S}-4 \mathrm{P}$ interaction region is shielded from residual magnetic fields by a double layer high permeability metal ( $\mu$-metal) shield. Thereby, magnetic fields are suppressed to less than $0.35 \mathrm{mG}$ in a volume of about $3 \mathrm{~cm}^{3}$ around the $2 \mathrm{~S}-4 \mathrm{P}$ interaction region.

An upper limit for the residual Zeeman shift of $0.004 \mathrm{kHz}$ is obtained by assuming the transition from the $2 \mathrm{~S}_{1 / 2}^{F=0}$ to the $4 \mathrm{P}_{3 / 2}^{F=1}\left(m_{F}=1\right)$ level was driven by circular polarized light with an amplitude of $1 / 200$ of the desired linear polarization (measured intensity polarization extinction ratio). All other levels involved are at least of factor of ten less sensitive than the $4 \mathrm{P}_{3 / 2}^{F=1}\left(m_{F}=1\right)$ state (shift $2.3 \mathrm{MHz} / \mathrm{G}$ ). 


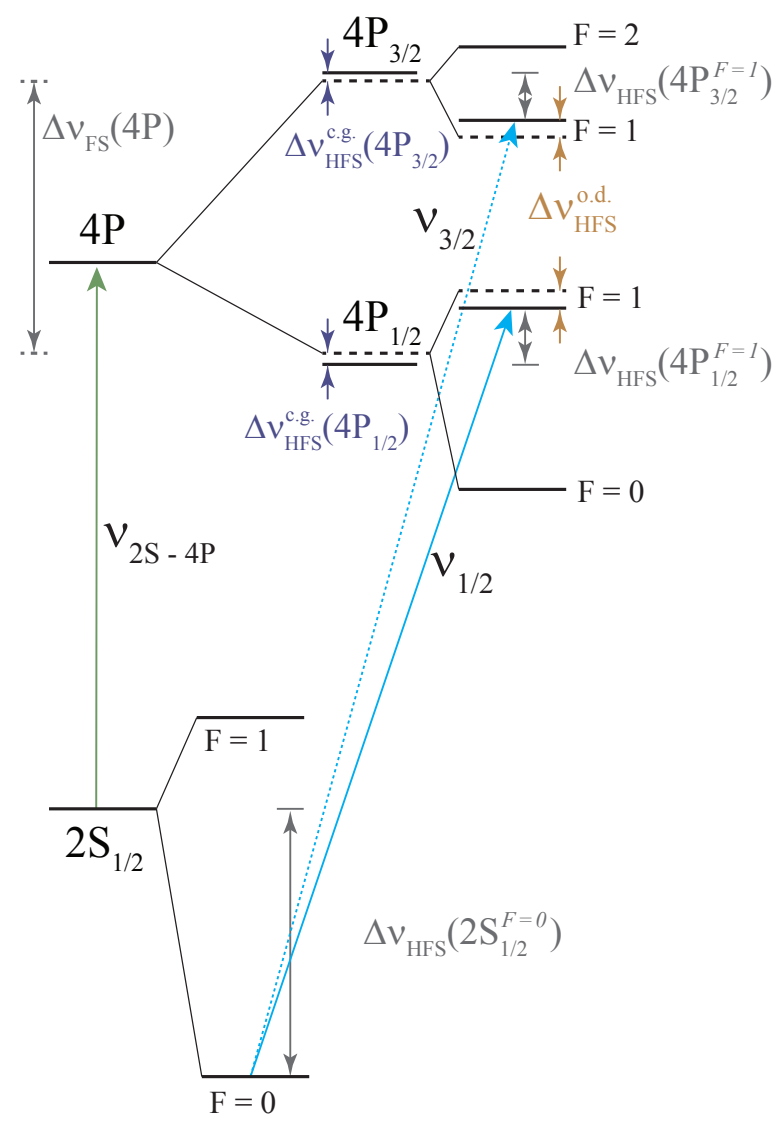

Figure 7.6.: Relevant fine and hyperfine components for hydrogen 2S-4P spectroscopy (splittings not to scale). Off-diagonal elements in the hyperfine Hamiltonian lead to shifts $\Delta \nu_{\mathrm{HFS}}^{o . d}$ in the $4 \mathrm{P}_{1 / 2}^{F=1}$ and $4 \mathrm{P}_{3 / 2}^{F=1}$ energy levels (orange) and shifts of $\Delta \nu_{\mathrm{HFS}}^{c . g}$ in the hyperfine centroids (violet). Dashed lines indicate the position of the energy levels without off-diagonal corrections. The transition frequency $\nu_{2 S-4 P}$ from the $2 S$ hyperfine structure centroid to the $4 \mathrm{P}$ fine structure centroid is determined from the measured transition frequencies $\nu_{1 / 2}$ and $\nu_{3 / 2}$.

\subsubsection{Hyperfine corrections}

In order to obtain the transition frequency from the $2 \mathrm{~S}$ hyperfine centroid to the $4 \mathrm{P}$ fine structure centroid, the measured transition frequencies $\nu_{1 / 2}$ and $\nu_{3 / 2}$ have to be corrected for the hyperfine shift of the $2 \mathrm{~S}_{1 / 2}^{F=0}$, the $4 \mathrm{P}_{1 / 2}^{F=1}$ and the $4 \mathrm{P}_{3 / 2}^{F=1}$ states (Tab. II in[45]):

$$
\begin{aligned}
\Delta \nu_{\mathrm{HFS}}\left(2 \mathrm{~S}_{1 / 2}^{F=0}\right) & =-133167.6257(51) \mathrm{kHz} \\
\Delta \nu_{\mathrm{HFS}}\left(4 \mathrm{P}_{1 / 2}^{F=1}\right) & =+1848.8(1) \mathrm{kHz} \\
\Delta \nu_{\mathrm{HFS}}\left(4 \mathrm{P}_{3 / 2}^{F=1}\right) & =-1847.7(1) \mathrm{kHz}
\end{aligned}
$$

These shifts have been obtained experimentally (for 2S, see[65]) and by extrapolation to higher $n$ and include a small off-diagonal term of $\Delta \nu_{\mathrm{HFS}}^{o . d .}= \pm 0.313 \mathrm{kHz}$ for the $4 \mathrm{P}$ states. The transition frequency from the $2 \mathrm{~S}$ hyperfine centroid to the $4 \mathrm{P}$ fine structure centroid $\left(\nu_{2 S-4 P}\right.$, Eq. (9) in the main text) is obtained by a weighted average of the hyperfine centroids and including the recoil correction in Eq. (7.11):

$$
\nu_{2 S-4 P}=\frac{1}{3}\left(\nu_{1 / 2}+2 \nu_{3 / 2}\right)-132552.092(75) \mathrm{kHz}-837.23 \mathrm{kHz} .
$$

The fine structure splitting $\Delta \nu_{F S}^{\text {theo }}(4 \mathrm{P})$ of the $4 \mathrm{P}_{1 / 2}^{F=1}$ and $4 \mathrm{P}_{3 / 2}^{F=1}$ states may readily 
be obtained from the difference in the total binding energies of the $4 \mathrm{P}_{1 / 2}^{F=1}$ and $4 \mathrm{P}_{3 / 2}^{F=1}$ states given in Tab. IV in [45]:

$$
\Delta \nu_{F S}^{\text {theo }}(4 \mathrm{P})=1367433.3(3) \mathrm{kHz} \text {. }
$$




\section{Rydberg constant and proton charge radius}

\subsection{Determination from hydrogen $2 \mathrm{~S}-4 \mathrm{P}$ and $1 \mathrm{~S}-2 \mathrm{~S}$}

The absolute transition frequency $\nu_{2 S-4 P}$ in Eq. (7.3) is used in combination with measurement of the $1 \mathrm{~S}-2 \mathrm{~S}$ transition frequency $[6,7]$ to extract the Rydberg constant and the proton charge radius via Eq. (1.2). A full summary of the theory used can be found in [4].

The code for the extraction has been validated by calculating several transition frequencies with arbitrarily accurate pseudo input data. Results have then been compared with the output of P. Mohr's code which is used for the global adjustment of fundamental constants in [4]. The values of $R_{\infty}$ and $r_{\mathrm{p}}$ determined using $\nu_{2 S-4 P}$ in Eq. (7.3) are:

$$
\begin{aligned}
R_{\infty} & =10973731.568037(91) \mathrm{m}^{-1} \\
r_{p} & =0.8293(91) \mathrm{fm} .
\end{aligned}
$$

These values are as accurate as the ones that can be extracted from a least squares adjustment of the aggregate world data of all precision electronic hydrogen spectroscopy (15 individual measurements) shown in Fig. 1.3 and Fig. 8.1 (see adjustment \# 8 in Tab. XXXVIII of [4]). Including the world data of precision electronic deuterium spectroscopy (consisting of 6 more measurements) in this adjustment, values of $R_{\infty}$ and $r_{\mathrm{p}}$ are obtained which are $16 \%$ more accurate than the ones presented in this work (see adjustment \# 6 in Tab. XXXVIII of [4]). The results obtained here are in agreement with the ones derived from laser spectroscopy of muonic hydrogen $[1,2]$, while there is a discrepancy of 3.8 combined standard deviations to the least squares adjustment of the previous electronic hydrogen data.

\subsection{Conclusions and Outlook}

\subsubsection{Hydrogen 2S-4P}

During this thesis, an experimental scheme, the corresponding hardware and theoretical background have been developed that enable high resolution spectroscopy of $2 \mathrm{~S}-n P$ transitions in atomic hydrogen with an uncertainty in the low kilohertz regime. The scheme has been applied to spectroscopy of the $2 \mathrm{~S}-4 \mathrm{P}$ transition first 
and the measurement serves as a raw model for future experiments of this kind (see below).

The first order Doppler shift is the leading order systematic shift for the spectroscopy of dipole allowed $2 \mathrm{~S}-n P$ transitions. One of the key components enabling measurements with the desired low kilohertz uncertainties is the development and characterization of an active fiber-based retroreflector (AFR, Sec. 3.5). It provides high quality anti-parallel phase-retracing laser beams and thereby a sufficiently large suppression of the first order Doppler effect (Sec.4).

The second major systematic effect, line shape distortions due to quantum interference of neighboring atomic resonances, has been studied experimentally using a large solid angle XUV split detector. In a differential measurement, apparent line shifts of up to $\pm 51 \mathrm{kHz}$ have been observed and represent the first direct demonstration of the underlaying effects in precision spectroscopy of resonances separated by more than one hundred line widths.

The apparent shifts observed in the $2 \mathrm{~S}-4 \mathrm{P}$ experiment are much larger than the proton size puzzle and can be brought under control by a suitable fit function (Sec. 2.2.1). Before this work, the common way of dealing with these effects has been to perform numerical simulations and correct the experimental data. Conceptional drawbacks of this approach, such as its high demand of computation power as well as the requirement of accurate input of the specific experimental realization, can be avoided using this proper fit function. Similar simulations, however, have also been performed for the case of hydrogen $2 \mathrm{~S}-4 \mathrm{P}$ in order to gain a general understanding of the underlying effects, to identify critical parameters in the experiment and to validate the robustness of the applied the line shape model.

Making use of this fit function, the absolute transition frequency from the $2 \mathrm{~S}$ hyperfine centroid to the $4 \mathrm{P}$ fine structure centroid has been determined:

$$
\nu_{2 S-4 P}=616520931625.8(2.2) \mathrm{kHz} \text {. }
$$

Confidence in this value and the derived values of the Rydberg constant $R_{\infty}$ and proton charge radius $r_{\mathrm{p}}$ is mediated by a number of conceptual advantages of the $2 \mathrm{~S}-4 \mathrm{P}$ experiment and various consistency checks that have been performed in this work (see below).

Examining Tab. 7.1, it is worthwhile to note, that besides the usual recoil, fine and hyperfine structure corrections, no further corrections have to be applied to the experimental data to account for systematic shifts in the line centers. This is mostly due to the well controlled and state selective excitation of the $2 \mathrm{~S}_{1 / 2}^{F=0}$ state by Doppler-free two photon excitation (Sec. 3).

In addition to the data analysis presented in the main body of the text, four more evaluations of the same experimental data have been performed and confirm the presented values. First, an independent and redundant data evaluation has been performed, starting from the raw data and repeating all necessary steps in the analysis. Second, the data has been analyzed using Voigt fits and the cancellation of 


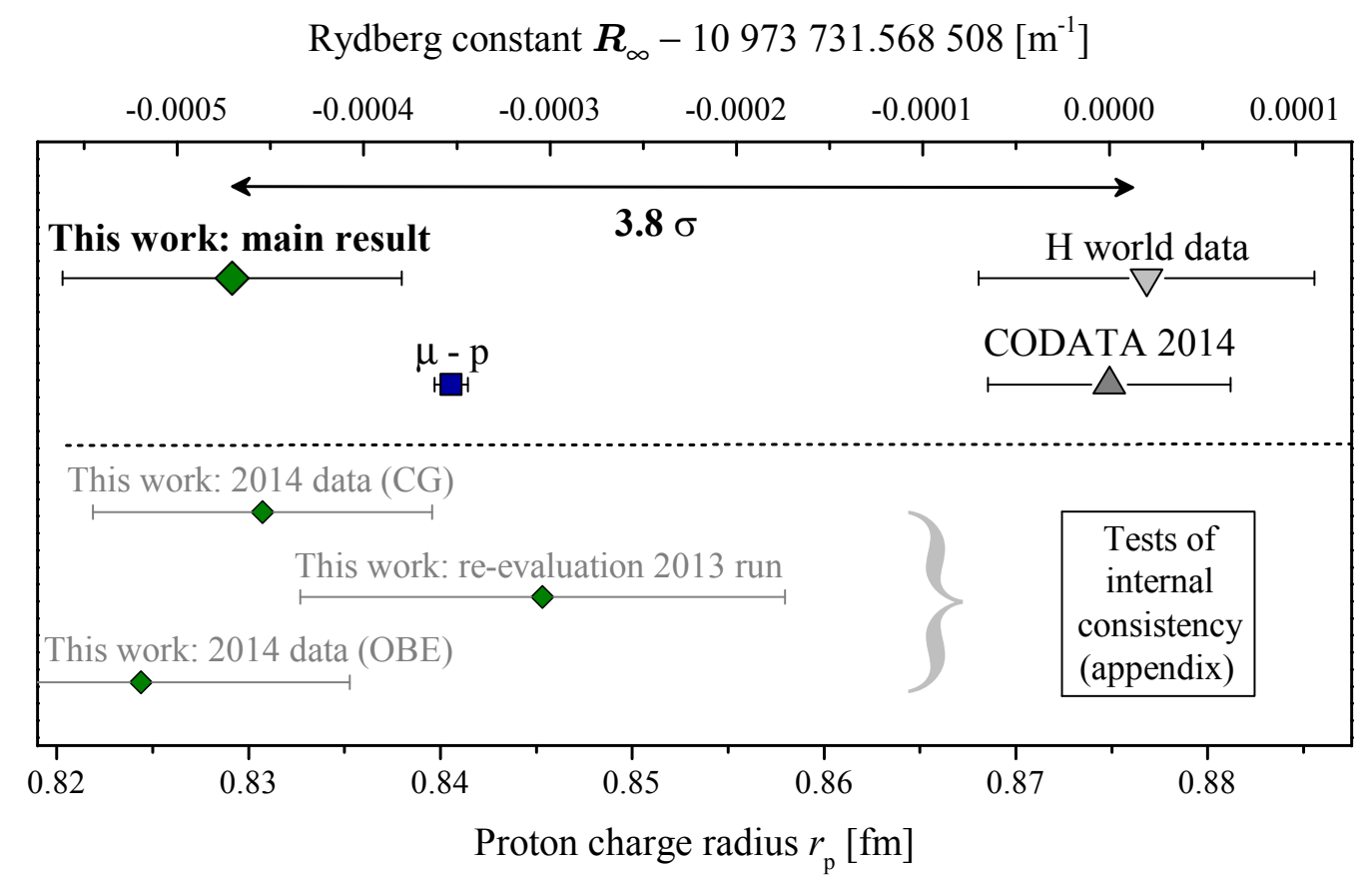

Figure 8.1.: Determinations of the Rydberg constant $R_{\infty}$ and the proton charge radius $r_{\mathrm{p}}$ (similar to Fig. 1.3). Values $R_{\infty}=10973731.568037(91) \mathrm{m}^{-1}$ and $r_{p}=0.8293(91) \mathrm{fm}$ derived from this work (green diamond) and spectroscopy of muonic hydrogen (blue square) agree. A discrepancy of 3.8 combined standard deviations between this work and the world data of precision hydrogen $(\mathrm{H})$ spectroscopy (light gray point) as well as the global adjustment of fundamental constants (gray triangle, CODATA 2014) is found. Tests for the internal consistency of the analysis in the main body of this thesis are given in App. A and confirm the presented results (see text for details).

QI line shifts in the determination of the $4 \mathrm{P}$ fine structure centroid (Sec. 2.4.3) has been utilized (Fig. 8.1, 2014 data CG, App. A.2). Third, previously unpublished data from an earlier measurement campaign in 2013 has been re-evaluated using the line shape model in Sec. 2.2.1 (Fig. 8.1, re-evaluation 2013 run, App. A.1). Fourth, the QI line shifts have been simulated numerically using the master-equation approach and the Voigt fit data have been corrected correspondingly (Fig. 8.1, 2014 data (OBE), App. A.3).

Moreover, excellent agreement has been found between the measured $4 \mathrm{P}$ fine structure splitting:

$$
\Delta \nu_{F S}^{\exp }(4 \mathrm{P})=1367432.7(2.8) \mathrm{kHz}
$$

and the accurate theoretical value of $\Delta \nu_{F S}^{\text {theo }}(4 \mathrm{P})=1367433.3(3) \mathrm{kHz} . \Delta \nu_{F S}^{\exp }(4 \mathrm{P})$ represents the most accurate experimental determination of a fine structure splitting in atomic hydrogen.

Various systematic shifts, such as the line shifts due to quantum interference, the 
Stark and Zeeman shift, affect the $4 \mathrm{P}_{1 / 2}^{F=1}$ and $4 \mathrm{P}_{3 / 2}^{F=1}$ states differently. Therefore, the measurement of the $4 \mathrm{P}$ fine structure splitting represents another stringent test of the internal consistency of the analysis.

\subsubsection{Future prospects}

In summary, using the two experiments leading to the most accurate determination of $R_{\infty}$ and $r_{\mathrm{p}}$ from electronic hydrogen to date, namely the combination of the $1 \mathrm{~S}-2 \mathrm{~S}$ transition $[6,7]$ and the $2 \mathrm{~S}-4 \mathrm{P}$ transition presented in this thesis, results in a consistent picture with the values obtained from muonic hydrogen. However, these consistent values of the proton charge radius and the Rydberg constant from muonic hydrogen differ by seven standard deviations from the ones quoted in the CODATA 2010 report [4].

The systematic effects dominating in this work are decoupled from the ones in the previous most accurate hydrogen experiments. This work, however, has shown that controlling systematic shifts at the level of the proton size puzzle is a difficult task even for the well controlled system using optical excitation for the population of the $2 \mathrm{~S}$ state.

The previous most accurate determinations of $R_{\infty}$ from hydrogen are exclusively based on measurements of two photon transitions, in particular the 2S-8S/D and 2S-12S/D transitions. Repeating these measurements with improved accuracy might point the way towards potential systematic effects that may yet be unknown in these experiments, similar to the line shape distortions due to quantum interference in this work (Sec. 2 and Sec. 6).

A number of new experiments are currently being planned and conducted and aim to contribute to the solution of the proton size puzzle:

E. A. Hessels group in Toronto is working towards an improved version of the historical Lamb shift experiment, i.e. measuring the energy difference of the $2 \mathrm{~S}$ and $2 \mathrm{P}$ state in atomic hydrogen. From such a direct measurement of the $2 \mathrm{~S}-2 \mathrm{P}$ energy difference with sufficient accuracy, an accurate value of $r_{\mathrm{p}}$ can be extracted without the need of relying on the 12 digits of accuracy of $R_{\infty}$.

Measurements of the 1S-3S absolute transition frequency in Paris (continuous wave excitation [23]) and Garching (direct frequency comb spectroscopy [24]) are underway and may provide complementary data input to the measurement of the $1 \mathrm{~S}-2 \mathrm{~S}$ transition frequency which serves as a corner stone in the extraction of $R_{\infty}$ and $r_{\mathrm{p}}$ from electronic hydrogen.

Meanwhile, the CREMA collaboration performed measurements of the 2S-2P energy differences in muonic deuterium and helium $\left(\mu-\mathrm{D}, \mu-{ }^{3} \mathrm{He}\right.$ and $\left.\mu-{ }^{4} \mathrm{He}\right)$. A new value of the deuteron charge radius may be compared to both, the proton-deuteron charge radius difference extracted from the $1 \mathrm{~S}-2 \mathrm{~S}$ isotope shift [66] as well as the deuteron radius derived from the combination of the electronic proton radius and the isotope shift. The comparison of $\mu$-He to results from elastic electron-heliumscattering as well as future measurements of the $1 \mathrm{~S}-2 \mathrm{~S}$ transition in $\mathrm{He}^{+}$which are 


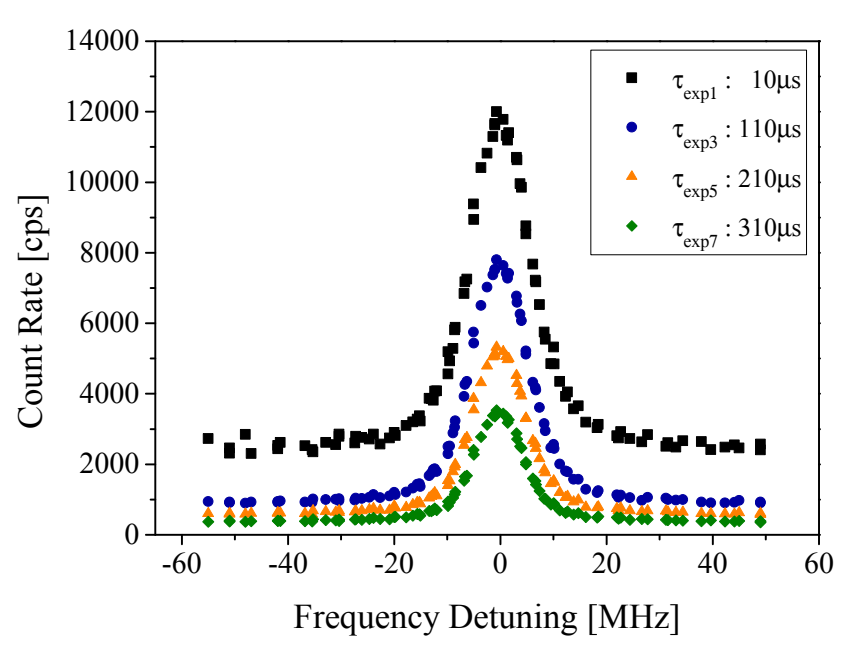

Figure 8.2.: Line scan sample data for the time-of-flight resolved detection of the $2 \mathrm{~S}-6 \mathrm{P}$ transition (similar to Fig. 3.3). A first signal from the 2S-6P transition has been obtained in February 2014. Depicted are four out of ten line profiles which are recorded simultaneously for different delay times $\tau_{j}$ between switching off the supply of $2 \mathrm{~S}$ atoms and their arrival at the 2S-6P interaction point. The data has been recorded in the same setup that was used for $2 \mathrm{~S}-4 \mathrm{P}$ spectroscopy.

in preparation in Garching and Amsterdam can test the puzzle at $\mathrm{Z}=2$.

Spectroscopy of the exotic positronium and muonium atoms is in preparation at ETH Zurich and at the Paul Scherrer Institute (PSI) in Villingen. These purely leptonic systems are free from finite size effects and feature significantly different sensitivities to certain QED corrections, e.g. relativistic recoil corrections in the case of positronium.

A number of new scattering experiments are also aiming to contibute to the solution of the puzzle. The MUSE experiment at PSI is scheduled for 2017. Scattering muons and electrons simultaneously from the same target may provide new data input for the discussion on proposed muon-specific forces (for a review of different proposals to resolve the proton size puzzle see [3]). Measurements of electromagnetic form factors at low momentum transfer are planned in Mainz, using the new MESA accelerator, and at Jefferson Lab (PRad). These low energy experiments may give important insight into the extrapolations to zero momentum transfer which are required to extract $r_{p}$ from existing form factor measurements at larger momentum transfer.

The methods developed in this work can be applied to higher lying $2 \mathrm{~S}-n \mathrm{P}$ transitions. In collaboration with the Garching 1S-3S team, an external cavity diode laser system similar to the $1 \mathrm{~S}-2 \mathrm{~S}$ and $2 \mathrm{~S}-4 \mathrm{P}$ systems has been built during this thesis. The system is used as a transfer reference for the measurement of the 1S-3S transition frequency in atomic hydrogen as well as for the spectroscopy of the 2S-6P transition. It is fully operational and first resonances of the $2 \mathrm{~S}-6 \mathrm{P}$ transition have been acquired in test measurements (Fig. 8.2).

Preparations for a measurement of the $2 \mathrm{~S}-6 \mathrm{P}$ absolute transition frequency are underway. Such a measurement features a number of interesting prospects for future improvement of the Rydberg constant utilizing the knowledge gained in this work.

First, the apparent line shifts due to quantum interference are now under control and the use of a split detector as discussed in Sec. 3.2 may no longer be considered 
mandatory. Instead, detectors may be placed above and below the interaction point, as was the case in earlier stages of the experiment (see Fig. E.4). This increases the overall detection solid angle coverage and improves the statistical uncertainty in the experiment. In addition to that, the efficiency of the photo electron collection may be increased by a more sophisticated electric field configuration in the detector volume.

Second, in spectroscopy of the $2 \mathrm{~S}-6 \mathrm{P}$ transition, the relative weights of certain systematic effects will be different compared to the $2 \mathrm{~S}-4 \mathrm{P}$ transition. The sensitivity to external fields increases, while the influence of the quantum interference line distortions on the natural line shape decreases due to its proportionality to the natural line width. The latter is $3.8 \mathrm{MHz}$, i.e. smaller by a factor of roughly three compared to the $2 \mathrm{~S}-4 \mathrm{P}$ transition. The atomic beam divergence currently dominates the observed line width of about $10 \mathrm{MHz}$ in the $2 \mathrm{~S}-6 \mathrm{P}$ data. Approaching the natural line width by further restricting the atomic divergence angle will lead to multiple benefits: a smaller influence of the line shape distortions due to quantum interference and improved uncertainties in the determined line centers for each individual line scan. The latter is of particular importance for limiting the first order Doppler effect by comparing results from sub-samples with different mean velocity.

Third: a differential measurement of the $6 \mathrm{P}$ fine structure splitting may be performed by rapid alternation between excitation of the $6 \mathrm{P}_{1 / 2}^{F=1}$ and $6 \mathrm{P}_{3 / 2}^{F=1}$ states similar to the polarization switching used for the study of quantum interference line shifts in Sec. 3.3. Such a differential measurement with a switching time of around one second will be insensitive to the common Doppler shift to a high degree and thereby improves the sensitivity to other systematic shifts, e.g. the Zeeman and Stark effect or the line shape distortions due to quantum interference.

Fourth: measurements at higher temperatures of the hydrogen nozzle have been performed at different occasions during this thesis. At a nozzle temperature of $30 \mathrm{~K}$, the overall signal rates decrease by a factor of about three compared to $5.8 \mathrm{~K}$, however the nozzle does not get blocked with time (60 min to 90 min time scale at $5.8 \mathrm{~K}$ ) and the experiment can be run continuously. Also, evaporating the frozen hydrogen gas from the nozzle is avoided, which is one of the main sources of damage to the XUV detectors and the degradation of the $243 \mathrm{~nm}$ enhancement cavity mirrors for the $1 \mathrm{~S}-2 \mathrm{~S}$ excitation. Combined with an improved detection efficiency of the XUV detectors, measurements at $30 \mathrm{~K}$ may also contribute to an improved upper limit for the residual uncompensated first order Doppler effect by contributing data with larger atom mean velocities.

As discussed in Sec. 1.4, an overdetermined system is required to test the internal consistency of QED, i.e. at least three independent transition frequency measurements are required for a test of QED in atomic hydrogen. Comparing the results from this work with upcoming new experiments, such as the spectroscopy of the 2S-6P transition or one of the experiments named above, will certainly bring new insight into the proton size puzzle.

Furthermore, the uncertainty of the muonic hydrogen value of $r_{\mathrm{p}}$ is small enough 
to enable a test of QED in atomic hydrogen that is limited by equal contributions from experimental input and yet un-calculated higher order QED terms. Measurements of the Rydberg constant with improved accuracy may therefore also be expected to fuel further developments in the QED theory of the hydrogen atom. 



\section{Appendix}

\section{A. Data analysis: Tests of internal consistency}

\section{A.1. Re-evaluation of 2013 measurement run}

The detailed analysis of line shape distortions due to quantum interference of neighboring atomic resonances discussed in the main body of this thesis has been triggered by experimental observations in a measurement run in 2013. In this measurement run, the polarization switching scheme and the PM fiber 1 in Fig. 3.4 were not yet implemented. Instead, the input polarization to a non-PM single mode fiber in the place of PM fiber 1 was defined by an optical isolator. Thereby, the polarization at the 2S-4P interaction point (Fig. 3.1 and Fig. 3.2) was not well controlled. While the polarization output of non-PM single mode fiber is not necessarily linear, it is reproducible to some certain extent, if the working conditions (temperature, mechanical stress and strain, etc.) do not change significantly.

Despite this poor polarization control in the 2013 setup, significant differences in the extracted transition frequency of the $2 \mathrm{~S}_{1 / 2}^{F=0}$ to $4 \mathrm{P}_{1 / 2}^{F=1}$ transition could be observed as a function of the input polarization to the single mode fiber. Line centers extracted from usual Voigt fits of the data are depicted on the left side of Fig. A.1. The results from three data blocks $\left[\mathrm{H}, \mathrm{V}, 45^{\circ}\right]$ are compared. The input polarization to the single mode fiber was adjusted to be horizontal $(\mathrm{H})$, vertical $(\mathrm{V})$ and at an angle of $45^{\circ}$ with respect to the to the optical table. The differences observed in the mean values are significant and exceed $10 \mathrm{kHz}$. This is larger than the proton size puzzle of $8.9 \mathrm{kHz}$ and the reason why the data was previously unpublished.

The 2013 data was re-analyzed after the detailed study of the quantum interference line shape distortions. Using Eq. (2.8) as a fit function instead of the Voigt profile removes the excessive scatter from the data and the results of the different data blocks agree (Fig. A.1, right). The total data set, acquired over three weeks of run time, consists of 18390 (6 850) resonances for the $2 \mathrm{~S}_{1 / 2}^{F=0} \rightarrow 4 \mathrm{P}_{1 / 2}^{F=1}\left(2 \mathrm{~S}_{1 / 2}^{F=0} \rightarrow 4 \mathrm{P}_{3 / 2}^{F=1}\right)$ transition. When combining this data with the measurement of the 1S-2S transition frequency (analogous to the discussion in Sec. 7), the values for the Rydberg constant and proton size: 

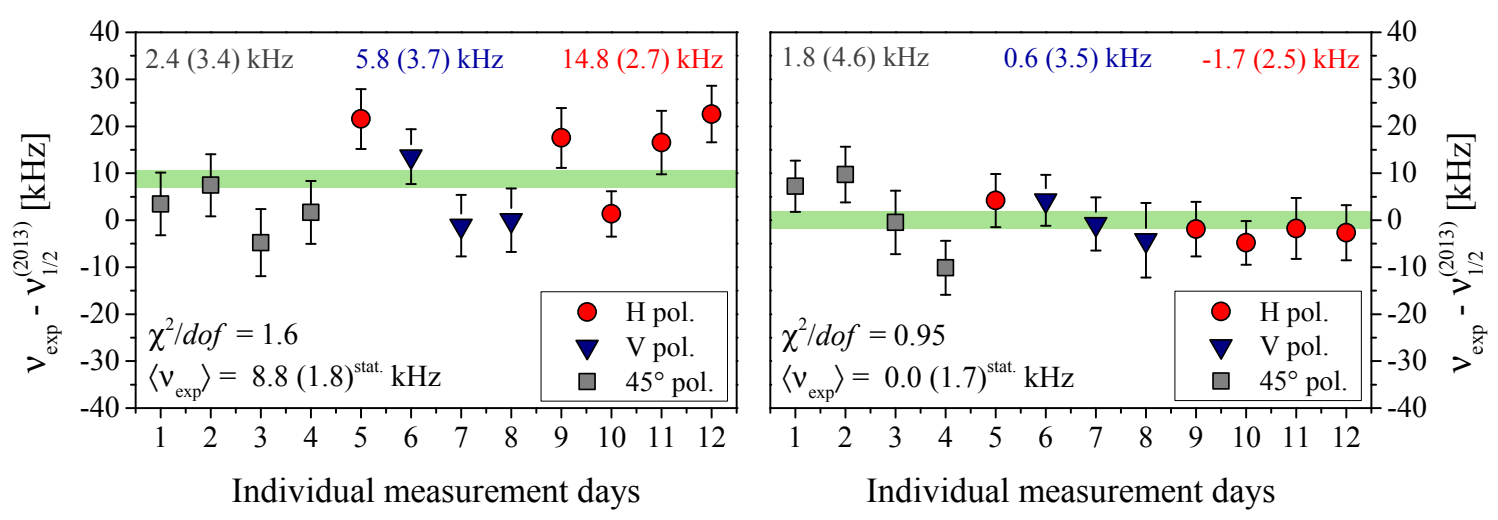

Figure A.1.: Re-evaluation of $2 \mathrm{~S}_{1 / 2}^{F=0}-4 \mathrm{P}_{1 / 2}^{F=1}\left(\nu_{1 / 2}\right)$ data from a previous measurement campaign in 2013. Left: Significant differences between different data blocks [H, V, 45], corresponding to different input polarizations (see text for details), are observed using usual Voigt fits to the data. Data block averages are given in colored numbers above the data. Right: Same data, but line distortions due to quantum interference are taken into account by using Eq. (2.8) for data analysis. The scatter is reduced and results from different data blocks agree. Note the $+8.8 \mathrm{kHz}$ offset obtained by improper modeling of the data.

$$
\begin{aligned}
R_{\infty}^{(2013)} & =10973731.56820(13) \mathrm{m}^{-1} \\
r_{p}^{(2013)} & =0.846(13) \mathrm{fm} \\
\Delta \nu_{F S}^{\exp (2013)}-\Delta \nu_{F S}^{\text {theo }} & =2.6(3.8) \mathrm{kHz}
\end{aligned}
$$

are found in agreement with the ones from the 2014 run (Sec. 8). The same is true for the extracted value of the $4 \mathrm{P}$ fine structure splitting $\Delta \nu_{F S}^{\exp }$ (2013).

As the entire apparatus has been disassembled and components have been upgraded between the two runs, this finding does not only give a strong indication for the internal consistency of the data analysis, but also represents a strong exclusion threshold for potential uncontrolled systematic effects in the experiment.

The re-analysis of the 2013 data also nicely demonstrates the strength of the expanded line shape model. The polarization state of the excitation light at the $2 \mathrm{~S}-4 \mathrm{P}$ interaction point is completely unknown in the case of the 2013 data. Furthermore, it is certainly not reproducible in studies much later than the experiment was performed. A numerical simulation of the QI shifts is therefore impossible. Still, the effect can be taken into account by the model function and is efficiently canceled. 


\section{A.2. Center of weight method for 2014 data}

In Sec. 2.4.3, the possibility has been discussed that apparent geometry dependent QI line shifts may be canceled by a weighted average of the two transitions from the $2 \mathrm{~S}_{1 / 2}^{F=0}$ to the $4 \mathrm{P}_{1 / 2}^{F=1}$ and $4 \mathrm{P}_{3 / 2}^{F=1}$ states (Eq. (2.30)). The following procedure is applied to the $2 \mathrm{~S}-4 \mathrm{P}$ absolute frequency data to do so:

- the experimental data is fit with a Voigt profile, yielding the average values depicted in Fig. B.1

- a weighted average of the two detectors is performed for $4 \mathrm{P}_{1 / 2}$ and $4 \mathrm{P}_{3 / 2}$ independently using the relative line strengths depicted in Fig. B.2 (weights are 1 for $4 \mathrm{P}_{1 / 2}$ and depend on $\theta_{L}$ for $4 \mathrm{P}_{3 / 2}$ ).

- subsequently, a weighted average over all linear laser polarization angles $\theta_{L}$ is performed for $4 \mathrm{P}_{1 / 2}$ and $4 \mathrm{P}_{3 / 2}$ independently, respecting the remaining $\theta_{L^{-}}$ dependence of the line strengths.

- finally, the fine structure centroid is determined by averaging the absolute frequency values of the transitions frequencies from the $2 \mathrm{~S}_{1 / 2}^{F=0}$ to the $4 \mathrm{P}_{1 / 2}^{F=1}$ and $4 \mathrm{P}_{3 / 2}^{F=1}$ states, respectively, with weights of $1: 2$.

The weights for averaging (line strength ratios) can be taken from the experiment and at the level of accuracy required $(\approx 5 \%)$ they are identical to the ones obtained by the OBE simulation as shown in Fig. B.2. The uncertainty for the residual shift due to quantum interference in this analysis was estimated using both the masterequation approach and the perturbative approach and was found to be less than $500 \mathrm{~Hz}$. The extracted values of $R_{\infty}$ and $r_{\mathrm{p}}$ agree with the ones in the main body of this thesis and given by:

$$
\begin{aligned}
R_{\infty}^{(\mathrm{CG})} & =10973731.568054(91) \mathrm{m}^{-1} \\
r_{p}^{(\mathrm{CG})} & =0.8309(91) \mathrm{fm}
\end{aligned}
$$

The center of weight method serves as another indicator for internal consistency. It is, however, conceptually weaker than the fit of the expanded line shape. Line strengths have either to be measured as a function of the linear laser polarization angle $\theta_{L}$ or the have to be extracted from a numerical simulation. If the signal levels drift with time, a differential measurement at angles $\theta_{L}$ and $\theta_{L}+90^{\circ}$ is required. If the polarization is not linear and / or reproducible, this method highly probable to fail. 


\section{A.3. Correcting Voigt fits using master-equation approach (2014 data)}

The most difficult way of dealing with the QI line shifts is the correction of experimental data by simulation results as has been discussed before. Various effects may influence the modeling of the experimental implementation. A selection of effects studied for the $2 \mathrm{~S}-4 \mathrm{P}$ experiment are summarized in the next section. Due to its complexity and the various effects to consider, the overall uncertainty in the corrections is estimated to be $15 \%$.

The simulation results discussed in Sec 6.2 are used to correct for the inadequate treatment of QI line distortions by the Voigt fits (Sec.6.1.3). Once corrected, this data yields a $4 \mathrm{P}$ fine structure splitting that is in agreement with the theoretical prediction. In contrast to that, when ignoring the effects of quantum interference, the observed $4 \mathrm{P}$ fine structure splitting is found to be wrong by about $10 \mathrm{kHz}$. Values for $R_{\infty}$ and $r_{\mathrm{p}}$ determined from this analysis agree with the ones in the main body of the text (Sec.8). The additional uncertainty for the OBE corrections included in the analysis is $1.6 \mathrm{kHz}$.

$$
\begin{aligned}
R_{\infty}^{(\mathrm{OBE})} & =10973731.56799(11) \mathrm{m}^{-1} \\
r_{p}^{(\mathrm{OBE})} & =0.824(11) \mathrm{fm} \\
\Delta \nu_{F S}^{\exp (\mathrm{OBE})}-\Delta \nu_{F S}^{\text {theo }} & =-0.8(3.4) \mathrm{kHz} .
\end{aligned}
$$




\section{B. Systematic uncertainties in master-equation approach}

Using the split detector arrangement and the periodic polarization switching for the measurement of both fine structure components $4 \mathrm{P}_{1 / 2}$ and $4 \mathrm{P}_{3 / 2}$, six different data sets are obtained which need to be described simultaneously with identical input parameters in the OBE simulation (Sec 6.2). Those data sets are: QI line shifts for the individual detectors CEM $1 \& 2$ (amplitude and phase difference $\Delta \theta_{L}$, see Fig. B.1) and the relative line strengths observed upon $90^{\circ}$ rotation of the polarization direction (see Fig. B.2). These data sets can be used to put limitations on different systematic effects in the OBE description.

\section{B.1. Experimental restrictions}

\section{B.1.1. Observed line centers}

Fig. B.1 shows the line centers of $2 \mathrm{~S}-4 \mathrm{P}_{1 / 2}$ transition (top) and $2 \mathrm{~S}-4 \mathrm{P}_{3 / 2}$ transition (bottom) as a function of the linear laser polarization $\theta_{L}$ extracted from the individual split detectors CEM $1 \& 2$ as well as the corresponding OBE simulation. The phase shift $\Delta \theta_{L}$ between the two data sets is expected due to the different solid angles covered by the two detectors. A sine fit of the experimental data of $2 S-4 P_{1 / 2}$ results in an amplitude of about $\pm 34 \mathrm{kHz}$ for the individual detectors. The extracted $2 \mathrm{~S}-4 \mathrm{P}_{3 / 2}$ transition frequency varies by $\pm 15 \mathrm{kHz}$. The individual signals from the detectors CEM1\&2 are much more sensitive to changes in the geometry than their difference shown in Fig. 6.2. Correcting the individual detectors' data sets by the line shifts predicted by the OBE simulation and extracting the $4 \mathrm{P}$ fine structure splitting can be used for an independent test of the OBE model (Sec. A.3).

\section{B.1.2. Observed line strengths}

The line strength ratios for the $2 \mathrm{~S}-4 \mathrm{P}_{1 / 2}$ and $2 \mathrm{~S}-4 \mathrm{P}_{3 / 2}$ transitions as a function of laser polarization are shown in Fig. B.2 and are obtained as follows: each line scan consists of two individual resonances corresponding to laser polarisation angles $\theta_{L}$ and $\theta_{L}+90^{\circ}$ for each individual detector. The polarization of the excitation light rotated by $90^{\circ}$ every second while the alignment inside the vacuum chamber does not change. Individual relative line strengths are very well comparable because changes in the system, such as the flux of hydrogen $2 \mathrm{~S}$ atoms, $243 \mathrm{~nm}$ power or the gain of the detectors varies on much longer time scales. Only the fact that the $486 \mathrm{~nm}$ 

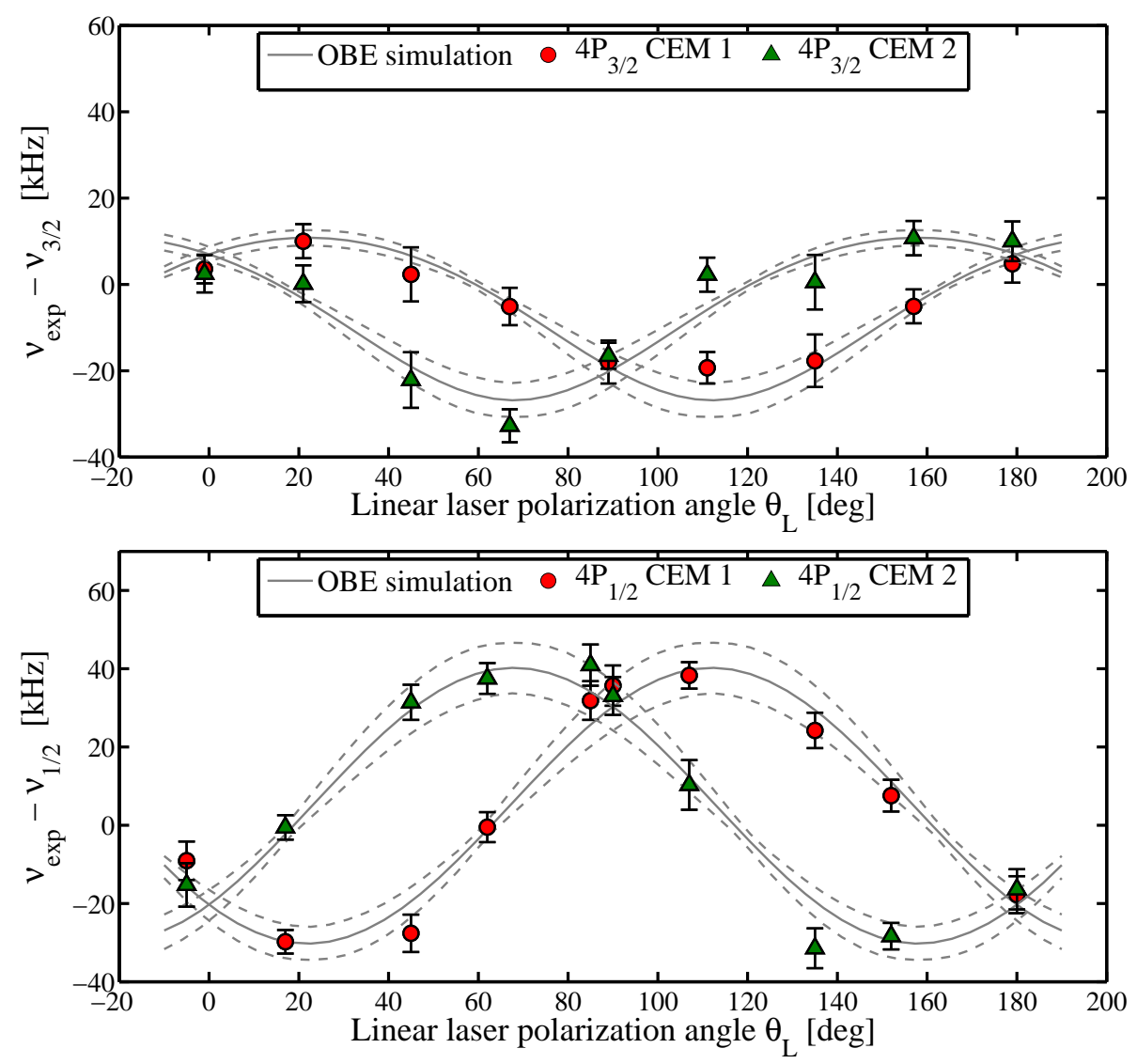

Figure B.1.: Line centers extracted using Voigt fits of the individual detectors' data (CEM 1\&2) as a function of laser polarization angle $\theta_{L}$. Top: $2 \mathrm{~S}-4 \mathrm{P}_{3 / 2}$ transition. Bottom: $2 \mathrm{~S}-4 \mathrm{P}_{1 / 2}$ transition. The observed apparent variations of the line center positions of the individual detectors due to quantum interference are $\pm 15 \mathrm{kHz}( \pm 34 \mathrm{kHz})$ for the $2 \mathrm{~S}-4 \mathrm{P}_{3 / 2}\left(2 \mathrm{~S}-4 \mathrm{P}_{1 / 2}\right)$ transition. The frequency axes are offset by the transition frequencies $\nu_{1 / 2}$ and $\nu_{3 / 2}$ in Eq. (7.1) and Eq. (7.2).

laser power might be slightly different for the two polarizations due to different fiber coupling efficiencies needs to be taken into account. For this, we record the signal of PD1, the photo diode that is used to monitor the amount of light coupled back into the PM fiber 1. For each setting, i.e. orientation of the fiber axes, the ratio between the line strengths observed at angles $\theta_{L}$ and $\theta_{L}+90^{\circ}$ is computed after correcting for the different power levels. The resulting ratios are shown in Fig. B.2.

No significant $\theta_{L}$-dependence of the line strength can be observed for the $2 \mathrm{~S}-4 \mathrm{P}_{1 / 2}$ transition, indicating equal detection probability for different polarizations. The small offset of a few parts per thousand of the measured line strength ratios from unity is most probably caused by a residual power imbalance between the two orthogonal polarization directions. The fact that the two individual detectors display almost identical line strength ratios at all polarization angles strengthens this argu- 


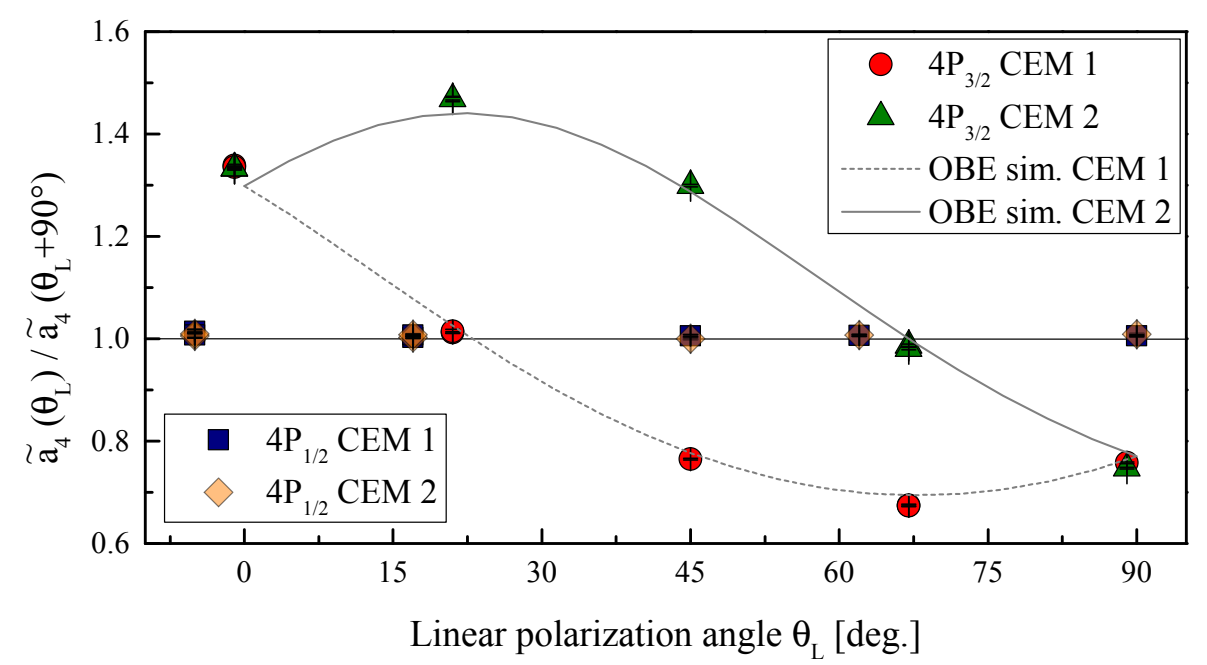

Figure B.2.: Observed line strengths of the $2 \mathrm{~S}-4 \mathrm{P}$ transitions. Line strengths $\tilde{a}_{4}$ at linear laser polarization angles $\theta_{L}$ and $\theta_{L}+90^{\circ}$ are obtained in a differential measurement as explained in Sec.6.1.1. The polarization angle is switched once per second. Thereby the influence of slowly drifting parameters, such as the flux of $2 \mathrm{~S}$ atoms, the 1S-2S excitation light power and the detector gains, on the ratio $\tilde{a}_{4}\left(\theta_{L}\right) / \tilde{a}_{4}\left(\theta_{L}+90^{\circ}\right)$ is reduced. For the isotropic from the $4 \mathrm{P}_{1 / 2}$ state, $\tilde{a}_{4}$ is independent of $\theta_{L}$ as expected (gray solid line, blue and orange squares). Due to the the anisotropic emission from the $4 \mathrm{P}_{3 / 2}$, the observed line strength is a function of $\theta_{L}$. The agreement of the experimental data with the OBE (Sec. 6.2) simulation is found to be better than $3 \%$ (gray dashed line for CEM1 and gray solid line for CEM2).

ment and confirms again a good reproducibility of the numerous detector parameters as has been indicated by other symmetries already.

In case of the $2 \mathrm{~S}-4 \mathrm{P}_{3 / 2}$ transition, the emission is not isotropic and depending on the exact detector geometry, variations in the observed lines strengths are to be expected as a function of laser polarization. Simulations of the expected line strength ratios for ideal split detectors with different detection opening angles are summarized in Fig. B.2 along with the ratios extracted from experimental data. Although there is no free parameter in the simulation of the detection efficiency (Sec. B.2.2) to fit the experimental data, the agreement with the latter is very good.

\section{B.1.3. Linear laser polarization angle}

The input polarizations to the PM fiber guiding the light to the spectroscopy region inside the vacuum chamber have been carefully aligned to coincide with the preferred fiber axes. The intensity extinction ratio has been measured to be better than 1:200 at the $2 \mathrm{~S}-4 \mathrm{P}$ interaction point for either axis. The PM fiber output that defines $\theta_{L}$ inside the vacuum chamber is fixed to a rotatable mount with vernier scale and readoff uncertainty of $\pm 0.04^{\circ}$. While relative changes of $\theta_{L}$ can be measured accurately, 
the angle with respect to the symmetry axis of the detector is hard to determine in the setup. Instead, we extract it from a sine fit of the $2 \mathrm{~S}-4 \mathrm{P}_{1 / 2}$ data shown in Fig. 6.2. The resulting uncertainty of the fit is $\delta \theta_{L}=1.5^{\circ}$. This uncertainty is included in the confidence bands shown for the OBE simulation in Fig. 6.2 and Fig. B.1.

\section{B.2. Detector acceptance and efficiency}

\section{B.2.1. Imperfections in detector geometry}

Talking about geometry dependent line shifts, the first class of systematic effects that come to mind are imperfections of the geometry itself. These imperfections include:

- differences in the detection opening angles covered by the detectors CEM 1\&2,

- a displacement of the interaction point

- and a inhomogeneous transmission of the spherical Faraday mesh separating the interaction region an the detectors.

These imperfections are mostly limited by the machining of the detector parts. In addition, the symmetry of experimental observations between the individual detectors on one hand and between observations at laser polarization angles $\theta_{L}$ and $\theta_{L}+90^{\circ}$ on the other hand are strong indicators for the symmetry and reproducibility of the setup (see Figs. 6.1 and B.2).

Imperfections of the geometry that could lead to a dependence of the detectors' quantum efficiency on the emission direction from the interaction point, such as the homogeneity and reproducibility of the graphite coating inside the detectors as well as the electric field configuration, are discussed separately further below.

Another geometric factor that could in principle reduce the contrast in the observed QI line shifts is the orientation of the separating wall between the detectors. In the limiting case of a separating wall in the $\mathrm{y}$-z-plane instead of the $\mathrm{x}$-z-plane, i.e. in the plane of polarization rotation instead of a plane perpendicular to it, the sensitivity to QI line shifts in the difference of the extracted line center positions CEM1 - CEM2 vanishes.

However, the individual detectors would still observe line shifts with reduced amplitude. At the same time, the phase difference $\Delta \phi$ decreases with increasing misalignment and reaches zero when the separating wall is aligned with the y-z-plane. We simulated this effect using the perturbative model and a large misalignment of $10^{\circ}$. In the range of detection opening angles from $25^{\circ}$ to $90^{\circ}$, the maximum reduction in contrast for this large misalignment is found to be only $1.5 \%$, i.e. less than $0.5 \mathrm{kHz}$. In the experiment, we expect a much smaller misalignment than $10^{\circ}$, so that this effect is negligibly small. 


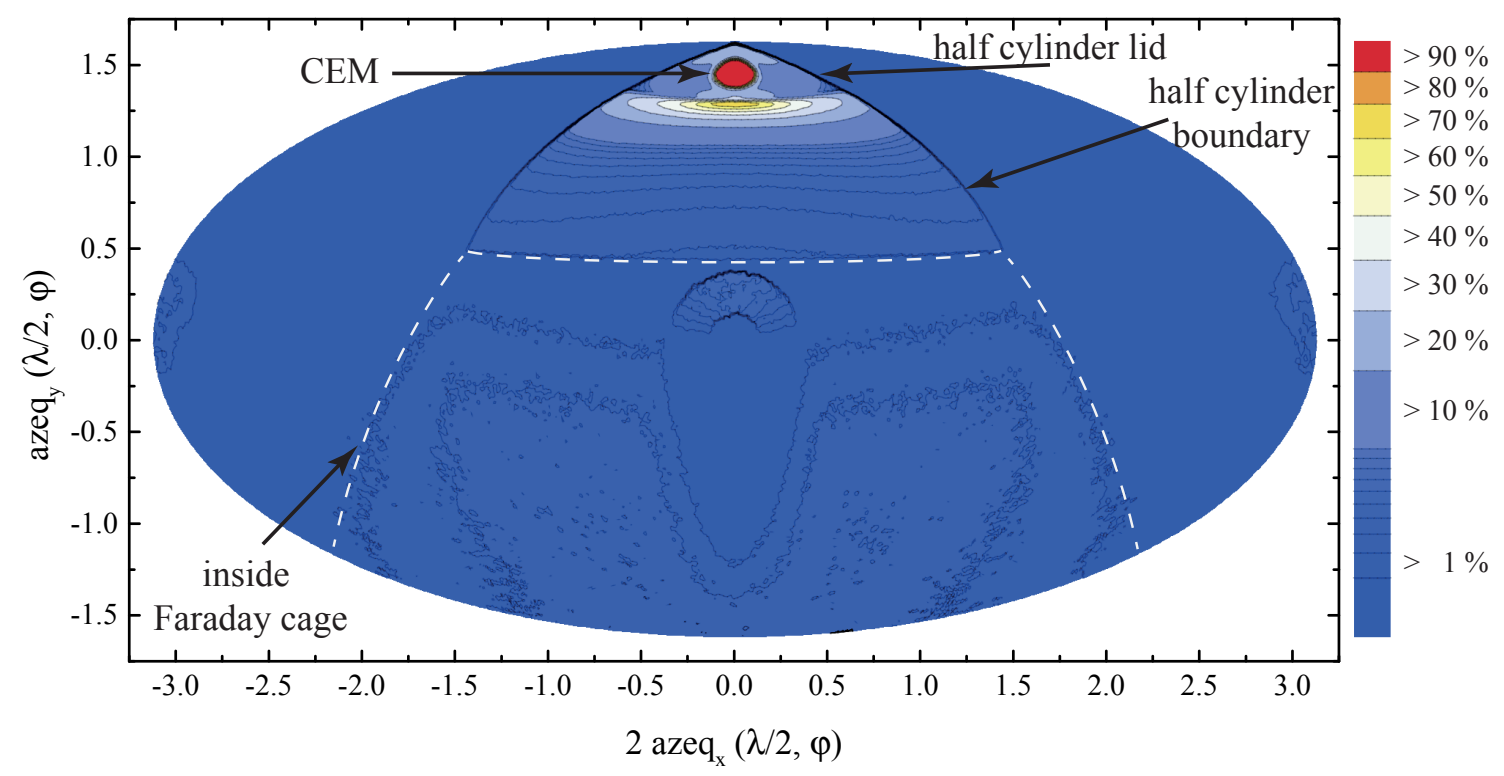

Figure B.3.: Map of local detection efficiency for one of the Lyman- $\gamma$ fluorescence split detectors (see also Fig. 3.2). The Aitoff projection, commonly known from projections of the globe, is used for illustration (Eq. (B.1) and Eq. (B.2)). The origin is located at the $2 \mathrm{~S}-4 \mathrm{P}$ interaction point and shown is the projection of a sphere around the origin. The contour plot indicates the simulated probability, that a photo emitted in a certain direction is detected by the channel electron multiplier CEM via a photo electron. Photons that fly to the second split detector (outside half cylinder boundary) are essentially lost with $100 \%$ probability. However, the map shows that a certain fraction of electrons originating from inside the Faraday cage in Fig. 3.2 (inside white dashed line) can also be detected. This is essential for the comparison of the OBE simulations and the experimental data in Sec. 6.2. azeq: equatorial azimuthal equidistant projection.

\section{B.2.2. Simulation of electric field configuration}

To fully recover the QI line shifts in the signals acquired by the XUV detectors in the OBE simulation discussed in Sec. 6.2, the detector sensitivity as a function of detection direction $\vec{r}_{d}(\theta, \phi)$ has to be taken into account. This sensitivity is predominantly given by the distribution of electric fields inside the detector volume. The detection efficiency is modeled by a 3D simulation, tracing photons from the spectroscopy region through the detector.

Photons in the simulation are converted into photo electrons when they impinge on the graphite coated inner walls of the detector. The photo electron energies are uniformly distributed between $0 \mathrm{eV}$ and $7.75 \mathrm{eV}$. The latter corresponds the energy of a Lyman- $\gamma$ photon minus the work function of graphite which is about $5 \mathrm{eV}$. The emission angles are distributed proportional to $\cos \beta$, where $\beta$ is the angle to the surface normal vector. This angular distribution is chosen due to the roughness of the graphite surface (see Fig $2 a$ in Ref. [67]). Electric fields which guide the electrons towards the CEM are computed setting the CEM input voltage to $+270 \mathrm{~V}$ and 
all other surfaces to ground potential. The photo-electron trajectories are traced through the electric fields. Photons are detected if their corresponding photo electrons reach the input surface of the CEM or if they hit the latter directly (red spot in Fig. B.3). If an electron hits the detector wall, it is lost with unity probability.

The resulting map of local detection efficiency is shown in Fig. B.3 and the influence of local detection efficiency on the observed QI line shifts is discussed in Sec. 6.2. For illustration, the Aitoff projection of a sphere around the 2S-4P interaction point is used. The projection is defined by [68]:

$$
\begin{aligned}
\operatorname{2azeq}_{\mathrm{x}}(\lambda / 2, \varphi) & =\frac{2 \cos (\varphi) \sin (\lambda / 2)}{\operatorname{sinc}(\alpha)} \\
\operatorname{azeq}_{\mathrm{y}} & =\frac{\sin (\varphi)}{\sin (\alpha)},
\end{aligned}
$$

where

$$
\alpha=\arccos (\cos (\varphi) \cos (\lambda / 2)),
$$

$\lambda$ denotes the longitude from the central meridian (y-z-plane in our coordinate system, Fig. 2.3) and $\varphi$ is the latitude [68] (measured from the equatorial plane, the $\mathrm{x}$-y-plane in our coordinate system).

It is important to note that our simulation of the detection efficiency includes a number of basic assumptions, such as the distributions of photo electron energies and and emission angles, however, there is no free parameter involved that is used to fit the experimental data sets presented in Sec. 6.

\section{B.2.3. Emission direction dependent quantum efficiency}

Assuming an ideal geometric arrangement of the experiment, additional modifications of the observed QI line shifts are possible, if the detectors' quantum efficiency depends on direction of detection $\hat{r}\left(\theta_{d}, \phi_{d}\right)$. Possible sources of such a dependence include the homogeneity and reproducibility of the graphite coatings on the detectors' inner walls which are used as photo cathodes.

Two ideal cases can be distinguished: pure dependence of the quantum efficiency as a function of $\theta_{d}$, i.e. the rotational symmetry around the z-axis is still intact, and the dependence on the angle $\phi_{d}$, which breaks the rotation symmetry. For the first case, we have argued in Sec. 2.4.3 that the size of the QI line shifts changes, while other important features, such as the position of the magic angle, remain the same. For a $\phi$ dependence of the detection efficiency, however, this is not the case. Depending on the specific realization of the $\phi$ dependence, the behavior may change drastically and a magic angle may not even exist any more.

We model the influence of the direction dependent quantum efficiency in our detectors for the OBE approach as discussed in Sec. 6.2. The simulated map of local detection efficiency is shown in Fig B.3. Dependencies on both angles $\theta_{d}$ and $\phi_{d}$ are observed in this simulation. The detection efficiency due to the electric field 
configuration ranges from $100 \%$ if photons hit the input facet of the CEMs directly down to less than $10 \%$ in regions far away from the CEM and at the edges of the detector.

The differences between subsequent layers of complexity in the simulations have been compared in Fig. 6.3. The difference in the predicted maximum line shifts with and without the direction dependent detection efficiency map included in the OBE simulation is $-7.9 \mathrm{kHz}$, i.e. $21 \%$ of the amplitude predicted by the full treatment. It is important to note again that while basic assumptions have to be put into the simulation, no free parameter is involved that is used to fit the simulation to the experimental data sets.

To test the stability of the obtained results, we have varied the basic assumptions put into the simulation of the detection efficiency map. In particular, the distribution of photo electron energies and emission angles as well as the probabilities for electron and photon reflection from the detector walls have been altered and we conclude that the correction due to the detector efficiency map should be accurate to $20 \%$ or better, i.e. $4 \%$ of the shift predicted by the full treatment, i.e. less than $1.6 \mathrm{kHz}$ out of $35 \mathrm{kHz}$ total shift.

\section{B.2.4. Emission polarization dependent quantum efficiency}

Up to this point, it has been assumed that the detection efficiency in a certain direction $\hat{r}\left(\theta_{d}, \phi_{d}\right)$ is identical for emission from the three spherical components. However, this does not necessarily have to be the case, e.g. if the efficiency of photo electron emission were to depend on the incoming electric field vector. The corresponding change in the expected line shifts is hard to model because precise knowledge about the processes involved is required. On the other hand, possible polarization dependent effects can tightly be constrained by the experimentally observed line strengths as a function of laser polarization.

Most useful for this purpose is the measured line strengths of the $2 \mathrm{~S}-4 \mathrm{P}_{1 / 2}$ transition in Fig. B. 2 because of the isotropic emission pattern of the $4 \mathrm{P}_{1 / 2} \rightarrow 1 \mathrm{~S}$ decay. Although the number of emitted photons is constant for all emission directions $\hat{r}\left(\theta_{d}, \phi_{d}\right)$, the relative contributions of the three spherical components changes according to their respective emission patterns. Accordingly, any polarization sensitivity of the detection efficiency should manifest itself as a modulation of the $2 \mathrm{~S}-4 \mathrm{P}_{1 / 2}$ line strength with the laser polarization angle $\theta_{L}$.

Such a behavior is not found in the experimental data as can be seen from Fig. B.2. Shown are the ratios of observed line strengths at laser polarization angles $\theta_{L}$ and $\theta_{L}+90^{\circ}$ for different settings. A small offset of $0.7(2) \%$ from the expected value of one can be traced back to the fact that the two intensities before the fiber have not exactly been stabilized to the small absolute power. Sine fits of the extracted line strength ratios results in a small modulation amplitudes of $0.6(4) \%$ and $0.1(3) \%$. These values are more than one hundred times smaller than the amplitude modulation observed for the $2 \mathrm{~S}-4 \mathrm{P}_{3 / 2}$ transition and compatible with zero. Assuming a 
detection probability that is $0.6 \%$ smaller for the $\pi$ component than for the sigma components, corresponding additional QI line shifts are more than one hundred times smaller than the ones discussed above and therefore negligible on the current level of accuracy.

\section{B.3. Atomic beam parameters and saturation}

All systematic effects which have been discussed in this section are related to the fact that emitted radiation is not detected with equal probability for an arbitrary emission direction $\hat{r}\left(\theta_{d}, \phi_{d}\right)$. In addition to these purely geometrical effects, parameters of the atomic beam as well as the effects of saturation have to be included in the simulation.

Atomic beam parameters that mainly influence the observed QI line shifts are the velocity distribution of $2 \mathrm{~S}$ atoms and the divergence of the atomic beam. As discussed in Sec.6.2, we use a Monte Carlo simulation to model these parameters. The corresponding modification of the predicted QI line shifts amounts to about $6.2 \mathrm{kHz}$ or $17 \%$ of the full model prediction(Fig. 6.3). From a comparison of both, the simulated and experimental line shifts as well as the line widths, the corrections due to atomic beam parameters are estimated to be accurate at a level of at least $20 \%$, i.e. $4 \%$ of the total QI line shift.

Corrections due to saturation and power broadening contribute $9.5 \mathrm{kHz}$, i.e. about $26 \%$. The correction is accurate to at least $20 \%(1.9 \mathrm{kHz})$, i.e. $6 \%$ of the full amplitude. 


\section{Active fiber-based retroreflector}

\section{C.1. Minimization of imperfections}

1 This section discusses additional requirements that have to be fulfilled in order to minimize residual uncompensated Doppler shifts using the active fiber-based retroreflector (AFR). The first one is the use of a low imaging aberration collimator for good beam homogeneity and low phase distortions. Unfortunately these conditions mostly evade a simple quantitative analysis. However, one can get a qualitative picture by observing the transverse intensity profile of the collimated beam at various distances, as shown in Fig. C.1 and Fig. C.3. In principle, aspheric lenses can provide suitably small residual spherical aberration and minimize the number of reflecting surfaces in the beam path. Our experience, however, shows, that this type of lenses is not necessarily a good choice depending on what kind of application they are optimized for and on the surface quality. Due to deviations form the ideal aspheric shape, the produced beam profiles may be not exactly Gaussian as shown in Fig. C.1.

In addition, the retracing of the wave fronts is influenced by the position of the beam waist, which in turn depends on the distance between the fiber tip and the rear principal plane of the collimating objective $d_{f c}$ (see Fig. 3.6). Imaging of the Gaussian beam emerging from PM fiber 1 through the collimating objective to the mirror and back can be calculated using the standard matrix formalism for Gaussian beam propagation in optical systems [69]. The re-coupling losses $L_{r c}$ for a certain distance $d_{f c}$ and distance to the mirror $d_{m}$ are given by the overlap integral of the focused returning beam with waist $w_{r}$ and the fiber mode with waist $w_{0}$. Neglecting wave front curvature, $L_{r c}$ can be approximated by:

$$
L_{r c} \approx \frac{\left(w_{0}-w_{r}\right)^{2}}{\left(w_{0}^{2}+w_{r}^{2}\right)^{2}},
$$

where $w_{r}$ is given by:

$$
\begin{aligned}
w_{r}=\frac{w_{0}}{f^{2}}[ & {\left[2 d_{f c} d_{m}-2\left(d_{f c}+d_{m}\right) f+f^{2}\right]^{2} } \\
& \left.+\left[2\left(d_{f_{c}}-f\right)\left(d_{f c} d_{m}-\left(d_{f c}+d_{m}\right) f\right) \frac{\lambda}{\pi w_{0}^{2}}\right]^{2}\right]^{1 / 2} .
\end{aligned}
$$

For the collimator used in the experiment $(f=27 \mathrm{~mm})$, an axial displacement of only $15 \mu \mathrm{m}$ from the optimum position in $d_{f c}$ results in a re-coupling loss of already

\footnotetext{
${ }^{1}$ The content of this chapter has been published in [25] and was only slightly modified to fit the general structure of this thesis.
} 

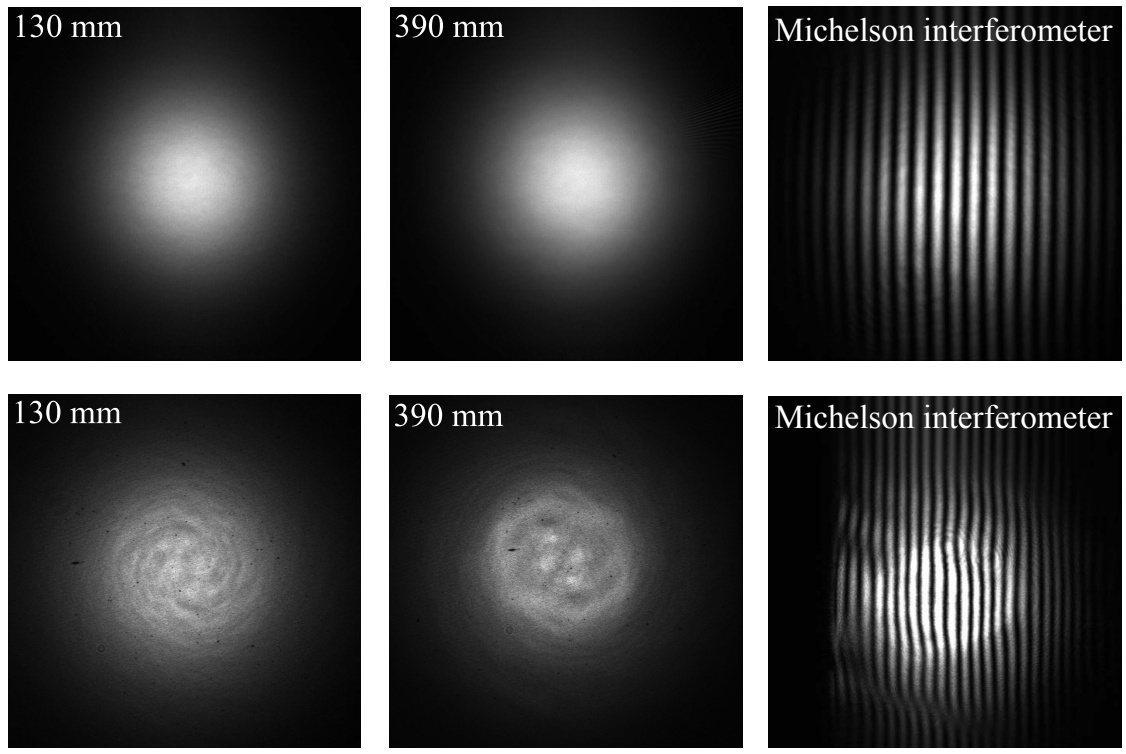

Figure C.1.: Comparison of measured beam profiles for two different collimators tested for the active fiber based retroreflector (AFR). Upper row: collimator used in the current design (two achromatic lens doublets [59], $f=27 \mathrm{~mm}$ ). Bottom row: single aspheric lens $(f=30 \mathrm{~mm})$. Distances correspond to the intersection points of the forward $(130 \mathrm{~mm})$ and the backward $(390 \mathrm{~mm})$ traveling light with the atomic beam (see Fig. 3.6). Due to deviations from the ideal aspheric shape, the beam profiles produced by the aspheric lens are not exactly Gaussian and the wave fronts do not exactly retrace each other. A slightly misaligned Michelson interferometer gives a qualitative estimate on the phase front distortions (right column).

$12 \%$. Remote control of the distance $d_{f c}$ is essential because the refractive index changes when the vacuum chamber housing the experiment is evacuated. If $d_{f c}$ has been optimized in air and is not adjusted after evacuation, we measure a relative transmission loss of $46 \%$ at PD1. A standard translation mount has been equipped with a remote-controlled piezo-actuator-driven micrometer screw to perform this task [70]. The axial position of the collimator $d_{f c}$ is stable enough so that no active feedback is needed and occasional adjustments are sufficient.

The influence of displacements of the collimator from the optimum position in $d_{f c}$ has been estimated based on a numerical simulation solving the optical Bloch equations for the hydrogen $2 \mathrm{~S}-4 \mathrm{P}$ excitation discussed in the main body of this text and is shown in Fig. C.2. We rewrite the Doppler shift in Eq. (3.2) using the parameter $\eta(\delta \alpha)$ that measures the slope of the Doppler shift at an angle $\alpha=90^{\circ} \pm \delta \alpha$ as a function of atom velocity $v$ in $\mathrm{Hz} /(\mathrm{m} / \mathrm{s})$ or simply $\mathrm{m}^{-1}$ :

$$
\Delta \nu_{D}=\eta(\delta \alpha) \times v
$$




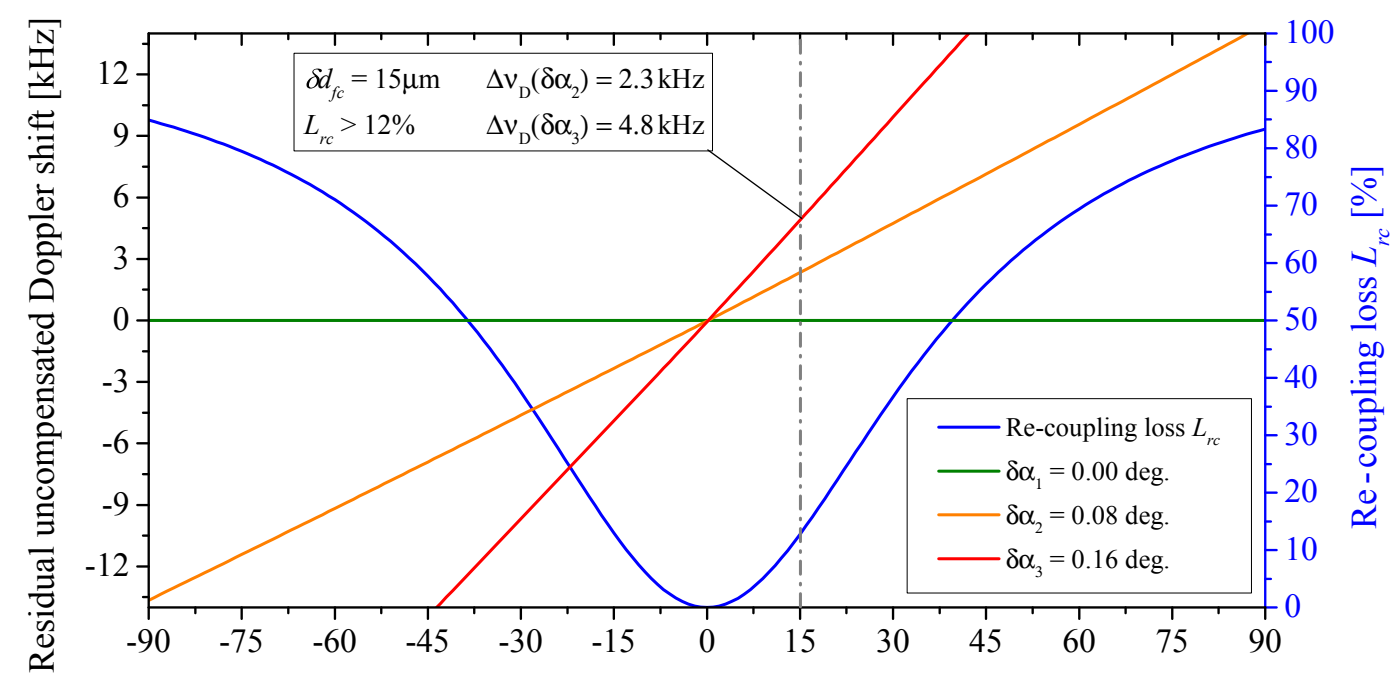

Lens displacement $\delta d_{f c}$ from optimum position $[\mu \mathrm{m}]$

Figure C.2.: Simulation of re-coupling losses (blue, right scale) and residual uncompensated Doppler shift (left scale) for an atom with velocity $v=300 \mathrm{~m} / \mathrm{s}$ as a function of the axial lens displacement $\delta d_{f c}$ for an otherwise ideal active fiber-based retroreflector. The waist size of $2.1 \mathrm{~mm}$ in the simulation corresponds to the one determined for the lens assembly used in the current design (two achromatic lens doublets [59], $f=27 \mathrm{~mm}$ ). The Gaussian beams in forward and backward direction perfectly re-trace each other at $\delta d_{f c}=0$, where the beam waist is located on the surface of the flat high reflecting mirror (Fig. 3.6). Atomic trajectories intersect the laser beams at angles $\delta \alpha_{1}=0 \mathrm{deg}$. (green), $\delta \alpha_{2}=0.08 \mathrm{deg}$. (orange) and $\delta \alpha_{3}=0.16 \mathrm{deg}$. (red). If $\delta d_{f c}=0$, Doppler shifts are perfectly canceled, independent of the laser-to-atomic-beam angle $\alpha=\pi / 2+\delta \alpha$. A small displacement of $\delta d_{f c}=15 \mu \mathrm{m}$ (gray dashed line) results in a re-coupling loss of $12 \%$ already, constituting a sensitive experimental handle on axial collimator misalignment.

where

$$
\eta(\delta \alpha)=\frac{1}{\lambda} \times \sin (\delta \alpha)
$$

Any first order Doppler shift is proportional to the atom velocity (Eq. (C.3)). The proportionality factor $\eta$ can be expressed by Eq. (C.4) for plane waves or locally for any kind of wave front curvature. The definition in Eq. (C.3) is used in Sec.4, where the slope $\eta$ is studied experimentally. The residual uncompensated Doppler shift in the center of weight position extracted from the simulation for the collimator displacement of $15 \mu \mathrm{m}$, i.e. re-coupling loss of $L_{r c}>12 \%$, and an otherwise ideal AFR system is approximately $\eta_{c d}\left(0.08^{\circ}\right) \leq 7.7 \mathrm{~m}^{-1}$. At the thermal mean velocity $\langle v\rangle=$ $300 \mathrm{~m} / \mathrm{s}$ of our sample atoms (cryogenic beam at $5.8 \mathrm{~K}$, see Sec. 3), this corresponds to a residual shift of $2.3 \mathrm{kHz}$ (Fig. C.2). The measured re-coupling efficiency for the optimized system in the experiment, on the other hand, is found to be 100.4 (9) \% as discussed further below. With the high sensitivity to misalignment in $d_{f c}$ brought 
by re-coupling losses, this uncompensated Doppler shift is well under control.

Imbalances in the intensities of the forward and backward traveling beams are largely suppressed by the use of an HR mirror $(R=99.995 \%$ [61]) for retroreflection. The residual uncompensated Doppler shift can be estimated by approximating the resulting line shape by a sum of two Lorentzians with separation $\Delta \nu=2 v \cos (\alpha) / \lambda$ as shown in Fig. 3.5. Assuming small excitation rates, i.e. saturation of the transition is negligible, imbalanced intensities transfer linearly to the respective amplitudes. For small deviations $\delta \alpha$ and $R \in[90 \% ; 100 \%]$, the uncompensated Doppler shift in the center of weight position can be approximated as a linear function of the intensity imbalance $\xi=1-R$ if the separation of the two components is less than their line width:

$$
\Delta \nu_{D, m} \approx \frac{v}{2 \lambda} \times \delta \alpha \times \xi
$$

Note that in contrast to Eq.(3.3), the expression in Eq.(C.5) is linear in $\delta \alpha$, i.e. the corresponding uncompensated Doppler shift is no longer independent of the angle between the atomic and the laser beams. For our spectroscopy of the 2S$4 \mathrm{P}$ transition in atomic hydrogen, the estimate for the uncompensated shift due to intensity imbalance caused by the mirror reflectivity $\left(\xi_{m}=5 \times 10^{-5}\right)$ amounts to only $\eta_{m}<0.23 \mathrm{~m}^{-1}$ even for a large angle $\delta \alpha=0.5^{\circ}$. In contrast to that, this effect is more than three orders of magnitude larger in the case of the aforementioned hollow corner cube retroreflector [51] with a specified $\xi_{c c}$ of $1.2 \times 10^{-1}$ and represents a strong limitation for using such a device directly in the spectroscopy beam path.

Residual imaging aberrations through the collimation optics, in particular spherical aberration and clipping, can cause aberrations in the wave fronts of the Gaussian beams. These deviations deform upon propagation and do not necessarily retrace in the returning beam as can be seen in the bottom row of Fig. C.1 and middle row of Fig. C.3. The lens system of the AFR presented here consists of a combination of two cemented achromatic lens doublets [59]. Although chromatic aberrations are irrelevant due to the use of a narrow-band laser, each individual lens doublet features a reasonably good compensation of the spherical aberration at the same time. Suitable combinations of doublets with low residual spheric aberration have been identified using the free software package WinLens Basic [71] and characterized using standard beam profiling techniques.

A slightly misaligned Michelson interferometer has been used to image wave front differences of the forward and backward traveling beams (Fig. C.1). For the lens system presented in this text, no residual spherical aberration could be detected utilizing either technique.

We illustrate the importance of residual spherical aberration in Fig. C.4 using a numerical simulation solving the optical Bloch equations for the hydrogen 2S$4 \mathrm{P}$ excitation. The Doppler shift is exactly canceled for the ideal case of phaseretracing Gaussian beams. A small angular misalignment $\epsilon$ between the forward and backward traveling beams results in a constant frequency offset according to Eq. (3.3). In the case shown, $\epsilon$ equals $10 \mu \mathrm{rad}$, a limit which can be beaten by our 
Collimator used in AFR $(f=27 \mathrm{~mm})$
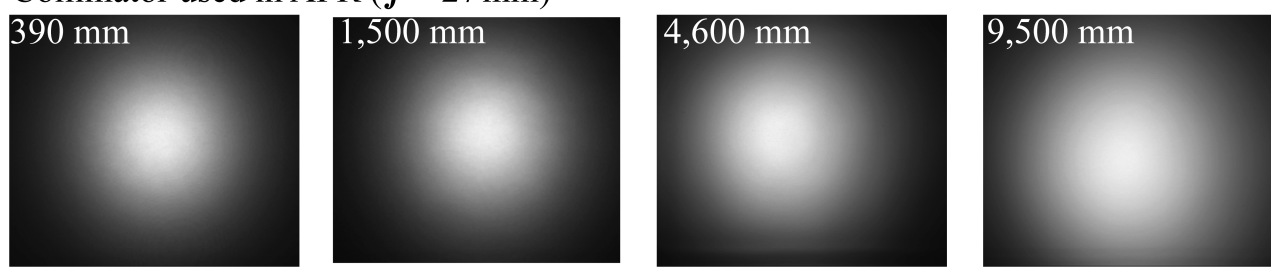

Collimator with spherical aberration $(f=15 \mathrm{~mm})$
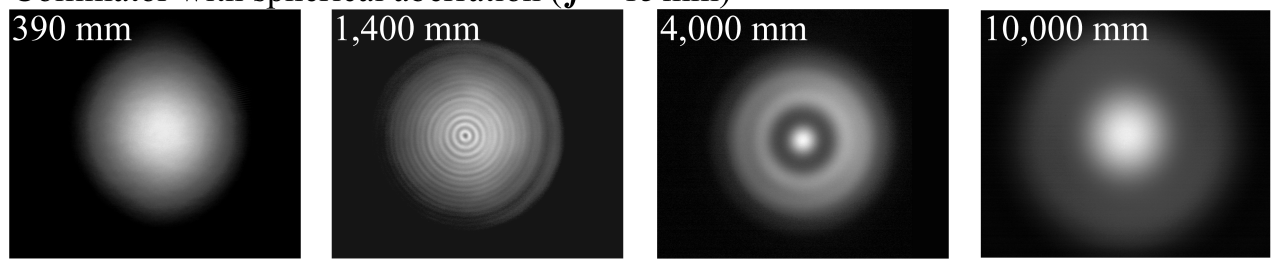

Simulated
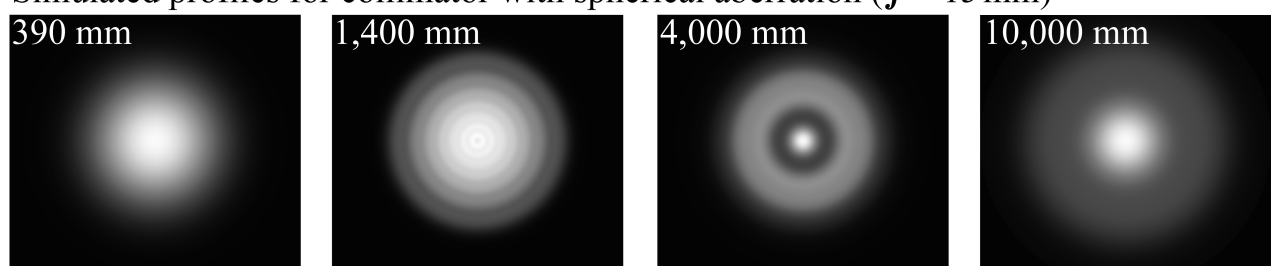

Figure C.3.: Beam profiles acquired for the two collimators discussed in Fig. 3.7c (identical scale in all pictures). Both collimators produce acceptable beam profiles at moderate distances of up to $390 \mathrm{~mm}$. The lens assembly used in the final design ( $f=27 \mathrm{~mm}[59])$ produces a smooth Gaussian intensity profile even at a distance of $9.5 \mathrm{~m}$ (top row). In contrast to that, residual spherical aberrations cause significant radial intensity modulations at much smaller distances in case of the lens assembly used in a legacy version of our AFR ( $f=15 \mathrm{~mm}$, middle row). The simulated beam profiles in the bottom row have been used for estimations of the residual uncompensated Doppler shift due to spherical aberrations in Fig. C.4.

AFR as demonstrated in Sec. 4. Red triangles in Fig. C.4 indicate the line centers obtained for the simulation data including spherical aberration as shown in the bottom row of Fig. C.3.

Using "off-the-shelf" components represents a fast and cost-effective way to proceed. However, it turns out that the quality of the obtained beam profiles critically depends on the specific lenses used, even if they stem from the same production batch. Speckle patterns may be present, most probably caused by differences in the cementing process of the achromats. Therefore, we plan to use a multiplet of airspaced spheric lenses for the next generation of the experiment which is intended to probe the 2S-6P transition at $410 \mathrm{~nm}$ [72]. A total number of three individual lenses including one with negative focal distance is sufficient to compensate for spherical aberrations.

Another qualitative estimation for the proper implementation of the AFR can be 


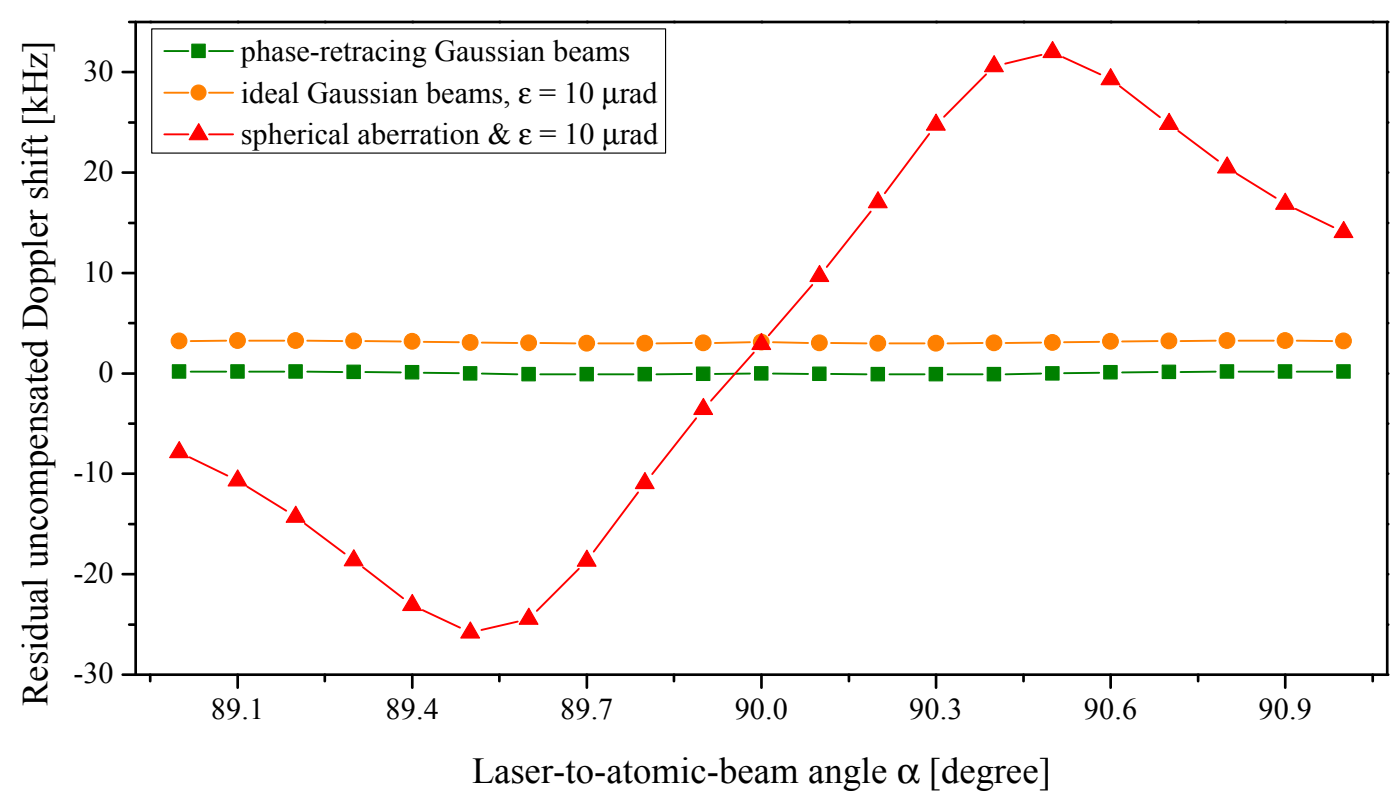

Figure C.4.: Uncompensated residual Doppler shift for an atom traveling at $v=300 \mathrm{~m} / \mathrm{s}$ and different imperfections of the active fiber-based retroreflector (AFR). For an ideal AFR, the Doppler shift is compensated independent of the laser-to-atomic-beam angle $\alpha$ (green squares). The case $\overrightarrow{k_{1}}+\overrightarrow{k_{2}} \neq 0$ ( $\varepsilon=10 \mu \mathrm{rad}$ for the case shown) for an otherwise ideal AFR leads to a constant shift of the observed resonance, where the offset is defined by Eq. 3.3 (orange circles). The presence of spherical aberrations (see simulated beam profiles in Fig. C.3) leads to $\alpha$-dependent shifts of several tens of kilohertz (red triangles).

obtained by measuring the amount of power that is coupled back into PM fiber 1 . The re-coupling efficiency of an ideal AFR is unity (see Fig. C.2). The re-coupling efficiency as defined by the overlap integral of the returning beam with the fiber mode cannot directly be measured in the experimental implementation. Instead, the power returning back through the fiber has to be measured after the beam splitter BS in Fig. 3.6. Losses due to different elements have to be considered in order to calculate the amount of power that has actually been coupled back into the fiber. In particular, these elements are the fiber end facets $(R \leq 0.1 \%)$, the collimation objective of the $\operatorname{AFR~}(R \leq 0.1 \%)$, the absorption of PM fiber $1(A \leq 0.15 \mathrm{~dB})$, the collimation lens CL on the other end of PM fiber $1(R \leq 0.5 \%)$, one additional mirror $(R=96.3(5) \%$, not shown in Fig. 3.6) and the 50/50 beam splitter BS used to separate a fraction of the returning light $(T=50.3(5) \%)$. Using these values, we infer a re-coupling efficiency of 100.4 (9) \% back into the fiber. This value should of course be smaller than $100 \%$ and we give a $90 \%$ confidence level lower limit of $99.0 \%$ re-coupling efficiency. At the given level of measurement accuracy, the re-coupling efficiency is indistinguishable from the $100 \%$ limit expected from an ideal AFR.

Light that is not mode matched with the fiber mode upon re-coupling, either due to a displacement of the collimator or residual imaging aberrations, is mostly 
scattered off the fiber ferrule. Since the fiber tip is cleaved at an angle of $8^{\circ}$ and the distance to the spectroscopy region is sufficiently long $(130 \mathrm{~mm})$, the influence of the scattered light on the spectroscopy signal is expected to be small. In addition, proper anti-reflection coatings on all relevant optics, especially the collimator lenses [59] and the fiber end facets [58], suppress parasitic Fabry Pérot etalons. We tested for the latter by measuring the transmission of the HR mirror (power monitor, Fig. 3.6) and found no significant frequency dependence of the light intensity.

While certain imperfections listed in this section are hard to model, we can directly measure the corresponding residual uncompensated Doppler shifts in our spectroscopy experiment discussed in Sec. 4.

A PANDA type polarization maintaining (PM) fiber is used to provide a well defined polarization in our experiment. The fiber is equipped with stress rods in the cladding material that define the preferred PM fiber axes. The induced stress may also deform the fiber core, leading to sightly different beam diameters and shapes depending the input polarization. However, we do not see any significant influence of the input polarization on the residual Doppler shift and thus average data independent of the input polarization in Sec. 4.

\section{C.2. Active Stabilization}

We use a dither method to stabilize the re-coupling into the fiber to its maximum value, i.e. maximize the retracing of the forward and backward traveling waves. The mount holding the HR mirror is equipped with two piezo actuator driven micrometer screws for coarse adjustment and two piezo actuators (PZTs) for fine adjustment and stabilization [73]. The PZT voltages are modulated by sine waves at frequencies of $1100 \mathrm{~Hz}$ and $876 \mathrm{~Hz}$ for tip and tilt, respectively, where we find acceptably small cross-talk between the two channels. Alternatively, a single modulation frequency and a $90^{\circ}$ phase shift combined with with a two-channel lock-in amplifier may be used [54].

The mirror tip and tilt angles were calibrated as a function of the PZT voltage by measuring the laser beam deflection for different voltages at a distance of about $5 \mathrm{~m}$. In the linear regime of the PZT (below $50 \mathrm{~V}$ ) the coefficient is found to be $9.9 \mathrm{\mu rad} / \mathrm{V}$ in both directions and used to infer the angular beam misalignment in Fig. 3.7c. The modulation amplitudes can be determined to be $1.0 \mu \mathrm{rad}$ and $0.4 \mathrm{urad}$, respectively, from the measured intensity modulation at PD1 and using Fig. 3.7c. These amplitudes correspond to a small Doppler shift of maximally $0.3 \mathrm{kHz}$ at $v=300 \mathrm{~m} / \mathrm{s}$, which is averaged to zero in our experiment due to the fact that the integration time of about 1 second in the data acquisition (Sec. 3.2) is much longer than the time scale of the modulation. The error signals for the PI-controllers in each channel (tip and tilt) are obtained by demodulation of the photo diode signal (PD1 in Fig. 3.6) using lock-in amplifiers (2× Stanford Research Systems SR530). Since it is critical to maintain zero offset of the locking loop, a calibration of the 


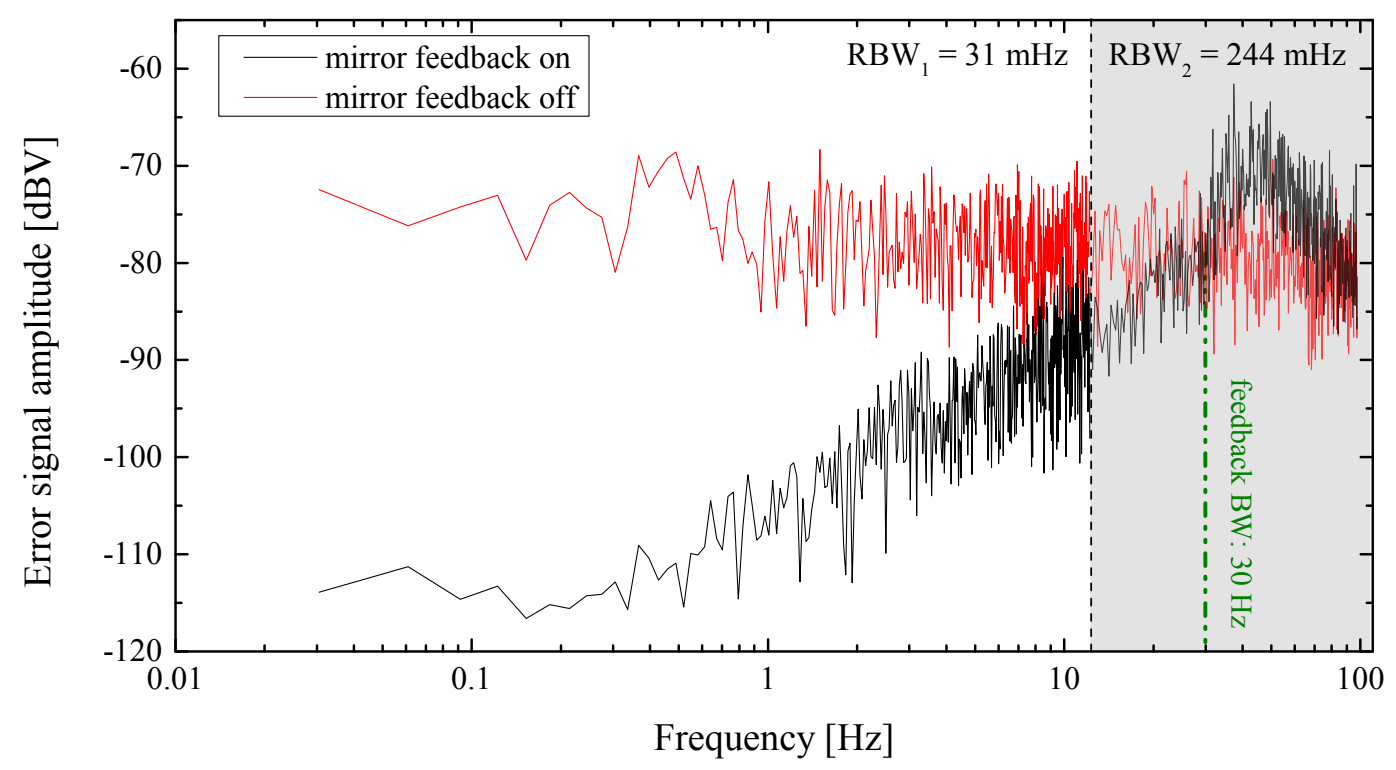

Figure C.5.: FFT trace of the in-loop error signal produced by the lock-in amplifier in one of the AFR mirror feedback channels (tilt). The frequency interval $[31 \mathrm{mHz} ; 12.2 \mathrm{~Hz}]$ has been recorded with a resolution band width $\left(\mathrm{RBW}_{1}\right)$ of $31 \mathrm{mHz}$ (left of black dashed line), for the interval $[12.2 \mathrm{~Hz} ; 97.4 \mathrm{~Hz}] \mathrm{RBW}_{2}=244 \mathrm{mHz}$ (right of black dashed line). The difference in RBW has been accounted for by scaling the amplitudes measured in the second interval by the ratio $\mathrm{RBW}_{1} / \mathrm{RBW}_{2}$. The feedback bandwidth of $30 \mathrm{~Hz}$ (green dashed line) is determined by the crossing point of the noise figures in the stabilized (black) and unstabilized case (red). A servo bump is located at about $45 \mathrm{~Hz}$.

lock-in amplifier and the PI-controller offsets should be performed. The input of the lock-in amplifier must be short-circuited or 50 Ohm terminated for this purpose while the reference frequency and settings are all maintained. The lock-in output is then fed into the error-input channel of the controller. Finally, with the P(I)controller engaged (integrator off), the controller input offset is adjusted such that its output becomes zero. The AFR mirror feedback is optimized and characterized via the in-loop error signals after demodulation by the lock-in amplifiers. Fig. C.5 shows the FFT spectrum of the in-loop error signal in one of the channels (tilt) in the range between $31 \mathrm{mHz}$ and $98 \mathrm{~Hz}$. The feedback bandwidth is found to be about $30 \mathrm{~Hz}$, indicated by the green dashed-dotted line at the crossing point of the noise figures in the stabilized and unstabilized case. The feedback servo bump is found at a frequency of $45 \mathrm{~Hz}$. Measurements of the spectral noise figures in the second channel (tip) shows very similar results. 


\section{Theory of quantum interference from neighboring atomic transitions}

\section{D.1. Dipole matrix elements for hydrogen}

Eqs. (2.12) - (2.14) used in the perturbative approach of calculating the 2S-4P lines shapes for our investigation of QI line shifts and Eq. (2.18) in the master-equation approach involve a number of dipole matrix elements which need to be calculated in order to set up the respective equations.

The Wigner-Eckart-Theorem ([37] Eq. (4.120)) allows to decompose the dipole matrix elements into a factor that contains the geometry (i.e. $z$-components of the involved angular momenta) in form of a $3 \mathrm{j}$ symbol, and a the reduced matrix element that is independent of it:

$$
\begin{aligned}
& \left\langle n(I J) F M_{F}\left|D^{\nu}\right| n^{\prime}\left(I J^{\prime}\right) F^{\prime} M_{F}^{\prime}\right\rangle= \\
& (-1)^{F-M_{F}}\left(\begin{array}{ccc}
F & 1 & F^{\prime} \\
-M_{F} & \nu & M_{F}^{\prime}
\end{array}\right)\left\langle n,(I J) F\|D\| n^{\prime},\left(I J^{\prime}\right) F^{\prime}\right\rangle
\end{aligned}
$$

It is assumed in this expression that the nuclear angular momentum $I$ and the electronic angular momentum $J$ are coupled to the total angular momentum of the atom $F$ with $z$-component $M_{F}$. The principle quantum numbers of the states are given by $n$ and $n^{\prime}$. The nuclear angular momentum is associated only with a magnetic dipole moment but not with an electric dipole moment. Therefore the dipole operator does not act on $I$. In this situation the reduced matrix elements can be further reduced ([37] Eq. (4.175)):

$$
\begin{aligned}
& \left\langle n(I J) F\|D\|\left(I J^{\prime}\right) F^{\prime}\right\rangle= \\
& (-1)^{I+J+F^{\prime}+1} \sqrt{(2 F+1)\left(2 F^{\prime}+1\right)} \times \\
& \times\left\{\begin{array}{ccc}
J & F & I \\
F^{\prime} & J^{\prime} & 1
\end{array}\right\}\left\langle n, J\|D\| n^{\prime}, J^{\prime}\right\rangle
\end{aligned}
$$

Since the electronic angular momentum is in turn composed of two angular momenta we have $\left\langle n, J\|D\| n^{\prime}, J^{\prime}\right\rangle=\left\langle n,(S L) J\|D\| n^{\prime},\left(S L^{\prime}\right) J^{\prime}\right\rangle$ of which the electron spin is not associated with an electric dipole moment. The reduced matrix elements can 
therefore be further reduced by extracting another $6 \mathrm{j}$ symbol:

$$
\begin{aligned}
& \left\langle n(S L) J\|D\| n^{\prime}\left(S L^{\prime}\right) J^{\prime}\right\rangle= \\
& (-1)^{S+L+J^{\prime}+1} \sqrt{(2 J+1)\left(2 J^{\prime}+1\right)} \times \\
& \times\left\{\begin{array}{ccc}
L & J & S \\
J^{\prime} & L^{\prime} & 1
\end{array}\right\}\left\langle n, L\|D\| n^{\prime}, L^{\prime}\right\rangle
\end{aligned}
$$

The last reduced matrix element $\left\langle n, L\|D\| n^{\prime}, L^{\prime}\right\rangle$ is given for hydrogen in analytical from in [74] Eqs. (63.2) and (63.5). Putting together Eqs. (D.2) - (D.4), the dipole matix elements used in Eqs. (2.12) - (2.14) and Eq. (2.18) can be written as:

$$
\begin{aligned}
D_{e, f}^{\nu} & = \\
& (-1)^{F_{f}-M_{F_{f}}}\left(\begin{array}{ccc}
F_{f} & 1 & F_{e} \\
-M_{F_{f}} & \nu & M_{F_{e}}
\end{array}\right)\left\langle n_{f}, L_{f}\|D\| n_{e}, L_{e}\right\rangle \\
& \times(-1)^{J_{f}+I+F_{e}+1} \sqrt{\left(2 F_{f}+1\right)\left(2 F_{e}+1\right)}\left\{\begin{array}{ccc}
J_{f} & F_{f} & I \\
F_{e} & J_{e} & 1
\end{array}\right\} \\
& \times(-1)^{L_{f}+S+J_{e}+1} \sqrt{\left(2 J_{f}+1\right)\left(2 J_{e}+1\right)}\left\{\begin{array}{ccc}
L_{f} & J_{f} & S \\
J_{e} & L_{e} & 1
\end{array}\right\}
\end{aligned}
$$

where $n_{f}, F_{f}, M_{F_{f}}, J_{f}$, and $L_{f}$ are the quantum numbers of the final state, $n_{e}, F_{e}$, $M_{F_{e}}, J_{e}$, and $L_{e}$ are the quantum numbers of the excited state, and $I$ and $S$ are the nuclear and electronic spin, that are the same for all states. An analogous expression for the absorbing dipole moment is obtained by replacing $e \rightarrow i, f \rightarrow e$ and $\nu \rightarrow \eta$. We set the reduced matrix element to 1 as it is a common factor to all Zeeman, fineand hyperfine components. The $3 \mathrm{j}$ and $6 \mathrm{j}$ symbols may be computed with the Racah formula $[75,76]$ or Mathematica ${ }^{1}$. As can be seen from the Racah formula, both the $3 \mathrm{j}$ and $6 \mathrm{j}$ are given as square roots of (mostly small) fractions. Special cases, that should cover all practically cases are given in [37] Eqs. (4.2.2) and (4.24).

\section{D.2. Arbitrary laser polarization}

As mentioned in Sec. 2.2.2 before, the intensity distribution of the scattered light for linear laser polarization along the $z$-axis of the incident light (or circular polarization about the $z$-axis) can be calculated with $\eta=0$ and $\eta= \pm 1$, respectively, using Eq. (2.12) or Eq. (2.13). Any other polarization vector $\overrightarrow{\epsilon_{L}}$ has to be expressed in terms of its spherical components and the dipole moment $D_{i, e}^{\eta}$ in Eq. (2.9) is replaced by the scalar product:

$$
\vec{D}_{i, e} \cdot \overrightarrow{\epsilon_{L}}=\sum_{\eta}\left[D_{i, e}^{\eta}\right]^{*} \epsilon_{\eta}
$$

\footnotetext{
${ }^{1}$ For $6 \mathrm{j}$ symbols that do not satisfy one of the triangle conditions, Mathematica may issue an error massage instead of setting them to zero. This problem can be circumvented by re-defining this function.
} 
In that case the Eq. (D.14) has to be used instead of Eq. (D.15) as will be discussed in Sec. D.3. Linear laser polarization in the direction $\left(\theta_{L}, \varphi_{L}\right)$ for example yields for $\epsilon_{p^{\prime}}$ :

$$
\begin{aligned}
\epsilon_{-1} & =+\frac{1}{\sqrt{2}}\left(\sin \left(\theta_{L}\right) \cos \left(\varphi_{L}\right)-i \sin \left(\theta_{L}\right) \sin \left(\varphi_{L}\right)\right) \\
\epsilon_{0} & =\cos \left(\theta_{L}\right) \\
\epsilon_{+1} & =-\frac{1}{\sqrt{2}}\left(\sin \left(\theta_{L}\right) \cos \left(\varphi_{L}\right)+i \sin \left(\theta_{L}\right) \sin \left(\varphi_{L}\right)\right)
\end{aligned}
$$

\section{D.3. Emitted Intensity Pattern}

The far field of a dipole $\overrightarrow{\mathcal{D}}$ oscillating with frequency $\omega_{0}$ and located at the origin can be described with classical electrodynamics (see also Sec. 2.1):

$$
\vec{E}(\vec{r}) \propto(\vec{r} \times \overrightarrow{\mathcal{D}}) \times \vec{r} \frac{e^{i \omega_{0} t-i k r}}{r^{3}}
$$

Here $\vec{r}$ is the detection point and $r=|\vec{r}|$ the distance from the origin. For the analysis of the quantum interference line shape distortions, only the direction of the emitted field matters and the last factor in Eq. (D.9) is ignored. This direction is described by $(\theta, \varphi)$ in spherical coordinates ${ }^{2}$ (see Fig. 2.3) whose unit vectors are:

$$
\vec{e}_{r}=\left(\begin{array}{c}
\sin (\theta) \cos (\varphi) \\
\sin (\theta) \sin (\varphi) \\
\cos (\theta)
\end{array}\right), \quad \vec{e}_{\theta}=\left(\begin{array}{c}
\cos (\theta) \cos (\varphi) \\
\cos (\theta) \sin (\varphi) \\
-\sin (\theta)
\end{array}\right), \quad \vec{e}_{\varphi}=\left(\begin{array}{c}
-\sin (\varphi) \\
\cos (\varphi) \\
0
\end{array}\right)
$$

Plugging the spherical components of $\overrightarrow{\mathcal{D}}$

$$
\overrightarrow{\mathcal{D}}=\left(\begin{array}{c}
\frac{1}{\sqrt{2}}\left(\mathcal{D}_{-1}-\mathcal{D}_{+1}\right) \\
\frac{i}{\sqrt{2}}\left(\mathcal{D}_{-1}+\mathcal{D}_{+1}\right) \\
\mathcal{D}_{0}
\end{array}\right)
$$

into $(\vec{r} \times \overrightarrow{\mathcal{D}}) \times \vec{r}$, an expression for the emitted field $\vec{E}(\vec{r})$ for each spherical component $q$ is obtained that might be used to verify that the field is transversal, i.e. $\overrightarrow{e_{r}} \cdot \vec{E}(\vec{r})=0$. The two polarization components along $\vec{e}_{\theta}$ and $\vec{e}_{\varphi}$ are given by:

$$
\begin{aligned}
E_{\theta}(\theta, \varphi) & \equiv \vec{e}_{\theta} \cdot \vec{E}(\vec{r}) \\
& \propto-\sin (\theta) \mathcal{D}_{0}+\frac{\cos (\theta)}{\sqrt{2}}\left[\left(\mathcal{D}_{-1}-\mathcal{D}_{+1}\right) \cos (\varphi)+i\left(\mathcal{D}_{-1}+\mathcal{D}_{+1}\right) \sin (\varphi)\right] \\
E_{\varphi}(\theta, \varphi) & \equiv \vec{e}_{\varphi} \cdot \vec{E}(\vec{r}) \\
& \propto-\frac{\sin (\varphi)}{\sqrt{2}}\left(\mathcal{D}_{-1}-D_{+1}\right)+i \frac{\cos (\varphi)}{\sqrt{2}}\left(\mathcal{D}_{-1}+\mathcal{D}_{+1}\right)
\end{aligned}
$$

\footnotetext{
${ }^{2}$ Not to be confused with the spherical components introduced before.
} 
In the semi-classical ansatz, used both in the perturbative approach as well as in the master-equation approach, the excitation and fluorescence light is treated classically and the classical dipole emission patterns can be adapted. Therefore the sum of the scattering matrix elements $\sum_{e} S_{e, i \rightarrow f}^{\nu, \eta}$ in Eq. (2.9) are identified with the classical dipole moments $\mathcal{D}_{\nu}$.

The possibility of several final states being involved is taken into account by adding the respective intensities incoherently. As discussed before (Sec. 2) this justified because only pathways that lead from a common initial to a common final state interfere. The summation over final states yields:

$$
\begin{aligned}
I_{\eta}(\theta, \varphi) & \propto\left|\vec{e}_{\theta} \cdot \vec{E}(\vec{r})\right|^{2}+\left|\vec{e}_{\varphi} \cdot \vec{E}(\vec{r})\right|^{2} \\
& \propto \sum_{f}\left|\sum_{e}\left(\sin (\theta) S_{e, i \rightarrow f}^{0, \eta}+\frac{\cos (\theta)}{\sqrt{2}}\left(S_{e, i \rightarrow f}^{+1, \eta} e^{-i \varphi}-S_{e, i \rightarrow f}^{-1, \eta} e^{+i \varphi}\right)\right)\right|^{2} \\
& +\sum_{f}\left|\sum_{e}\left(\frac{e^{-i \varphi}}{\sqrt{2}} S_{e, i \rightarrow f}^{+1, \eta}+\frac{e^{+i \varphi}}{\sqrt{2}} S_{e, i \rightarrow f}^{-1, \eta}\right)\right|^{2}
\end{aligned}
$$

with $S_{e, i \rightarrow f}^{\nu, \eta}$ given in Eq. (2.9) and the matrix elements in Eq. (D.5). The dependence of $I_{\eta}(\theta, \varphi)$ on the initial state (i) and the laser polarization is not written explicitly for brevity. The sums over the excited states should extend over all levels $J_{e}, F_{e}, M_{F_{e}}$ that are close to resonant in the sense of the rule of thumb given in Sec. 2. The sum over the final states should extend over all levels $J_{f}, F_{f}, M_{F_{f}}$ to which transitions are detected. The intensities of the spherical components may also be added up incoherently for laser polarization that can be described by a single spherical component $\eta$ :

$$
\begin{aligned}
I_{\eta}(\theta, \varphi) & \propto\left|\overrightarrow{e_{\theta}} \cdot \vec{E}(\vec{r})\right|^{2}+\left|\vec{e}_{\varphi} \cdot \vec{E}(\vec{r})\right|^{2} \\
= & \sum_{f}\left[\sin ^{2}(\theta)\left(\left|\sum_{e} S_{e, i \rightarrow f}^{0, \eta}\right|^{2}\right)+\frac{1}{2}\left(\cos ^{2}(\theta)+1\right)\left(\left|\sum_{e} S_{e, i \rightarrow f}^{+1, \eta}\right|^{2}+\left|\sum_{e} S_{e, i \rightarrow f}^{-1, \eta}\right|^{2}\right)\right]
\end{aligned}
$$

\section{D.3.1. Interference of spherical components}

The terms appearing in Eqs. (2.12-2.14) have the form $\sum_{e, e^{\prime}} S_{e, i \rightarrow f}^{\nu, \eta}\left(S_{e^{\prime}, i \rightarrow f}^{\nu^{\prime}, \eta}\right)^{*}$ and are proportional to the following $3 \mathrm{j}$ symbols:

$$
\begin{array}{r}
\left(\begin{array}{ccc}
F_{f} & 1 & F_{e} \\
-M_{F_{f}} & \nu & M_{F_{e}}
\end{array}\right) \times\left(\begin{array}{ccc}
F_{e} & 1 & F_{i} \\
-M_{F_{e}} & \eta & M_{i}
\end{array}\right) \\
\times\left(\begin{array}{ccc}
F_{f} & 1 & F_{e}^{\prime} \\
-M_{F_{f}} & \nu^{\prime} & M_{F_{e}}^{\prime}
\end{array}\right) \times\left(\begin{array}{ccc}
F_{e}^{\prime} & 1 & F_{i} \\
-M_{F_{e}}^{\prime} & \eta & M_{i}
\end{array}\right)
\end{array}
$$

Conservation of the $z$-component of the angular momentum requires that each of the $3 \mathrm{j}$ symbols vanish unless the sum of the lower row vanishes. This means that $M_{F_{f}}=\nu+M_{F_{e}} \wedge M_{F_{e}}=\eta+M_{i} \Rightarrow M_{F_{f}}=\nu+\eta+M_{F_{i}}$ and $M_{F_{f}}=\nu^{\prime}+M_{F_{e}}^{\prime} \wedge M_{F_{e}}^{\prime}=$ 
$\eta+M_{i} \Rightarrow M_{F_{f}}=\nu^{\prime}+\eta+M_{F_{i}}$. Subtracting the two resulting equations yields that only terms with $\nu=\nu^{\prime}$ contribute to the sum and hence no mixing between the spherical components occurs such that Eq. (D.15) follows from Eq. (D.14). The physical argument for this behaviour is the following: Interference takes place if and only if pathways lead from a common initial to a common final state. For a given excitation polarization $\eta$ only one emission polarization $\nu$ obeys angular momentum conservation. The $\nu=(-1,0,+1)$ contributions are referred the $\sigma^{-}, \pi$ and $\sigma^{+}$ components. The intensities emitted along the positive $z$-axis $(\theta=0)$ are $(1,0,1)$ for the $\left(\sigma^{-}, \pi, \sigma^{+}\right)$components provided that the $\sum_{f}\left|\sum_{e} S_{e, i \rightarrow f}^{\nu, \eta}\right|^{2}$ are the same for all $\nu$, which is indeed the case (see below). The intensities perpendicular to the $z$-axis $(\theta=\pi / 2)$ are $(1 / 2,1,1 / 2)$ in the same relative units. This means that the $\pi$ component vanishes when observed along the $z$-axis while the $\sigma$ components are only half as strong when observed perpendicular to the $z$-axis. In addition one sees that all components contribute equally if the intensities along the $x, y$ and $z$-axis are added. However, the $\sigma$, and $\pi$ components can only be detected selectively with certain polarizations and/or geometries.

The expression in Eq. (D.15) is the simplest form of the detected intensity and can be used for the description of excitation by a single spherical component $\eta$. The emitted intensity is rotational symmetric around the z-axis for each of the spherical components $\nu$ and hence the $\varphi$ dependence will be dropped. Arbitrary laser polarizations are described by a coherent superposition of spherical components $\eta$ and $\eta^{\prime}$ in which case the angular momentum condition of the previous paragraph has to be replaced by $\nu+\eta=\nu^{\prime}+\eta^{\prime}$ (see Sec. D.2). In that case the Eq. (D.14) has to be used instead of Eq. (D.15) and the the $\varphi$ dependence has to be considered.

\section{D.3.2. Line shape distortions}

To find out about the line shape distortions caused by the quantum interference one may first compute the intensity distribution without them. This is done by adding the contributions from coherently excited quantum paths incoherently:

$$
\begin{aligned}
I_{\eta}^{\mathrm{NQI}}(\theta, \varphi) & \propto \sum_{f, e}\left|\left(\sin (\theta) S_{e, i \rightarrow f}^{0, \eta}+\frac{\cos (\theta)}{\sqrt{2}}\left(S_{e, i \rightarrow f}^{+1, \eta} e^{-i \varphi}-S_{e, i \rightarrow f}^{-1, \eta} e^{+i \varphi}\right)\right)\right|^{2} \\
& +\sum_{f, e}\left|\left(\frac{e^{-i \varphi}}{\sqrt{2}} S_{e, i \rightarrow f}^{+1, \eta}+\frac{e^{+i \varphi}}{\sqrt{2}} S_{e, i \rightarrow f}^{-1, \eta}\right)\right|^{2}
\end{aligned}
$$

An expression analog to Eq. (D.15) may be used if excitation takes place with a laser whose polarization can be described by a single spherical component $\eta$. The line shape distortions due to quantum interference are obtained by the difference between Eq. (D.15) and Eq. (D.17):

$$
I_{\eta}^{\mathrm{QI}}(\theta, \varphi)=I_{\eta}(\theta, \varphi)-I_{\eta}^{\mathrm{NQI}}(\theta, \varphi)
$$




\section{D.3.3. Finite detection angle}

A typical experimental arrangement for the fluorescence detection is a rotationally symmetric detector of detection opening angle $\theta_{c}$ (Fig. 2.3). Then the collected signal is given by the following integral:

$$
I_{\eta}^{\theta_{c}}=\int_{0}^{2 \pi} \int_{0}^{\theta_{c}} I_{\eta}(\theta, \varphi) \sin (\theta) d \theta d \varphi
$$

\section{Detection in $4 \pi$ solid angle}

In the limiting case of an opening angle $\theta_{c}=\pi$, all fluorescence is detected. Using the radiation pattern of the classical dipole given in Eq. (D.13) and Eq. (D.14), the integral can be solved analytically:

$$
\int_{0}^{\pi} \int_{0}^{2 \pi}\left[\left|E_{\theta}(\theta, \varphi)\right|^{2}+\left|E_{\varphi}(\theta, \varphi)\right|^{2}\right] \sin (\theta) d \theta d \varphi=\frac{8 \pi}{3}\left(\mathcal{D}_{-1}^{2}+\mathcal{D}_{0}^{2}+\mathcal{D}_{+1}^{2}\right)
$$

Just like for this gives the full emission of the dipole, as expected when integrating over all directions and the line shape distortions due to quantum interference vanish as discussed before (Sec. 2.4.3).

\section{D.4. Expanded Line Shape}

\section{D.4.1. General treatment}

Analogous to the treatment in the main body of this thesis, we follow the analysis in [33] and separate the sum over the line resonances in Eq. (D.15) into the main perturbed resonance $|e\rangle$ and the non-resonant neighboring (perturbing) resonances $\left|e^{\prime}\right\rangle$. Using Eq. (2.9) we obtain:

$$
\begin{aligned}
\left|\sum_{e} S_{e, i \rightarrow f}^{\nu, \eta}\right|^{2} & =\frac{\left(D_{i, e}^{\eta}\right)^{2}\left(D_{e, f}^{\nu}\right)^{2}}{\omega_{L}^{2}+\left(\Gamma_{e} / 2\right)^{2}} \\
& +\omega_{L} \sum_{e^{\prime} \neq e} \sum_{e^{\prime \prime} \neq e} \frac{D_{i, e^{\prime}}^{\eta} D_{e^{\prime}, f}^{\nu} D_{i, e^{\prime \prime}}^{\eta} D_{e^{\prime \prime}, f}^{\nu}}{\left(\omega_{e^{\prime}}-\omega_{e}\right)^{2}\left(\omega_{e^{\prime \prime}}-\omega_{e}\right)^{2}}\left(\omega_{e^{\prime}}+\omega_{e^{\prime \prime}}-2 \omega_{e}\right) \\
& -2 \omega_{L} \frac{D_{i, e}^{\eta} D_{e, f}^{\nu}}{\omega_{L}^{2}+\left(\Gamma_{e} / 2\right)^{2}} \sum_{e^{\prime} \neq e} \frac{D_{i, e^{\prime}}^{\eta} D_{e^{\prime}, f}^{\nu}}{\omega_{e^{\prime}}-\omega_{e}}
\end{aligned}
$$

Without loss of generality, the resonance frequency $\omega_{e i}$ has been put to zero. Damping terms $\Gamma_{e^{\prime}}$ for the non-resonant terms are neglected. Note that the matrix elements $D^{\nu}$ are real. In comparison with Eq. (9) of [33] we have left out the counter rotating terms $\left(B_{i}\left(\varepsilon_{L}, \varepsilon_{s}\right)\right)$, i.e. neglected the Bloch-Siegert shift, which is at the $\mathrm{mHz}$ level 
for the $2 \mathrm{~S}-4 \mathrm{P}$ transition. The emitted intensity in Eq. (D.15) can now be written as:

$$
\begin{aligned}
I_{\eta}(\theta, \varphi) & \propto \sum_{f}\left[\sin ^{2}(\theta)\left(\left|\sum_{e} S_{e, i \rightarrow f}^{0, \eta}\right|^{2}\right)\right] \\
& +\sum_{f}\left[\frac{1}{2}\left(\cos ^{2}(\theta)+1\right)\left(\left|\sum_{e} S_{e, i \rightarrow f}^{+1, \eta}\right|^{2}+\left|\sum_{e} S_{e, i \rightarrow f}^{-1, \eta}\right|^{2}\right)\right] \\
& \equiv \frac{a_{1}}{\omega_{L}^{2}+\left(\Gamma_{e} / 2\right)^{2}}+a_{2} \omega_{L}+\frac{a_{4} \omega_{L}}{\omega_{L}^{2}+\left(\Gamma_{e} / 2\right)^{2}}
\end{aligned}
$$

with

$$
\begin{gathered}
a_{1}=\left(D_{i, e}^{\eta}\right)^{2} \sum_{f}\left[\frac{1}{2}\left(\cos ^{2}(\theta)+1\right)\left(\left(D_{e, f}^{-1}\right)^{2}+\left(D_{e, f}^{+1}\right)^{2}\right)+\sin ^{2}(\theta)\left(D_{e, f}^{0}\right)^{2}\right] \\
a_{2}=\sum_{f} \sum_{e^{\prime} \neq e} \sum_{e^{\prime \prime} \neq e} \frac{D_{i, e^{\prime}}^{\eta} D_{i, e^{\prime \prime}}^{\eta}\left(\omega_{e^{\prime}}+\omega_{e^{\prime \prime}}-2 \omega_{e}\right)}{\left(\omega_{e^{\prime}}-\omega_{e}\right)^{2}\left(\omega_{e^{\prime \prime}}-\omega_{e}\right)^{2}} \\
\times\left[\frac{1}{2}\left(\cos ^{2}(\theta)+1\right)\left(D_{e^{\prime}, f}^{-1} D_{e^{\prime \prime}, f}^{-1}+D_{e^{\prime}, f}^{+1} D_{e^{\prime \prime}, f}^{+1}\right)\right. \\
\left.\quad+\sin ^{2}(\theta) D_{e^{\prime}, f}^{0} D_{e^{\prime \prime}, f}^{0}\right] \\
a_{4}=-2 \sum_{f} D_{i, e}^{\eta} \sum_{e^{\prime} \neq e} \frac{D_{i, e^{\prime}}^{\eta}}{\omega_{e^{\prime}}-\omega_{e}} \\
\times\left[\frac{1}{2}\left(\cos ^{2}(\theta)+1\right)\left(D_{e, f}^{-1} D_{e^{\prime}, f}^{-1}+D_{e, f}^{+1} D_{e^{\prime}, f}^{+1}\right)\right. \\
\left.+\sin ^{2}(\theta) D_{e, f}^{0} D_{e^{\prime}, f}^{0}\right] .
\end{gathered}
$$

The constant term $a_{3}$ has been dropped since it is usually absorbed in the fit of a flat background. Although the sums look rather lengthy in the general case, they actually do not contain a large of terms in most cases. In particular, if there is only one perturbing transition like in the case of hydrogen 2S-4P (Fig. 2.4), the sums over $e^{\prime}$ and $e^{\prime \prime}$ have only one term.

\section{D.4.2. Application to hydrogen $2 \mathrm{~S}-4 \mathrm{P}$}

We now assume that the initial state $|i\rangle$ is prepared to be the $2 \mathrm{~S}_{1 / 2}^{F=0}$ state by laser excitation from the 1s ground state. Further we assume the laser, that drives the 2S-4P transition to be linearly polarized along $\left(\theta_{L}, \varphi_{L}=0\right)$ and hence use Eq. (D.5 - D.8) in Eq. (2.9). Since we are only interested in relative intensities, the reduced matrix elements in Eq. (D.5) are neglected. There are only two allowed transitions to the $4 \mathrm{P}$ excited states with $F_{e}=1, M_{e}=0, J_{e}=1 / 2, L_{e}=1$ and $F_{e}=1, M_{e}=$ $0, J_{e}=3 / 2, L_{e}=1$ whose frequency splitting is $\Delta=2 \pi \times 1367433.3(3) \mathrm{kHz} \mathrm{kHz}$ (see Fig. 2.4). The inverse life times of both of these excited states are given by $\Gamma_{e}=12.943 \mathrm{MHz}$. The detection is assumed to take place in the direction $(\theta, \varphi)$ 
via the $4 \mathrm{P} \rightarrow 1 \mathrm{~S}$ decay such that $J_{f}=1 / 2, L_{f}=0$ and $F_{f}=1,0$ and their Zeeman sublevels are possible final states. Performing the sums above yields the $2 \mathrm{~S}-4 \mathrm{P}$ explicit full line shape:

$$
\begin{aligned}
I\left(\theta, \varphi, \theta_{L}\right) \propto & \frac{1}{2}\left|\frac{1}{\omega_{L}+i \Gamma_{e} / 2}-\frac{1}{\omega_{L}-\Delta+i \Gamma_{e} / 2}\right|^{2} \\
\times & \left(\left(1+\cos (\theta)^{2}\right) \cos \left(\theta_{L}\right)^{2}+2 \cos (\theta) \cos \left(\theta_{L}\right) \cos (\varphi) \sin (\theta) \sin \left(\theta_{L}\right)\right. \\
& \left.\quad+\sin \left(\theta_{L}\right)^{2}\left(\cos (\varphi)^{2}+\sin (\theta)^{2}+\cos (\theta)^{2} \sin (\varphi)^{2}\right)\right) \\
+ & \frac{1}{2}\left|\frac{1}{\omega_{L}+i \Gamma_{e} / 2}+\frac{2}{\omega_{L}-\Delta+i \Gamma_{e} / 2}\right|^{2} \\
\times & \left(\left(\cos \left(\theta_{L}\right) \sin (\theta)-\cos (\theta) \cos (\varphi) \sin \left(\theta_{L}\right)\right)^{2}+\sin \left(\theta_{L}\right)^{2} \sin (\varphi)^{2}\right)
\end{aligned}
$$

where we set the unperturbed $2 \mathrm{~S}-4 \mathrm{P} J_{e}=1 / 2$ resonance to $\omega_{L}=0$. From Eq. (D.15) it is seen that this expression does not depend on $\varphi$. A more complicated expression for non vanishing $\varphi_{L}$ could be given, but does not bring anything new because the coordinate system can always be chosen such that $\varphi_{L}=0$. For linear laser polarization one could pick the $z$-axis to align along the laser polarization. In that case $\left(\theta_{L}=0, \varphi_{L}=0\right)$ and the full line shape model Eq. (D.15) may be used with $\eta=0$. Alternatively we can simply set $\theta_{L}=0$ in Eq. (D.24) to obtain:

$$
\begin{aligned}
I_{p=0}(\theta) \propto & \left|\frac{1}{\omega_{L}+i \Gamma_{e} / 2}-\frac{1}{\omega_{L}-\Delta+i \Gamma_{e} / 2}\right|^{2}\left(1+\cos (\theta)^{2}\right) \\
& +\left|\frac{1}{\omega_{L}+i \Gamma_{e} / 2}+\frac{2}{\omega_{L}-\Delta+i \Gamma_{e} / 2}\right|^{2} \sin (\theta)^{2}
\end{aligned}
$$

The expanded line shape of the 2S-4P transition in Sec. 2.3.2 can be either determined from Eq. (D.22-D.23) or from Eq. (D.24).

\section{D.4.3. Finite detection angle}

To obtain the fluorescence collected using a rotationally symmetric detector with an opening angle of $\theta_{c}$ centered about the $z$-axis, Eq. (D.24) is integrated:

$$
\begin{aligned}
& \int_{0}^{2 \pi} \int_{0}^{\theta_{c}} I\left(\theta, \varphi, \theta_{L}\right) \sin (\theta) d \theta d \varphi \\
& \quad \propto \frac{\pi}{24}\left|\frac{1}{\omega_{L}+i \Gamma_{e} / 2}-\frac{1}{\omega_{L}-\Delta+i \Gamma_{e} / 2}\right|^{2} \\
& \quad \times\left(64-\cos \left(3 \theta_{c}\right)-3 \cos \left(\theta_{c}\right)\left(21-4 \cos \left(2 \theta_{L}\right) \sin \left(\theta_{c}\right)^{2}\right)\right) \\
& \quad+\frac{\pi}{3}\left|\frac{1}{\omega_{L}+i \Gamma_{e} / 2}+\frac{2}{\omega_{L}-\Delta+i \Gamma_{e} / 2}\right|^{2} \\
& \quad \times\left(\left(16+8 \cos \left(\theta_{c}\right)\right) \cos \left(\theta_{L}\right)^{2} \sin \left(\frac{\theta_{c}}{2}\right)^{4}+\left(4-3 \cos \left(\theta_{c}\right)-\cos \left(\theta_{c}\right)^{3}\right) \sin \left(\theta_{L}\right)^{2}\right)
\end{aligned}
$$


For the limiting case of $\theta_{L}=\pi$, this simplifies to:

$$
\begin{aligned}
& =\frac{16 \pi}{3}\left|\frac{1}{\omega_{L}+i \Gamma_{e} / 2}-\frac{1}{\omega_{L}-\Delta+i \Gamma_{e} / 2}\right|^{2}+\frac{8 \pi}{3}\left|\frac{1}{\omega_{L}+i \Gamma_{e} / 2}+\frac{2}{\omega_{L}-\Delta+i \Gamma_{e} / 2}\right|^{2} \\
& =\frac{40 \pi}{3} \frac{1}{\omega_{L}^{2}+\left(\Gamma_{e} / 2\right)^{2}}+\frac{48 \pi}{3} \frac{1}{\left(\omega_{L}-\Delta\right)^{2}+\left(\Gamma_{e} / 2\right)^{2}}
\end{aligned}
$$

The expression above confirms that the line shape modifications due to quantum interference vanish (independent of the laser polarization) as shown in general in Sec. D.3. Note that the much smaller coherent line pulling due to one resonance sitting on the far-reaching wing of the other is still present.

The experimental implementation (Sec. 3) for spectroscopy of the 2S-4P transition uses a split detector. Using Eq. (D.24) and $\varphi=-\pi / 2+k \pi \ldots+\pi / 2+k \pi$ with $k=0$ for the $x>0$ half space and $k=1$ for the $x<0$ half space, the signals may be computed in the same way:

$$
\begin{aligned}
& \int_{-\pi / 2+k \pi}^{+\pi / 2+k \pi} \int_{0}^{\theta_{c}} I\left(\theta, \varphi, \theta_{L}\right) \sin (\theta) d \theta d \varphi \\
& \propto \\
& \propto \frac{\pi}{48}\left|\frac{1}{\omega_{L}+i \Gamma_{e} / 2}-\frac{1}{\omega_{L}-\Delta+i \Gamma_{e} / 2}\right|^{2} \\
& \times\left(64-\cos \left(3 \theta_{c}\right)-3 \cos \left(\theta_{c}\right)\left(21-4 \cos \left(2 \theta_{L}\right) \sin \left(\theta_{c}\right)^{2}\right) \pm 32\left(\sin \left(\theta_{c}\right)^{3} \sin \left(2 \theta_{L}\right)\right)\right. \\
& +\frac{\pi}{6}\left|\frac{1}{\omega_{L}+i \Gamma_{e} / 2}+\frac{2}{\omega_{L}-\Delta+i \Gamma_{e} / 2}\right|^{2} \\
& \times\left[\left(16+8 \cos \left(\theta_{c}\right)\right) \cos \left(\theta_{L}\right)^{2} \sin \left(\frac{\theta_{c}}{2}\right)^{4}+\left(4-3 \cos \left(\theta_{c}\right)-\cos \left(\theta_{c}\right)^{3}\right) \sin \left(\theta_{L}\right)^{2}\right. \\
& \left.\quad \mp 8 \sin \left(\theta_{c}\right)^{3} \sin \left(2 \theta_{L}\right)\right]
\end{aligned}
$$

In comparison to Eq. (D.27) there is a global factor of $1 / 2$ and two extra terms whose sign changes upon switching the half space (upper sign for $x>0$ and lower sign for $x<0$ ). These terms are responsible for the increased sensitivity to quantum interference line shifts using the split detector. Adding the equations for the two half spaces, the terms cancel and Eq. (D.27) is recovered.

\section{D.4.4. General Detection Geometry}

Inspecting Eq. (D.24) it becomes clear that for any detection geometry the full line shape can be written as

$$
I \propto\left|\frac{1}{\omega_{L}+i \Gamma_{e} / 2}-\frac{1}{\omega_{L}-\Delta+i \Gamma_{e} / 2}\right|^{2} A+\left|\frac{1}{\omega_{L}+i \Gamma_{e} / 2}+\frac{2}{\omega_{L}-\Delta+i \Gamma_{e} / 2}\right|^{2} B
$$


with geometry dependent functions $A$ and $B$. Examples are given in Eq. (D.25), Eq. (D.27), Eq. (D.27) and Eq. (D.29). Using the expansion of the line shape, this becomes:

$$
I \propto \frac{A+B}{\omega_{L}^{2}+\left(\Gamma_{e} / 2\right)^{2}}+\frac{(2 A+8 B) \omega_{L}}{\Delta^{3}}+\frac{(2 A-4 B) \omega_{L}}{\omega_{L}^{2}+\left(\Gamma_{e} / 2\right)^{2}}
$$

for the $\mathrm{P}_{1 / 2}$ component and

$$
I \propto \frac{A+4 B}{\omega_{L}^{2}+\left(\Gamma_{e} / 2\right)^{2}}-\frac{(2 A+2 B) \omega_{L}}{\Delta^{3}}-\frac{(4 A-2 B) \omega_{L}}{\omega_{L}^{2}+\left(\Gamma_{e} / 2\right)^{2}}
$$

for the $\mathrm{P}_{3 / 2}$ component. The corresponding line pullings are

$$
\Delta \omega\left(p_{1 / 2}\right)=\frac{\Gamma^{2}\left(4 B\left(\Gamma^{2}-\Delta^{2}\right)+A\left(\Gamma^{2}+2 \Delta^{2}\right)\right.}{4(A+B) \Delta^{3}} \approx \frac{2 A-4 B}{4(A+B)} \frac{\Gamma^{2}}{\Delta}
$$

For any cone- or point like $\left(\theta_{c}=0\right)$ detector the shifts of the $2 \mathrm{~S}-4 \mathrm{P}_{1 / 2}$ transition are of the form $c_{1}(1+3 \cos (2 \theta))$, where $c_{1}$ is a constant and depends on the detection opening angle $\theta_{c}$.

For the $2 \mathrm{~S}-4 \mathrm{P}_{3 / 2}$ transition, the shifts amount to:

$$
\Delta \omega\left(p_{3 / 2}\right)=-\frac{\Gamma^{2}\left(B\left(\Gamma^{2}-4 \Delta^{2}\right)+A\left(\Gamma^{2}+2 \Delta^{2}\right)\right.}{4(A+4 B) \Delta^{3}} \approx \frac{2 A-4 B}{4(A+4 B)} \frac{\Gamma^{2}}{\Delta}
$$

And again, for any cone- or point like $\left(\theta_{c}=0\right)$ detector these shifts are of the form $c_{1}\left(c_{2}+\cos (2 \theta)\right) /\left(c_{3}-2 \cos (2 \theta)\right)$, where $c_{1}, c_{2}, c_{3}$ are constants which depend on the detection opening angle $\theta_{c}$. The center of weight (4P fine structure centroid) is shifted by:

$$
\Delta \omega(\text { centroid })=\frac{3 B}{4(2 A+5 B)} \frac{\Gamma^{4}}{\Delta^{3}} \approx 0
$$

The ratio $\Gamma / \Delta$ is about $1 / 100$ in the case of the $2 \mathrm{~S}-4 \mathrm{P}$ transitions. This means the shifts due to quantum interference are efficiently canceled to $\mathcal{O}\left(10^{-6} \Gamma\right)$, i.e. significantly less than $100 \mathrm{~Hz}$, in a large variety of possible geometries. 


\section{E. Hydrogen apparatus: CAD drawings}

\section{E.1. 2014 measurement campaign}

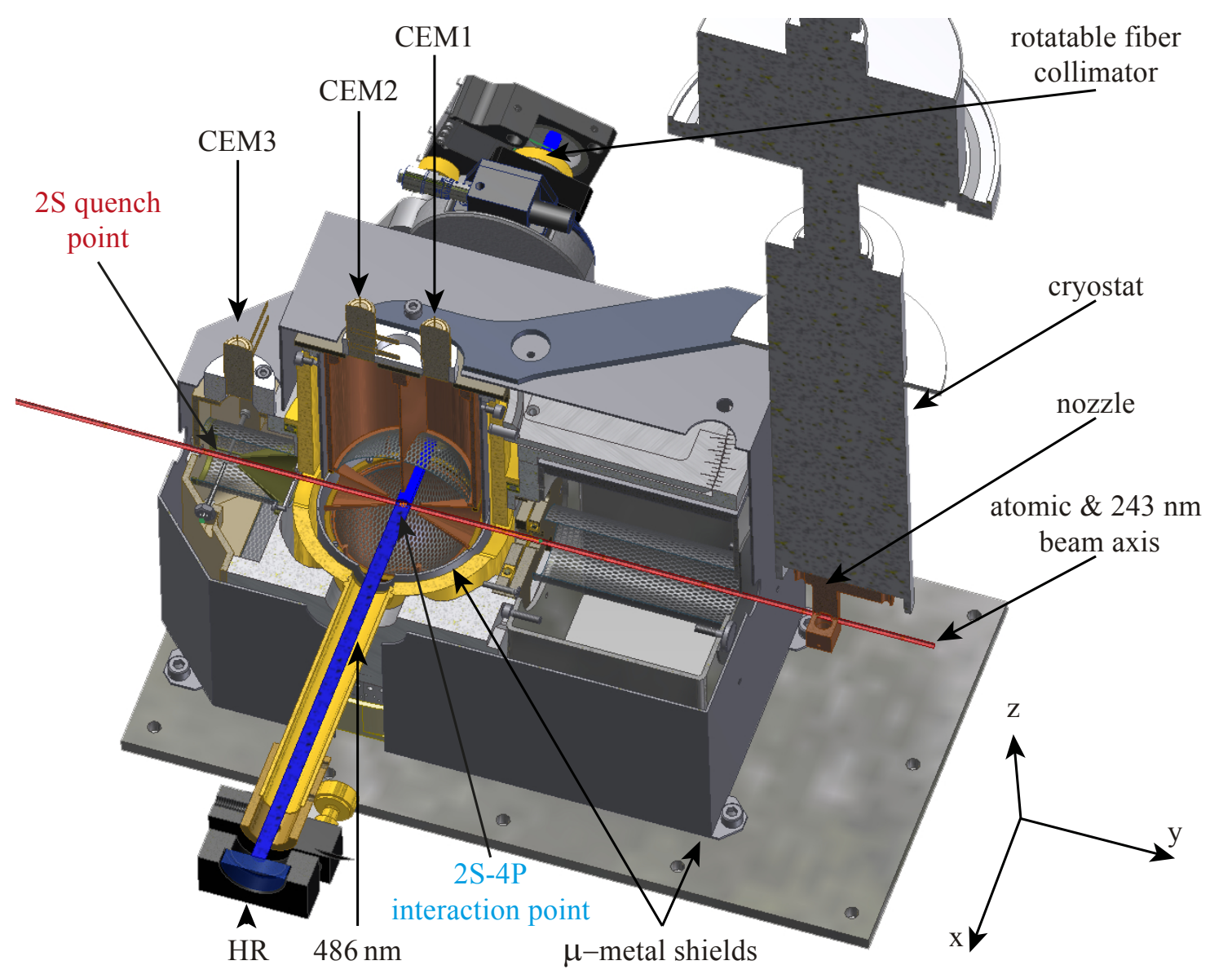

Figure E.1.: $3 \mathrm{D}$ view of cryogenic $2 \mathrm{~S}$ beam apparatus and large solid angle detector setup as discussed in Sec. 3. The AFR axis (defined by HR mirror and collimator) can be rotated with respect to the atomic beam axis. Lyman- $\gamma$ photons emitted upon the $4 \mathrm{P}-1 \mathrm{~S}$ decay are detected by channel electron multipliers (CEM 1\&2) via photo electrons emitted from the graphite coated detector walls. Due to a malfunction of the electrodes for de-excitation of the $2 \mathrm{~S}$ state, CEM3 (intended to monitor the number of $2 \mathrm{~S}$ atoms after the $2 \mathrm{~S}-4 \mathrm{P}$ interaction) has not been used for the measurements presented in the main body of this text. The coordinate system in the lower right corner serves for orientation, however the origin coincides with the $2 \mathrm{~S}-4 \mathrm{P}$ interaction point as defined before (see Fig. 2.3). 


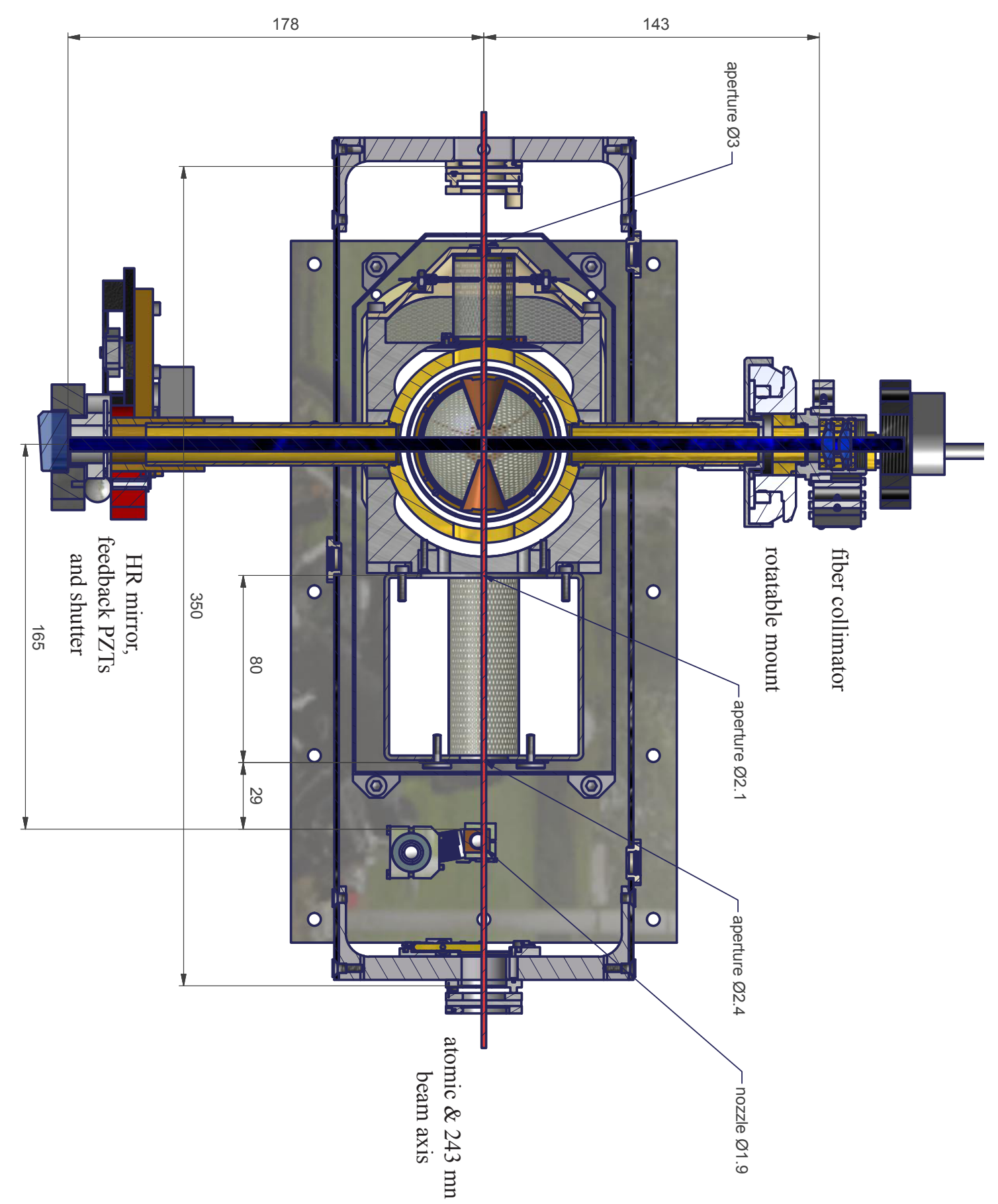

Figure E.2.: Technical drawing of cryogenic $2 \mathrm{~S}$ beam apparatus and large solid angle detector setup (cut in $\mathrm{x}$-y-plane). All distances are measured in millimeters. 


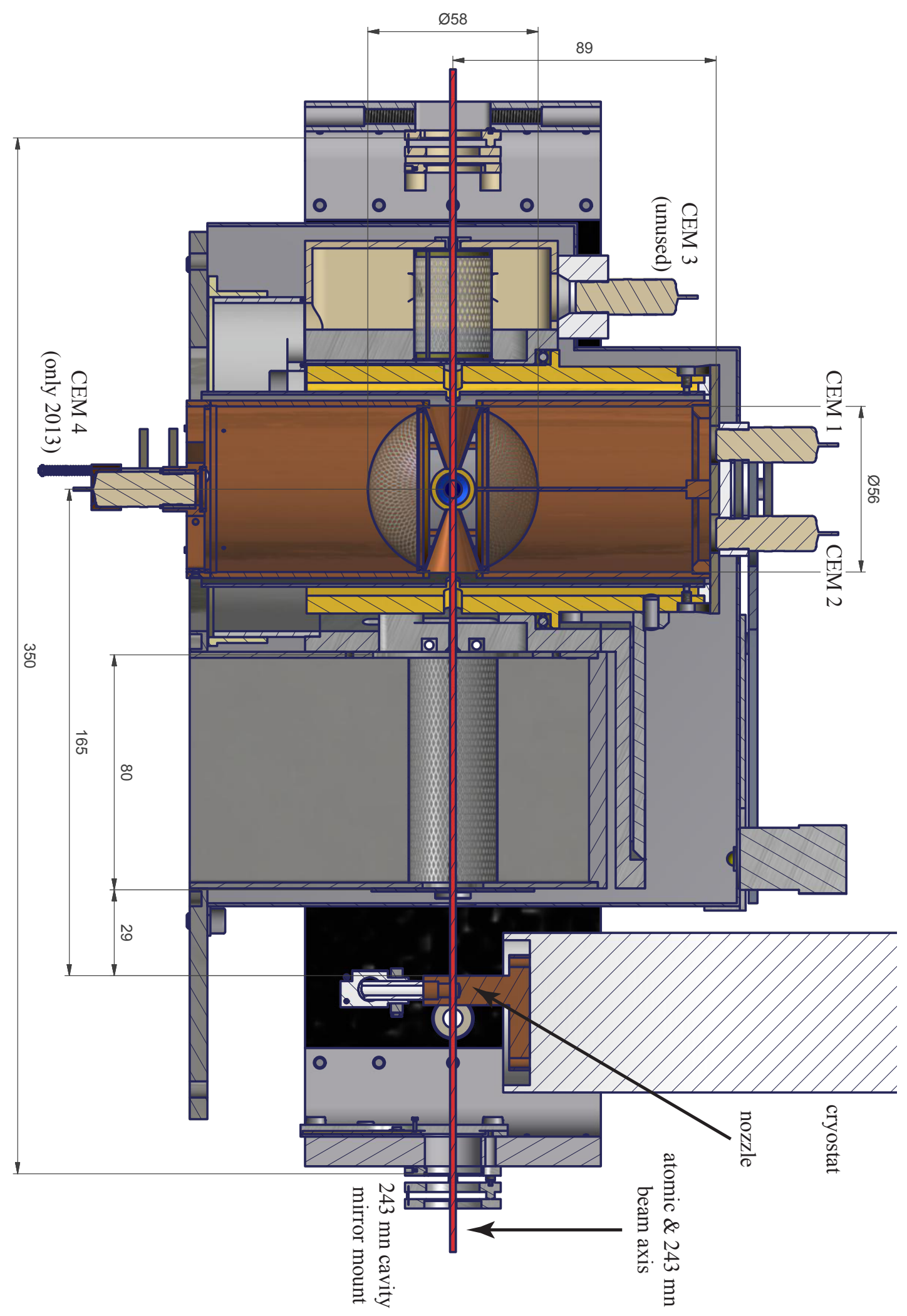

Figure E.3.: Technical drawing of cryogenic $2 \mathrm{~S}$ beam apparatus and large solid angle detector setup (cut in y-z-plain). All distances are measured in millimeters. 


\section{E.2. 2013 measurement campaign}

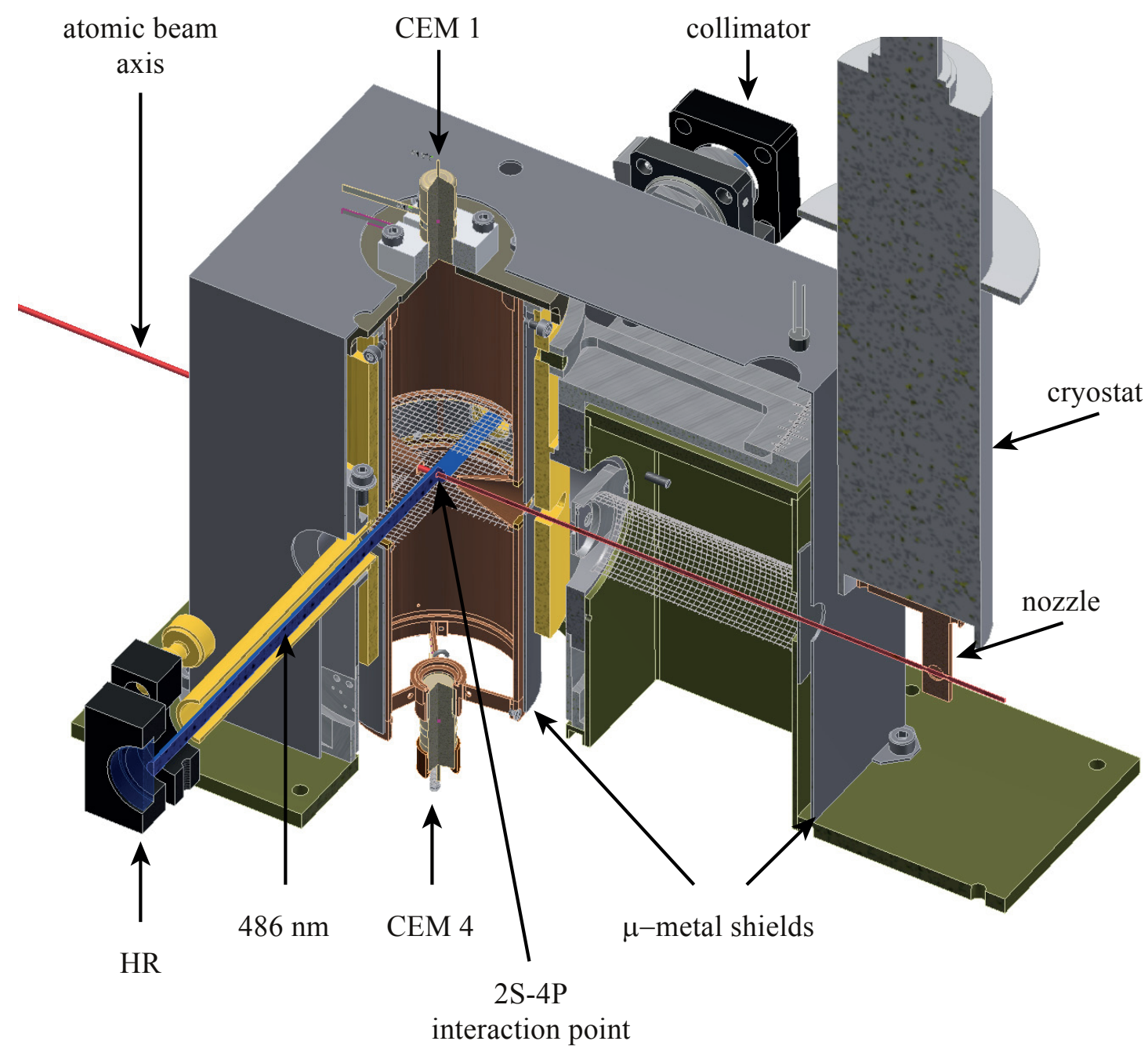

Figure E.4.: $3 \mathrm{D}$ view of cryogenic $2 \mathrm{~S}$ beam apparatus and large solid angle detector setup used in the 2013 measurement run [49]. Instead of the split detector in the 2014 measurement run, the 2013 setup features detectors above (CEM 1) and below (CEM 4) the interaction point to increase the overall solid angle coverage. 


\section{Bibliography}

[1] R. Pohl, A. Antognini, F. Nez, F. D. Amaro, F. Biraben, J. M. R. Cardoso, D. S. Covita, A. Dax, S. Dhawan, L. M. P. Fernandes, A. Giesen, T. Graf, T. W. Hänsch, P. Indelicato, L. Julien, C. Y. Kao, P. Knowles, E. O. Le Bigot, Y. W. Liu, J. A. M. Lopes, L. Ludhova, C. M. B. Monteiro, F. Mulhauser, T. Nebel, P. Rabinowitz, J. M. F. dos Santos, L. A. Schaller, K. Schuhmann, C. Schwob, D. Taqqu, J. F. C. A. Veloso, and F. Kottmann. The size of the proton. Nature, 466(7303):213-216, 2010. , 6, 9, 75

[2] A. Antognini, F. Nez, K. Schuhmann, F. D. Amaro, F. Biraben, J. M. R. Cardoso, D. S. Covita, A. Dax, S. Dhawan, M. Diepold, L. M. P. Fernandes, A. Giesen, A. L. Gouvea, T. Graf, T. W. Hänsch, P. Indelicato, L. Julien, C. Y. Kao, P. Knowles, F. Kottmann, E. O. Le Bigot, Y. W. Liu, J. A. M. Lopes, L. Ludhova, C. M. B. Monteiro, F. Mulhauser, T. Nebel, P. Rabinowitz, J. M. F. dos Santos, L. A. Schaller, C. Schwob, D. Taqqu, J. F. C. A. Veloso, J. Vogelsang, and R. Pohl. Proton Structure from the Measurement of 2S-2P Transition Frequencies of Muonic hydrogen. Science, 339(6118):417-420, 2013. , 6, 9,75

[3] R. Pohl, R. Gilman, G. A. Miller, and K. Pachucki. Muonic Hydrogen and the Proton Radius puzzle. Ann. Rev. Nuclear Particle Science, Vol 63, 63:175-204, 2013. , 6, 9, 79

[4] P. J. Mohr, B. N. Taylor, and D. B. Newell. Codata recommended values of the fundamental physical constants: 2010. Rev. Modern Phys., 84(4):1527-1605, 2012. $, 2,3,4,5,8,9,20,65,72,75,78$

[5] D. J. Berkeland, E. A. Hinds, and M. G. Boshier. Precise optical measurement of lamb shifts in atomic-hydrogen. Phys. Rev. Lett., 75(13):2470-2473, 1995. , $8,32,36,42,43,47,64$

[6] C. G. Parthey, A. Matveev, J. Alnis, B. Bernhardt, A. Beyer, R. Holzwarth, A. Maistrou, R. Pohl, K. Predehl, Th. Udem, T. Wilken, N. Kolachevsky, M. Abgrall, D. Rovera, Ch. Salomon, Ph. Laurent, and T. W. Hänsch. Improved Measurement of the Hydrogen 1S-2S Transition frequency. Phys. Rev. Lett., 107(20):203001, 2011. , 3, 4, 35, 36, 39, 42, 59, 70, 75, 78

[7] A. Matveev, C. G. Parthey, K. Predehl, J. Alnis, A. Beyer, R. Holzwarth, T. Udem, T. Wilken, N. Kolachevsky, M. Abgrall, D. Rovera, Ch. Salomon, Ph. 
Laurent, G. Grosche, O. Terra, T. Legero, H. Schnatz, S. Weyers, B. Altschul, and T. W. Hänsch. Precision Measurement of the Hydrogen 1S-2S Frequency via a 920-km Fiber link. Phys. Rev. Lett., 110(23):230801, 2013. , 3, 4, 35, 59, $70,75,78$

[8] M. Horbatsch and E. A. Hessels. Shifts from a distant neighboring resonance. Phys. Rev. A, 82(5):052519, 2010. , 8, 11, 19, 58

[9] M. Horbatsch and E. A. Hessels. Shifts from a distant neighboring resonance for a four-level atom. Phys. Rev. A, 84(3):032508, 2011. , 11, 19, 58

[10] N. Bohr. On the constitution of atoms and molecules. Philosophical Magazine, 26(6):1-25, 1913. 1

[11] A. A. Michelson and E. Morley. Fine structure of hydrogen spectrum. Philosophical Magazine, 24(46), 1887. 1

[12] P. A. M. Dirac. The quantum theory of the electron. Proceedings of the Royal Society, 117:610, 1928. 1

[13] P. A. M. Dirac. The quantum theory of the electron. part ii. Proceedings of the Royal Society, 118:351, 1928. 1

[14] W. E. Lamb and R. C. Retherford. Fine structure of the hydrogen atom by a microwave method. Phys. Rev., 72(3):241-243, 1947. 1

[15] Individual pictures taken from https://en.wikipedia.org (Emission spectrum-H.png) and blog. chungyc.org (fine-structure.png). Composition by the author of this text. 2

[16] J. J. Balmer. Notiz über die spektrallinien des wasserstoffes. Verhandlungen der Naturforschenden Gesellschaft, 7, 1885. 2

[17] F. Biraben. Spectroscopy of atomic hydrogen How is the Rydberg constant determined? European Phys. Journal-special Topics, 172:109-119, 2009. 3

[18] D. Hanneke, S. Fogwell, and G. Gabrielse. New measurement of the electron magnetic moment and the fine structure constant. Phys. Rev. Lett., 100(12):120801, 2008. 4

[19] T. Aoyama, M. Hayakawa, T. Kinoshita, and M. Nio. Tenth-order QED contribution to lepton anomalous magnetic moment: Fourth-order vertices containing sixth-order vacuum-polarization subdiagrams. Phys. Rev. D, 83(5):053002, 2011. 4

[20] S. Sturm, F. Kohler, J. Zatorski, A. Wagner, Z. Harman, G. Werth, W. Quint, C. H. Keitel, and K. Blaum. High-precision measurement of the atomic mass of the electron. Nature, 506(7489):467-470, 2014. 4 
[21] D. L. Farnham, R. S. Vandyck, and P. B. Schwinberg. Determination of the electrons atomic mass and the proton/electron mass-ratio via penning trap massspectroscopy. Phys. Rev. Lett., 75(20):3598-3601, 1995. 4

[22] A. C. Vutha and E. A. Hessels. Frequency-offset separated oscillatory fields. Phys. Rev. A, 92(5):052504, 2015. 7

[23] S. Galtier, F. Nez, L. Julien, and F. Biraben. Ultraviolet continuous-wave laser source at $205 \mathrm{~nm}$ for hydrogen spectroscopy. Optics Comm., 324:34-37, 2014. 7,78

[24] E. Peters, D. C. Yost, A. Matveev, T. W. Hänsch, and Th. Udem. Frequencycomb spectroscopy of the hydrogen 1S-3S and 1S-3D transitions. Annalen Der Physik, 525(7):L29-L34, 2013. 7, 78

[25] A. Beyer, L. Maisenbacher, A. Matveev, R. Pohl, K. Khabarova, Y. Chang, A. Grinin, T. Lamour, T. Shi, D. C. Yost, Th. Udem, T. W. Hänsch, and N. Kolachevsky. Active fiber-based retroreflector providing phase-retracing antiparallel laser beams for precision spectroscopy. Opt. Express, 24(15):1747017485, Jul 2016. 8, 35, 40, 47, 51, 64, 95

[26] B. de Beauvoir, C. Schwob, O. Acef, L. Jozefowski, L. Hilico, F. Nez, L. Julien, A. Clairon, and F. Biraben. Metrology of the hydrogen and deuterium atoms: Determination of the Rydberg constant and Lamb shifts. European Phys. J. D, 12(1):61-93, 2000. 8

[27] A. Marsman, M. Horbatsch, and E. A. Hessels. Shifts due to neighboring resonances for microwave measurements of the 2 P-3 fine structure of helium. Phys. Rev. A, 86(1):012510, 2012. 11, 19, 58

[28] A. Marsman, M. Horbatsch, and E. A. Hessels. Shifts due to distant neighboring resonances for laser measurements of 2 S-3(1)-to-2 P-3(J) transitions of helium. Phys. Rev. A, 86(4):040501, 2012.

[29] A. Marsman, E. A. Hessels, and M. Horbatsch. Shifts due to quantummechanical interference from distant neighboring resonances for saturated fluorescence spectroscopy of the $2(3) \mathrm{S}$ to $2(3) \mathrm{P}$ intervals of helium. Phys. Rev. A, 89(4):043403, 2014. 11, 58

[30] C. J. Sansonetti, C. E. Simien, J. D. Gillaspy, J. N. Tan, S. M. Brewer, R. C. Brown, S. Wu, and J. V. Porto. Absolute Transition Frequencies and Quantum Interference in a Frequency Comb Based Measurement of the Li-6,Li-7 D lines. Phys. Rev. Lett., 107(2):023001, 2011. 11

[31] R. C. Brown, S. Wu, J. V. Porto, C. J. Sansonetti, C. E. Simien, S. M. Brewer, J. N. Tan, and J. D. Gillaspy. Quantum interference and light polarization 
effects in unresolvable atomic lines: Application to a precise measurement of the Li-6,Li-7 D-2 lines. Phys. Rev. A, 87(3):032504, 2013. 11, 15, 19, 28, 42, 43

[32] D. J. Griffiths. Introduction to electrodynamics. Prentice-Hall, Inc., New Jersey, 1999. 12

[33] U. D. Jentschura and P. J. Mohr. Nonresonant effects in one- and two-photon transitions. Canadian J. Phys., 80(6):633-644, 2002. 13, 21, 108

[34] Z. Ficek and S. Swain. Quantum Interference and Coherence. Springer Series in Optical Sciences, 2005. 15, 18

[35] S. Schippers. Analytic expression for the convolution of a fano line profile with a gaussian. Int. Rev. At. Mol. Phys., 2(151), 2012. 15

[36] R. Loudon. The Quantum Theory of Light. Oxford University Press, 2000. 16

[37] I. I. Sobelman. Atomic Spectra and Radiative Transitions. Springer Verlag, Berlin-Heidelberg New York, 1979. 16, 103, 104

[38] G. S. Agarwal. Quantum Statistical Theories of Spontaneous Emission and Their Relation to Other Approaches, , edited by G. Möhler et al., Springer Tracts in Modern Physics: Quantum Optics Vol. 70. Springer-Verlag, Berlin, 1974. 18

[39] D. A. Cardimona, M. G. Raymer, and C. R. Stroud. Steady-state quantum interference in resonance fluorescence. J. Phys. B-atomic Mol. Opt. Phys., 15(1):55-64, 1982. 19

[40] D. A. Cardimona and C. R. Stroud. Spontaneous radiative coupling of atomicenergy levels. Phys. Rev. A, 27(5):2456-2461, 1983. 19

[41] D. C. Yost, A. Matveev, E. Peters, A. Beyer, T. W. Hänsch, and Th. Udem. Quantum interference in two-photon frequency-comb spectroscopy. Phys. Rev. A, 90(1):012512, 2014. 19, 25, 32, 58

[42] S. Haroche. Topics in Applied Physics, Vol. 13, Chap. 7, pp. 253-313. Springer Verlag, edited by K. Shimoda, Berlin, 1976. 19

[43] Physical Reference Data according to "CODATA Recommended Values of the Fundamental Physical Constants:2010". Web page of the Physical Measurement Laboratory, National Institute of Standards and Technology, Gaithersburg, USA: http://physics.nist.gov/PhysRefData/HDEL/transfreq.html. 20,65

[44] A. E. Kramida. A critical compilation of experimental data on spectral lines and energy levels of hydrogen, deuterium, and tritium. Atomic Data Nuclear Data Tables, 96(6):586-644, 2010. 20 
[45] M. Horbatsch and E. A. Hessels. Tabulation of the bound-state energies of atomic hydrogen. Phys. Rev. A, 93(2):2513-2513, 2016. 20, 65, 73, 74

[46] N. Kolachevsky, J. Alnis, C. G. Parthey, A. Matveev, R. Landig, and T. W. Hänsch. Low phase noise diode laser oscillator for 1S-2S spectroscopy in atomic hydrogen. Optics Lett., 36(21):4299-4301, 2011. 35, 39, 72

[47] C.G. Parthey. Precision Spectroscopy on atomic hydrogen. PhD thesis, LudwigMaximilians-Universität München, 2011. 35, 36, 39, 59, 70

[48] J. Alnis, A. Matveev, N. Kolachevsky, Th. Udem, and T. W. Hänsch. Subhertz linewidth diode lasers by stabilization to vibrationally and thermally compensated ultralow-expansion glass Fabry-Perot cavities. Phys. Rev. A, 77(5):053809, 2008. 39, 72

[49] A. Beyer, J. Alnis, K. Khabarova, A. Matveev, C. G. Parthey, D. C. Yost, R. Pohl, Th. Udem, T. W. Hänsch, and N. Kolachevsky. Precision spectroscopy of the $2 \mathrm{~S}-4 \mathrm{P}$ transition in atomic hydrogen on a cryogenic beam of optically excited 2S atoms. Annalen Der Physik, 525(8-9):671-679, 2013. 41, 44, 55

[50] W. Demtröder. Laser Spectroscopy (Vol.2). Springer-Verlag, Berlin Heidelberg, 2008. 42

[51] See for instance HM-15-05 from PLX Inc., NY. Reflectivity per surface: $96 \%$ $\rightarrow 88 \%$ total reflectivity. 42,98

[52] A. Beyer, L. Maisenbacher, K. Khabarova, A. Matveev, R. Pohl, Th. Udem, T. W. Hänsch, and N. Kolachevsky. Precision spectroscopy of 2S-nP transitions in atomic hydrogen for a new determination of the Rydberg constant and the proton charge radius. Phys. Scr., T165:014030, 2015. 1, 43, 44

[53] W. He, Y. Fu, Y. Zheng, L. Zhang, J. Wang, Z. Liu, and J. Zheng. Polarization properties of a corner-cube retroreflector with three-dimensional polarization ray-tracing calculus. Appl. Optics, 52(19):4527-4535, 2013. 43

[54] H. Müller, S. W. Chiow, Q. Long, C. Vo, and S. Chu. Active sub-Rayleigh alignment of parallel or antiparallel laser beams. Optics Lett., 30(24):33233325, 2005. 43, 101

[55] F. Minardi, M. Artoni, P. Cancio, M. Inguscio, G. Giusfredi, and I. Carusotto. Frequency shift in saturation spectroscopy induced by mechanical effects of light. Phys. Rev. A, 60(5):4164-4167, 1999. 43, 51

[56] S. Hannemann, E. J. Salumbides, and W. Ubachs. Reducing the first-order doppler shift in a sagnac interferometer. Opt. Lett., 32(11):1381-1383, Jun 2007. 43 
[57] A. Beyer, C. G. Parthey, N. Kolachevsky, J. Alnis, K. Khabarova, R. Pohl, E. Peters, D. C. Yost, A. Matveev, K. Predehl, S. Droste, T. Wilken, R. Holzwarth, T. W. Hänsch, M. Abgrall, D. Rovera, Ch. Salomon, Ph. Laurent, and Th. Udem. Precision Spectroscopy of Atomic hydrogen. 21st Int. Conference On Laser Spectr. - Icols 2013, 467:012003, 2013. 44

[58] Nufern S405 XP polarization maintaining fiber, mode field diameter MFD = $3.8(5) \mu \mathrm{m}\left(1 / e^{2}\right.$ intensity diameter, interpolated at $\left.486 \mathrm{~nm}\right), w_{o}=1.9(25) \mu \mathrm{m}$ (1/e field radius). AR coated by Diamond SA Via dei Patrizi 5, CH-6616 Losone. $R \leq 0.1 \%$. 44, 45, 101

[59] Combination of two cemented achromatic lens doublets from QIoptiq, G3222100000 $(f=80 \mathrm{~mm})$ and G32229000 $(f=40 \mathrm{~mm})$ equipped with AR coating $(R \leq 0.1 \%)$. 44, 96, 97, 98, 99, 101

[60] E. G. Neumann. Single Mode Fibers: Fundamentals. Springer-Verlag, Berlin, 1988. 44

[61] Dielectric high reflecting miror. $R=99.995 \%$ at $486 \mathrm{~nm}$. Custom made by Advanced Thin Films Boulder, CO. 44, 98

[62] J. Dupont-Roc C. Cohen-Tannoudji and G. Grynberg. Atom - Photon Interactions: Basic Process and Appilcations. Wiley-VCH Verlag GmbH, Weinheim, 2004. 51

[63] R. Grimm and J. Mlynek. The effect of resonant light pressure in saturation spectroscopy. Appl. Phys. B-photophysics Laser Chem., 49(3):179-189, 1989. 66

[64] D. J. Berkeland. A Precise Measurement of Lamb Shifts in Hydrogen. PhD thesis, Yale University, New Haven, 1995. 70

[65] N. Kolachevsky, A. Matveev, J. Alnis, C. G. Parthey, S. G. Karshenboim, and T. W. Hänsch. Measurement of the 2S Hyperfine Interval in Atomic hydrogen. Phys. Rev. Lett., 102(21):213002, 2009. 73

[66] C. G. Parthey, A. Matveev, J. Alnis, R. Pohl, Th. Udem, U. D. Jentschura, N. Kolachevsky, and T. W. Hänsch. Precision Measurement of the HydrogenDeuterium 1S-2S Isotope shift. Phys. Rev. Lett., 104(23):233001, 2010. 78

[67] J. Friese, R. Gernhauser, J. Homolka, A. Kastenmuller, P. Maier-Komor, M. Peter, K. Zeitelhack, P. Kienle, and H. J. Korner. Enhanced quantum efficiency for CsI grown on a graphite-based substrate coating. Nuclear Instruments \&6 Methods In Phys. Research Section A - Accelerators Spectrometers Detectors Associated Equipment, 438(1):86-93, 1999. 91

[68] J. P. Snyder. Flattening the Earth: Two Thousand Years of Map Projections. University Of Chicago Press, Chicago, 1993. 92 
[69] A. E. Siegman. Lasers. University Science Books, Sausalito, CA, 1986. 95

[70] Thorlabs SM1Z translation mount. 96

[71] Qioptiq Photonics GmbH \& Co. KG, Königsallee 23, D-37081 Göttingen, Germany, http://winlens.de/. 98

[72] Lens-Optics GmbH, Bürgermeister-Neumeyr-Strasse 7, D-85391 Allershausen, Germany. Custom made collimator $(\mathrm{f}=30 \mathrm{~mm})$ for wavelengths between $380 \mathrm{~nm}$ and $410 \mathrm{~nm} .99$

[73] Mirror mount: Radiant Dyes Laser Accessories GmbH, MDI-H-2-1". Two Newport 8301-UHV picomotor actuators have been installed in our lab. 101

[74] E. E. Bethe, H. A. and Salpeter. Quantum Mechanics of One- and Two-Electron Atoms. Dover Publications, INC., New York, 2008. 104

[75] A. Messiah. Quantum Mechanics, Vol. 2. Appendix C.I and C.II. North-Holland, Amsterdam, 1981. 104

[76] Wolfram, 2016. http://mathworld.wolfram.com/Wigner3j-Symbol.html and Wigner6j-Symbol.html. 104 



\section{Acknowledgments}

First of all I would like to thank Professor Theodor W. Hänsch for the opportunity to contribute to a project as unique as the hydrogen experiment in Garching. Your open and cordial nature, your seemingly endless treasure trove of advice concerning this experiment, your great support and the large amount of freedom and personal responsibility you provided me with in managing the experiment and representing our research group made the last five years an invaluable experience for me.

I would like to thank Professor Hänsch and Thomas Udem for the lectures, seminars and presentations that got me interested in precision spectroscopy as a master student. It was a great pleasure for me to work with you and Nikolai Kolachevsy, Christian Parthey, Randolf Pohl, Arthur Matveev and Janis Alnis during my master thesis. Thanks to all of you I decided to stay at MPQ as a PhD student and continue the efforts towards a new measurement of the Rydberg constant that we started together during my master thesis. Thomas, thank you for always having a sympathetic ear for my questions and problems over the years and for never giving up explaining until you had the impression, that I really understood.

I would like to thank the members of the hydrogen team - Ksenia Khabarova, Lothar Maisenbacher, Arthur, Nikolai, Randolf, Dylan Yost, Thomas and Professor Hänsch - for their support. The results presented in this thesis are the product of a successful team work and would not have been possible without you.

Nikolai and Ksenia have been my companions for the lion part of the hot phases in the lab, especially during the development phase of the experiment. During the long hours of testing and improving, we became friends and spend great times inside and outside the lab. Whenever unexpected challenges showed up, I could count on your support. Taming the complex hydrogen apparatus is not an easy task and Nikolai provided me with the experimental knowledge necessary to let me get the most out of the project. Last but not least, I would like to thank both of you for an unforgettable trip to Russia!

Randolf, above the many ideas and the advice you contributed to the experiment, the passion you brought into the project was a vital ingredient for its success. Thank you for sharing this passion with me and for the many interesting but also for the serious conversations and discussions we had about the experiment, science in general and topics far beyond that.

Arthur, with your success in managing the complex simulations of the quantum interference effect for the $2 \mathrm{~S}-4 \mathrm{P}$ and $1 \mathrm{~S}-3 \mathrm{~S}$ experiments, you have made yourself the champion of numerical simulations in our group. The way you dealt with those thousands of differential equations - almost making it seem like an easy task repeatedly impressed me. 
Dylan and Tobi Lamour, thank you for sharing your knowledge about feedback loops and for your help in taming some of the most refractory components in the experiment. The upgrades we implemented together have been among the key factors for the success of the last measurement campaign, especially after Murphy's law forced me to face the data taking in "lone worrier mode".

Lothar is the new $\mathrm{PhD}$ student working on the hydrogen experiment and took over the lab after I completed the data acquisition for the $2 \mathrm{~S}-4 \mathrm{P}$ project. Lothar, I wish you every success with the upcoming measurements and I am sure that your style of working will have significant impact on the hydrogen lab.

I would like to thank Randolf and the members of his team at MPQ - Marc Diepold, Beatrice Franke and Julian Krauth - as well as Masaki Hori, Christoph Gohle and Savely Karschenboim for numerous crash courses and discussions all around physics, which helped my fundamental understanding and for the preparation of various talks and presentations.

The high visibility of the hydrogen experiment at MPQ and Professor Hänsch's generous travel policy granted me with the opportunity to present our work at various international conferences all around the world. I would like to thank Professor Hänsch for the colorful experiences and impressions I gathered during these trips and the colleagues I met there - and in particular Eric Hessels, Aldo Antognini, Paolo Crivelli, Guy Ron, Franz Kottmann and Francois Nez - for the stimulating discussions and enjoyable times we shared.

I would like to thank our technicians Wolfgang Simon and Karl 'Charlie' Linner for their patience, persistence and unbelievable ingenuity which (not always willingly) I put to the test by ever more extravagant ideas and requirements, especially during the development phase of the experiment. Working with you will be among the things I am going to miss most when I leave MPQ!

Moreover, I would like to thank Helmut Brückner who provides many electronics for our entire research group and I would like to express my sincere appreciation for the administrative help of Professor Hänsch's secretaries, Ingrid Hermann and Gabriele Gschwendtner.

I would like to thank Professor Hänsch's entire research group as well as my colleagues and friends from the other divisions at MPQ for the enjoyable atmosphere. I had a lot of fun with you during activities such as the MPQ PhD summer symposium, the $\mathrm{PhD}$ Student Condensate, going out in Munich or the numerous biking and hiking trips to mention but a few. But also when I got stuck with a special or unusual problem in the lab, somebody was always there to help me out.

Last but not least I would like to thank my family and friends. You had my back, when I needed your support, and you took my mind of things whenever this was possible (or necessary). Thank you for the uncountable number of small surprises such as care-packages, breakfast bags at my door or nightly coffee deliveries - which sweetened my days (or nights) especially during hot phases of the experiment.

To cut a long story short: You are just the best! $:$; 


\section{List of publications and presentations}

\section{Manuscripts in preparation}

By the time of submission of this thesis, the following manuscripts presenting results from this work are in preparation:

1. A. Beyer et al. The Rydberg constant and proton size from atomic hydrogen. In preparation.

2. A. Beyer et al. Shifts due to quantum-mechanical interference from distant neighboring resonances for precision spectroscopy of the $2 \mathrm{~S}-4 \mathrm{P}$ transition in atomic hydrogen. Working title, in preparation.

\section{List of publications}

1. A. Beyer, L. Maisenbacher, A. Matveev, R. Pohl, A. Grinin, K. Khabarova, Y. Chang, T. Lamour, T. Shi, D.C. Yost, Th. Udem, T. W. Hänsch,

N. Kolachevsky: An active fiber-based retroreflector providing phase-retracing anti-parallel laser beams for precision spectroscopy. Optics Express 07/2016; 24, 017470 .

2. R. Pohl, F. Nez, Th. Udem, A. Antognini, A. Beyer, H. Fleurbaey, A. Grinin, T. W. Hänsch, L. Julien, F. Kottmann, J. J. Krauth, L. Maisenbacher,

A. Matveev, F. Biraben: Deuteron charge radius from spectroscopy data in atomic deuterium. arXiv 07/2016; 1607.03165.

3. A. Beyer, L. Maisenbacher, K. Khabarova, A. Matveev, R. Pohl, Th. Udem, T. W. Hänsch, N. Kolachevsky: Precision spectroscopy of $2 \mathrm{~S}-n P$ transitions in atomic hydrogen for a new determination of the Rydberg constant and the proton charge radius. Physica Scripta 10/2015; 2015(165-T165):014030.

4. D. C. Yost, A. Matveev, E. Peters, A. Beyer, T. W. Hänsch, Th. Udem: Quantum interference in two-photon frequency-comb spectroscopy. Physical Review A $07 / 2014 ; 90(1-1)$. 
5. A. Beyer, C. G. Parthey, N. Kolachevsky, J. Alnis, K. Khabarova, R. Pohl, E. Peters, D. C. Yost, A. Matveev, K. Predehl, S. Droste, T. Wilken, R. Holzwarth, T. W. Hänsch, M. Abgrall, D. Rovera, C. Salomon, P. Laurent, Th. Udem: Precision Spectroscopy of Atomic Hydrogen. Journal of Physics Conference Series 12/2013.

6. A. Beyer, J. Alnis, K. Khabarova, A. Matveev, C. G. Parthey, D. C. Yost, R. Pohl, Th. Udem, T. W. Hänsch, N. Kolachevsky: Precision spectroscopy of the $2 \mathrm{~S}-4 \mathrm{P}$ transition in atomic hydrogen on a cryogenic beam of optically excited 2S atoms. Annalen der Physik 09/2013; 525(8-9):671.

7. A. Matveev, C. G. Parthey, K. Predehl, J. Alnis, A. Beyer, R. Holzwarth, Th. Udem, T. Wilken, N. Kolachevsky, M. Abgrall, D. Rovera, C. Salomon, P. Laurent, G. Grosche, O. Terra, T. Legero, H. Schnatz, S. Weyers, B. Altschul, T. W. Hänsch: Precision Measurement of the Hydrogen 1S-2S Frequency via a 920-km Fiber Link. Physical Review Letters 06/2013; 110(23).

8. C. G. Parthey, A. Matveev, J. Alnis, B. Bernhardt, A. Beyer, R. Holzwarth, A. Maistrou, R. Pohl, K. Predehl, Th. Udem, T. Wilken, N. Kolachevsky, M. Abgrall, D. Rovera, C. Salomon, P. Laurent, T. W Hänsch: Improved Measurement of the Hydrogen 1S - 2S Transition Frequency. Physical Review Letters 11/2011; 107(20):203001. 


\section{Invited talks}

Hefei, CN (2015)

International Workshop on e+ e- collisions from Phi to Psi 2015, University of Science and Technology of China

Moscow, RU (2015)

International conference on quantum technology, Russian Quantum Center

Mendeleevo, RU (2015) Colloquium at the National Research Institute of Physical, Technical and Radiotechnical

Measurements

Rehovot, IL (2015)

Colloquium at the Weizmann Institute of Science

Jerusalem, IL (2015)

Colloquium at the Racah Institute of Physics, Hebrew University of Jerusalem

Sofia, BG (2014)

$23^{\text {rd }}$ Annual International Laser Physics Workshop, Institute of Electronics,

Bulgarian Academy of Science

Waldhausen, DE (2014) Workshop "Proton Radius Puzzle", Mainz Institute for Theoretical Physics

Garching, DE (2013) Colloquium at the

Max Planck Institute of Quantum Optics

Prague, CZ (2013)

Frontiers of Quantum and Mesoscopic Thermodynamics, Institute of Physics \& the Academy of Sciences of the Czech Republic

Trento, IT (2012)

ECT* Workshop on the "Proton Radius Puzzle", European Center for Theoretical Studies in Nuclear Physics and related areas

Zurich, CH (2012)

International workshop on Zeeman deceleration, Swiss Federal Institute of Technology (2012) 


\section{Contributed Presentations}

Eltville a. R., DE (2015)

Castle Ringberg, DE

(2011 - 2015)

Washington D.C., US (2014)

Rio de Janeiro, BR (2014)

Hannover, DE (2013)

San Francisco, US (2013)

Paris, FR (2012)

Eltville a. R., DE (2012)

Stuttgart, DE (2012)

Dresden, DE (2011)
Fundamental constants meeting 2015

Annual Symposium of the Laser Spectroscopy Division at the MPI of Quantum Optics (one talk per year)

International Conference on Atomic Physics

International Conference on Precision Physics of Simple Atomic Systems

Spring congress of the German physics society International Conference on Laser Spectroscopy International Conference on Atomic Physics International Conference on Precision Physics of Simple Atomic Systems

Spring congress of the German physics society Spring congress of the German physics society 